

\section{DISCLAIMER}

This report was prepared as an account of work sponsored by an agency of the United States Government. Neither the United States Government nor any agency Thereof, nor any of their employees, makes any warranty, express or implied, or assumes any legal liability or responsibility for the accuracy, completeness, or usefulness of any information, apparatus, product, or process disclosed, or represents that its use would not infringe privately owned rights. Reference herein to any specific commercial product, process, or service by trade name, trademark, manufacturer, or otherwise does not necessarily constitute or imply its endorsement, recommendation, or favoring by the United States Government or any agency thereof. The views and opinions of authors expressed herein do not necessarily state or reflect those of the United States Government or any agency thereof. 


\section{DISCLAIMER}

Portions of this document may be illegible in electronic image products. Images are produced from the best available original document. 


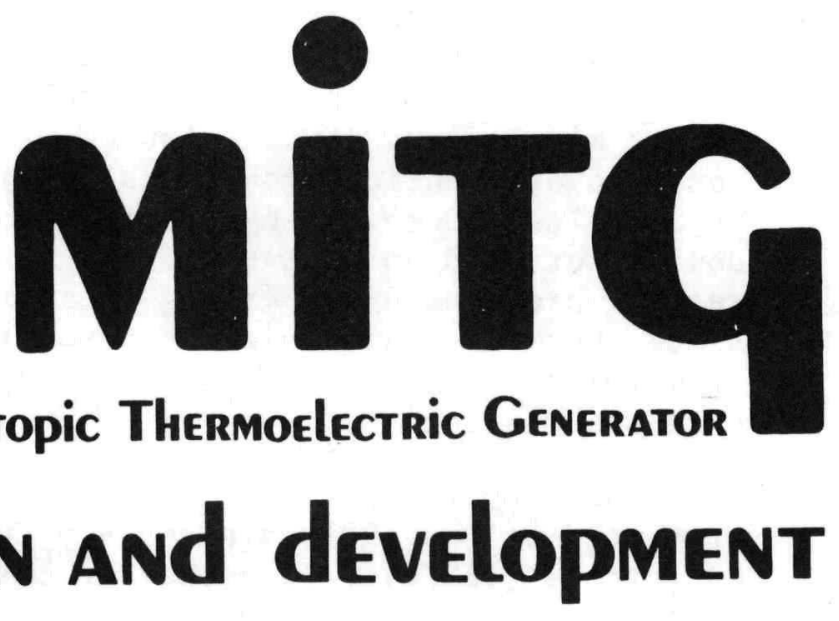




\section{INTRODUCTION AND SUMMARY}

This report contains a sequence of five papers dealing with an advanced Radioisotope Thermoelectric Generator (RTG), referred to as the Modular Isotopic Thermoelectric Generator (MITG) because of its modularity. Except for end section, the generator is composed of identical modular slices, each producing over 20 watts at 28 volts. Once the basic generator slice has been developed, a design for a 28-volt generator of any required power level can be readily produced by inserting an appropriate number of modular slices between standardized end sections. This modularity essentially eliminates the need for developing a custom-made generator for each new mission.

The report consists of five parts. Part A contains a paper presented at the 1981 Intersociety Energy Conversion Engineering Conference (IECEC). It described the design, fabrication, key features, and performance analysis of the MITG.

Because of the design's attractive features, and because its predicted power-to-weight ratio was almost double that of current RTGs, the Energy Department's Office of Special Nuclear Projects embarked on an extended program to build and test prototypical MITG slices; primarily to measure their power output and efficiency, and to demonstrate their fabricability, performance reproducibility, and long-term stability. The results of that ongoing program are described in Parts B through E, consisting of a sequence of four papers slated for presentation at the 1983 IECEC.

Part B describes the design and fabrication of the MITG test assembly. Each assembly consists of a prototypical generator slice, plus the necessary end sections. The successful fabrication of those test assemblies confirmed the feasibility of the basic converter fabrication and assembly procedure described earlier in Part A.

Part $\mathrm{C}$ describes the test plan, test methodology, instrumentation, computer-controlled test operation and data acquisition, and the initial test results. The test equipment, instrumentation, and computer system worked very well, but anomalous test results indicated that thermal-stress failures had occurred in the thermoelectric converter modules. This was confirmed by post-test examination of the affected hardware. 
Part $D$ describes detailed thermal-stress analyses to determine the cause of the observed failures, and to evaluate proposed corrective actions. Excellent agreement between the analytical results and the experimental observations lends confidence in the validity of the analyses, which clearly established the dominant cause of the observed failures. Corrective actions were identified, and analyses demonstrated that they resulted in almost complete elimination of the previous thermal-stress effects.

Finally, Part E describes additional design modifications for eliminating the observed thermal-stress effects and for improving hermeticity and thermal conductance between the thermoelectric converter modules and the generator housing. These design modifications have been validated analytically and are undergoing experimental evaluation, with very encouraging results.

Based on the above design modifications and on updated thermoelectric properties, the MITG design was reoptimized and the converter fabrication procedure was revised and improved. These changes are also described in Part E. Thermoelectric converter modules incorporating these design and fabrication changes are being fabricated, and a full set of these will be installed in new test assemblies simulating a complete MITG slice. This will permit testing of the revised units in a realistic generator environment.

As shown in Part $\mathrm{E}$, the power-to-weight ratio of the revised MITG design is about $10 \%$ lower than that predicted in 1981 , primarily because of changes in thermoelectric properties and miscellaneous electrical and thermal losses. But it is still about $78 \%$ higher than the corresponding ratio for current-generation RTGs.

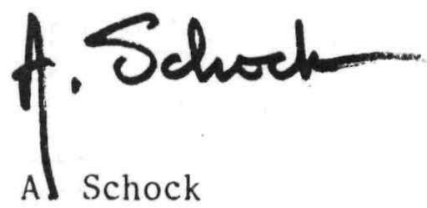

April 1983 
A. Modular Isotopic Thermoelectric Generator*

Abstract . . . . . . . . . . . . . A 1

1. Introduction .................. A 2

2. Overview of MITG Design ............. A 4

3. Thermoelectric Material Properties......... A 8

4. Thermoelectric Module Design . . . . . . . . . . . . A12

5. Thermoelectric Module Fabrication . . . . . . . . . A16

6. Thermoelectric Hot Shoe . . . . . . . . . . . . . A24

7. MITG Housing and Radiator Fins . . . . . . . . . . . A30

8. Thermal Insulation . . . . . . . . . . . . . . . . A33

9. Converter Assembly Procedure . . . . . . . . . . . A40

10. Electrical Circuit . . . . . . . . . . . . . . A47

11. Heat Source Support . . . . . . . . . . . . A53

12. Generator Assembly .. . . . . . . . . . . . A60

13. Weight Comparison ............... A66

14. Effect of Cold-Junction Temperature . . . . . . . . A66

15. Illustrative Design Summary . . . . . . . . . . . A69

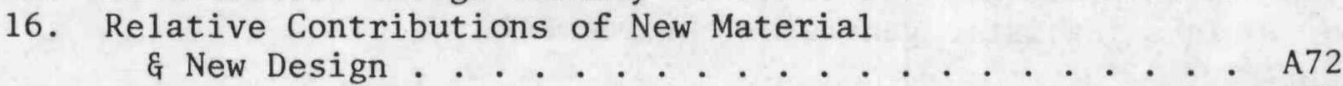

17. Conclusion ............... A73

B. MITG Test Assembly Design and Fabrication**

Abstract ................ B 2

1. Introduction ................. B 2

2. Test Objectives .............. . . B 8

3. Overview of Test Assembly Design . . . . . . . . . . B 9

4. Multicouple Design and Fabrication . . . . . . . . . B10

5. Test Housing and Insulation Design . . . . . . . . B20

6. Installation of Insulation and Multicouples . . . . . B23

7. Installation of Heater and Closure of

Test Assembly. . . . . . . . . . . . . . . . B29

8. Test Results ................ B33

C. MITG Test Procedure and Results**

Abstract ................... C 1

I. Introduction and Summary ............. C 2

II. Discussion ................. . C 8

A. Vacuum Stations .............. C10

B. Computer ................. C12

C. Software . . . . . . . . . . . . . . . C C13

D. Test Operations ............. C C30

III. Results .................. . C33 
TABLE OF CONTENTS (Cont'd.)

$\underline{\text { Page }}$

D. MITG Post-Test Analysis and Design Improvements**
Abstract.................. . D 2
1. Introduction. . . . . . . . . . . . . . . D 3
2. Test Results... . . . . . . . . . . . . . . D 4
3. Therma1-Stress Problem . . . . . . . . . . . . D 6
4. Thermal Stress Analysis . . . . . . . . . . . . . . . D 9
5. Dominant Source of Thermal Stresses . . . . . . . . . . D14
5.1 Bimetallic Cold Stud . . . . . . . . . . . . . . D15
5.2 Mismatched Expansion Coefficients . . . . . . . . D16
5.3 Effect of Temperature Gradient . . . . . . . . . . D17
5.4 Wa11 Expansion Effect . . . . . . . . . . . . . D21
6. E1imination of Thermal Stress . . . . . . . . . . D23
6.1 Compliant Cushion . . . . . . . . . . . . . . . . D23
6.2 Double-Headed Cold Stud . . . . . . . . . . . . D26
6.3 Graphite Heat Collector . . . . . . . . . . . . . . D31
6.4 Vibration Test . . . . . . . . . . . . . . D36
7. Summary . . . . . . . . . . . . . . D40

E. Revised MITG Design, Fabrication Procedure, and

Performance Predictions**

Abstract . . . . . . . . . . . . . . E 2

1. Introduction . . . . . . . . . . . . . E 3

2. Multicouple Redesign .. . . . . . . . . . . . . E 4

2.1 Double-Headed Cold Stud . . . . . . . . . . . . . . I 4

2.2 Beveled Washer at Cold Stud . . . . . . . . . E 6

2.3 Conical Stud Head . . . . . . . . . . . . . . . E10

3. Updated MITG Design . . . . . . . . . . . . . . E16

3.1 Material Properties Changes . . . . . . . . . E16

3.2 Thermopile Redesign . . . . . . . . . . . . . . . E19

3.3 Revised Fabrication Process . . . . . . . . . . . E21

3.4 Weight and Performance Summary . . . . . . . E28

*Presented at the 1981 IECEC

${ }^{*}$ *For presentation at the 1983 IECEC 
Part A:

\title{
Modular Isotopic Thermoelectric Generator
}

\author{
A. Schock \\ Fairchild Space and Electronics Company \\ Germantown, Maryland 20874
}

(Presented at the 1981 Intersociety Energy Conversion Engineering Conference)

\begin{abstract}
Advanced RTG concepts utilizing improved thermoelectric materials and converter concepts are under study at Fairchild for DOE. The design described here is based on DOE's newly developed radioisotope heat source, and on an improved silicon-germanium material and a multicouple converter module under development at Syncal. Fairchild's assignment was to combine the above into an attractive power system for use in space, and to assess the specific power and other attributes of that design. The resultant design is highly modular, consisting of standard RTG slices, each producing $\sim 24$ watts at the desired output voltage of 28 volt. Thus, the design could be adapted to various space missions over a wide range of power levels, with little or no redesign. Each RTG slice consists of a 250-watt heat source module, eight multicouple thermoelectric modules, and standard sections of insulator, housing, radiator fins, and electrical circuit. The design makes it possible to check each thermoelectric module for electrical performance, thermal contact, leaktightness, and performance stability, after the generator is fully assembled; and to replace any deficient modules without disassembling the generator or perturbing the others. The RTG end sections provide the spring-loaded supports required to hold the free-standing heat source stack together during launch vibration. Detailed analysis indicates that the design offers a substantial improvement in specific power over the present generation of RTGs, using the same heat source modules.
\end{abstract}




\section{INTRODUCTION}

Over the past 20 years, Radioisotope Thermoelectric Generators (RTGs), developed under the aegis of the Department of Energy's Space and Terrestrial Systems Division (DOE/STS) or its predecessors, have been successfully used in over twenty space missions [1]. During that period, they have undergone a steady process of improvement, and this evolutionary process is continuing. Its primary goal is to increase the power-to-weight ratio of RTGs, while retaining or improving their existing safety and reliability. When promising new technological and/or design options become available, conceptual designs to take advantage of those options are prepared and analyzed. If these advanced RTG designs show sufficient likelihood of success and ultimate payoff, their development is pursued for use in future missions.

The RTGs most recently flown in space are the Multi-Hundred-Watt (MHW) units developed and built by GE [2, 3]. These units were used to power the Voyager spacecraft, which recently sent back much new information about Jupiter, Saturn, and their moons, and which is slated to fly by Uranus before leaving the solar system. A total of 10 MHW RTGs have been flown in space, and all nave exhibited excellent performance stability [4].

After the MHW units, DOE/STS pursued the development of a new, modular heat source, designated as the General-Purpose Heat Source (GPHS) because of its applicability to a wide range of energy conversion systems and power levels. The GPHS module had first been designed by Los Alamos, with analytical support from Battelle; it was later considerably modified by Fairchild [5], and is currently under development by GE with major contributions by a number of other organizations.

The latest generation of RTGs, currently under construction, is designated as the GPHS/RTG because it is built around the new General-Purpose Heat Source. It started out as a conceptual design by Fairchild [6] for DOE/STS, which was partially modified by GE [7], the DOE flight system contractor. The GPHS/RTGs are being built for use on the NASA Galileo mission, to orbit Jupiter, study its moons, and send a probe into its atmosphere; and for the NASA/ESA Solar-Polar Mission, to study the region outside the earth's ecliptic plane and above the sun's poles. First launch of the GPHSIRTG is currently scheduled for 1985 . 
Consideration has lately been given to more advanced and much lighter RTG designs, for use on future missions. To help this effort, DOE/STS - in the summer of 1980-asked Fairchild to prepare a new RTG design taking optimum advantage of new materials, fabrication techniques, and design concepts developed in the past few years, and to carry out an analysis to assess its potential. This work is described here.

As will be seen, the conceptual design which emerged from the Fairchild study incorporates the modular GPHS heat source mentioned earlier; a new thermoelectric material developed by Syncal (with the support of NASA/JPL and DOE/STS); a multicouple thermoelectric module concept pioneered by Syncal, demonstrated by them in a DOE/STS program, and more recently modified by Fairchild; a multi-foil thermal insulation system developed by Thermoelectron [8]; and novel concepts for the thermoelectric converter arrangement and for the heat source structural supports, developed by Fairchild.

The RTG design evolved by Fairchild is designated as the Modular Isotopic Thermoelectric Generator (MITG) for reasons explained in Section 2, in which an overview of the design is presented. Section 3 discusses the new thermoelectric material and its properties, and Sections 4 through 6 describe the thermoelectric module design and fabrication. Sections 7 through 13 present a detailed discussion of various generator components and assembly procedures. Finally, Sections 14 through 18 summarize the salient parameters of an illustrative 282-watt MITG design, and compare its specific power with that of past and present RTGs. 


\section{OVERVIEW OF MITG DESIGN}

The principal distinguishing feature of the MITG design is its modularity. Except for its end sections, the generator consists of a number of identical slices of standard design, each producing approximately 24 watt at 28 volt. Thus, the basic design is adaptable to a wide range of power levels, since the output power can be scaled in 24-watt steps by varying the number of standard generator slices, generally without any other design changes. In addition, any intermediate power level can be provided by minor modification of the radiator fin dimensions.

This scalability of the MITG is a major advance over earlier RTG designs. In the MHW generator, a change in power level would have required a major redesign and requalification of the heat source. And even with the GPHS generator, which uses the same modular heat source as the MITG, changing the power level would generally require major changes in the thermoelectric couples and circuit to maintain the same output voltage.

Each slice of the MITG generator consists of one GPHS heat source module surrounded by eight thermoelectric modules, as well as standardized sections of thermal insulation, housing, radiator fins, and electrical circuit. When varying the number of slices in the generator, the only item which may have to be redesigned is the housing. For very long generators, the cantilevered housing may require a thicker wall to withstand the bending moments caused by transverse g-loads.

After the complete converter is assembled and an electrical heater is inserted, the thermoelectric modules can be individually checked for electrical performance, for thermal contact to the generator housing, for hermeticity of their C-ring seals, and for performance stability during extended operation. If any thermoelectric modules are found to be deficient, they can be replaced without disassembling the generator or perturbing the other modules. These features are not available in past or present RTG designs. In those, it is not possible to check the performance of individual thermoelectric couples; and even if a deficient one could be identified, it could not be replaced without major disassembly of the generator.

The final distinguishing feature of the MITG design is that its electrical circuit is lorated outside the generator housing. The series-parallel circuit permits high redundancy against open circuits, and-since the wiring is outside the generator-the chance of shorts-to-ground is minimized. Finally, if it were desired to produce a higher output voltage for all or part of the payload, the circult could be arranged to do so (e.g., up to $180 \mathrm{~V}$ at $300 \mathrm{~W}$ ). This option also was unavaliable in previous RTG designs. 
Figure 1 shows a sectioned view of the General-Purpose Heat Source (GPHS) module, which is at the center of each generator slice. The radioisotope fuel capsules are surrounded by a variety of safety provisions to ensure fuel containment under all credible accident conditions. Each heat source module has overall dimensions of roughly $3.8 \times 3.7 \times 2.1$ inches, and weighs $3.2 \mathrm{lbs}$. As can be seen, there are four 62.5 -watt $238 \mathrm{PuO}_{2}$ fuel pellets, giving a total thermal power of $250 \mathrm{~W}$ per heat source module.

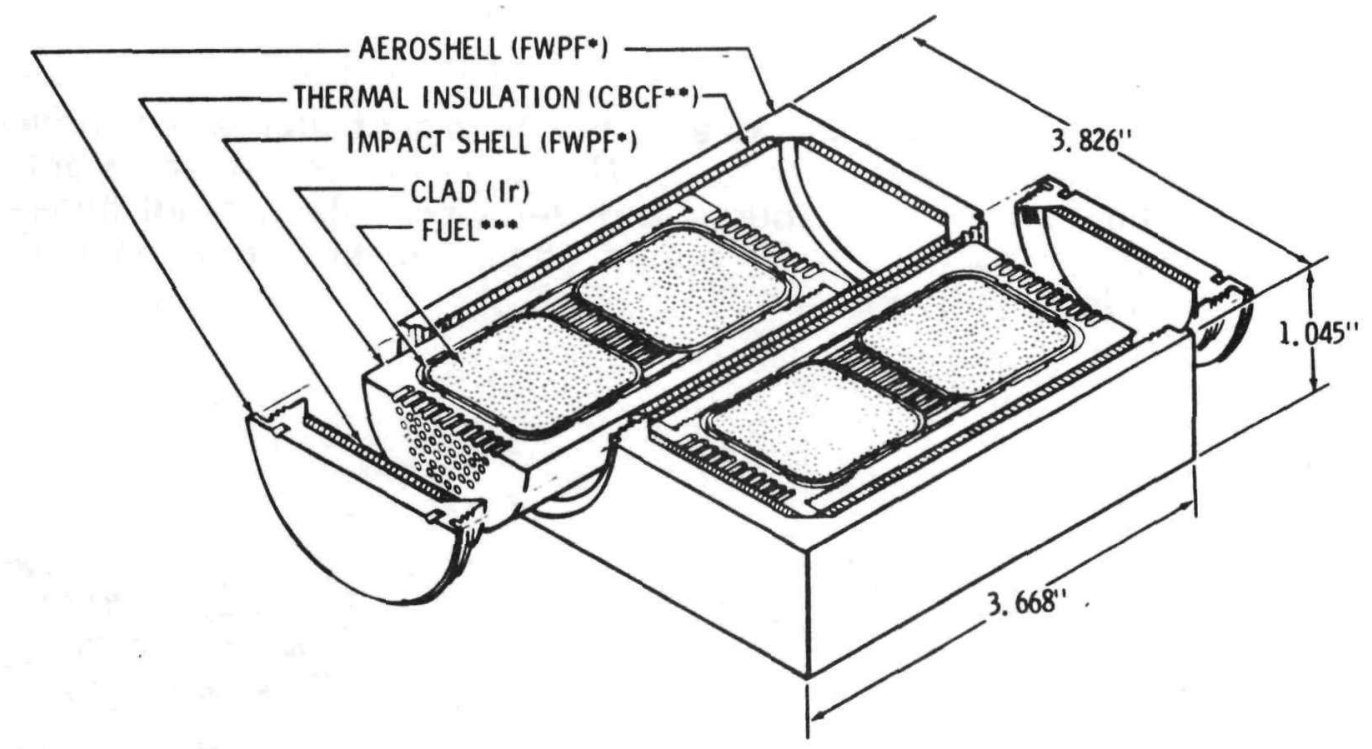

-Fine-Weave Pierced Fabric, a 90\%-dense 3D carbon-carbon composite

* Carbon-Bonded Carbon Fibers, a 10\%-dense high-temperature insulator **62. 5-watt ${ }^{238} \mathrm{PuO}{ }_{2}$ pellet

Figure 1. General-Purpose Heat Source Module (250 Watt) Sectioned at midplane

Each fuel pellet is contained in an iridium clad, which is expected to deform - but not breach-upon earth impact. The fuel capsules are protected by impact shells, which are designed to absorb energy by crush-up during impact; and the impact shells are protected by an aeroshell, which is designed to ablate but retain its integrity during the reentry heat pulse. There are two fuel capsules per impact shells, and two impact shells per aeroshell. Both the impact shells and the aeroshell are made of the same graphitic material, Fine-Weave Pierced Fabric (FWPF). This is a three-dimensional carbon-carbon composite of high density and strength. 
Between the impact shells and the aeroshell is a layer of high-temperature thermal insulation. This consists of CarbonBonded Carbon Fibers (CBCF), a low-density structure with excellent insulating properties at high temperatures. The insulator is designed to prevent the fuel capsules from overheating during the reentry heat pulse and from overcooling during subsequent atmospheric descent. A much fuller explanation of the GPHS design rationale was presented in a recent paper [5].

Figure 2 shows a sectioned view of the heat source surrounded by eight thermoelectric modules. The latter are described in detail in Section 4. The heat source and the thermoelectric hot shoes are separated by 0.35 -inch vacuum gaps, and heat transfer between them is by radiation only.

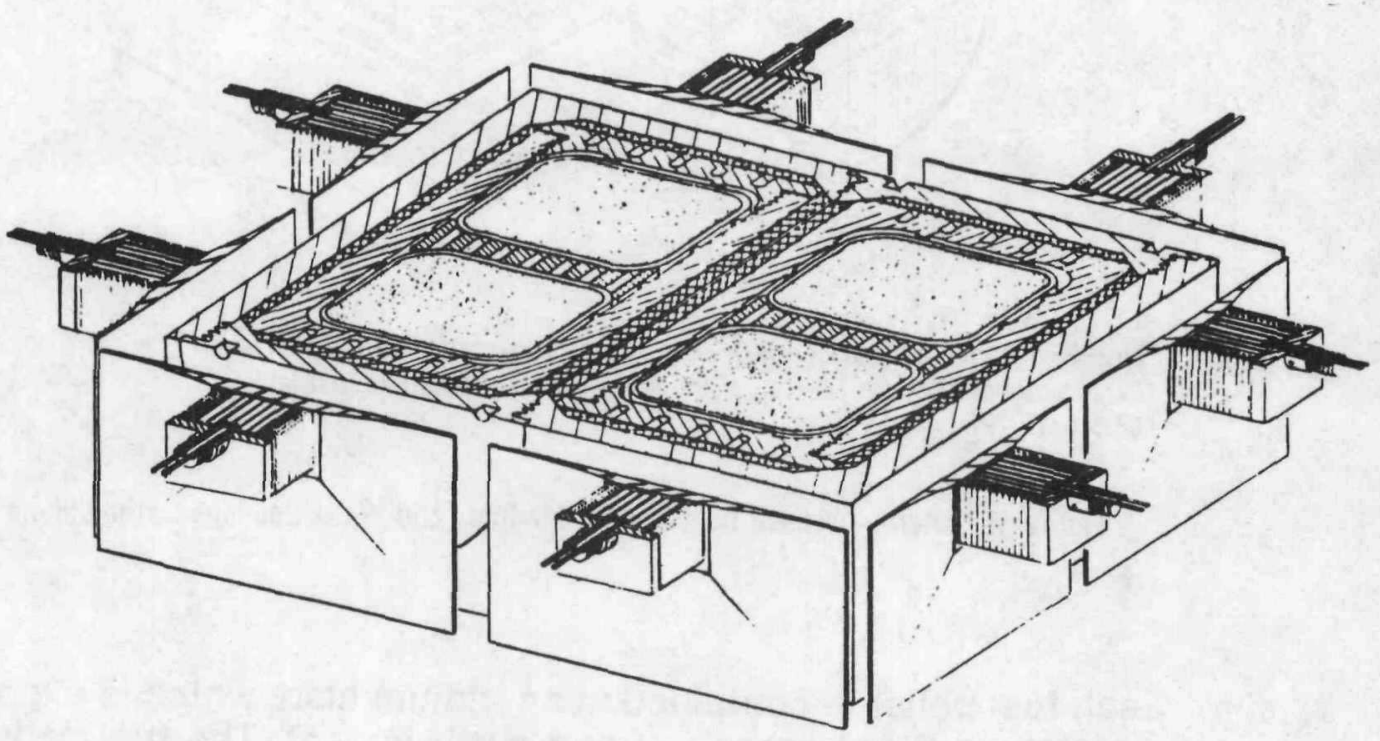

Figure 2. Heat Source Module with its Eight Thermoelectric Modules, Sectioned at midplane 
Figure 3 shows a complete generator slice, split at its midplane to display its interior components. In addition to the heat source and thermoelectric modules depicted previously, the figure shows the thermal insulation, the generator housing with attached radiator fins, and the external electrical circuit loop with its 28-volt output. Each of these subsystems is discussed in detail below.

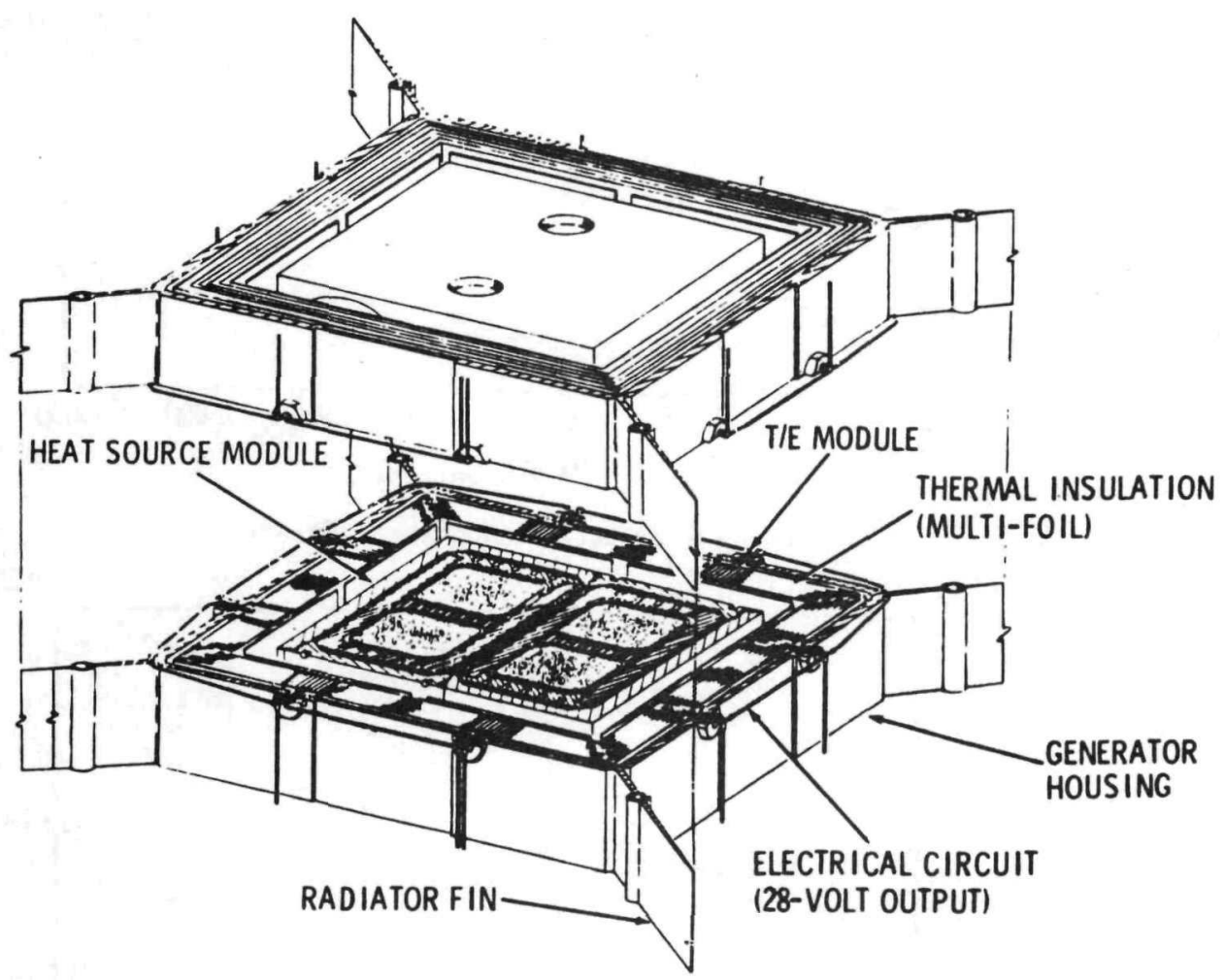

Figure 3. Typical Generator Slice ( $\sim 24$ Watts at 28 volts) Split at midplane, to display internal components 


\section{THERMOELECTRIC MATERIAL PROPERTIES}

The new thermoelectric material developed by Syncal is obtained by the addition of $5 \%$ gallium phosphide to the silicon-germanium alloy used in the MHW and GPHS RTGs. Figures 4, 5, and 6 show the temperature dependence of the new material's Seebeck coefficient, electrical resistivity, and thermal conductivity, as reported by Syncal. Each figure presents separate curves for the $\mathrm{N}$ - and P-material.

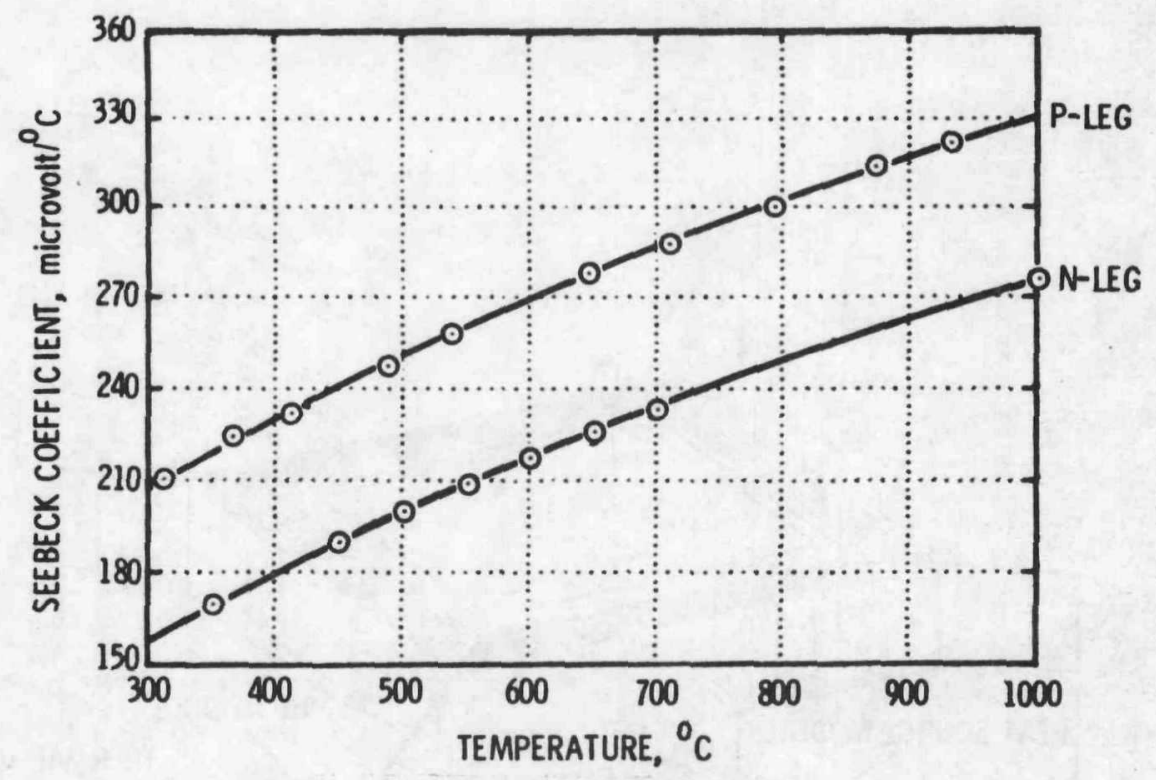

Figure 4. Seebeck Coefficient of SiGe/GaP

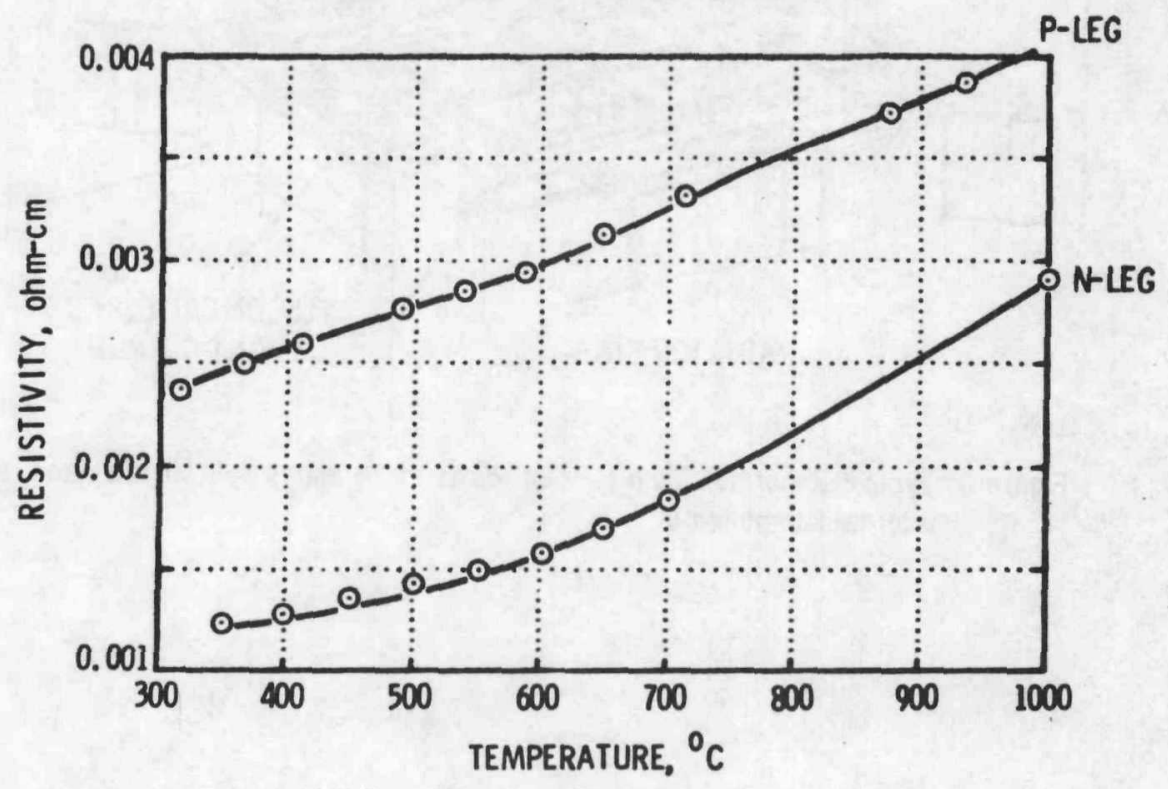

Figure 5. Electrical Resistivity of SiGe/GaP 
The Seebeck coefficients and electrical resistivities shown in Figures 4 and 5 are very similar to the corresponding values of the standard SiGe material. Where the GaP additive makes a big difference is in the thermal conductivity. This is illustrated in Figure 6, which compares the conductivity of the new material (solid curves) with that of the standard material (dashed curves). As can be seen, the new material has a substantially lower conductivity, which accounts for its much higher conversion efficiency.

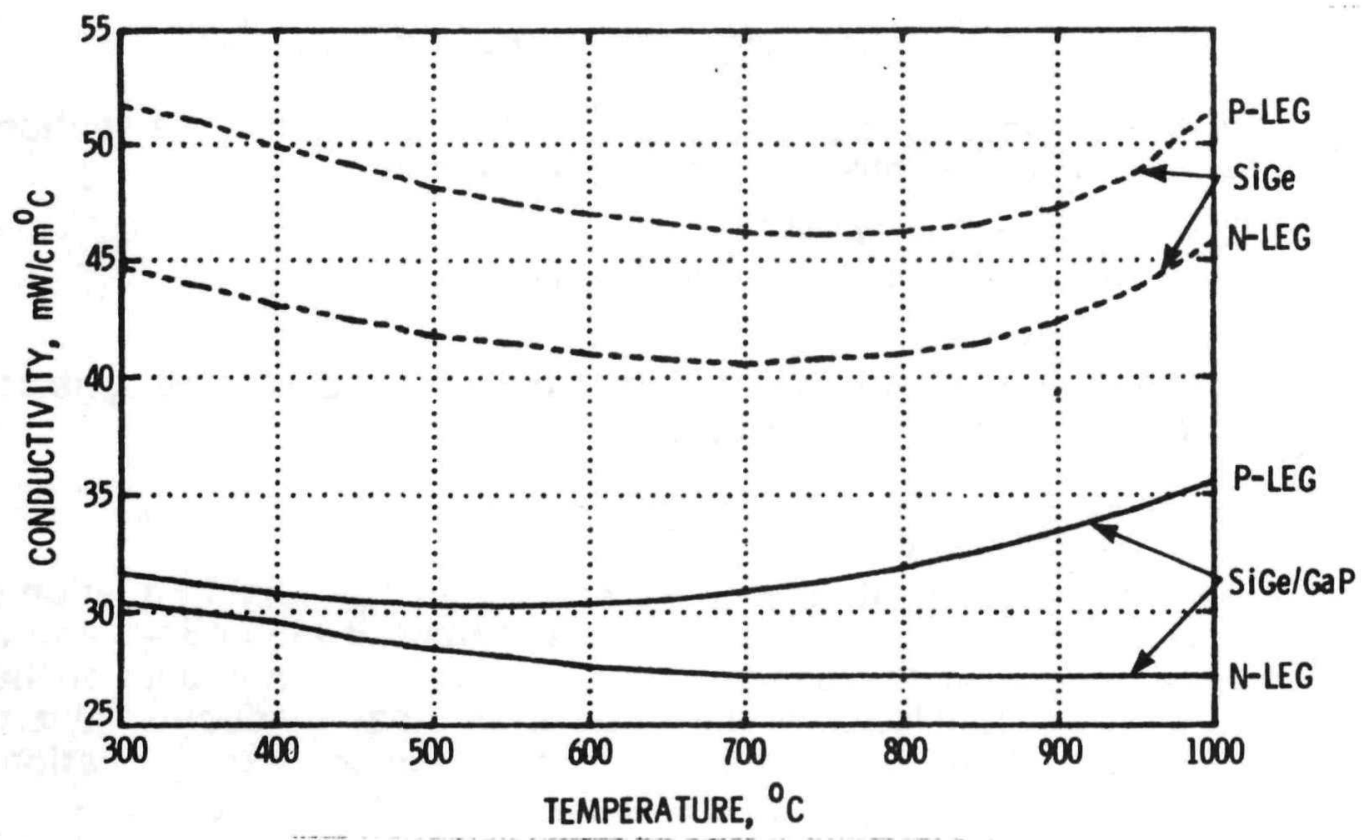

Figure 6. Thermal Conductivity of T/E Materials 
The temperature-dependent values of the Seebeck coefficient $\alpha$, conductivity $x$, and resistivity $Q$ plotted in Figures 4,5 , and 6 can be used to compute temperature-averaged values $\bar{\alpha}, \bar{x}, \overline{0}$, using the averaging techniques recommended in [9]. For an illustrative hotjunction temperature $T_{H}=1000^{\circ} \mathrm{C}$ and cold-junction temperature $T_{C}=300^{\circ} \mathrm{C}$, we obtain:

$$
\begin{aligned}
\bar{\alpha} & =\int_{300}^{1000}\left(\alpha_{N}+\alpha_{P}\right) d T / 700=0.4968 \times 10^{-3} \text { volt } /{ }^{\circ} \mathrm{C}, \\
\bar{x}_{N} & =\int_{300}^{1000} x_{N} d T / 700=0.0278 \mathrm{watt} / \mathrm{cm}^{\circ} \mathrm{C}, \\
\bar{x}_{P} & =\int_{300}^{1000} x_{F} \cdot d T / 700=0.0316 \mathrm{watt} / \mathrm{cm}^{\circ} \mathrm{C}, \\
\bar{\varrho}_{N} & =\int_{300}^{1000} x_{N} Q_{N} d T / 700 \bar{x}_{N}=0.1794 \times 10^{-2} \mathrm{ohm}-\mathrm{cm}, \text { and } \\
\bar{\varrho}_{P} & =\int_{300}^{1000} x_{P} \varrho_{P} d T / 700 \bar{x}_{P}=0.3177 \times 10^{-2} \mathrm{ohm}-\mathrm{cm} .
\end{aligned}
$$

A thermoelectric couple with legs of length $L$ and cross-sectional areas $A_{N}$ and $A_{P}$ has a thermal conductance

$$
K=\left(\bar{x}_{N} A_{N}+\bar{x}_{P} A_{P}\right) / L
$$

and an internal electrical resistance

$$
R=\left(\bar{Q}_{N} / A_{N}+\bar{Q}_{P} / A_{P}\right) L \text {. }
$$

If the couple operates at a current $/$ and an output voltage $V$, its conversion efficiency $\eta$ is given by [10]:

$$
\eta=\frac{I V}{K \Delta T+\bar{\alpha} T_{H} I-1 / 2 I^{2} R},
$$

where $\Delta T$ represents the temperature drop $T_{H}-T_{C}$. In Equation (8), the numerator represents the power output of the couple, and the three terms in the denominator represent the hot-junction heat input rate, consisting of the zero-current heat conduction plus the Peltier cooling rate, less one half of the ohmic heat dissipation in the thermoelectric legs. (The other half ends up at the cold-junction.) Since the open-circuit voltage of the couple is $\alpha \Delta T$, its output voltage $V$ and current $/$ are related by the voltage balance

$$
\bar{\alpha} \Delta T=I R+V \text {. }
$$

Combining Equations (8) and (9) to eliminate I, we obtain

$$
\eta=\frac{V(\bar{\alpha} \Delta T-\eta)}{K R \Delta T+\bar{\alpha} T_{H}(\bar{\alpha} \Delta T-V)-1 / 2(\bar{\alpha} \Delta T-V)^{2},}
$$

which is the general solution for the efficiency of a couple, before optimization of its leg dimensions and output voltage. 
The leg dimensions are optimized by minimizing the product $K R$ in Equation (10). As seen from Equations (6) and (7), the value of this product is given by

$$
K R=\left(\bar{x}_{N}+\bar{x}_{P}\right)\left(\bar{\varrho}_{N}+\bar{\varrho}_{P}\right)=2.953 \times 10^{-4} \text { watt ohm } /{ }^{\circ} \mathrm{C}
$$

for the case of equal leg areas, and can be reduced to its minimum value

$(K R)_{\min }=\left[\left(\bar{x}_{N} \bar{Q}_{N}\right)^{1 / 2}+\left(\bar{x}_{P} \bar{Q}_{P}\right)^{1 / 2}\right]^{2}=2.918 \times 10^{-4}$ watt ohm/ ${ }^{\circ} \mathrm{C}$

by optimizing the leg area ratio:

$\left(A_{N} / A_{P}\right)_{\text {opt }}=\left[\left(\bar{x}_{P} / \bar{x}_{N}\right) /\left(\bar{\varrho}_{P} / \bar{Q}_{N}\right)\right]^{1 / 2}=0.80$,

Inserting Equation (12) into (10) gives the efficiency $\eta^{\prime}$ of a couple with optimized leg areas:

$\eta^{\prime}=\frac{V(\alpha \Delta T-V}{\left[\left(\bar{x}_{N} \bar{\varrho}_{N}\right)^{1 / 2}+\left(\bar{x}_{P} \bar{Q}_{P}\right)^{1 / 2}\right]^{2} \Delta T+\bar{\alpha}_{H}\left(\bar{\alpha} \Delta T-V-1 / 2\left(\bar{\alpha} \Delta T-V^{2}\right.\right.}$.

The above expression is maximized by setting the load voltage to

$$
V_{\text {opt }}=\bar{\alpha} \Delta T /\left[1+(1+\bar{z} \bar{T})^{-1 / 2}\right]=0.199 \text { volt, }
$$

where $\bar{T}$ is the arithmetic average of the hot- and cold-junction temperatures, and $Z$ is a temperature-averaged figure of merit, defined by

$$
\left.\bar{z}=\left\{\bar{\alpha} /\left(\bar{x}_{N} \bar{Q}_{N}\right)^{1 / 2}+\left(\bar{x}_{P} \bar{Q}_{P}\right)^{1 / 2}\right]\right\}^{2}=0.000846^{\circ} \mathrm{K}^{-1} .
$$

Inserting Equation (15) into (14) gives the maximum material efficiency $\eta^{\prime \prime}$ of the couple, with optimized leg areas and optimized load voltage:

$$
\eta^{\prime \prime}=\left[(1+\bar{Z} \bar{T})^{1 / 2}-1\right]\left(T_{H}-T_{C}\right) /\left[\left(1+\bar{Z} \bar{T}^{1 / 2} T_{H}+T_{C}\right]=0.105 .\right.
$$

The efficiency value discussed above is called the material efficiency, to differentiate it from the couple efficiency. The latter takes account of imperfections in the thermoelectric legs and in the hot- and cold-junctions, and includes corrections for additional heat losses through other couple components, such as insulating coatings and wraps. The material efficiency for SiGe/GaP given by Equation (17) is substantially higher than the corresponding efficiency of 0.083 for standard SiGe, for the same hot- and coldjunction temperatures. This demonstrates the value of the GaP additive in the new material. 


\section{THERMOELECTRIC MODULE DESIGN}

Figure 7 shows an overview of the thermoelectric module (multicouple), with the insulation wrap around the thermoelectric legs omitted for clarity. The module receives a heat input of approximately 30 watt on its hot shoe. Most of this heat flows through the thermoelectric legs to the cold shoe. The legs have a hot-junction temperature of $1000^{\circ} \mathrm{C}$ (the same as in present-generation SiGe generators) and a cold-junction temperature of roughly $300^{\circ} \mathrm{C}$. The terminal leads of the thermoelectric module emerge through the stud of the cold shoe, delivering an output of less than 1 amp at 3.5 volt.

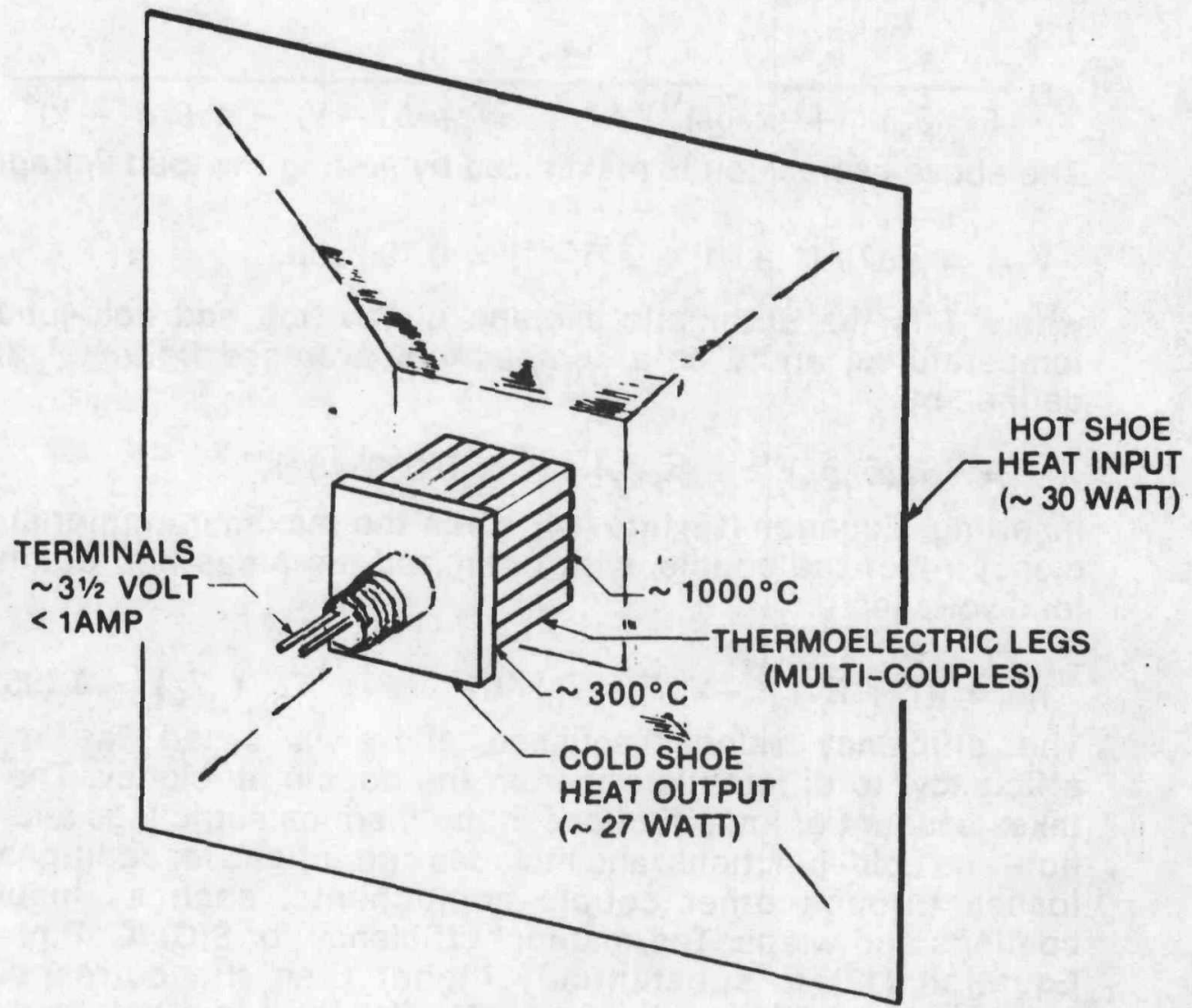

Figure 7. Thermoelectric Module (without insulation wrap) 
As can be seen, the thermoelectric module contains a multiplicity of legs. Assuming a desired output of 28 volt per generator slice, each of the eight series-connected thermoelectric modules must produce 3.5 volt. As shown in Equation (15),the optimum couple voltage for SiGe/GaP operating between hot- and cold-junction temperatures of $1000^{\circ}$ and $300^{\circ} \mathrm{C}$ is 0.199 volt. Thus, the 3.5 -volt module output can be produced by 18 couples or 36 legs per module. As will be seen, this optimum number of legs lends itself well to a multicouple design.

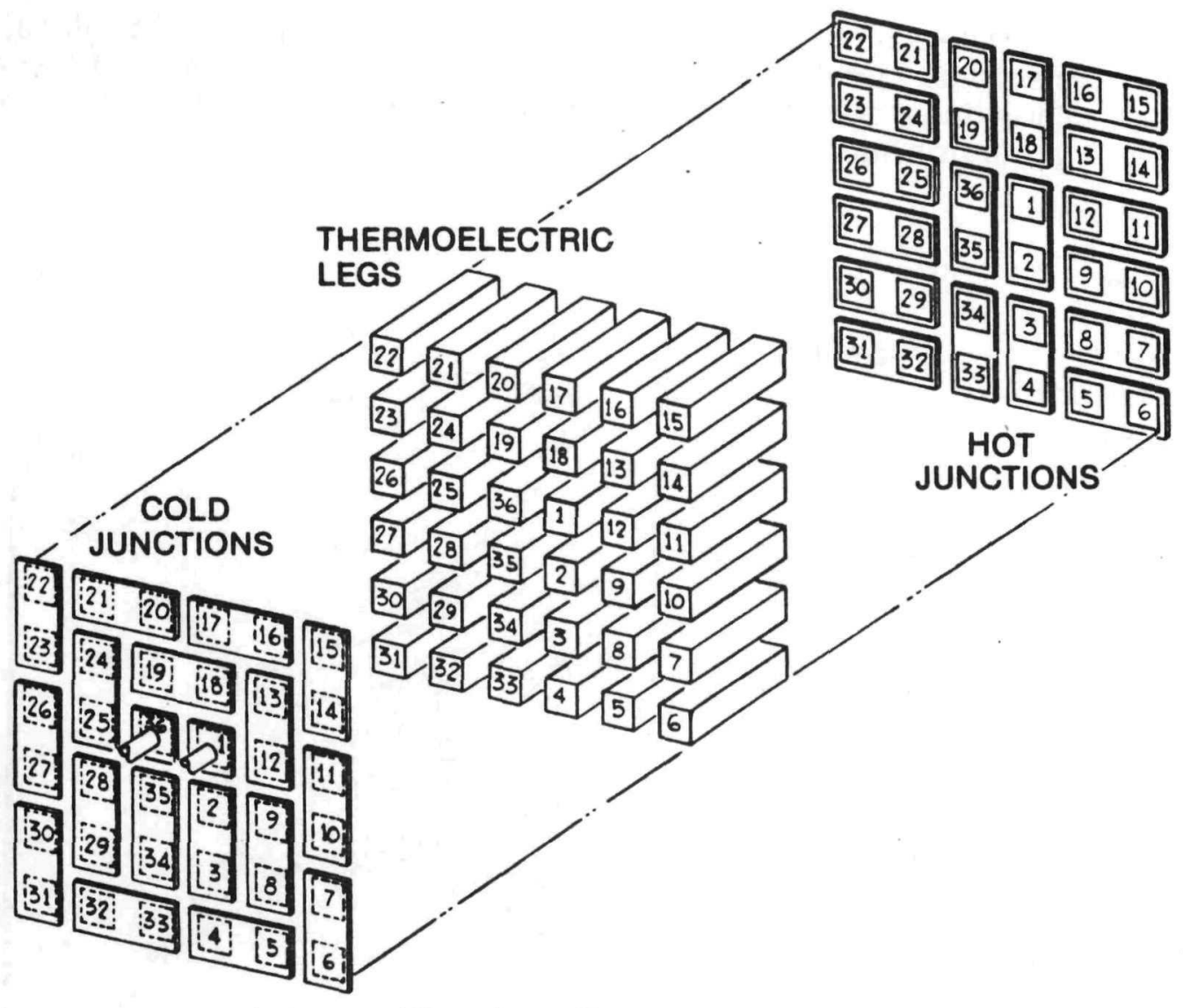

Figure 8. Multicouple Arrangement (Exploded View)

The arrangement of the module's 36 legs together with the required hot- and cold-junction connectors is shown in exploded view in Figure 8. Although the alternating $\mathbf{N}$ and $\mathrm{P}$ legs within a module are connected in series (as indicated by the sequential numbers), the overall MITG circuit is a highly redundant network, as will be explained in Section 10. As shown in Figure 8, the first and last legs are located at the center of the module, to facilitate the passage of the terminal leads through the cold-shoe stud, as illustrated earlier in Figure 7. 
Figure 9 shows the components of the thermoelectric module in exploded view, with the insulator wrap again omitted. The leg assembly, whose fabrication is discussed in the next section, forms a nearly cubical block of $0.30 \times 0.36 \times 0.36$ inches. The hot junctions consist of diffusion-bonded silicon wafers, and the cold junctions of a sputtered and photoetched tungsten coating. The 2"-square hot shoe and the cold shoe with integral stud are made of molybdenum, and the built-in terminal leads are nioblum wires, whose ends are brazed Into axial holes at the cold ends of the first and last thermoelectric legs. They are then coated with $\mathrm{SiO}_{2}$ glass, for electrical isolation from the cold shoe. The outer face of the cold shoe has a gold foil brazed to it, for improved thermal contact to the generator housing.

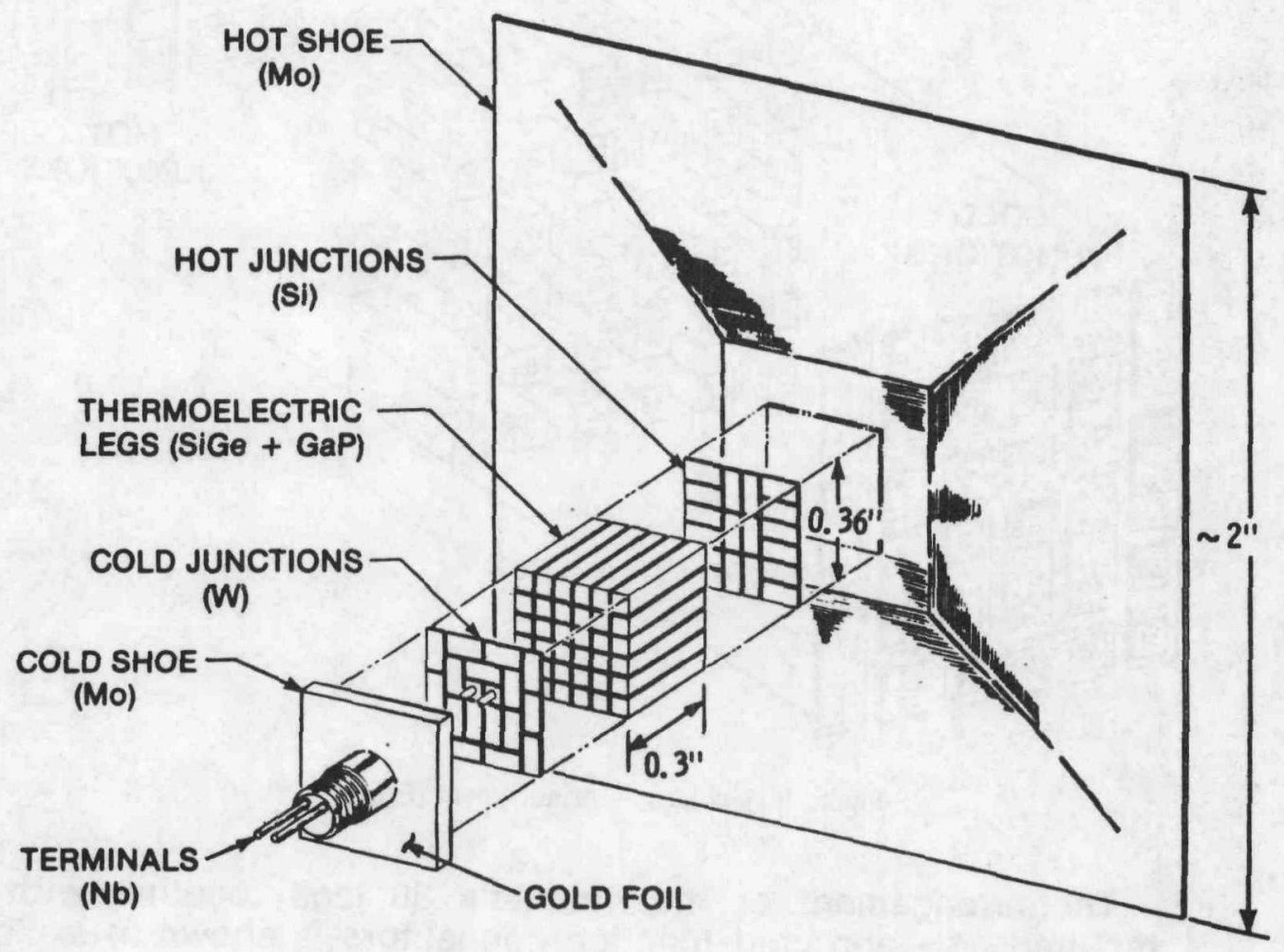

Figure 9. Thermoelectric Module Components (Insulators Not Shown) 
The figures presented thus far have shown all legs as having the same cross-sectional areas. However, as was shown earlier in Equation (13), the optimum $\mathrm{N}$-leg to $\mathrm{P}-\mathrm{leg}$ area ratio for this material is 0.80 . The resultant improvement in material efficiency is illustrated in Figure 10.

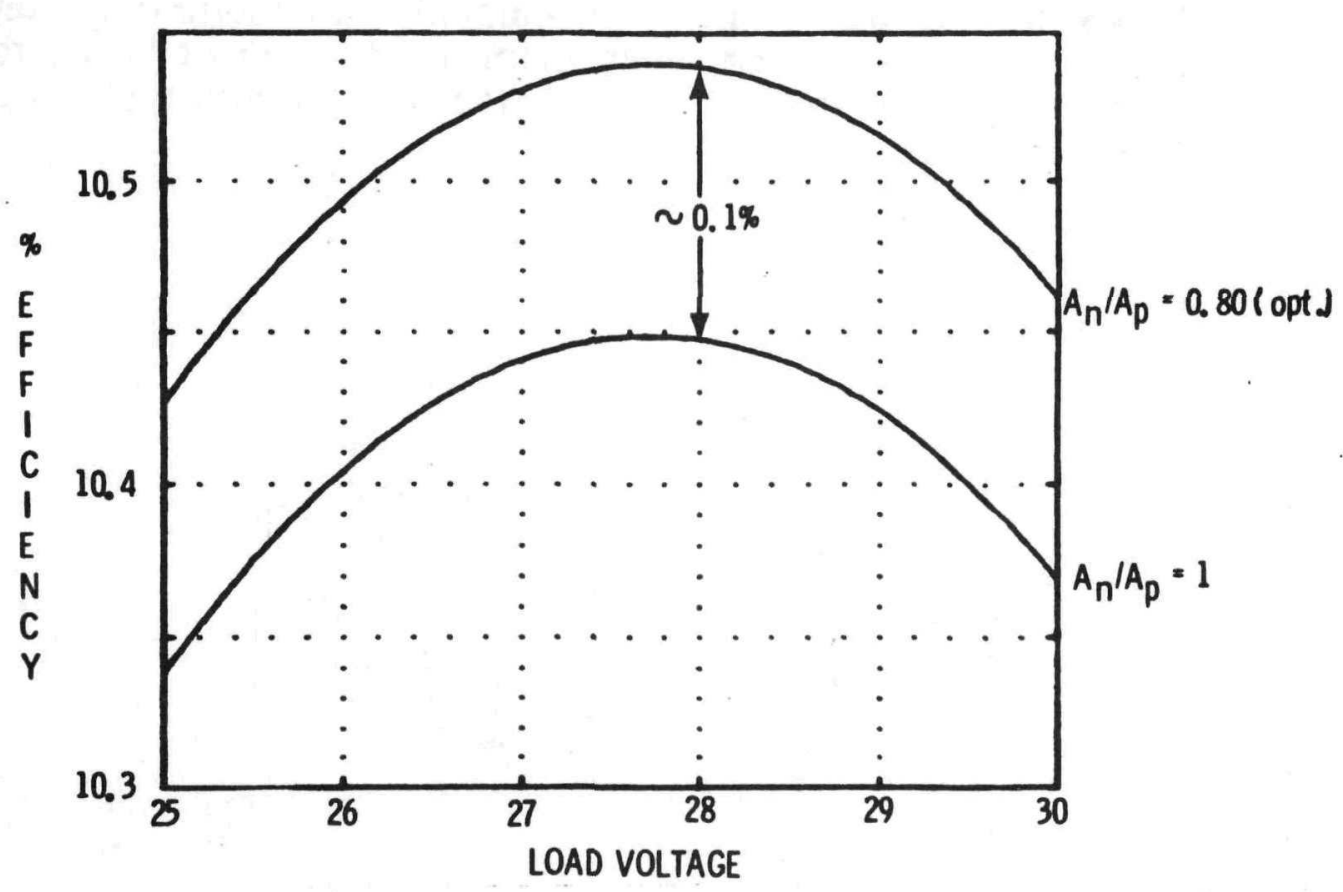

Figure 10. Effect of Leg Area Ratio on Thermoelectric Material Efficiency of SiGe/GaP

The curves show the variation of efficiency with load voltage, for the assumed hot- and cold-junction temperatures, and for 36 legs per module and 8 modules in series. The lower curve shows the material efficiency of a module with equal-area legs, computed by means of Equations (10) and (11); and the upper curve shows the efficiency of a module with the optimum leg area ratio of 0.80 , computed by means of Equation (14). As can be seen, optimization of the leg area ratio gains approximately $0.1 \%$ in efficiency. Thus, we conclude that the use of non-uniform leg area is beneficial, but would not be essential if it proved to be too difficult to implement. 


\section{THERMOELECTRIC MODULE FABRICATION}

Figure 11 illustrates the multicouple fabrication technique developed by Syncal, and shows that this technique would indeed be adaptable to the production of optimized leg areas. Starting at the top with blocks of $\mathbf{N}$ and $\mathrm{P}$ material, the first step is to cut slices of $0.051 "$ and 0.063 " thickness, respectively. These slices are individually coated with a glass layer consisting primarily of silica. Three slices of each type are assembled in an alternating array, and are fused together. The fused block is then sliced in a direction perpendicular to the bonded interfaces, to produce six slices of 0.057 " thickness.

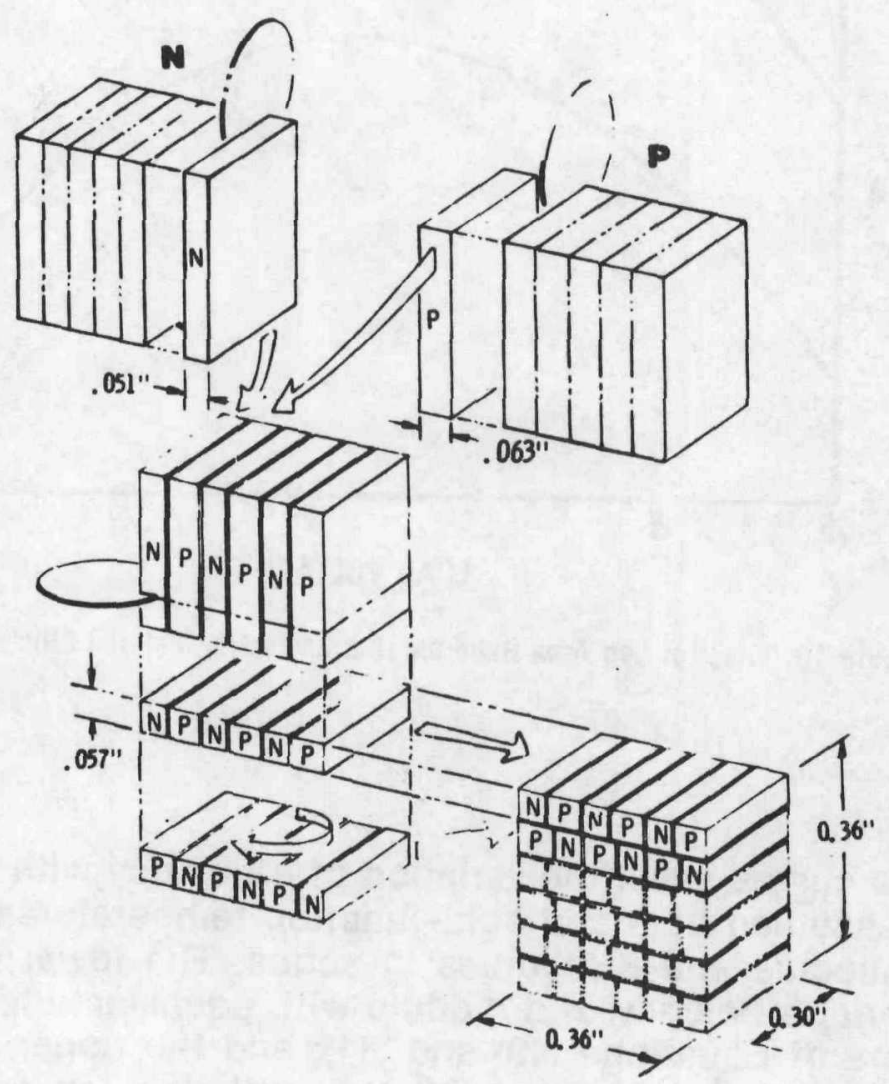

Figure 11. Fabrication of $T / E$ Module with Optimized $A_{n} / A_{p}$ 
These slices, containing alternating $\mathrm{N}$ and $\mathrm{P}$ legs, are again coated with sillica glass, and six such slices are assembled into a block in which the $\mathrm{N}$ and $\mathrm{P}$ legs form a checkerboard pattern, as shown. The slices forming this block are joined together by fusing the intervening glass layers, and the faces of the block are subsequently coated with the same glass material. Thus, the glass coating acts both as a bond and as an electrical insulator. In addition, as will be discussed later, it also serves to supress the sublimation of silicon from the hot ends of the thermoelectric legs.

The above fabrication sequence, as implemented (for equal-area legs) by Syncal Corporation, is depicted in Figures 12.

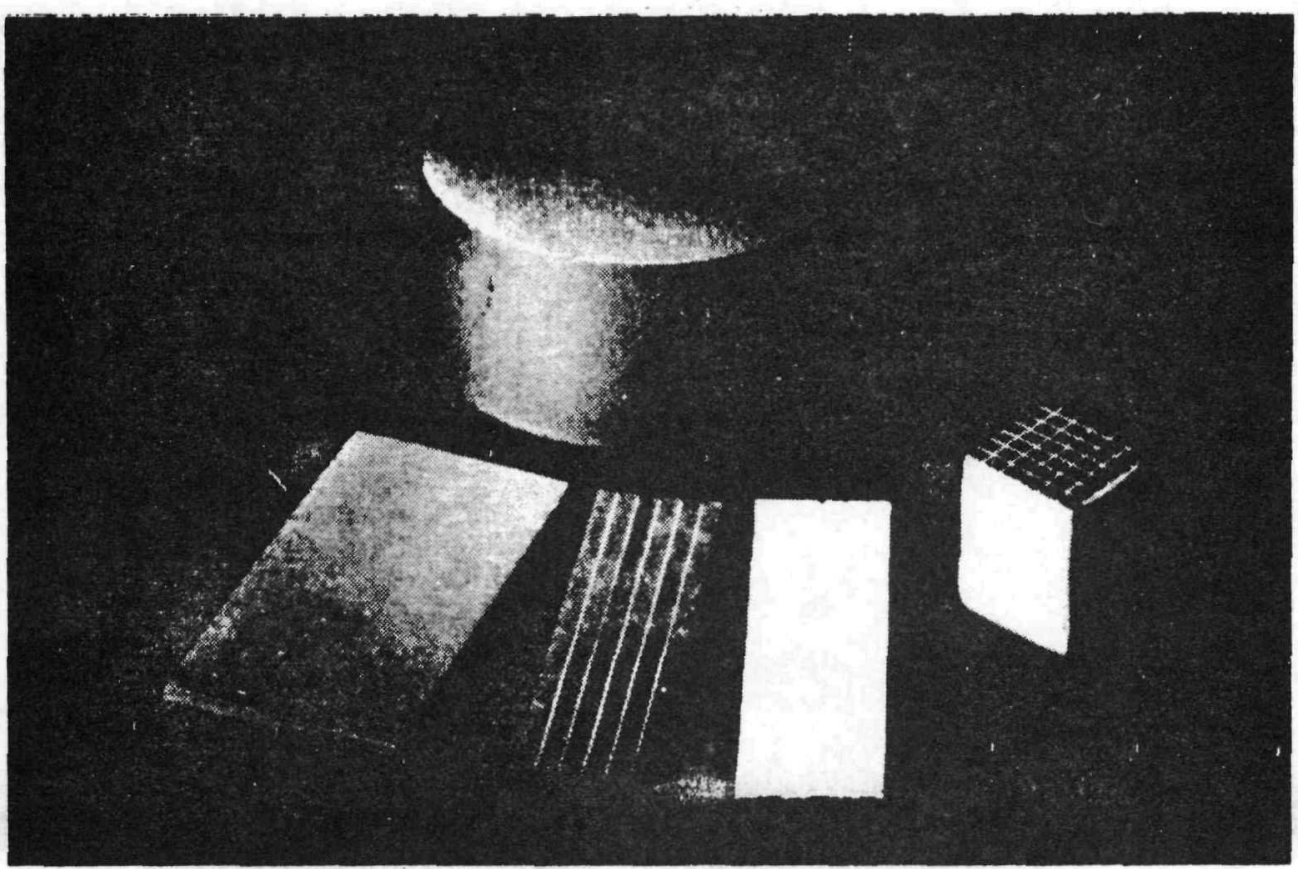

Figure 12. Multi-Leg Fabrication Sequence 
As illustrated in Figure 13, the two uninsulated end faces of the block of thermoelectric legs are then covered with hot junctions and cold junctions, and the two junction faces are covered with additional glass layers, which are used to insulate them from and bond them to the hot and cold shoes of the module. A static tensile test at Syncal has demonstrated that the glass bond to the hot shoe is quite strong, in fact stronger than the thermoelectric legs themselves. But no dynamic tests to demonstrate the ability of the bond to survive launch vibration have been carried out as yet. In the author's opinion, this is the most critical unresolved feasibility issue of the MITG design at present.

It should be noted that all of the thermoelectric elements and all circuit elements within the generator are completely enclosed by glass coatings. In contrast to previous RTGs, the MITG hot shoes and cold shoes are electrically isolated from the thermoelectric circuit. Therefore, contact between them and the electrically grounded metallic multifoil insulation is not harmful, and the need for the previously used ceramic insulator wafers is eliminated. This saves weight, and enhances the reliability of the generator by minimizing the chance for internal shorting (e.g., by loose foil insulation particles). 


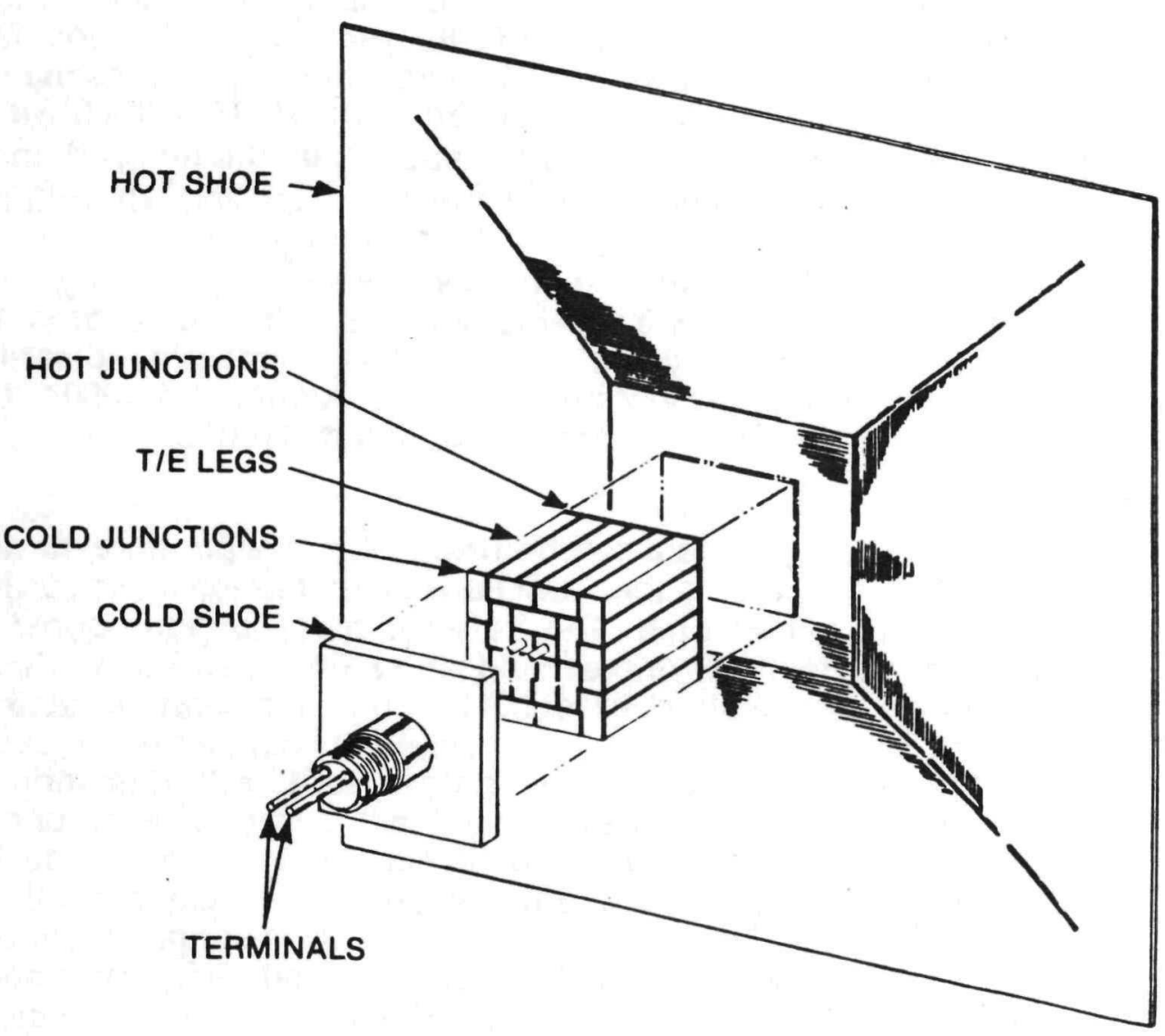

Figure 13. Thermoelectric Circuit Assembly Ready for Glass-Bonding to Hot and Cold Shoes 
In addition to its bonding and insulating function, the silica glass coating can also serve as a sublimation suppressant, since it completely covers the SiGe legs and the Si hot shoes. Silicon sublimation from the hot end of the thermoelectric legs is an important concern, because it can act as a life-limiting mechanism. In previous generators like the MHW and GPHS/RTGs, the SiGe legs were coated with silicon nitride to reduce sublimation [11]. In the present design it is expected that the glass coating will fulfill this suppression function for the required operating life.

Only limited confirmatory tests have been completed by Syncal thus far. Figure 14 shows a log-log plot of sublimation expressed in mils versus test time in years. The test data points are indicated by open circles. Data were obtained for bare coupons at $1000^{\circ}, 1050^{\circ}$ and $1100^{\circ} \mathrm{C}$, and for glass-coated coupons at $1100^{\circ} \mathrm{C}$.

As can be seen, all data can be fitted with straight lines having a slope of 0.88 , suggesting that cumulative sublimation is proportional to the 0.88 power of time. The data for bare coupons show that sublimation is 15 times more rapid at $1100^{\circ} \mathrm{C}$ than at the desired hot-junction temperature of $1000^{\circ} \mathrm{C}$. This acceleration factor is important, because it makes it possible to simulate the desired 7 -year life in more reasonable test times. The $1100^{\circ} \mathrm{C}$ data indicates that the glass coating reduces the sublimation rate by a factor of 65 . Assuming that the log-log curve fit can be extrapolated to long times, that the same temperature acceleration factor applies to coated samples, and that the $1100^{\circ} \mathrm{C}$ sublimation suppression factor also applies at $1000^{\circ} \mathrm{C}$, we can infer the dashed curve for a coated sample at $1000^{\circ} \mathrm{C}$. For a seven-year mission, this curve predicts a sublimation loss 0.17 mils, which is only $6 \%$ of the assumed $3-\mathrm{mil}$ coating thickness on the outside of the thermoelectric legs. While these results are encouraging, more and longer tests are clearly needed to confirm this prediction. 


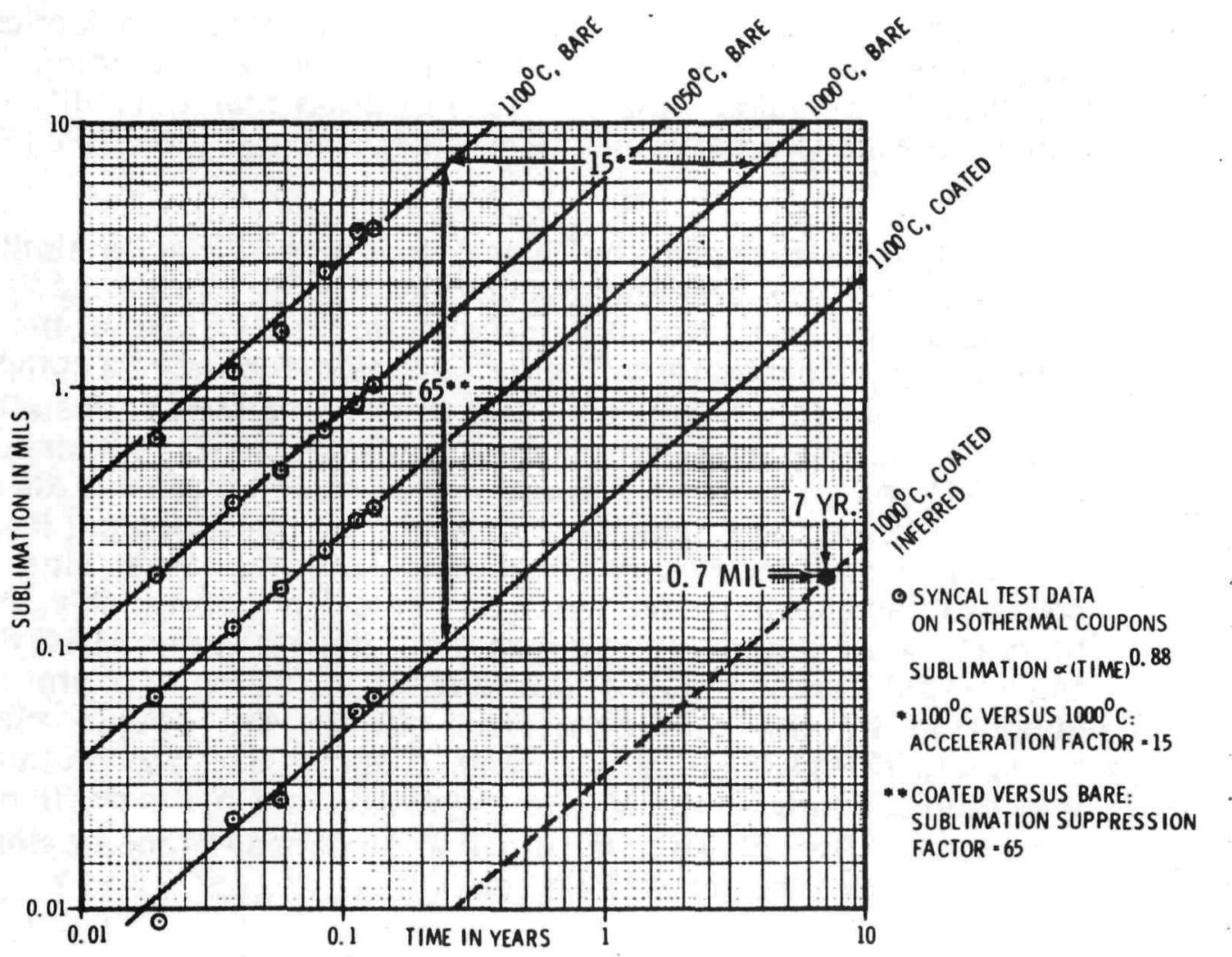

Figure 14. Effect of $\mathrm{SiO}_{2}$ Glass Coating on SiGe-GaP Sublimation 
Figure 15 shows a photograph of a completed multicouple module, produced by Syncal by means of the above-described fabrication procedure. The experience gained there is quite encouraging. Three multicouple modules were made, with good reproducibility. Each exhibited excellent performance stability during the $\mathbf{4 0 0 0}$ test hours to date.

The modules produced by Syncal are generally quite similar in their arrangement and dimensions to the MITG thermoelectric module design, but differ in four respects: first, instead of the rigid cold-shoe stud called for by the MITG design, they used a compliant cold-end heat shunt, similar to that used in the MHW generator. As will be seen, this compliant mounting arrangement is not needed in the MITG design. Second, instead of the brazed terminal leads at the center of the leg assembly postulated in the MITG design, they had their leads spot-welded to the outside of two corner legs. Such spot-welded side leads are much harder to fabricate reliably, and are more prone to failure during subsequent flexing. In the MITG design the central terminal leads are brazed in place and are rigidly supported by the cold-shoe stud. Third, the couple pictured contained 42 legs of equal area, instead of the 36 legs with optimized area ratio assumed in the MITG design. And fourth, the multicouples produced by Syncal had much smaller hot shoes ( 1 " square) than the 2" square hot shoe of the MITG design. 


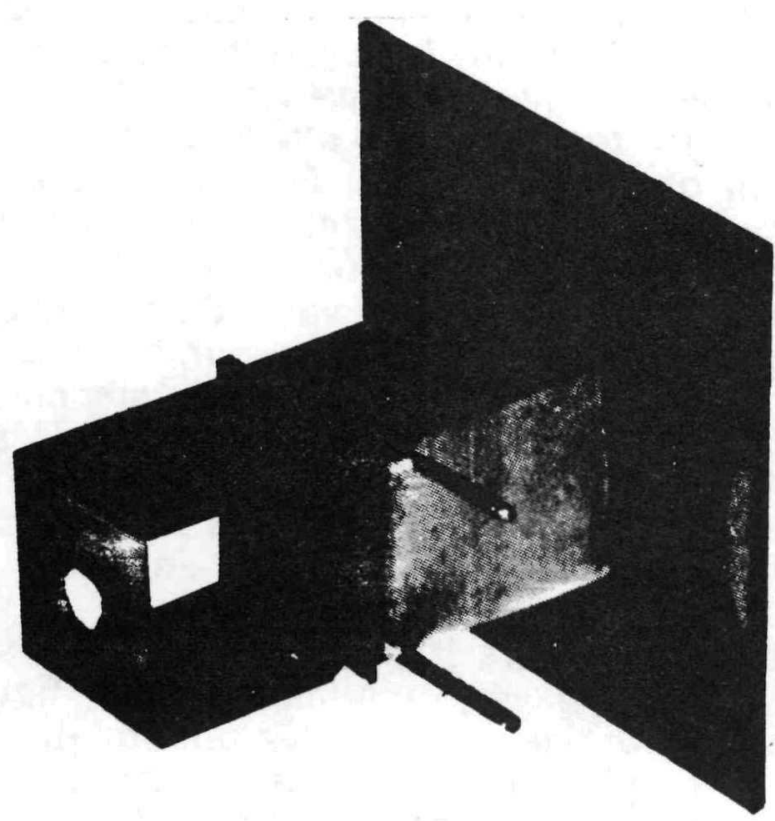

Figure 15. Multi-Couple Module Produced by Syncal 


\section{THERMOELECTRIC HOT SHOE}

The unusually large size of the MITG hot shoe makes its design of critical importance, not only because the hot shoe is the principal weight component of the thermoelectric module, but also because the more it weighs the higher the stress on the hot-junction bonds during launch of the spacecraft. For these reasons, we wish to minimize the weight of the hot shoe by reducing its thickness. However, the thinner the hot shoe is, the higher the temperature gradients required for heat flow towards the thermoelectric legs, and the higher the temperature of the heat source surface for a given hotjunction temperature. Heat source surface temperature, however, is presently limited to $1100^{\circ} \mathrm{C}$, because of concern about excessive grain growth in the iridium fuel capsule at higher heat source temperatures. The problem, therefore, is to minimize the hot-shoe weight for the specified hot-junction and heat source surface temperatures.

As illustrated in Figure 16, the use of a hot shoe of uniform thickness would be very inefficient. The figure shows the temperature map for one quadrant of a uniform-thickness hot shoe. The origin of the plot represents the center of the thermoelectric legs, and the dashed line denotes the boundary of those legs. For a $1000^{\circ} \mathrm{C}$ hot junction, we calculate a temperature drop of $20^{\circ} \mathrm{C}$ through the glass bond layer, giving a temperature of $1020^{\circ} \mathrm{C}$ at the center of the hot shoe. It was found that a uniform hot shoe thickness of 31 mils is required to give a heat source temperature of $1100^{\circ} \mathrm{C}$. Such a hot shoe would weigh 20.5 grams, or $4.3 \mathrm{lb}$ for the 96 hot shoes in a typical twelve-slice RTG. This would be a significant addition to the system weight. 


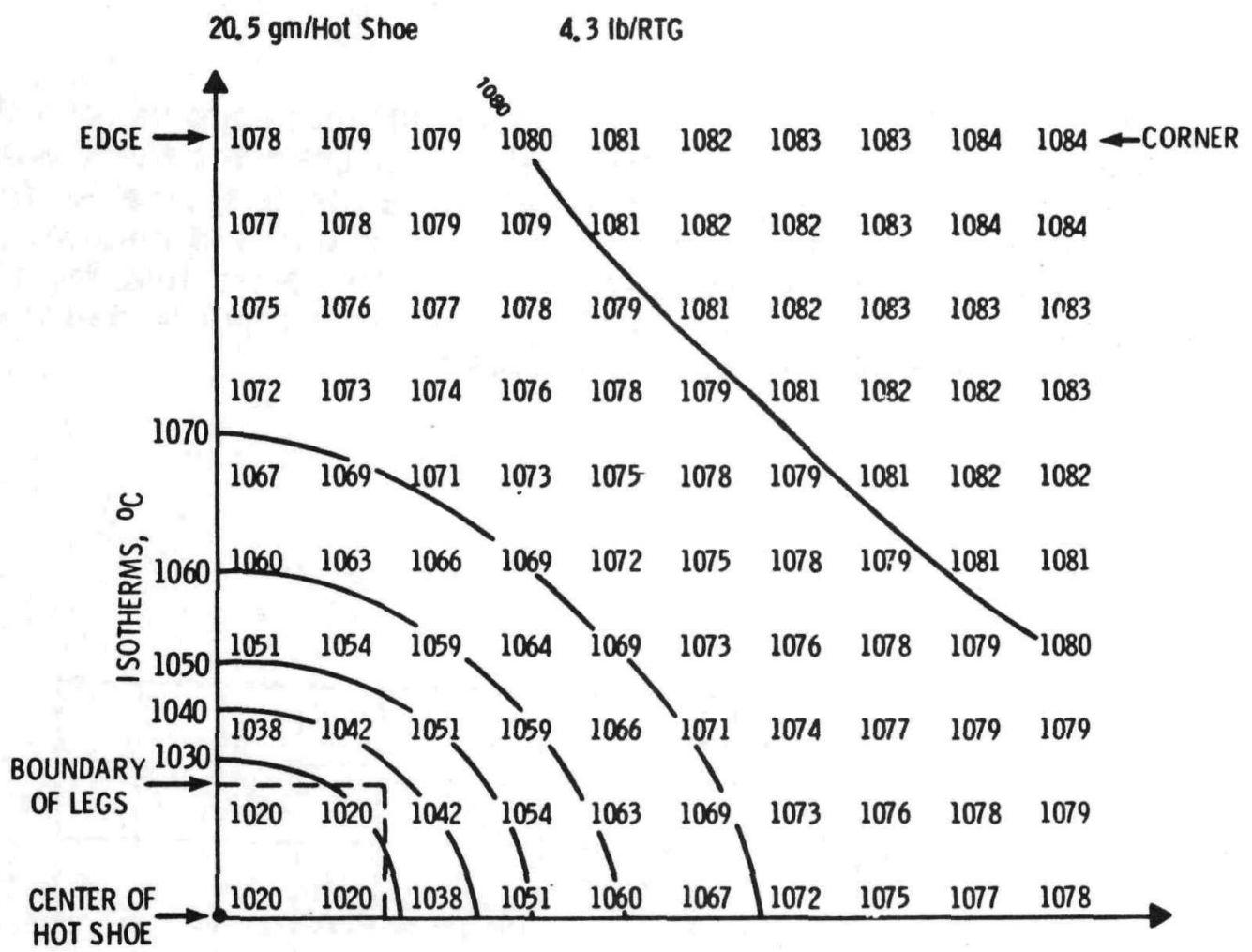

Figure 16. Temperature Distribution $\left({ }^{\circ} \mathrm{C}\right)$ for Uniform Hot-Shoe

The inefficiency of a uniform thickness is shown by the isotherms, which indicate steep gradients near the center of the hot shoe and very little temperature variation near the outside. Clearly, it would be beneficial to remove material from the outer regions and add it to the inner. Such a graded-thickness design is illustrated in Figure 17, which is the result of an iterative optimization. 
The dashed line shows the optimum-thickness profile, and the solid steps represent the analytical model used for the thermal analysis. This thickness profile yields a heat source surface temperature of $1100^{\circ} \mathrm{C}$, and the resultant temperature map for the hot-shoe quadrant is depicted in Figure 18. Note that for this optimized design, the corner of the hot shoe is only $4^{\circ} \mathrm{C}$ below the temperature of the heat source surface.

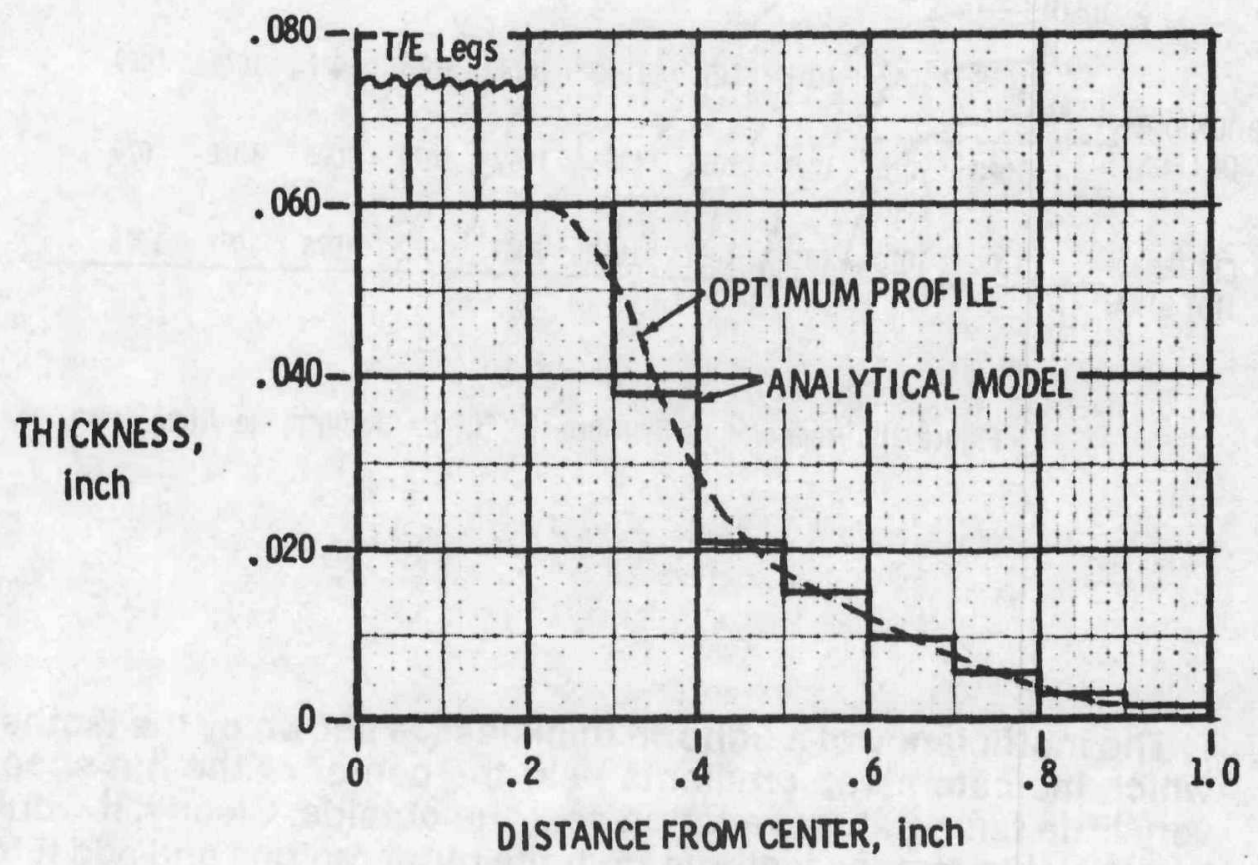

Figure 17. Optimum Hot-Shoe Profile for $1000^{\circ} \mathrm{C}$ Hot Junction and $1100^{\circ} \mathrm{C}$ Heat Source Surface 
Comparison of the isotherms in Figures 16 and 18 shows that the tapered design is much more efficient, producing uniform temperature gradients everywhere except near the corner of the hot shoe. Indeed, this design weighs only 9.6 grams per hot shoe or $\mathbf{2 . 0}$ Ibs per RTG, which is $53 \%$ lighter than the uniform-thickness hot shoe.

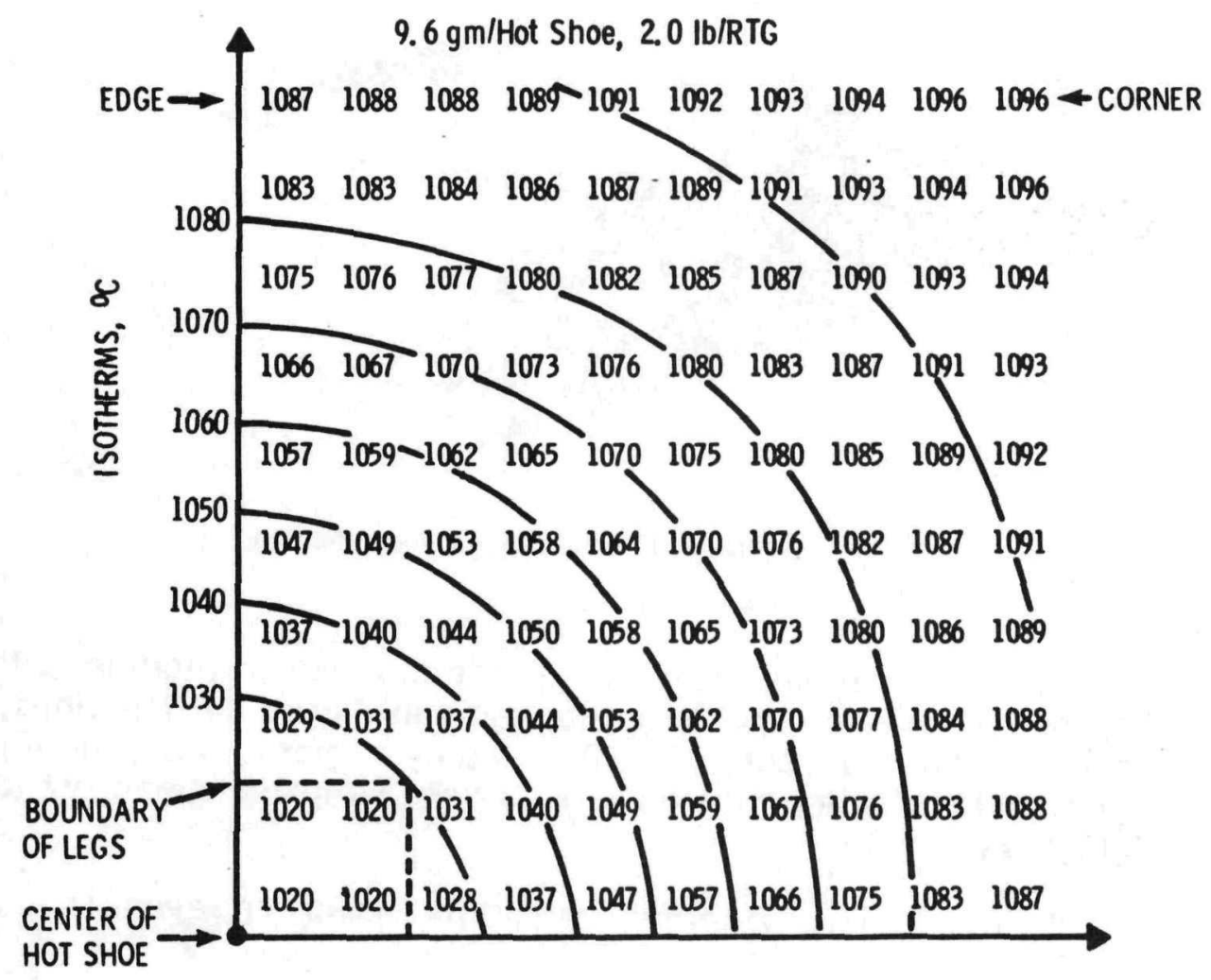

Figure 18. Temperature Distribution $\left({ }^{\circ} \mathrm{C}\right)$ for Tapered Hot-Shoe 
There had been some concern about the fabricability of the optimized hot-shoe profile, and about the dimensional stability of the 0.002 "-thick edges. To allay these concerns, Fairchild machined the molybdenum hot shoe shown in Figure 19, by using a tapecontrolled milling machine. This technique for producing the desired curved tapers appears feasible, and the razor-thin edges showed more-than-adequate dimensional stability.

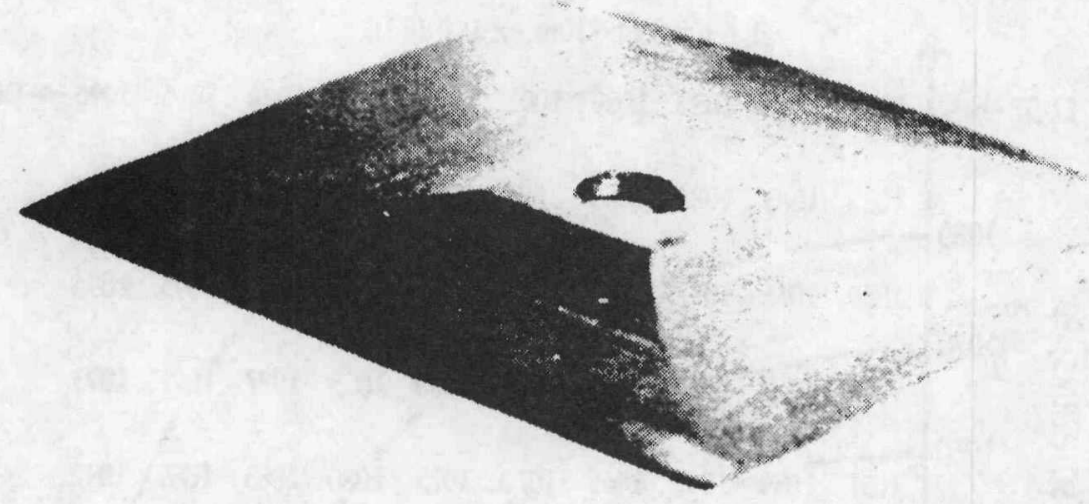

Figure 19. Machined Molybdenum Hot Shoe

A cross-sectional view of the thermoelectric module with the optimum hot shoe profile is displayed in Figure 20 . The figure also shows three layers of 0.012" quartz yarn surrounding the thermoelectric-leg assembly. This yarn wrap had been omitted in previous figures.

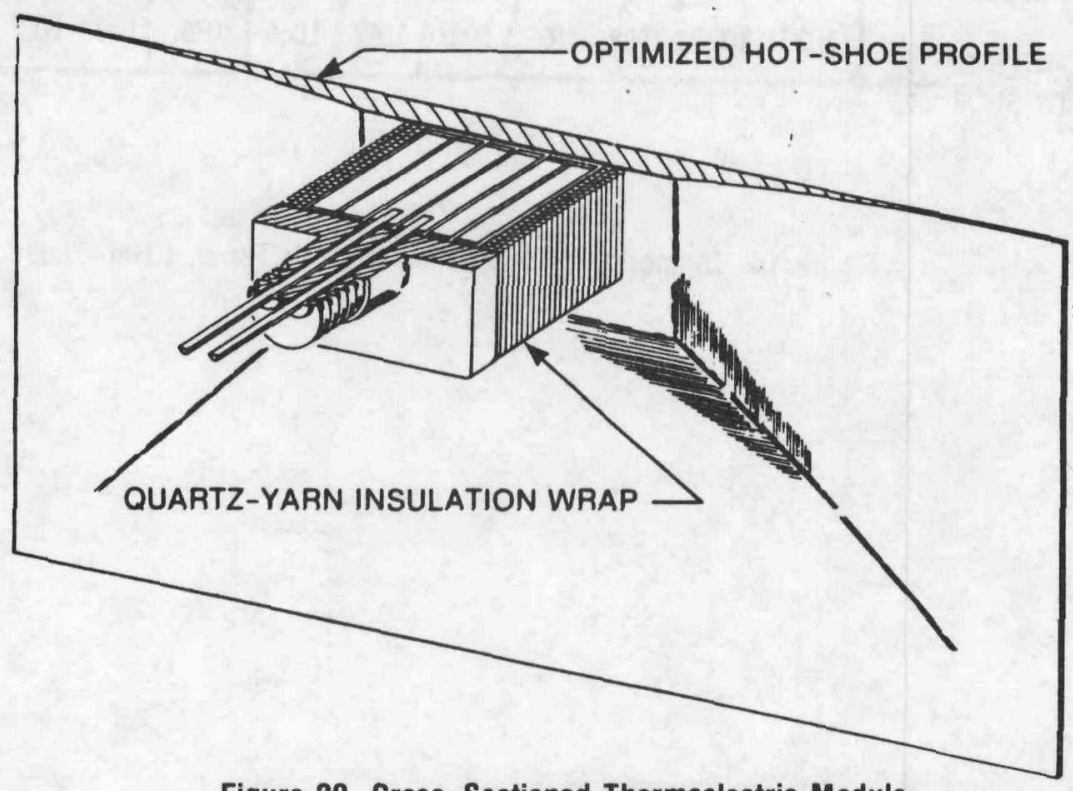

Figure 20. Cross-Sectioned Thermoelectric Module 
An optional additional feature, not shown in previous or subsequent figures, is illustrated by the close-up view of the thermoelectric module shown in Figure 21. This is the addition of very thin diagnostic thermocouples for in-situ measurement of the module's hot-shoe and cold-shoe temperatures. The hot-shoe thermocouple leads are covered by the quartz yarn insulation wrap, and the sheathed leads for both thermocouples emerge through the cold-shoe stud. This avoids the need for additional penetrations through the thermal insulation or through the generator housing, and minimizes the danger of internal shorts.

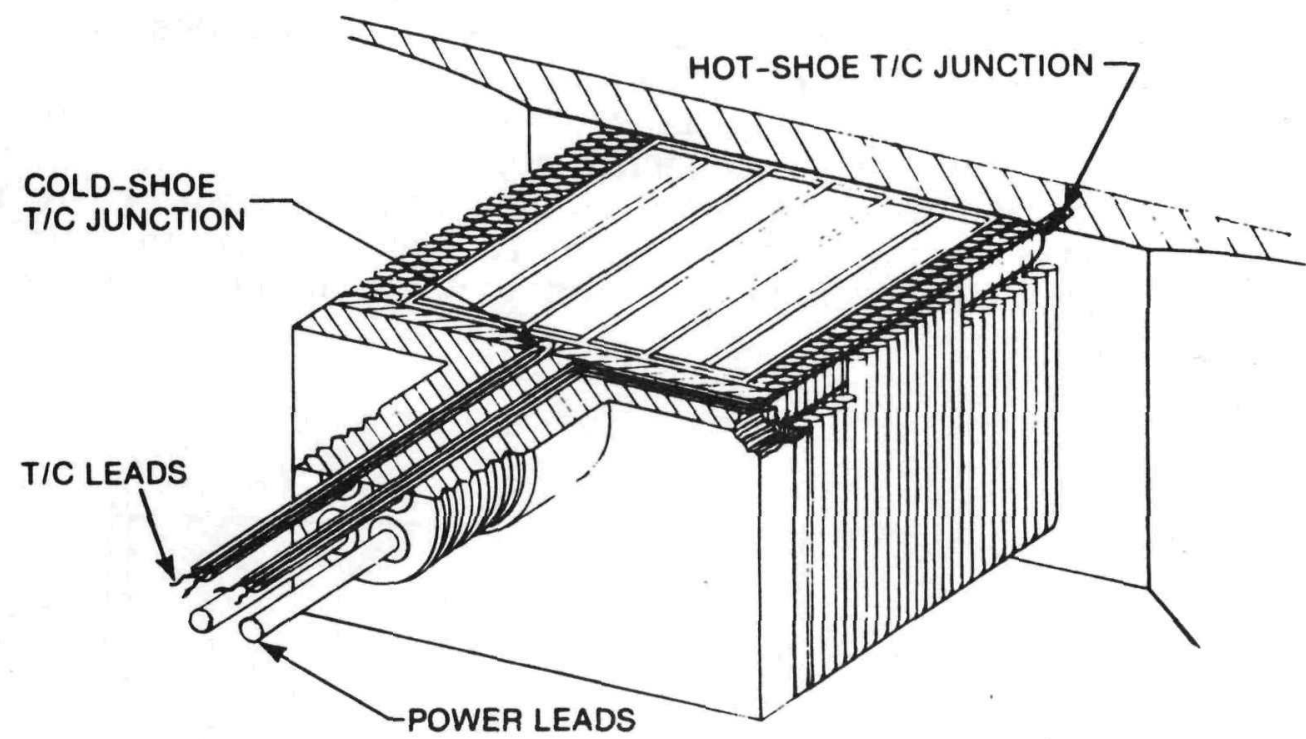

Figure 21. Built-In Thermocouples To Monitor Hot- and Cold-Shoe Temperatures

The ability to instrument each multicouple would be a very desirable feature for development testing, and would also be quite useful for flight hardware. It would make it possible to monitor the hot- and cold-junction temperatures of each thermoelectric module in the assembled generator, and would quickly identify any deficient bonds or excessive contact resistance between the cold shoe and the generator housing. 


\section{MITG HOUSING AND RADIATOR FINS}

The thermoelectric modules described in Sections 4 through 6 are mounted on the inside of the thermally-insulated generator wall. The thermal insulation system is discussed in Section 8 , and the techniques for installing the insulation and the thermoelectric modules in the housing are described in Section 9.

The MITG housing has a square cross-section, to match the almost-square heat source stack. To achieve the desired cold-junction temperature, fins must be added to provide additional radiator area. The current-generation GPHS/RTGs have eight fins, which results in considerable mutual blockage and a relatively high radiator weight. The MITG design uses only four fins, giving each a relatively unobstructed view to space.

The four radiator fins could be located either as axial ribs, along the mid-line of the generator housing sides, or as diagonal fins, connected to the corners of the generator housing. These two options are compared in Figure 22, which shows the effect of fin length on the average view factor to space of the housing and of the fins.

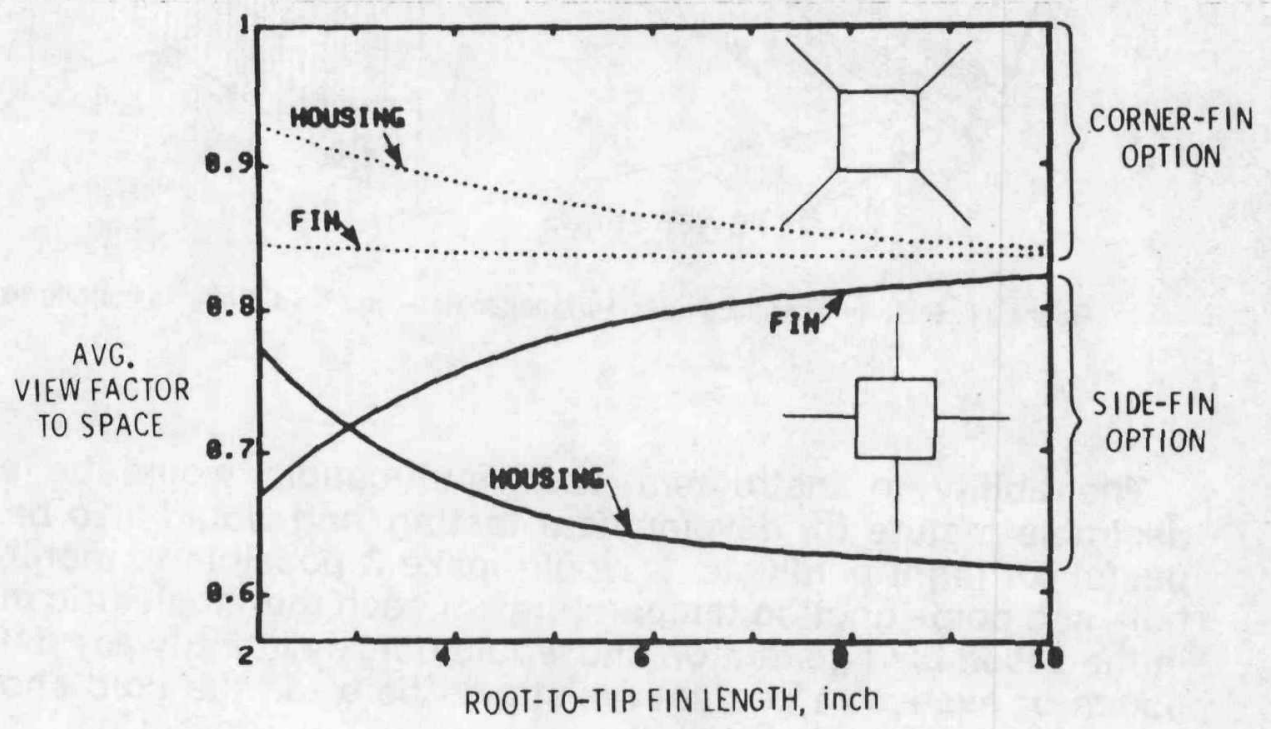

Figure 22. Comparison of Radiator Fin Options 
The view factors shown are all considerably higher than what would be possible with eight fins. As can be seen, the corner fins are much more effective than the side fins, particularly because the housing has a much better view of space with the corner-fin arrangement. The housing, of course, is itself an important part of the radiator.

The superiority of the corner-fin arrangement is confirmed in Figure 23, which shows the required fin dimensions for the two options (for the desired $300^{\circ} \mathrm{C}$ cold-junction temperature). The fins are assumed to be trapezoidal, with an emissivity of 0.90 , a fin tip thickness of 15 mils, and with the fin root thickness and fin length optimized to minimize their weight. As shown, the corner fins are shorter, thinner, and weigh $37 \%$ less than the side fins.

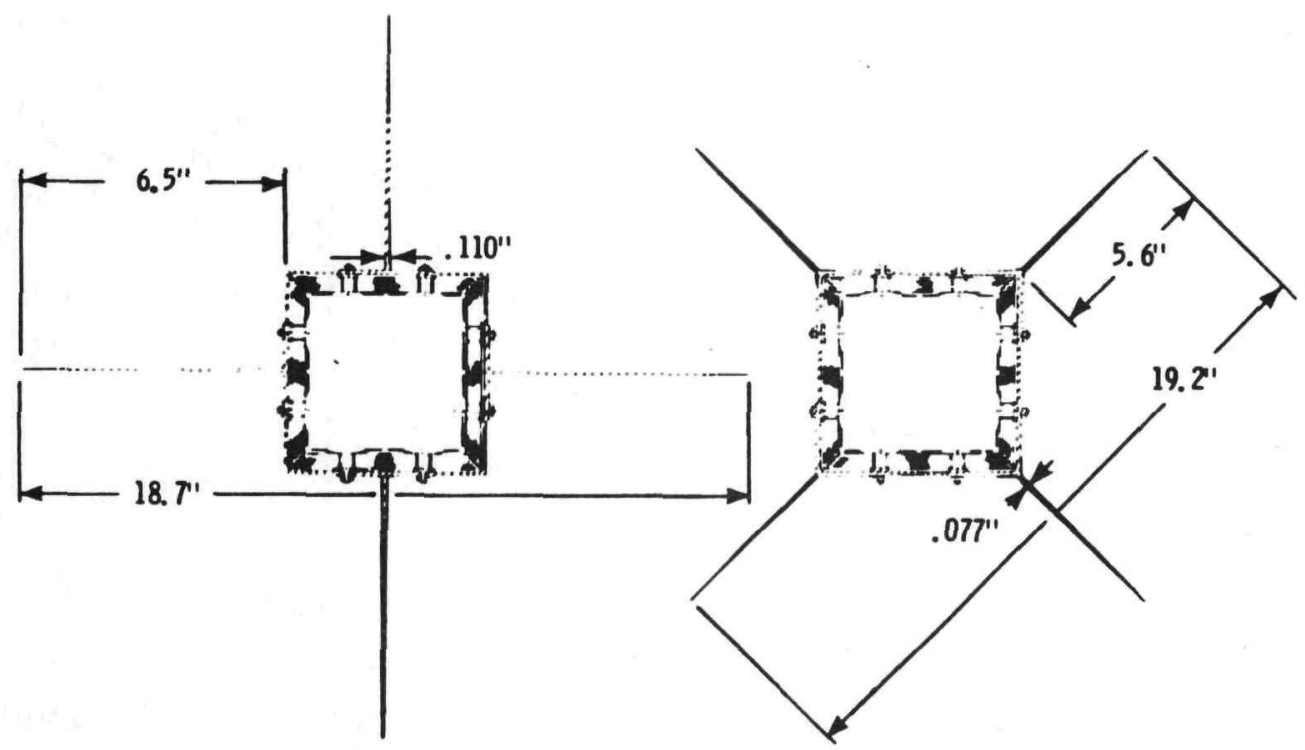

Figure 23. Comparison of Side and Corner Fins 
Figure 24 shows a section of the generator housing with truncated corner fins. The figure shows an auxiliary coolant duct near the base on each fin. These ducts carry water for cooling the generator housing while the spacecraft is inside the shuttle, but are not needed during operation in open space.

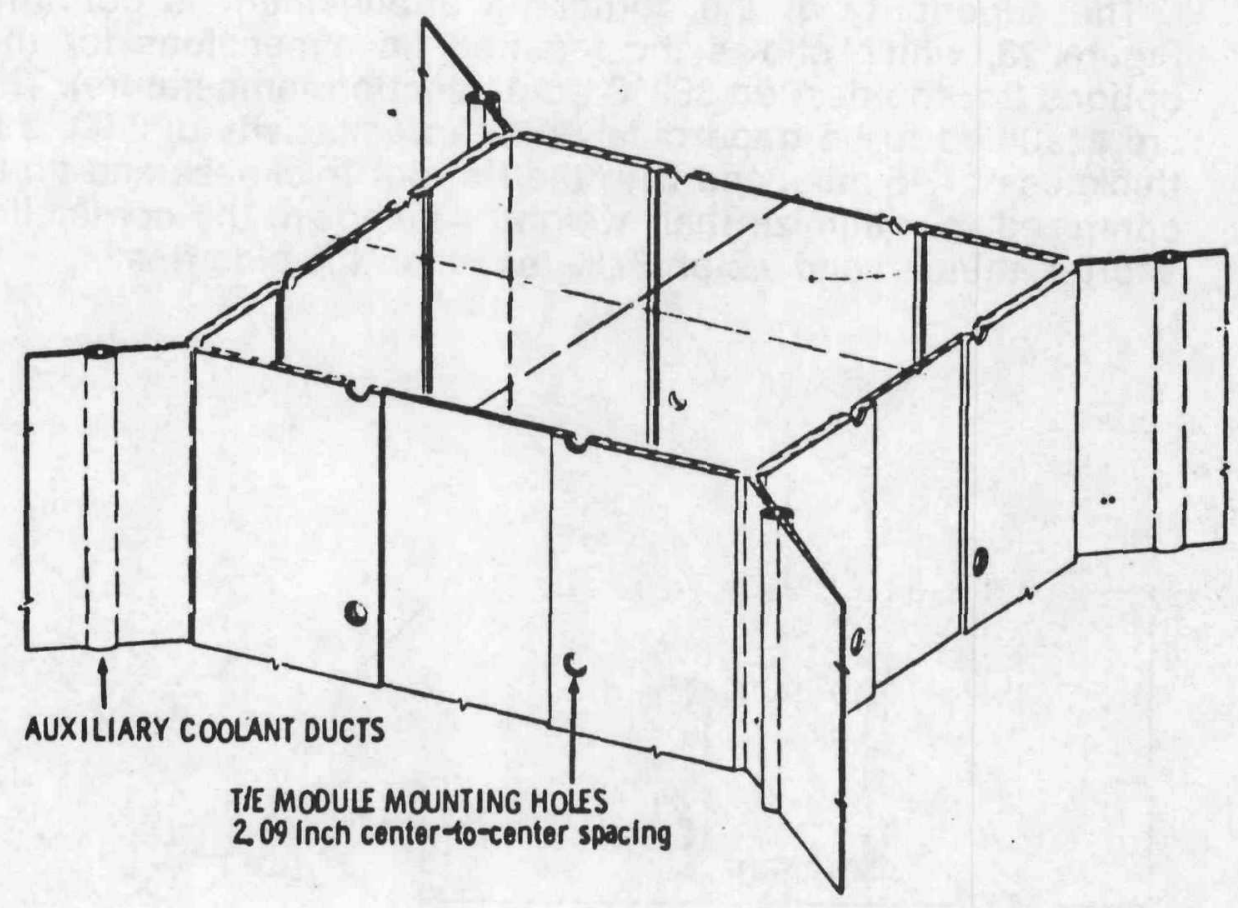

Figure 24. Generator Housing Section with Corner Fins

The housing sides have noles for mounting the thermoelectric modules, with a 2.09" center-to-center spacing between holes. Each side wall has a thickness of $1 / 16$ " near its center, and $1 / 8$ " near the corners, to accomodate heat flow from the thermoelectric modules to the radiator fins without excessive temperature drops. These thickened edge regions are fortuitous, because they strengthen and stiffen the generator housing, which plays the structural role of a cantilevered box beam. 


\section{THERMAL INSULATION}

RTGs require thermal insulation to minimize the heat flow from the thermoelectric hot shoe to the generator housing. The thermal insulation used in the MITG consists of many layers of very thin metal foils, separated by spacers. This type of insulation has been used extensively, and has proven highly effective in reducing radiative heat transfer in a vacuum environment. Multifoil insulation had also been used in the MHW and GPHS/RTGs. These generators used 60 layers of 0.3 mil molybdenum, with alternating layers of open-weave quartz cloth.

In the MITG design the quartz cloth spacers have been replaced by a low-density coating of zirconia particles. This reduces the thickness of the 60-foil package from 0.7 to 0.3 inch, and decreases the insulation weight by more than $50 \%$. The weight reduction is itself important, because the insulation package is an important component of the generator weight; and the thickness reduction results in additional weight saving, by shrinking the size of the required generator housing and allowing the use of shorter thermoelectric legs. Multifoil insulation with zirconia particle spacers has been in use by Thermoelectron for a number of years, and has shown excellent performance stability [12]. Some multifoil assemblies of this type have also been vibration-tested, to confirm that the spacer particles will stay in place during launch vibration. 
Figure 25 shows a typical temperature distribution for 60 foils operating between $1000^{\circ} \mathrm{C}$ and $300^{\circ} \mathrm{C}$. While previous RTGs have used molybdenum foils, the use of other materials is under consideration for the MITG. The figure illustrates a possible combination showing the use of niobium for the hottest 32 layers, followed by $\mathbf{2 5}$ layers of titanium or vanadium, with the three coolest layers consisting of aluminum. The advantage of these materials is not only the potential weight reduction of the foil package, but also their better thermal expansion match to the aluminum housing.

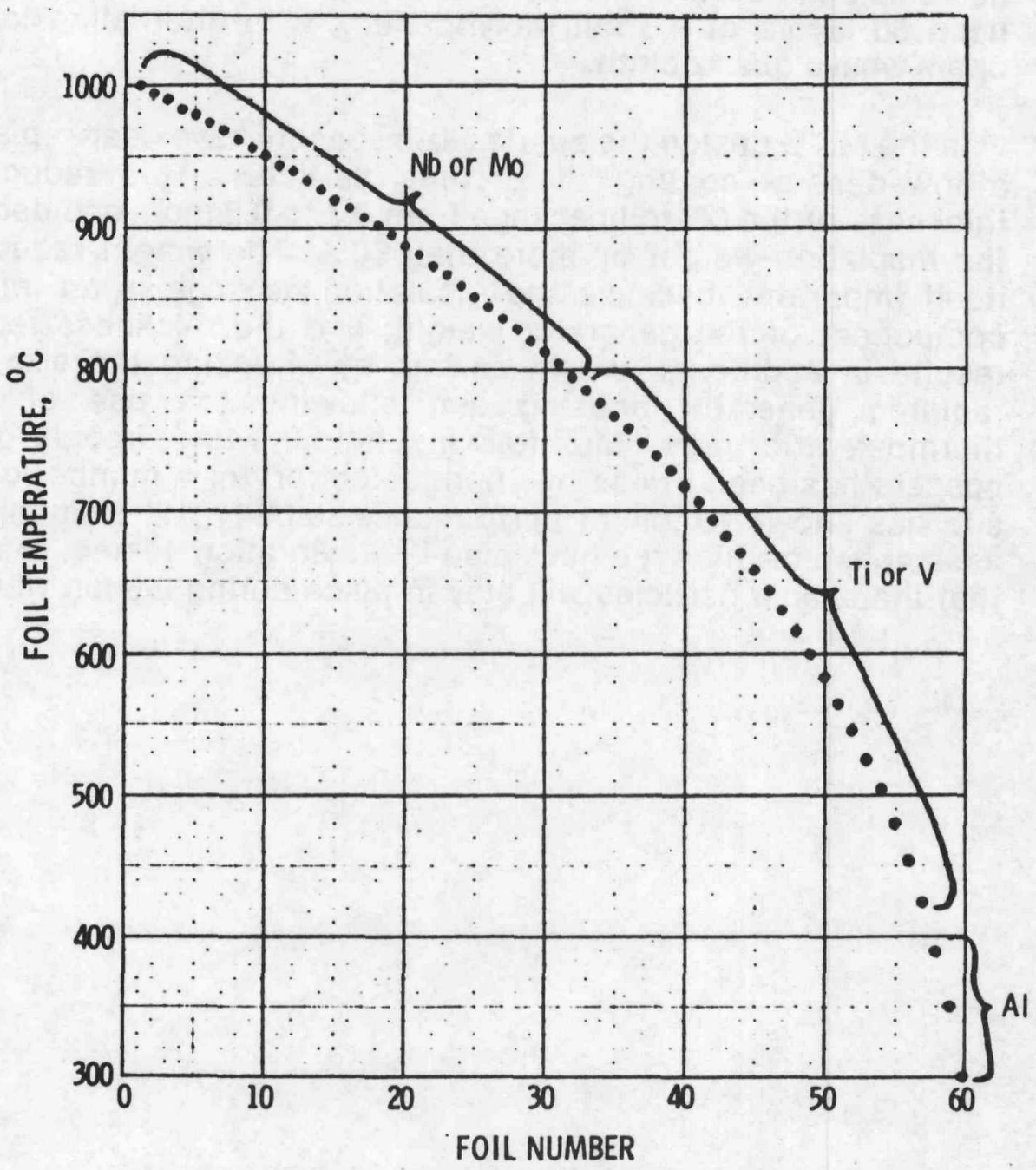

Figure 25. Multifoil Insulation Temperature Distribution 
The multifoil insulation in the generator is supported by the thermoelectric modules, which in turn are mounted on the generator housing. The support provided by the thermoelectric legs is quite rigid, though there is some compliance provided by the three layers of quartz yarn wrap. While the SiGe legs are relatively strong, excessive shear loads must be avoided. The effect of differences in thermal expansion between the hot and cold foils and the aluminum housing must therefore be examined.

Figure 26 shows the thermal expansion of the different foils relative to the housing. The circled points denote the material choices suggested in Figure 25. Relative expansions are given in mils per inch, but since the thermoelectric modules have a center to center spacing of 2" and the midpoint between two modules may be considered as a neutral point, the vertical scale also represents the expansion mismatch in mils at the interface between the foils and the thermoelectric module wrap.

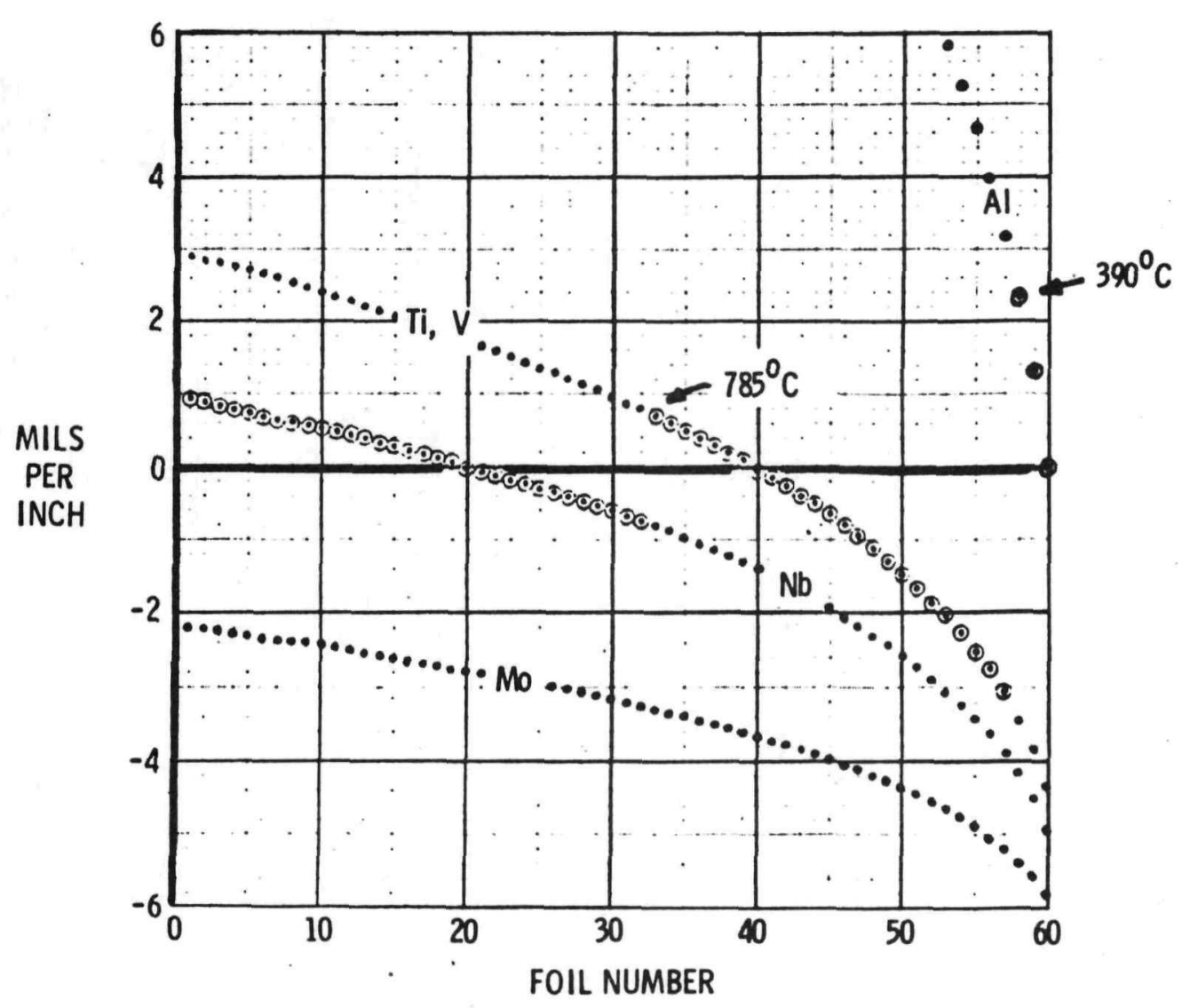

Figure 26. Thermal Exansion of Foils Relative To Housing 
As can be seen, the mismatch for the proposed materials is less than three mils, which can be accommodated by the compliance of the foils and the quartz yarn wrap. Thus, it is feasible to use small patches of foil with $2 \times 2$ thermoelectric module penetrations. But for larger foil areas, expansion joints will probably be needed to avoid excessive shear loads on the thermoelectric modules. The design of these expansion joints is critical, because heat loss through poorly designed joints can be much larger than losses through the unbroken areas of the foil package.

Figure 27 shows one possible foil arrangement, using stepped joints between 4-hole multifoil sections. This arrangement, which has been used in the past, would be relatively simple to apply, but not as effective thermally as some of the other options considered.

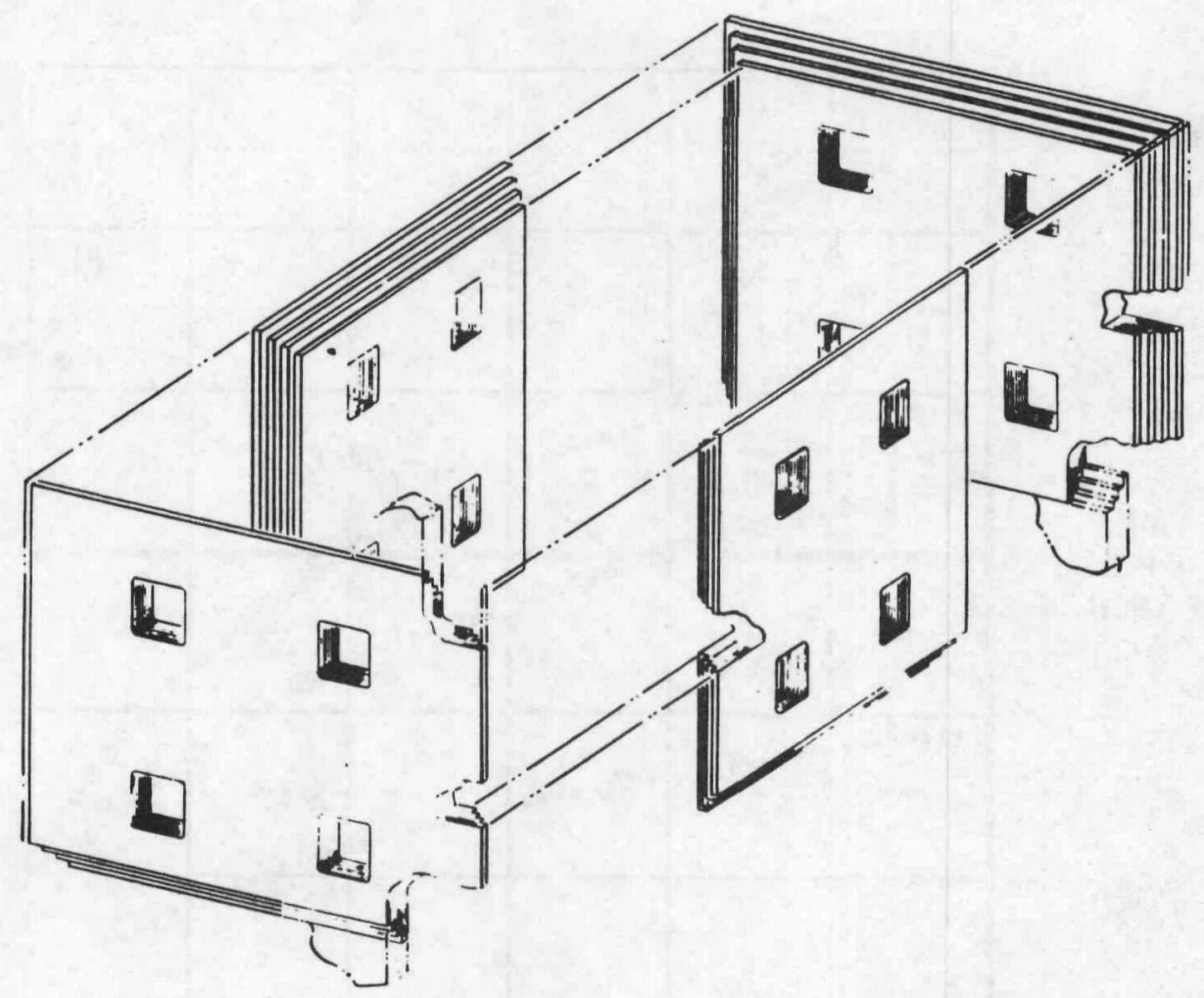

Figure 27. Multifoil Arrangement with Stepped Joints 
Figure 28 illustrates a pleated foil arrangement. With this arrangement, the entire foil package for each side of the generator could be assembled as a unit, with step joints used at the edges of the generator. The pleats would provide enough axial compliance to compensate for differential thermal expansion in that direction, and would also provide lateral stiffening to resist g-loads normal to the plane of the foil package. When the pleated foil package of this type is inserted into the snug-fitting generator housing, a rather stable structure results.

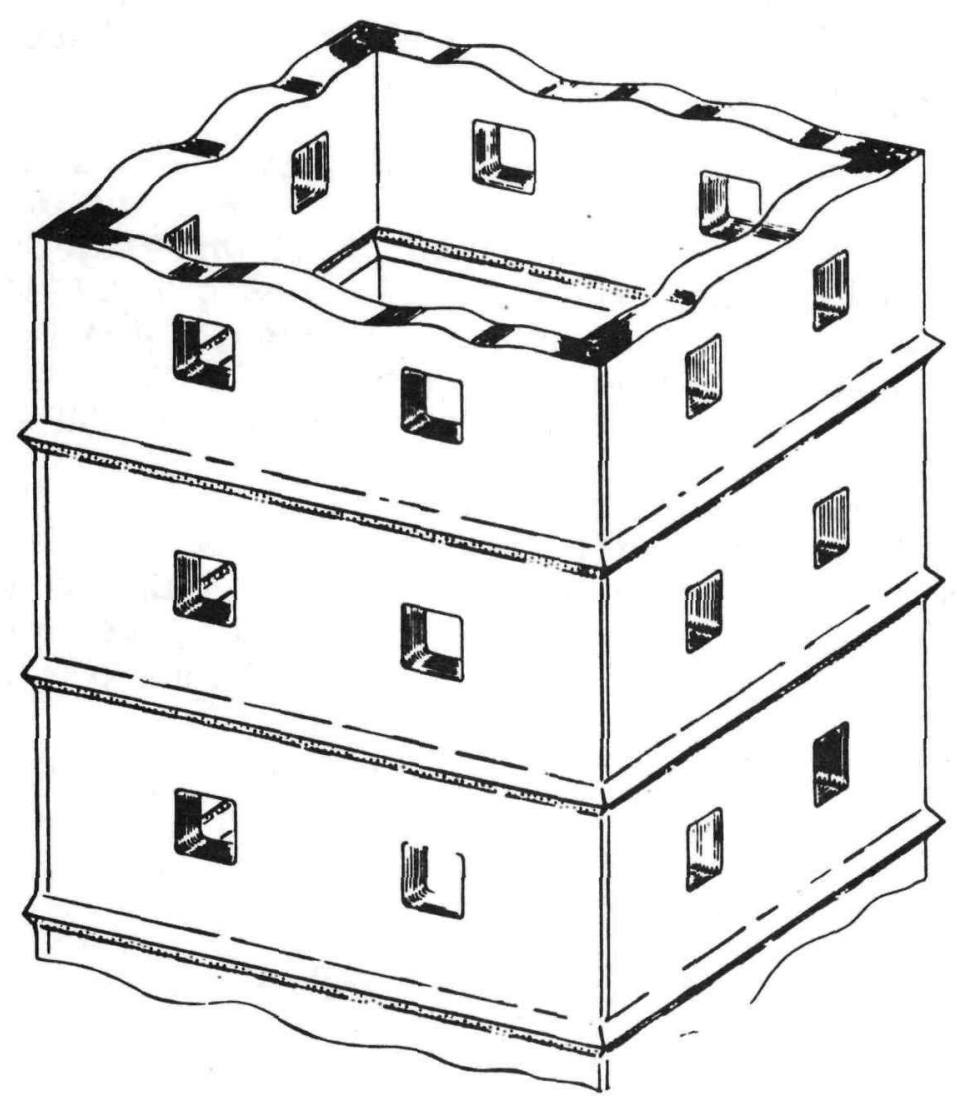

Figure 28. Pleated Multifoil Insulation Assembly, Showing Axial Compliance and Lateral Stiffness Provided by Pleats 
A third option, illustrated in Figure 29, would result in the lowest possible heat losses at the joints. This option, which was suggested by Thermoelectron, makes use of interleaved foils at all edges and at other expansion joints. The foil is applied in patches, each containing $2 \times 2$ thermoelectric module penetrations. As shown by the two cut-away sections, each foil patch is oversized to provide overlaps with neighboring foils axially, and is provided with edge flaps to provide overlaps with foils around the corner. For this design, the foil packages must be laid up one patch at a time to provide the desired interleaving. While this assembly procedure is time-consuming, it appears to be quite feasible, as explained in Section 9. Therefore, it is currently the preferred approach, because it minimizes parasitic heat losses.

The figure also shows a dashed square around each of the foil windows. These squares represent the outer edges of molybdenumopacified $\mathrm{SiO}_{2}$ paper washers, which alternate with the metal foils. Such washers have been successfully used by General Atomics in RTG development tests. In the MITG design, they help to maintain the desired 5-mil spacing between the 0.3-mil molybdenum foils, particularly during the insertion of the thermoelectric modules.

It may also be desirable to make the windows slightly over-sized in the metal foils, but not in the $\mathrm{SiO}_{2}$ paper washers. This would allow some movement by the metal foils to accommodate differential thermal expansion, while the snug-fitting paper washers prevent interface gaps through which radiation streaming could occur. 


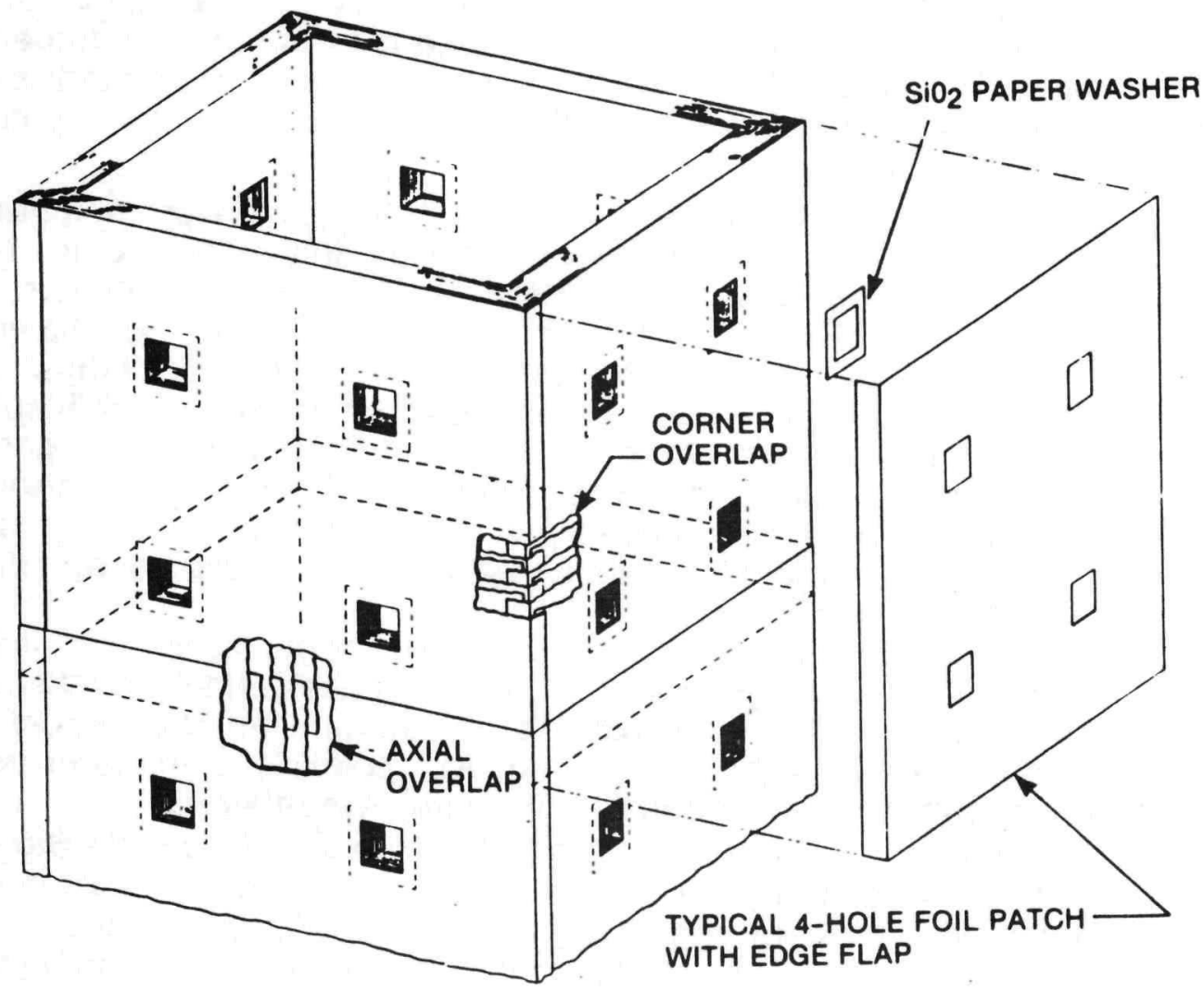

Figure 29. Interleaved Multifoil Assembly 


\section{CONVERTER ASSEMBLY PROCEDURE}

This section describes the assembly and installation of the interleaved multifoil insulation in the housing of a typical 12-slice MITG, and the subsequent installation of the thermoelectric modules. These installation procedures are illustrated by the sequential Steps 1 through $\mathbf{8}$ shown in the cross-sectional plan view shown in Figure 30.

Step 1 shows a dummy module, with a square stud matching the dimensions of the yarn-wrapped leg assembly and the cold shoe of the thermoelectric module. Also depicted is a small screw for attaching the dummy module to the central mandrel, as shown.

The mandrel has a square cross-section, and its outer dimensions match the desired inside dimensions of the multifoil insulation package. It extends over the full height of the generator, and contains 96 threaded holes at the desired thermoelectric module locations. As shown, the mandrel has eight shallow axial grooves, which accommodate the thin flanges at the inner ends of the dummy modules.

After all 96 dummy modules have been mounted on the mandrel, it is inserted into the generator housing to make certain that there is no hang-up during insertion, and that the dummy module centers are in proper registry with the housing wall holes. The mandrel is then withdrawn, for application of the multifoil insulation.

The exploded portion of Step 2 shows two of the square SiO 2 paper washers (depicted earlier in Figure 29); and a section of a 4-hole foil patch with its bent edge flap, ready for mounting on the dummycovered mandrel. These foil patches are prepared by clamping a large number of foil layers between two rigid plates with four square windows, and electric-discharge-machining all four windows through the foll package simultaneously. Thermoelectron has demonstrated that this can be done very cleanly, without welding the edges of the molybdenum foil windows. After this, each individual foil patch is bent to form the flap for the desired edge overlap.

The foil patches are deposited on the mandrel, with the four holes of each patch slipped over the square studs of four dummy modules. They are applied individually, one complete circumferential and axial layer at a time, to give the interleaving at the axial and edge overlaps illustrated in Figure 29. Deposition of the foil layers alternates with installation of the $\mathrm{SiO}_{2}$ paper washers. The assembled view shown in Step 2 of Figure 30 represents the mandrel, covered with a complete 60 -layer foil assembly, ready for insertion into the MITG housing.

In Step 3, the foil-covered mandrel is inside the generator housing. The housing wall has 96 threaded holes, which line up with the small screws that attach the dummy modules to the mandrel. The housing wall holes are large enough to permit removal of the small mandrel screws from the outside of the MITG housing, as illustrated. 
(8)

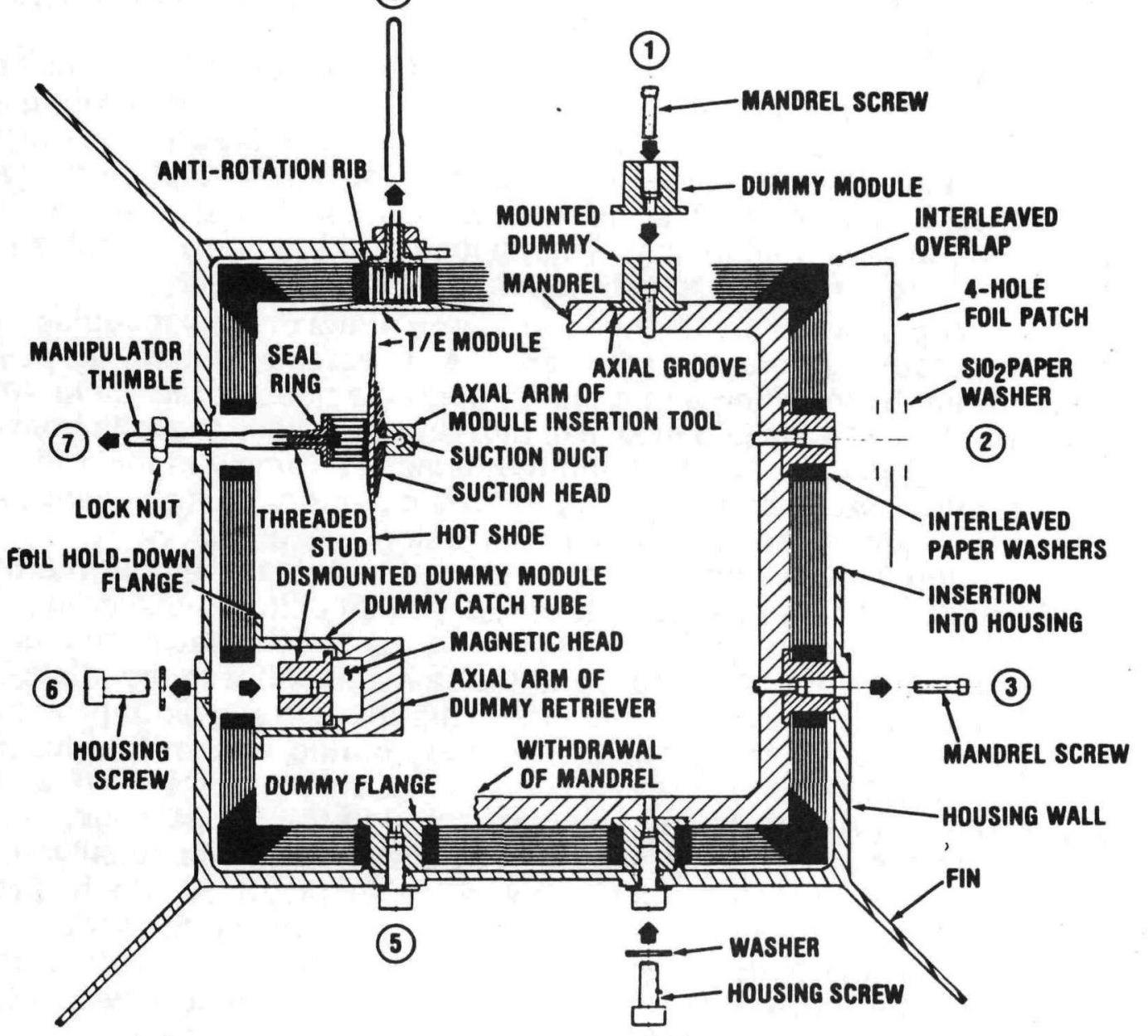

(4)

Figure 30. Converter Assembly Procedure 
The small-diameter mandrel screw removed in Step 3 is replaced in Step 4 by a larger-diameter screw and washer, which fastens the dummy module to the inside of the housing wall. This screw replacement is completed for one dummy at a time, to make certain that proper registry between the multifoll penetrations and the housing wall holes is maintained.

After all 96 dummy modules have been fastened to the inside of the housing wall, the inner mandrel is free to be axially withdrawn from the insulated housing. During this withdrawal, the dummy modules serve as lateral guides for the grooved mandrel. As shown in Step 5, this leaves the multifoil package anchored to the housing wall, in proper alignment. The flanges of the dummy modules keep the foils from slipping off their inner ends.

Step 6 illustrates the removal of one of the dummy modules. After the housing screw is removed, the unfastened dummy is pushed inward by inserting a thin tool through the housing wall hole. At the same time, a flanged tube-like tool is used to keep the foils from also moving inward, and to catch the detached dummy module for axial removal. As shown, this step leaves an empty multifoil window, in registry with the corresponding housing wall hole.

Step 7 illustrates the installation (through the empty multi-foil window) of the real thermoelectric module pictured earlier in Figure 20. Note that a small metal $\mathrm{C}$-ring has been placed around the module's cold-shoe stud, and that a manipulator thimble has been attached to the end of that stud. This thimble serves to protect the power leads and thermocouple leads during the module insertion step, and makes possible the manual positioning of the T/E module while its cold shoe and legs pass through the foil package.

As shown in Step 7, the thermoelectric module is initially held at the center of its hot shoe, by a vacuum suction head attached to the axial arm of the module insertion tool. This tool first lines the module up with the housing hole. The alignment can be visually checked from the outside, through the hole. The tool then moves the TIE module outward, until the end of the thimble emerges from the hole. The module is then picked up manually by means of the attached thimble, and is uncoupled from the insertion tool.

Final insertion of the T/E cold shoe and yarn-wrapped leg assembly into the multifoil hole is carried out manually. This provides tactile feedback to tell the operator whether the operation is proceeding smoothly, and allows him to make final adjustments in module position, if necessary.

After the module has been fully inserted, it is locked in place by the external nut, while the T/E module is kept from rotating by the anti-rotation rib on the inside of the generator housing. This is illustrated in Step 8 of Figure 30, which also shows the subsequent removal of the manipulator thimble.

The replacement of each dummy module by a real module, as illustrated in Steps 6 through 8, must of course be completed one modisle at a time, to ensure retention of proper multifoil alignment. 


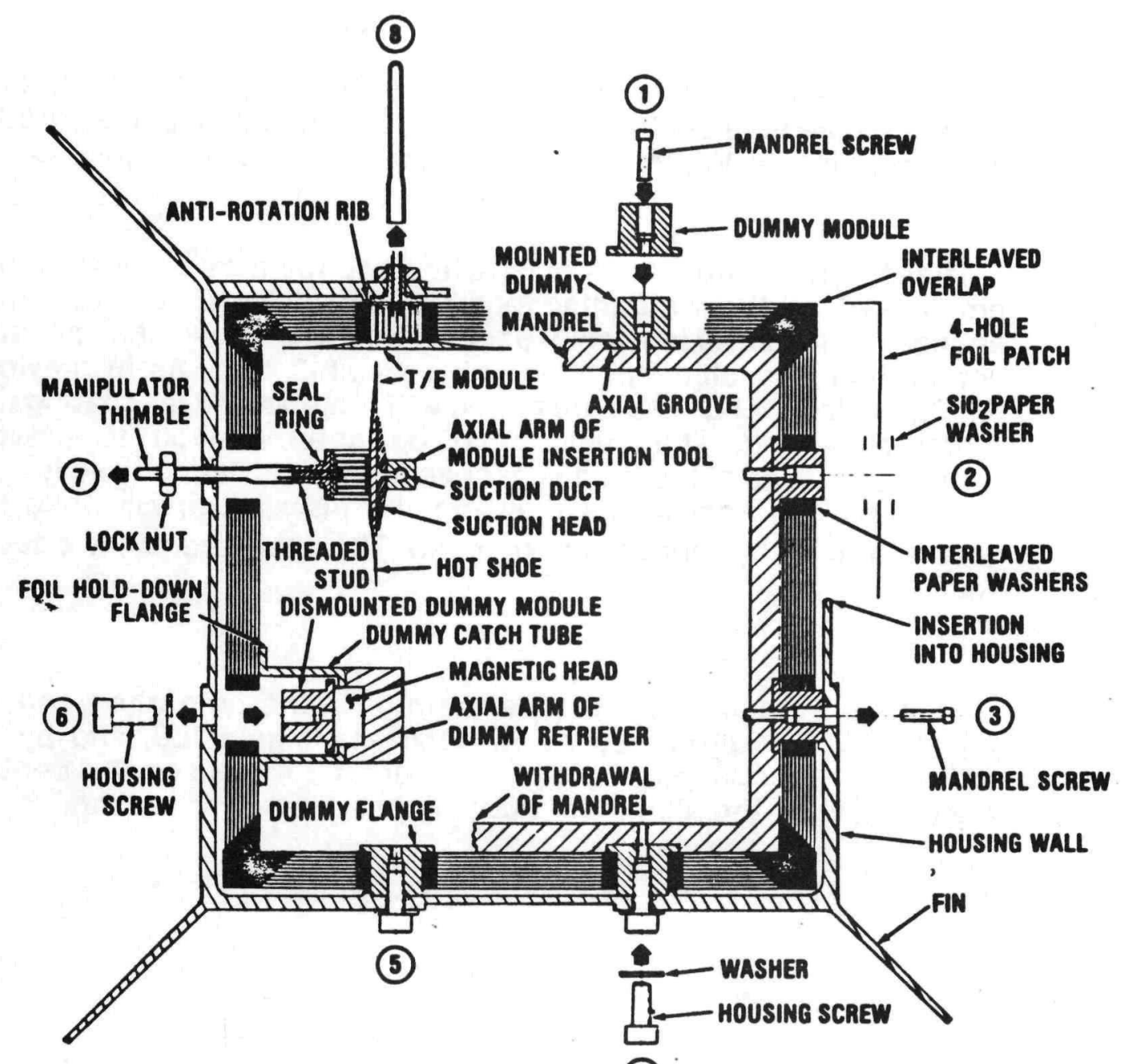

(4)

Figure 30. Converter Assembly Procedure 
A more detailed depiction of the mounting of the thermoelectric module to the insulated wall of the generator housing is presented in exploded view in Figure 31 and in assembled view in Figure 32.

These figures show more clearly how the thermoelectric modules are locked in their mounted position by an external nut which engages the threaded cold-shoe stud, and how the housing penetration is sealed by means of a small C-ring. As in previous RTGs, the Inconel C-rings are plated with gold and silver coatings, to promote sealing. The seals must be good enough to prevent excessive losses of the inert cover gas during pre-launch and launch operations. This cover gas prevents the infusion of air while the generator is in the earth's atmosphere. The seals are not needed in space.

Thermal contact between the thermoelectric cold shoe and the housing wall is promoted by the compliant gold foil, and by the differential thermal expansion which greatly increases the contact pressure when the aluminum housing heats up after fueling. 


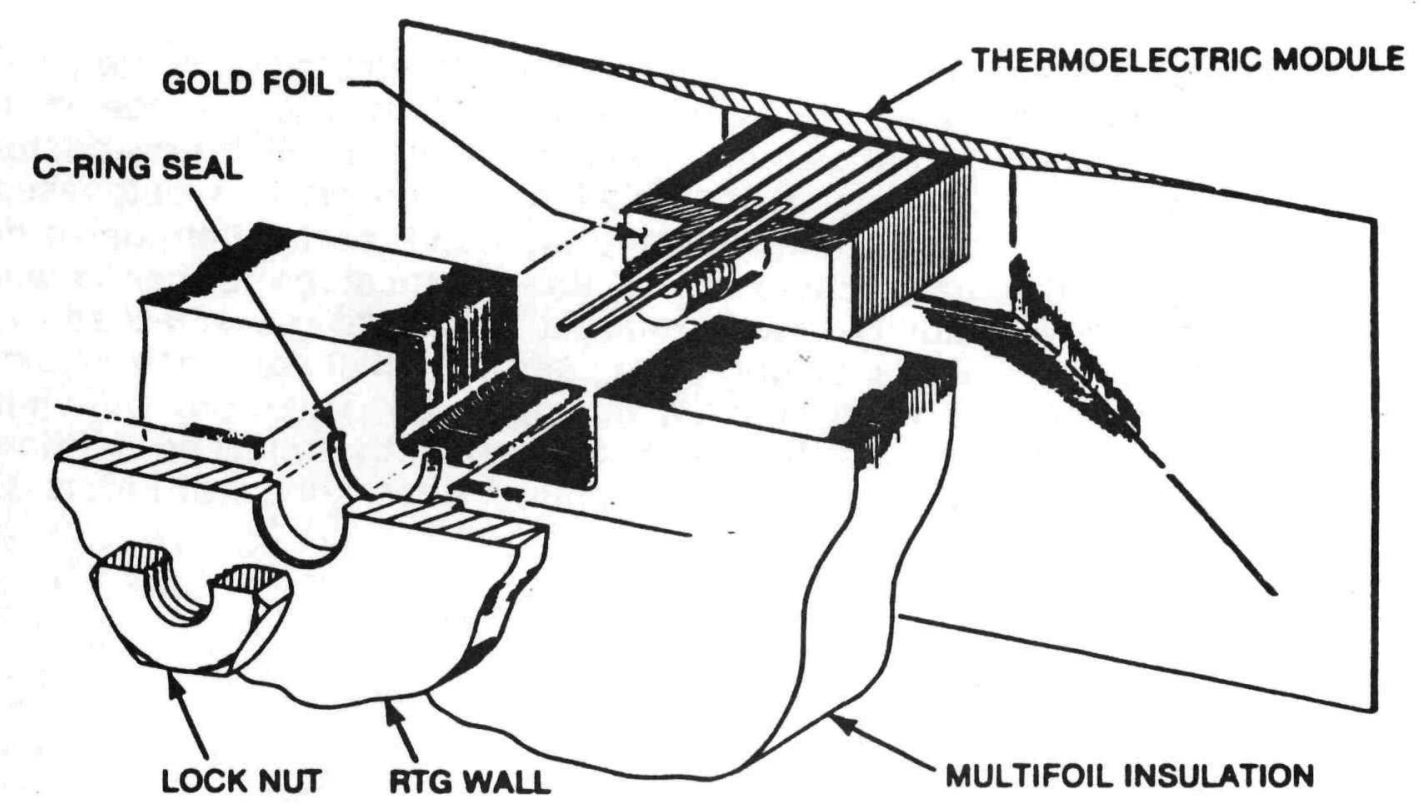

Figure 31. Mounting and Sealing Arrangement (Exploded View)

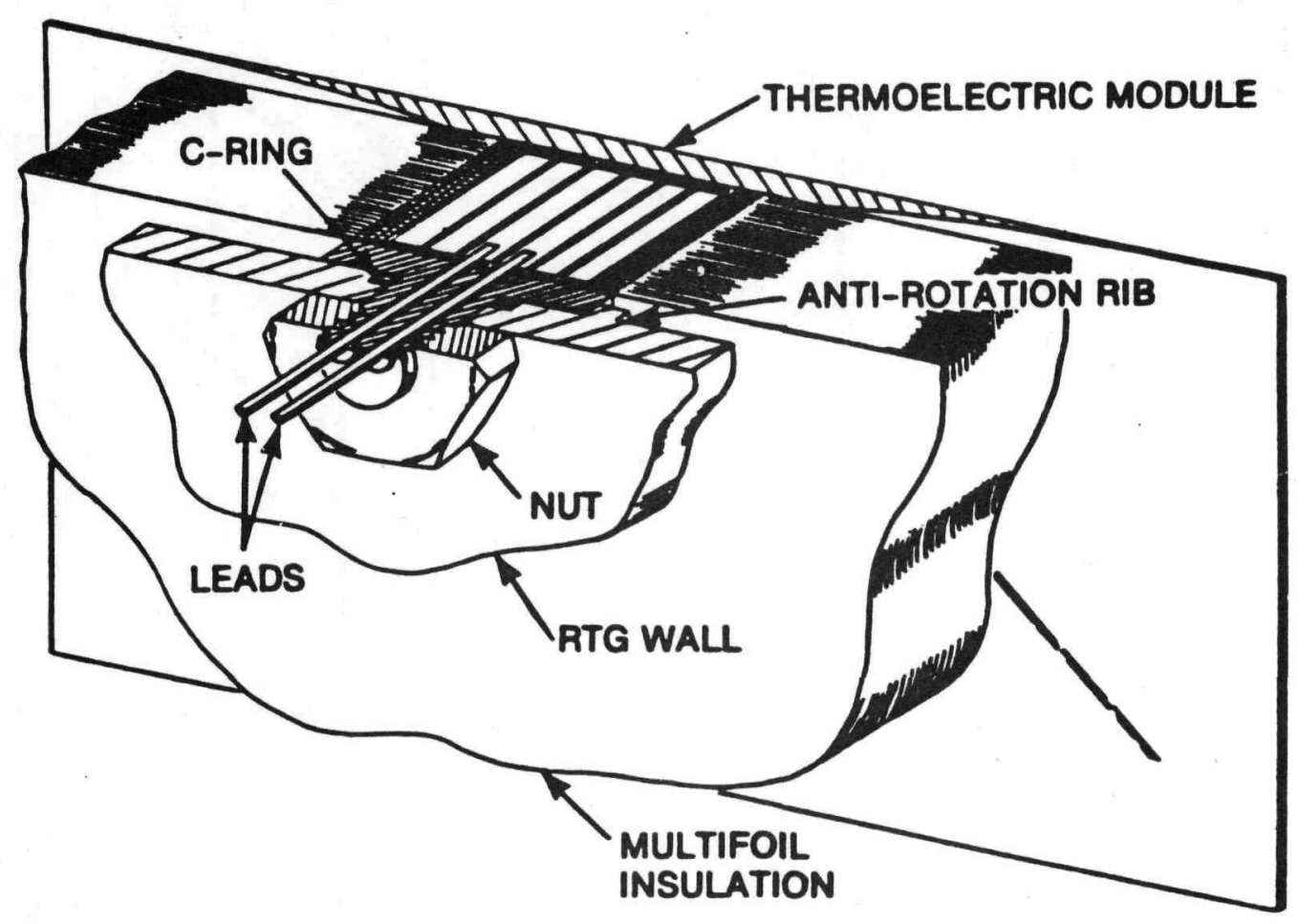

Figure 32. Mounted Thermoelectric Module 
Figure 33 shows a sectioned view of the assembled converter, with all modules mounted on the insulated housing. The thermoelectric hot shoes form a central cavity, ready to receive an electrical heater. By means of this heater, the full-length converter is outgassed, brought up to operating temperature, and each thermoelectric module individually checked for its electrical performance and performance stability. Each module can also be checked for leaktightness of its C-ring seal, and for thermal conductance between its cold shoe and the housing wall. Thus, any deficient module can be identified and subsequently replaced without perturbing any of the others, by reversing Steps 7 and 8 of Figure 30

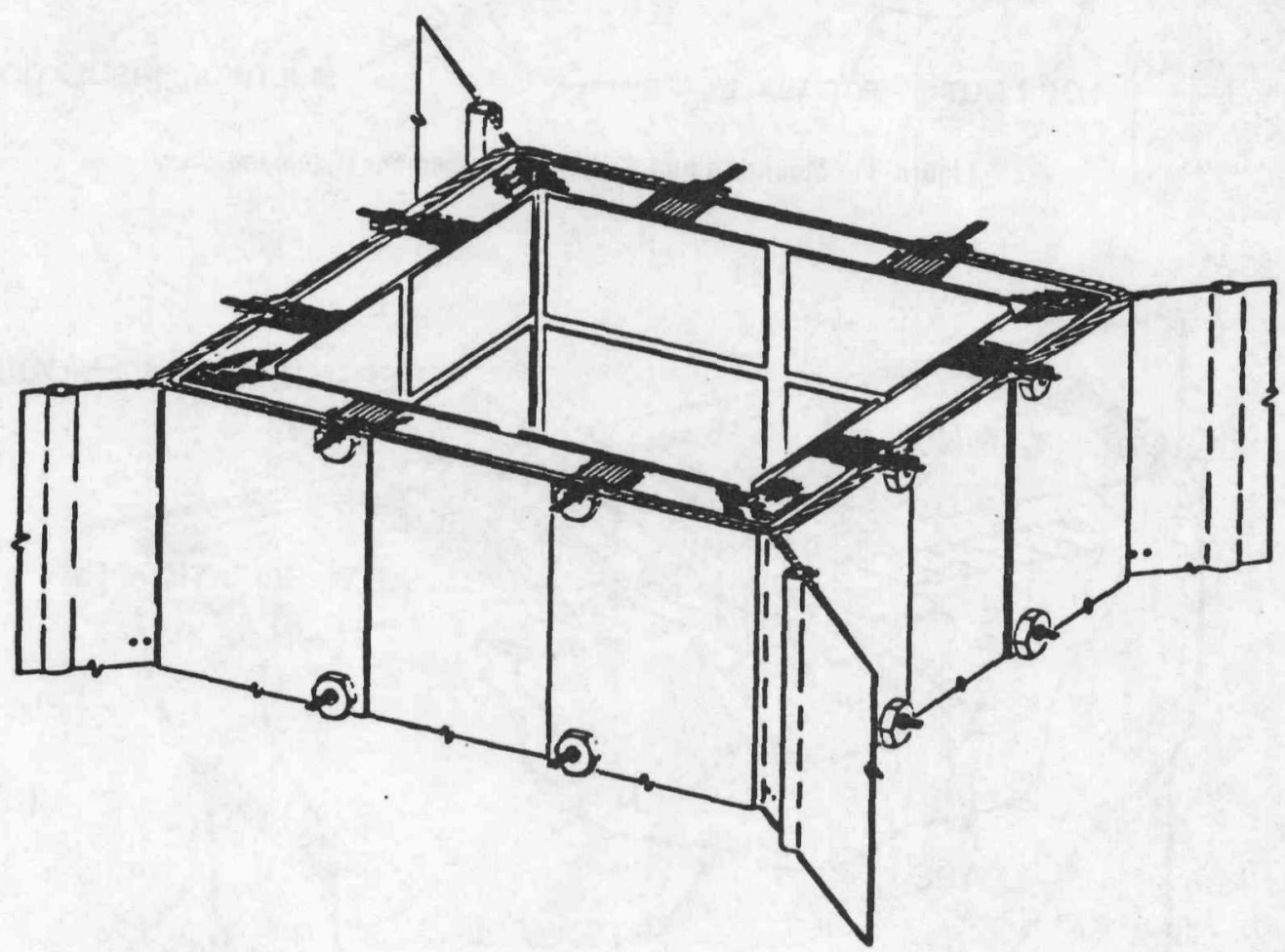

Figure 33. Assembled Converted, Showing Central Heater Cavity Formed by Hot Shoes 


\section{ELECTRICAL CIRCUIT}

Figure 34 shows a typical converter slice with the external electrical circuit added. The figure illustrates the most basic circuit arrangement. The eight thermoelectric modules are connected in series by the horizontal loop circling the generator slice. Relatively small wires and crimped connectors can be used, since the loop carries less than 1 ampere. The vertical wires normally carry no current, except for two identified as the 28-volt busbars, which connect all the generator slices in parallel. The other vertical wires are added only to form a series-parallel network, which makes the circuit highly resistant to open-circuit failures.

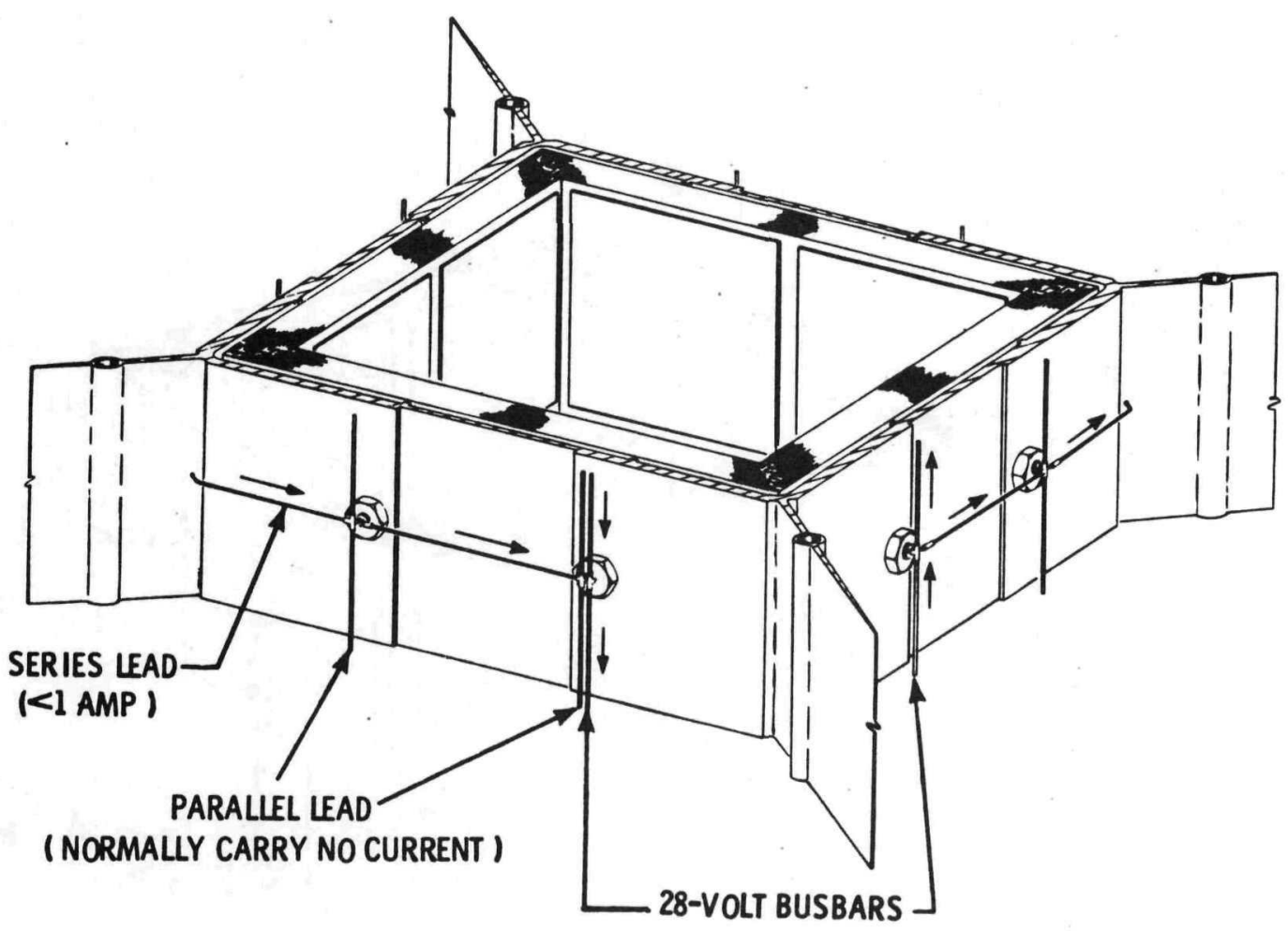

Figure 34. Standard Converter Slice with Basic Circuit Module 
A slightly more complicated circuit option is illustrated in Fig. 35 . For many NASA missions it is important to minimize the generator-induced magnetic field, to avoid interference with scientific instrumentation on the spacecraft. The figure illustrates a design in which the self-induced field is eliminated almost completely. At the end of the current loop which connects the eight T/E modules in series, the current is brought back through a reverse loop, so that the two busbars are located adjacent to each other. This essentially cancels the induced magnetic field.

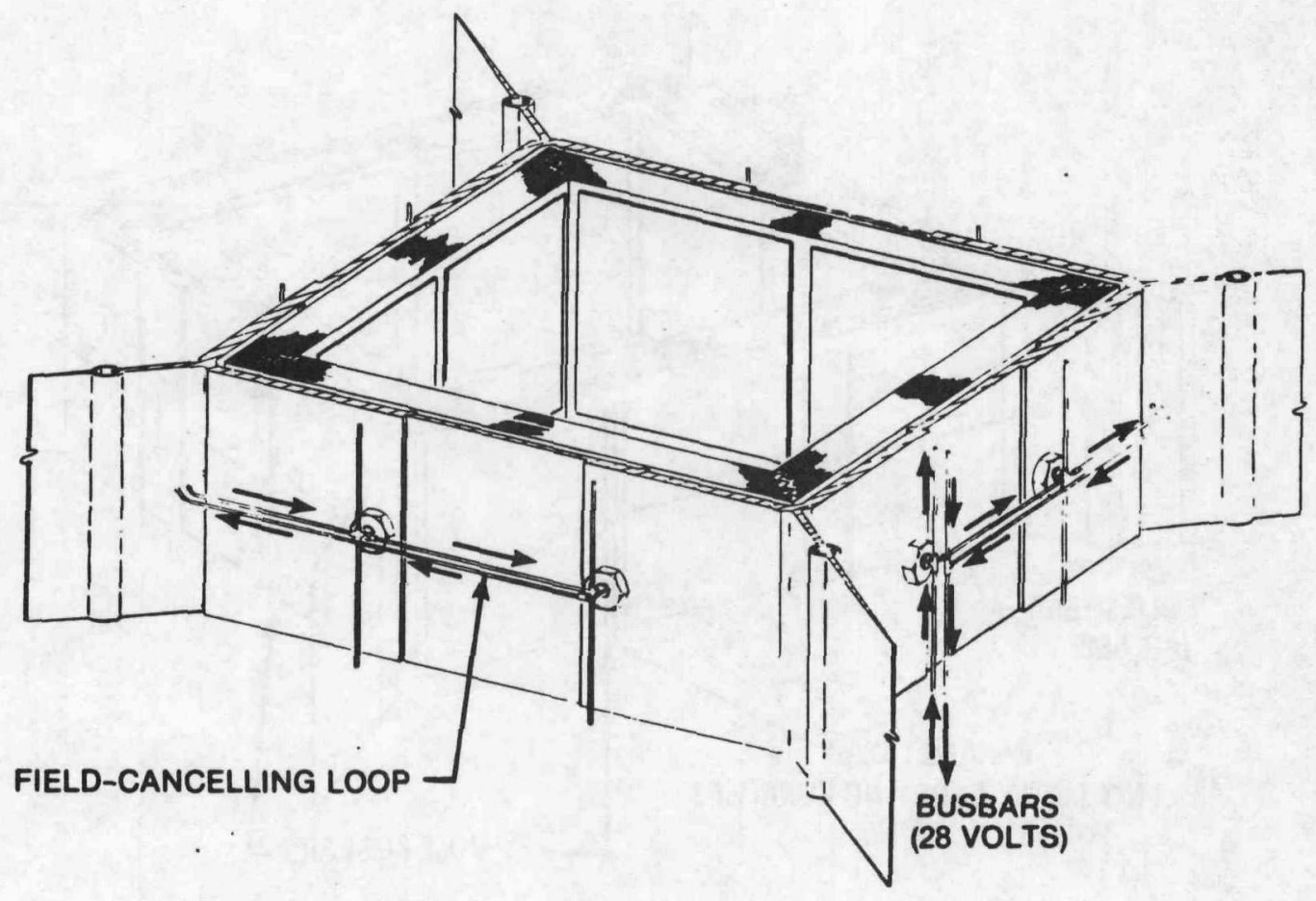

Figure 35. Standard Converter Slice with Field-Cancelling Circuit Module 
Since the electrical circuit in the MITG is located outside the generator housing, there is some concern about the effect of micrometeorite impacts on circuit integrity. This problem was studied using the analytical model for meteorite impacts for NASA's SolarPolar Mission. That model is displayed in Figure 36, which shows a plot of meteorite fluence versus particle mass.

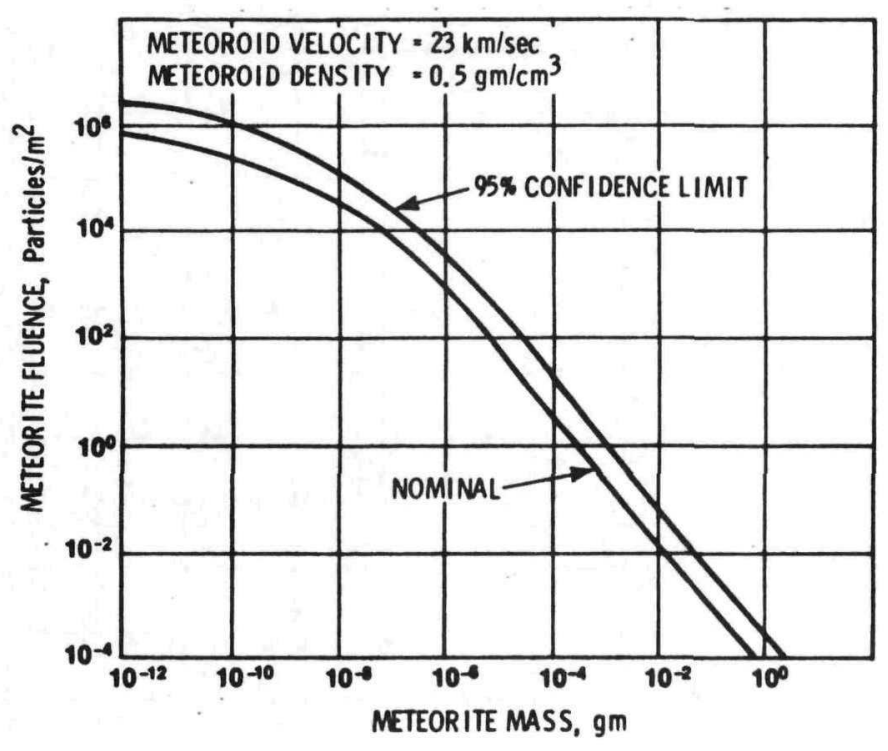

Figure 36. ISPM Meteorite Environment Model 
The study showed that the reliability of the circuit against micrometeorlte effects can be greatly enhanced by simple additions to the basic circuit shown earlier in Figure 34 . Figure 37 shows a schematic diagram of the complete circuit for a 12-slice generator, with the 96 thermoelectric modules arranged in a $8 \times 12$ seriesparallel array. The basic circuit elements are shown by solid lines, and the circuit additions for enhanced rellability against meteorites are shown by dashed lines. These additions consist of two items: parallelization of the busbars, with cross connectors between the two parallel busbar leads at each of the 12 slices; and the addition of parallel connectors at the second terminal of each of the thermoelectric modules.

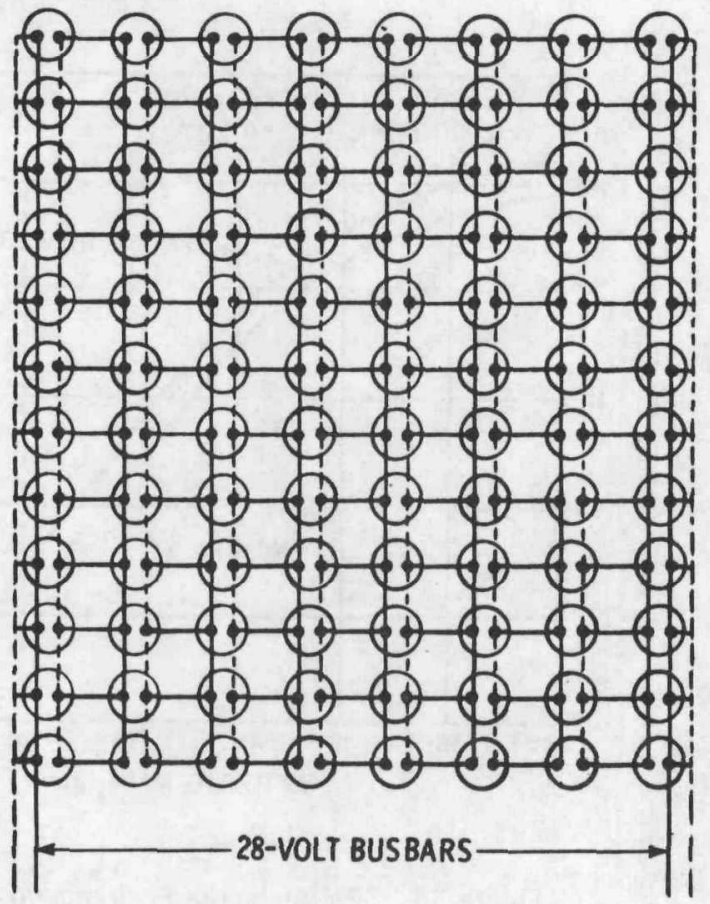

Figure 37. Redundant Circuit Additions ) for Enhanced Reliability Against Meteorites

The benefit of the redundant busbars is obvious, since-without them-micrometeorite impacts could cut the single busbar and cause a major fraction of thermoelectric modules to be lost from the circuit. As we will see, this is far less likely with redundant busbars.

The addition of the other redundant connectors (shown by dashed lines) is less important, but also beneficial. Without those redundant parallel cross-connectors, the loss of any series-connector would terminate the contribution of the thermoelectric module to the left of that connector. With the addition of the redundant parallel connectors, loss of a thermoelectric module would only occur if all three wires to one of its terminals were broken, an extremely unlikely event. 
The analytical predictions for meteorite impact effects on both the basic circuit and the redundant (improved) design are summarized in Table 1. For each option, the table lists the results for the busbars and for the remainder of the network. The table lists the wire gauge and diameter of the stranded connectors, as well as the resultant circuit weight and ohmic loss.

Table 1. Influence of Circuit Design on Meteorite Impact Effects

\begin{tabular}{|c|c|c|c|c|}
\hline \multirow[t]{2}{*}{$\begin{array}{l}\text { Based On Meteorite Model } \\
\text { For Solar-Polar Mission }\end{array}$} & \multicolumn{2}{|c|}{ BASIC DESIGN } & \multicolumn{2}{|c|}{ REDUNDANT DESIGHI } \\
\hline & BUSBARS & NETWORK & BUSBARS & NETWORK \\
\hline Parallel Wires & i & 12 & 2 & 12 \\
\hline Wire Gauge (stranded) & $\$ 9$ & 121 & $\$ 12$ & 121 \\
\hline Wire Diameter (equivalent) & 0.114 in & Q. 028 in & Q. 081 in & 0.028 in \\
\hline Wire Length (total) & $4 \mathrm{ft}$ & $39 \mathrm{ft}$ & $8 \mathrm{ft}$ & $55 \mathrm{ft}$ \\
\hline Wire Weight & Q. $16 \mathrm{lb}$ & Q. 19 Ib & $0.16 \mathrm{lb}$ & 0.2716 \\
\hline Ohmic Loss & $\begin{array}{l}0.087 \% \\
0.24 \%\end{array}$ & $\begin{array}{l}0.0927 \\
0.26 w\end{array}$ & $\begin{array}{l}0.087 \% \\
0.24 w\end{array}$ & $\begin{array}{l}0.0927 \\
0.26 w\end{array}$ \\
\hline Total-Failure Probability* & 0.00072 & Negligible & 0.00000013 & Negligible \\
\hline Survival Probability* & 0.9993 & $\sim 1$ & Q.9999999 & $\sim 1$ \\
\hline Probability of Losing One or More T/E Modules & - & a. 106 & - & 0.000039 \\
\hline Non-Degradation* Probability & - & 0.89 . & - & 0.99996 \\
\hline
\end{tabular}

-due to micrometeoroid impacts on circuit

As can be seen, the circuit weight is very low for all cases, because of the low current handled. Similarly, the ohmic loss is less than $0.2 \%$ of the generated power, for either option. The analytical results show that the probability of not encountering a break in any of the busbars is quite high (three 9's) even for the basic circuit design, and is extremely high (seven 9's) for the redundant design. The probability of total failure due to damage of the rest of the network is negligible for both designs. Finally, the probability of losing the power output of one or more of the generator's 96 thermoelectric modules (due to micrometeorite impact) is $11 \%$ for the basic circuit design, and only $0.004 \%$ for the redundant design. 
Clearly, the redundant design is worth using, since it adds minimal weight and no ohmic losses to the system, and yields a very high reliability. A typical converter slice with the redundant circuit is deplcted in Figure 38. It shows the duplicate busbars and the added parallel connectors.

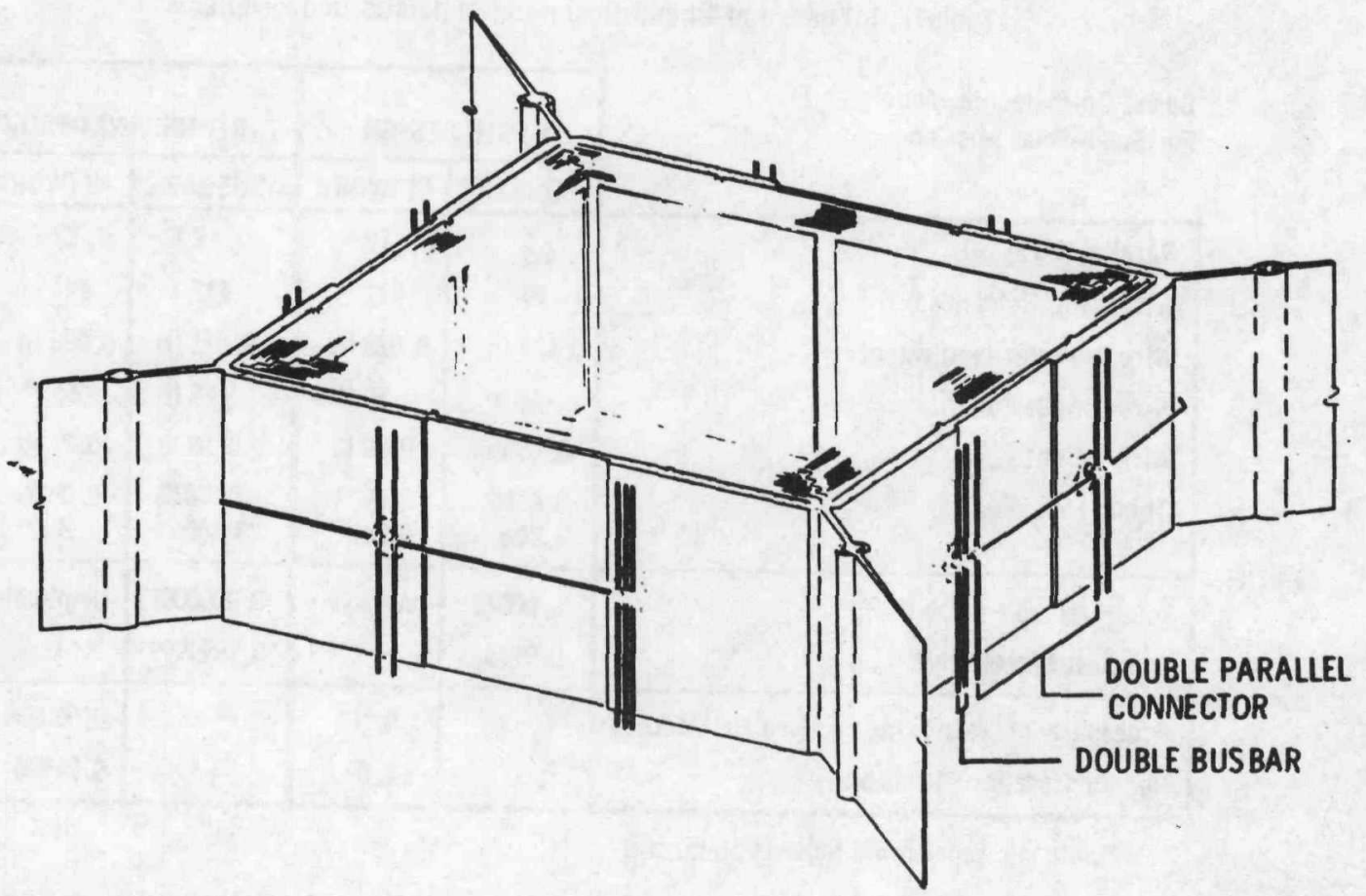

Figure 38. Standard Converter Slice with Redundant Circuit

One other circuit option should be mentioned. Historically, RTGs have been designed for an output of 28 to 30 volt, because much of the available payload equipment falls into that range. However, there are many applications, particularly at higher power levels, in which only the housekeeping equipment operates at that voltage, while the major power consumers of the payload operate at a much higher voltage. With the multicouple design, the circuit could readily be modified to provide much higher output voltages; e.g., up to $180 \mathrm{~V}$ at $300 \mathrm{~W}$. Alternatively, the circuit could be arranged to deliver part of the power at 28 volts for housekeeping, and part at a higher voltage for the other payload. 


\section{HEAT SOURCE SUPPORT}

After the electrical circuit has been installed on the outside of the generator housing, the converter is ready for fueling, using the heat source modules pictured earlier in Figure 1. Figure 39 shows a typical slice of the MITG generator, producing approximately 24 watt at 28 volt. An MITG design for a given power output is obtained by combining an appropriate number of such standard slices, and adding the necessary end sections to complete the generator.

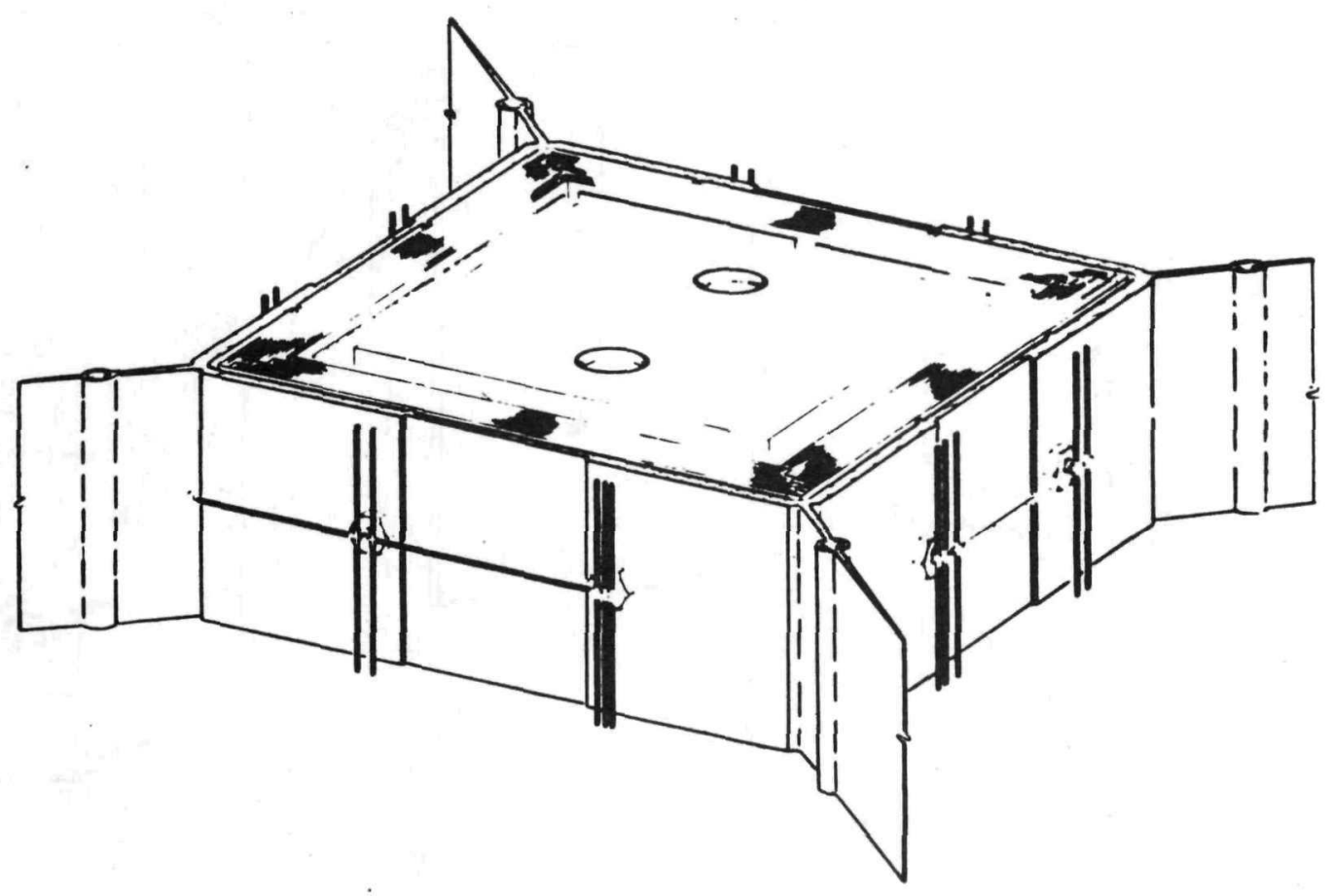

Figure 39. Standard MITG Slice ( $\sim 24$ Watts at 28 Volt) 
Figure 40 shows a view of the generator housing, including its end flanges, the mounting bushings at the base of the fins, and the auxiliary coolant loop. This coolant loop is not needed in space, but serves to maintain the generator housing within prescribed temperature limits while it is within the shuttle bay.

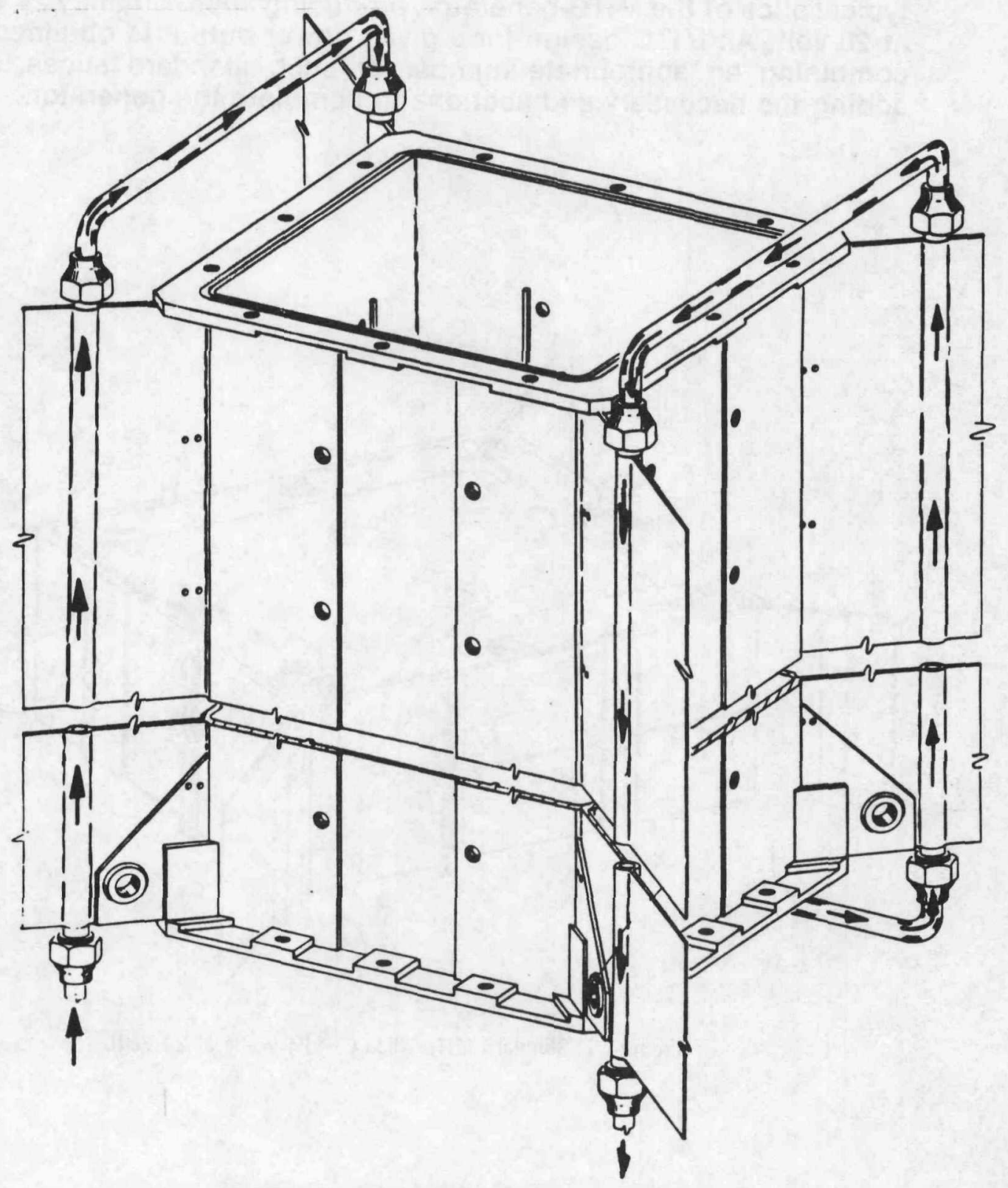

Figure 40. MITG Housing, with Auxiliary Coolant Ducts 
Figure 41 depicts a full-length generator housing, together with a typical heat source consisting of 12 GPHS modules. Structural support of this heat source stack is a critical problem, since it is only supported at its ends and there are no intermediate lateral supports. Very high axial preloads, on the order of $3000 \mathrm{lbs}$, are required to keep the modules from coming apart under the influence of the transverse g-loads experienced during launch (typically $\mathbf{4 0} \mathrm{grms}$ ). This problem was discussed in great detail in earlier Fairchild papers [13, 14], and only the MITG design solution will be presented here.
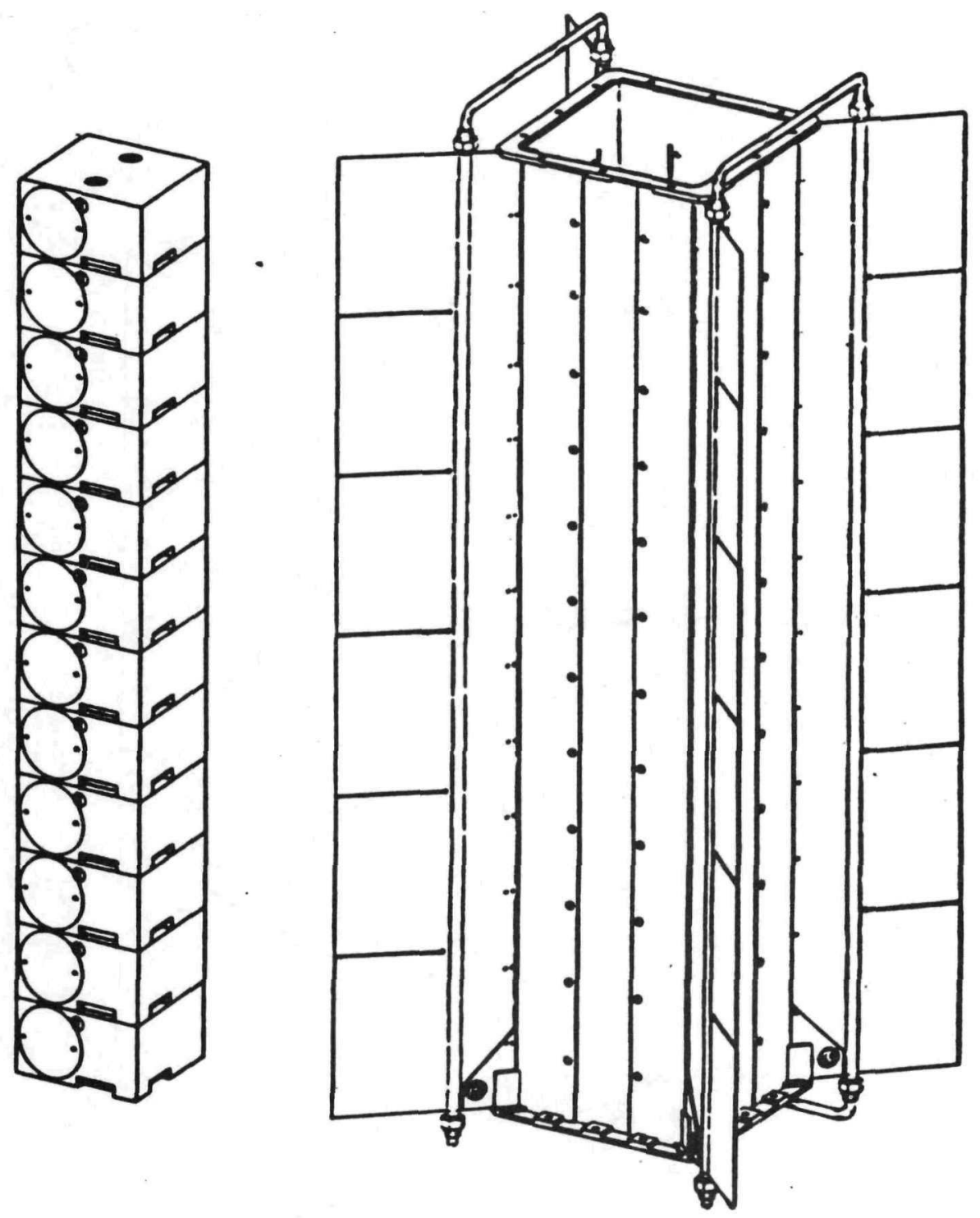

Figure 41. Heat Source Stack and Generator Housing 
The basic heat source support hardware is depicted in Figure 42. The two heat source modules shown represent, of course, a much longer stack. At each corner of the heat source stack is a refractorymetal load spreader, which is contacted by a pyrolytic graphite button. Pyrolytic graphite is an excellent support material, because of its very low thermal conductivity in the $\mathrm{C}$-direction (normal to the deposition plane) and because of its high strength at high temperatures. By orienting these buttons, in a space-diagonal direction as shown, the required number of supports is reduced to a minimum.

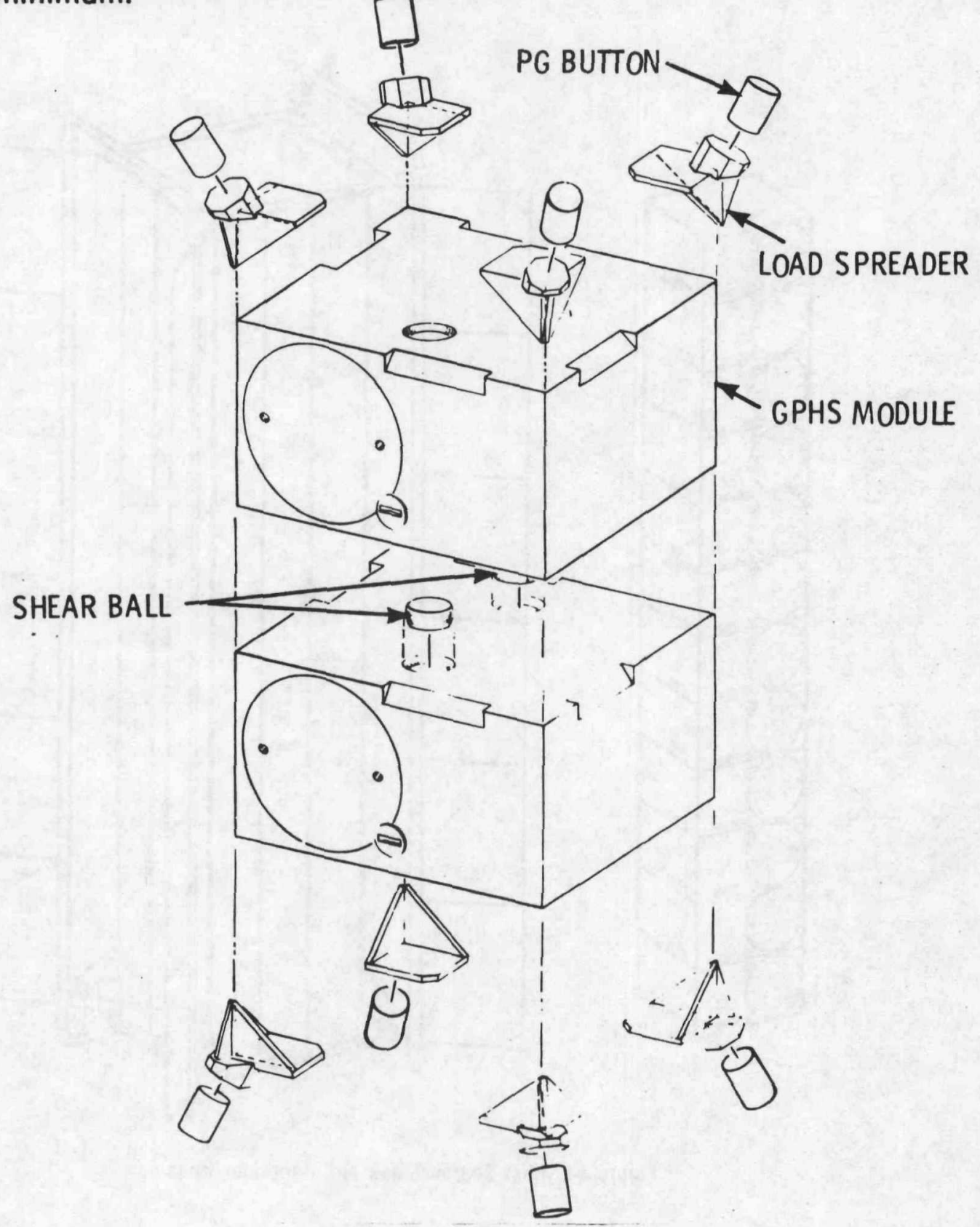

Figure 42. Heat Source Support Hardware 
The preload forces are applied by Belleville springs, as Illustrated in Figure 43. Note that only the upper corners of the heat source are spring-loaded. The bottom ones are rigidly connected to the generator housing.

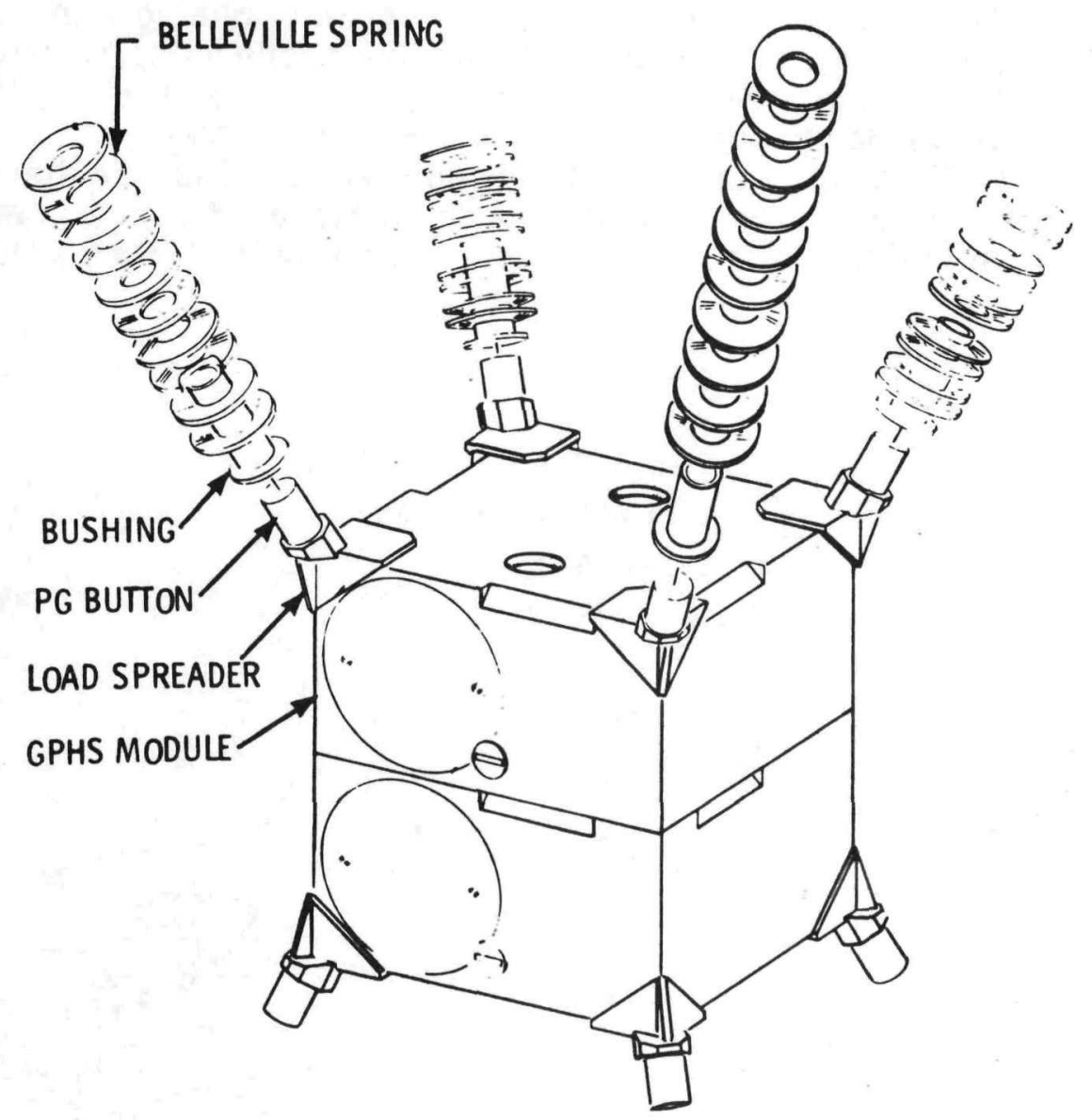

Figure 43. Heat Source Preload Springs

Belleville springs are very well-suited to this application because of their compactness and ability to deliver relatively high forces. Not too much travel is required in the present application, just enough to compensate for differential thermal growth of the heat source stack and generator housing. As shown, a total of 10 Belleville springs is required at each of the upper corners, arranged in five pairs of two. Each pair contains two springs in parallel, to double the available force. The five pairs are stacked in series, to quintuple the available travel. 
Figure 44 deplcts the generator end caps and the relation of the preload hardware to the top cap. As can be seen, the load-bearing points on the end cap are located near the housing flange. This is desirable because it results in primarily-tensile loads, with relatively low bending moments on the end caps. As a result, a very light end cap design is possible: basically a 0.090 "--thick plate with two 0.660 " by 0.090 " stiffening ribs. The structural adequacy of the end cap design shown, for a 3500-lb axial preload and a 1-atmosphere pressure differential, has been confirmed by NASTRAN analysis.

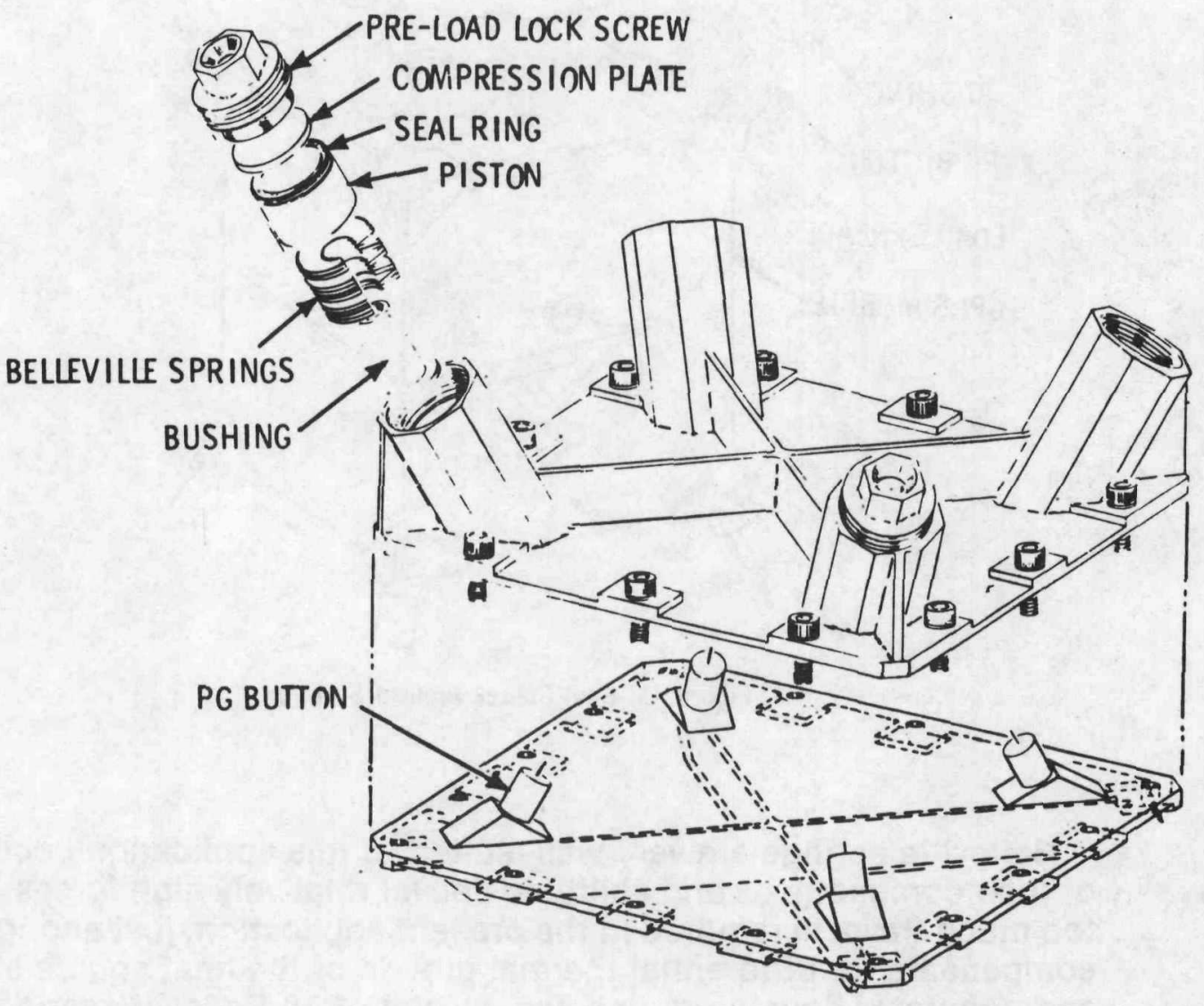

Figure 44. End Covers and Preload Hardware 
The detailed preload hardware is depicted in exploded view at one corner of the top cap in Figure 44 and in assembled form in the closeup section shown in Figure 45 . The pyrolytic graphite button at the top of the heat source stack contacts a bushing, which is loaded by the five pairs of Belleville springs, as shown. These springs bear against a piston, which is locked in position by the preload lock screw.

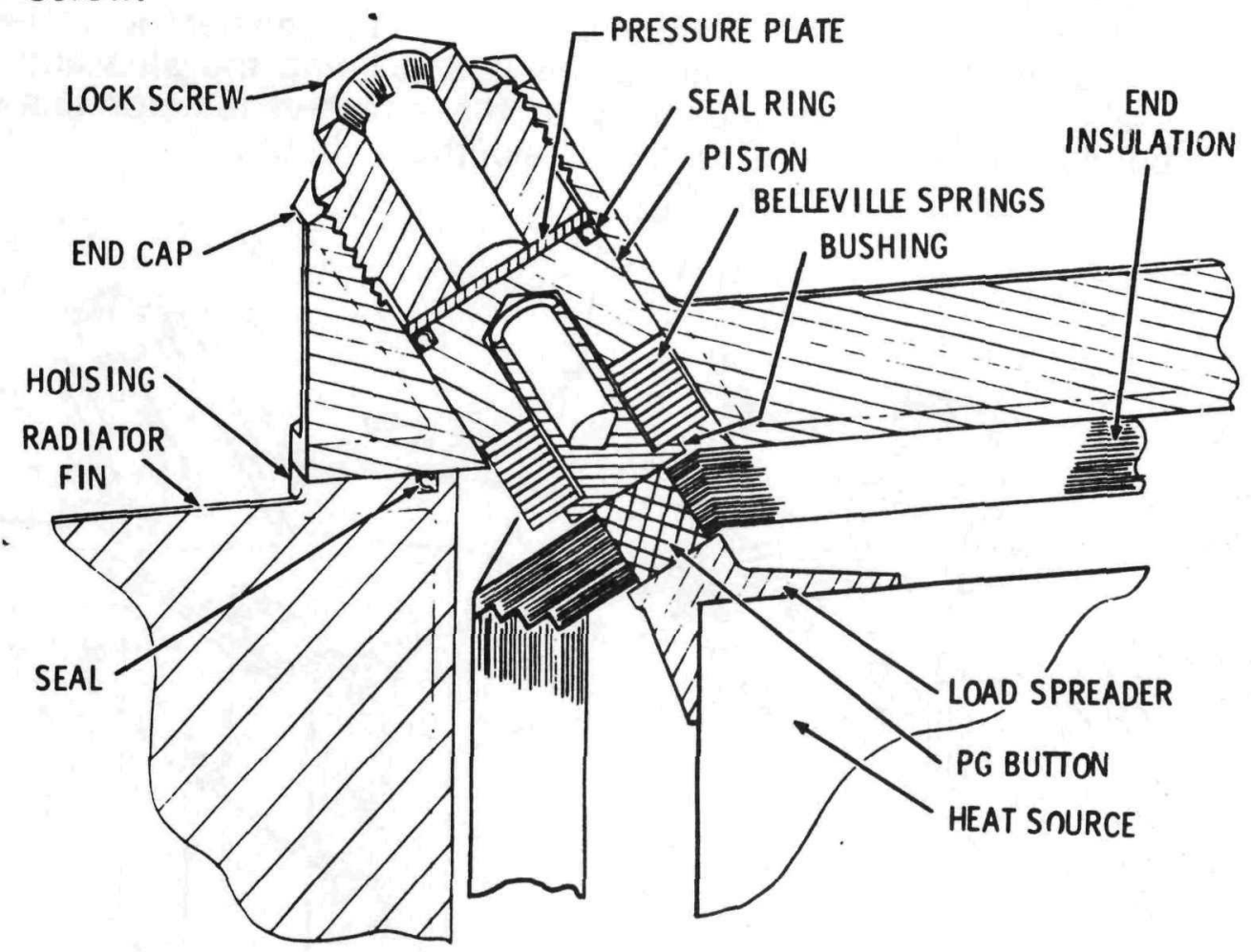

Figure 45. Close-Up of RTG Corner, Showing Assembled Preload Hardware

The arrangement is designed to permit final adjustment of the preload forces after the generator is assembled and its various parts have reached their equilibrium temperature. The desired preload at each corner is applied via a pneumatic actuator or a load cell. The load is applied to the compression plate by passing a rod through the central hole in the preload lock screw. After the load is applied and the springs have been compressed, the lock screw is tightened and the load device removed.

The ability to adjust the preload at each corner in situ (i.e., in the fully assembled and thermally equilibrated generator) is very important. It makes it possible to compensate for the dimensional deviation of the actual hardware from lits nominal dimensions. This should allow relaxation of the tolerance specifications for various components. 


\section{GENERATOR ASSEMBLY}

Figure 46 presents a cutaway view of the upper end of the assembled generator. It illustrates the geometric relationship of the heat source stack and its end supports, the thermoelectric converter modules, the multifoil thermal insulation lining the side and end walls, the generator housing with the attached radiator fins and bolted end caps, and the external electrical circuit.

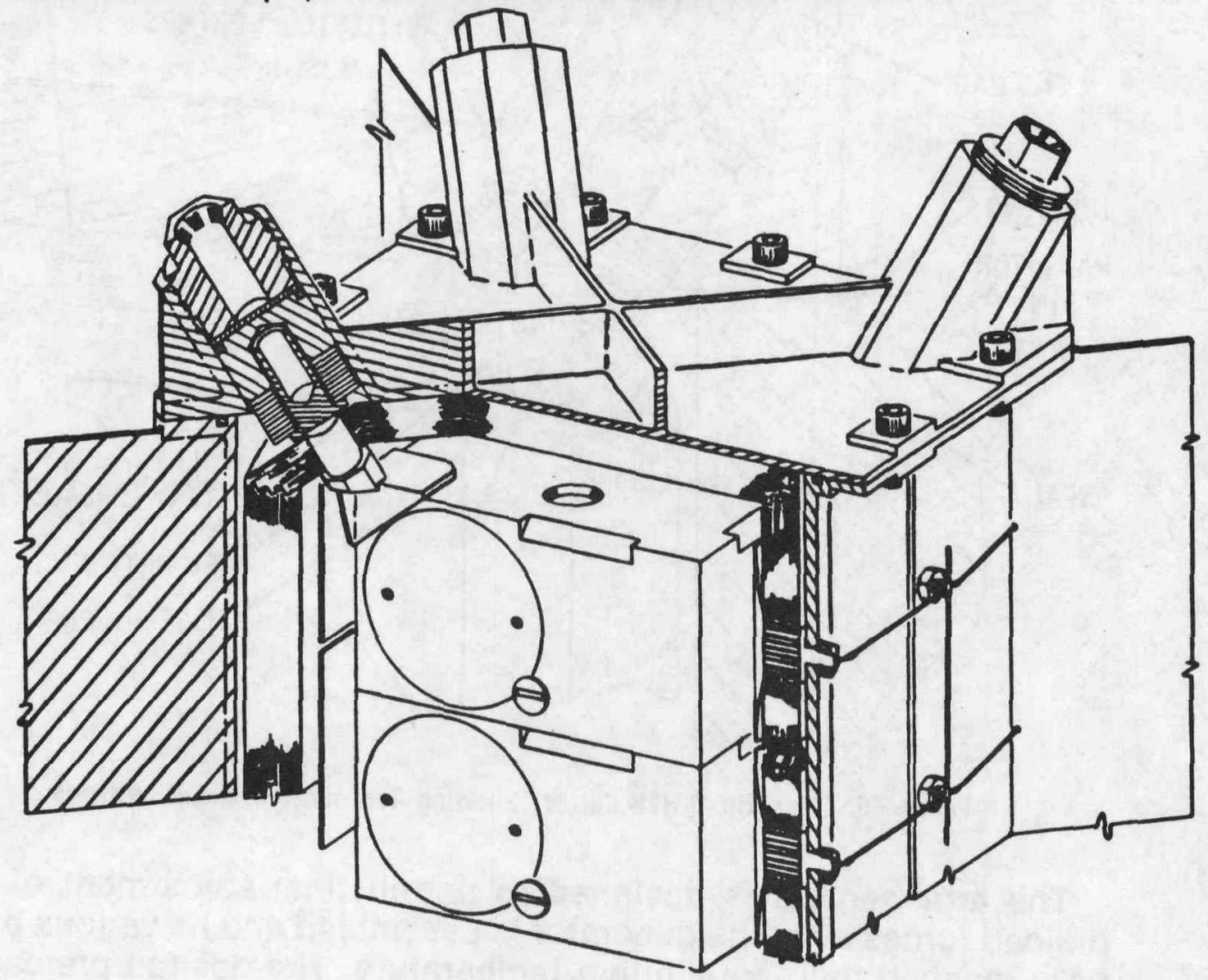

Figure 46. Cut-Away View of MITG End Section

The figure illustrates how compact and densely packed the MITG design is. The dimensions of the housing have been reduced to a near-minimum. This accounts for the low weight and high specific power of the generator. 
A view of the fully-assembled generator is shown in Figure 47. The figure depicts the auxiliary coolant loop, and shows the mounting bushing at the base of each fin. These bushings are used for structural attachment of the cantilevered generator to the spacecraft. The electrical circuit illustrated in the figure combines the magnetic field-cancelling loops with the redundant-circuit elements added for enhanced reliability against micrometeorite impact. It shows the double busbars and the 28-volt generator terminals.

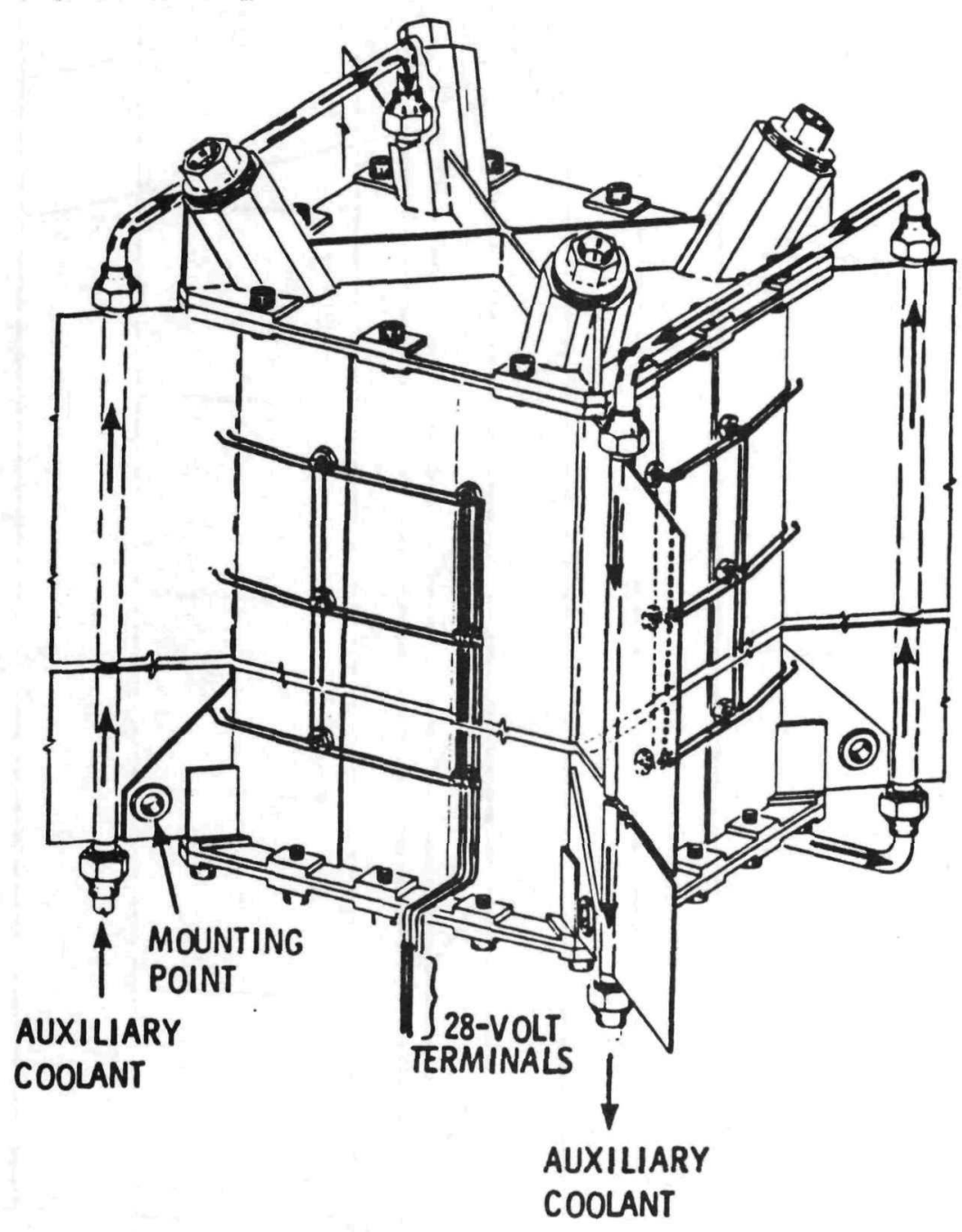

Figure 47. External View of Assembled MITG 
Finally, Figure $\mathbf{4 8}$ gives the overall dimensions of a typical 12-slice MITG (shown without electrical circuit or auxiliary coolant loop). Note that the radiator fins are slotted at 4" intervals, to reduce thermal stresses caused by temperature gradients.

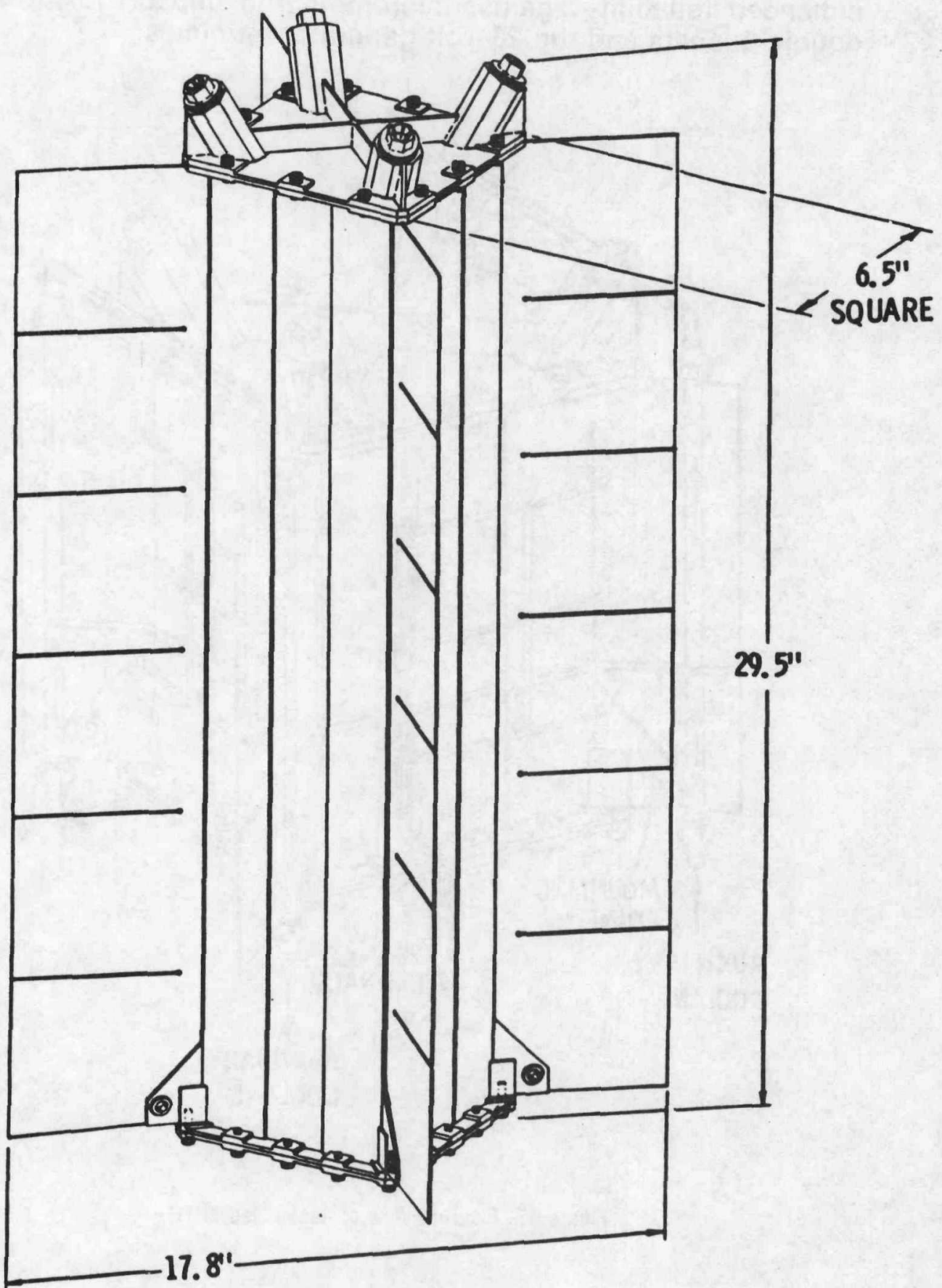

Figure 48. Typical MITG Dimensions (12 Slices, 282 Watts) 


\section{WEIGHT COMPARISON}

A weight summary of all the components of the generator, including some which have not been discussed in this paper, is shown in Table 2. The table presents a detailed weight breakdown for the GPHS/RTG as well as one for a 12-slice MITG with a similar power output. Combining these breakdowns minimizes the chances of overlooking some minor or peripheral components, and facilitates comparison of the two designs. The weights of the GPHS/RTG are shown at the left of the table, the weights of the corresponding MITG components are shown at right, and the middle column shows a breakdown of the MITG weight savings.

Table 2. Weights (Lb)

\begin{tabular}{|c|c|c|c|c|c|c|}
\hline \multicolumn{3}{|c|}{ GPHS RTG (SiGe, 290 w) * } & & \multicolumn{3}{|c|}{ MITG (SiGe/GaP, 282 w) } \\
\hline Item & No. & Wt & & Wh & Na. & Item \\
\hline $\begin{array}{l}\text { Housing: } \\
\text { Outer Shell } \\
\text { Fins } \\
\text { Emissive Coating } \\
\text { Auxiliary Cooling Tube Manifold } \\
\text { Nuts }\end{array}$ & $\begin{array}{l}1 \\
8 \\
x \\
1 \\
8\end{array}$ & $\begin{array}{r}14.25 \\
4.32 \\
0.33 \\
0.57 \\
0.11\end{array}$ & $\begin{array}{l}10.66 \\
8.25 \\
2.02 \\
0.08 \\
0.27 \\
0.04\end{array}$ & $\begin{array}{l}6.00 \\
2.30 \\
0.25 \\
0.30 \\
0.07\end{array}$ & $\begin{array}{l}1 \\
4 \\
x \\
1 \\
4\end{array}$ & \\
\hline Converter: & & & 25,82 & & & \\
\hline $\begin{array}{l}\text { T/E Unicouples } \\
\text { T/E Sealing Screws } \\
\text { T/E C-Seals } \\
\text { Rivets and Washers } \\
\text { T/E Spacers }\left(\mathrm{Al}_{2} \mathrm{O}_{3}\right) \\
\text { Nut Plates }\end{array}$ & $\begin{array}{l}576 \\
576 \\
576 \\
608 \\
576 \\
288\end{array}$ & $\begin{array}{r}11.98 \\
1.54 \\
0.13 \\
0.59 \\
0.64 \\
0.80\end{array}$ & $\begin{array}{l}8.18 \\
1.54 \\
0.10 \\
0.59 \\
0.64 \\
0.45\end{array}$ & $\begin{array}{c}3.80 \\
0.03 \\
- \\
0.35\end{array}$ & $\begin{array}{l}\% 0 \\
\dot{-} \\
\dot{-} \\
\dot{-}\end{array}$ & $\begin{array}{l}\text { TE Modules } \\
\text { Nuts }\end{array}$ \\
\hline $\begin{array}{l}\text { Foil Insulation - T/P } \\
\text { Foil Insulation - Ends } \\
\text { Insulation Support Frame } \\
\text { Power Connector } \\
\text { Gas Management Assembly } \\
\text { Electrical Straps }\end{array}$ & $\begin{array}{l}1 \\
2 \\
1 \\
1 \\
1 \\
x\end{array}$ & $\begin{array}{l}13.00 \\
1.33 \\
1.65 \\
0.35 \\
0.29 \\
1.08\end{array}$ & $\begin{array}{l}10.90 \\
1.13 \\
1.65 \\
0.68\end{array}$ & $\begin{array}{l}2.10 \\
0.20 \\
- \\
0.35 \\
0.29 \\
0.40\end{array}$ & $\begin{array}{l}1 \\
2 \\
- \\
1 \\
1 \\
x\end{array}$ & s. \\
\hline $\begin{array}{l}\text { PRD } \\
\text { C-Seal - Domes } \\
\text { Other Insulation } \\
\text { Pressure Dome } \\
\text { Screws - Pressure Dome } \\
\text { RTG Assembly }\end{array}$ & $\begin{array}{r}1 \\
2 \\
x \\
2 \\
44 \\
1\end{array}$ & $\begin{array}{l}0.90 \\
0.08 \\
0.20 \\
1.25 \\
0.23 \\
1.00\end{array}$ & $\begin{array}{r}0,02 \\
0.10 \\
-0,22 \\
0.06\end{array}$ & $\begin{array}{l}0.90 \\
0.06 \\
0.10 \\
1.47 \\
0.17 \\
1.00\end{array}$ & $\begin{array}{r}1 \\
2 \\
x \\
2 \\
32 \\
x\end{array}$ & $\begin{array}{l}\text { End Caps } \\
\text { Pads \& Bushings }\end{array}$ \\
\hline $\begin{array}{l}\text { Heat Source Support System: } \\
\text { Inboard Support } \\
\text { Mid-Span Support } \\
\text { Outboard Support }\end{array}$ & $\begin{array}{l}x \\
x\end{array}$ & $\begin{array}{l}4.77 \\
3.10 \\
1.85\end{array}$ & 841 & $\begin{array}{l}0.35 \\
0.04 \\
0.09 \\
0.14 \\
0.32 \\
0.06 \\
0.31\end{array}$ & $\begin{array}{r}8 \\
8 \\
8 \\
16 \\
8 \\
40 \\
8\end{array}$ & $\begin{array}{l}\text { Load Spreaders } \\
\text { PG Buttons } \\
\text { Bushings } \\
\text { Belleville Springs } \\
\text { Pistons } \\
\text { Compression Plates } \\
\text { Preload Screws }\end{array}$ \\
\hline Heat Source Modules, 4464 w(t) & 18 & 57.44 & 19,02 & 38.42 & 12 & $3000 \mathrm{w}(t)$ \\
\hline TOTAL & & 123.78 & 63.91 & 59.87 & & \\
\hline
\end{tabular}

-These weights were presented by GE at the Final Design Review of the GPHS/RTG in October 1980. They may change somewhat in the course of further development. 
As can be seen, there are substantial weight reductions in all four areas: the housing assembly, the converter, the heat source support system, and the heat source modules. The weight saving of the housing assembly is due to the greater compactness of the MITG design and to the better view of space from four fins instead of eight fins. In the converter, there are major weight savings due to the much smaller number of thermoelectric modules, and to the shorter leg length and simpler mounting arrangement of the thermoelectric multicouples.

There are very large weight savings in the foil insulation package, because of the elimination of quartz cloth spacers, and because the areas to be insulated are much smaller due to the compactness of the MITG. Additional weight savings result from the elimination of the insulation support frame, which is not required in the MITG design.

The MITG end caps are slightly heavier than the GPHS pressure domes, because the latter only serve to seal the housing but not to support the heat source. The GPHSIRTG employs separate titanium spiders for heat source support, and their weight is accounted for as part of the heat source support system. No such spiders are required in the MITG, since the end caps fulfill that function. This and the greater compactness of the generator account for the much lower weight of the MITG heat source support system.

The heat source weight saving is simply due to the higher system efficiency of the MITG, which allows roughly the same electrical output to be achieved with 12 instead of 18 heat source modules. As will be seen, the high system efficiency is due primarily to the high material efficiency of SiGe/GaP, and secondarily to the high thermal efficiency of the very compact and snugly insulated design.

Overall, the MITG design shown weighs only about half as much as the GPHSIRTG. 
Table 2. Weights (Lb)

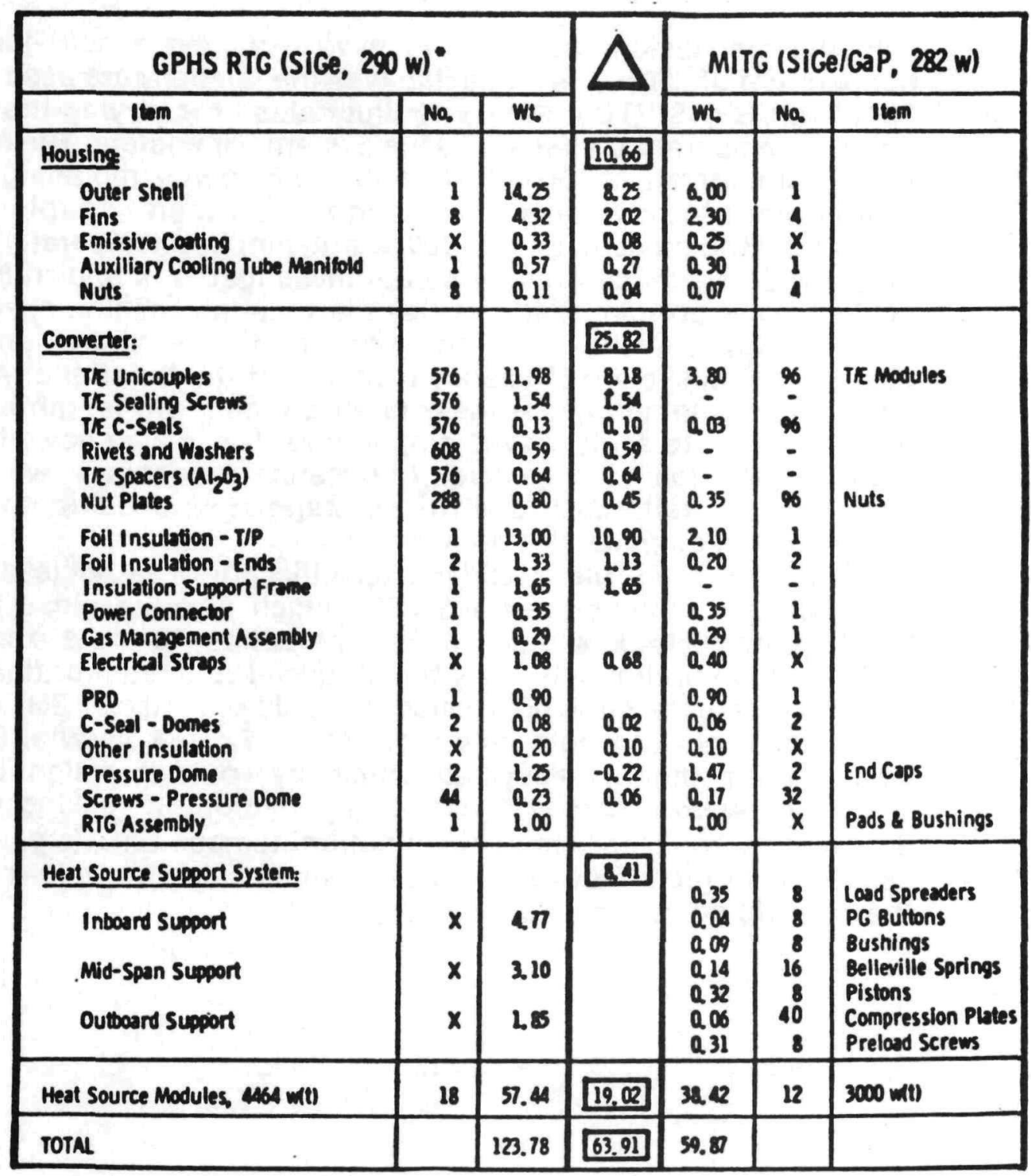

"These weights were presented by GE at the Final Design Review of the GPHS/RTG in October 1980. They may change somewhat in the course of further development. 


\section{EFFECT OF COLD-JUNCTION TEMPERATURE}

In the discussion thus far, we have assumed a cold-junction temperature of $300^{\circ} \mathrm{C}$, because that was the temperature used in the MHW and GPHS RTGs. Figure 49 illustrates how varying the coldjunction temperature affects various system parameters. The results shown are for an MITG with twelve heat source modules, eight thermoelectric modules per slice, eighteen couples per thermoelectric module, and a $1000^{\circ} \mathrm{C}$ hot-junction temperature. The range of cold-junction temperatures investigated is shown by tick marks at the bottom of the curve; the resultant beginning-of-life power output is shown at the bottom of the figure; and the corresponding system efficiency is shown at the top. For any given cold-junction temperature, there is an optimum fin length and fin root thickness to achleve that temperature. These are shown by tick marks at the top of the curve. The resultant generator weight is shown by the left scale, and the corresponding specific power is shown by the diagonal dotted lines.

As can be seen, the maximum specific power is achieved at a cold-junction temperature of $310^{\circ} \mathrm{C}$, which is quite close to the $300^{\circ} \mathrm{C}$ previously assumed. It is interesting to note that the cold-junction optimum is fairly broad. Even if the radiator fins were omitted completely, the cold junction would only rise to $350^{\circ} \mathrm{C}$, and the specific power would only drop from 4.72 to $4.58 \mathrm{w} / \mathrm{lb}$. But, of course, power output and system efficiency would drop significantly if the fins were omitted. In the other direction, increasing the fin dimensions to lower the cold-junction temperature (e.g., below $280^{\circ} \mathrm{C}$ ) is not too effective, because the increasing fin weight makes the specific power drop off sharply. 


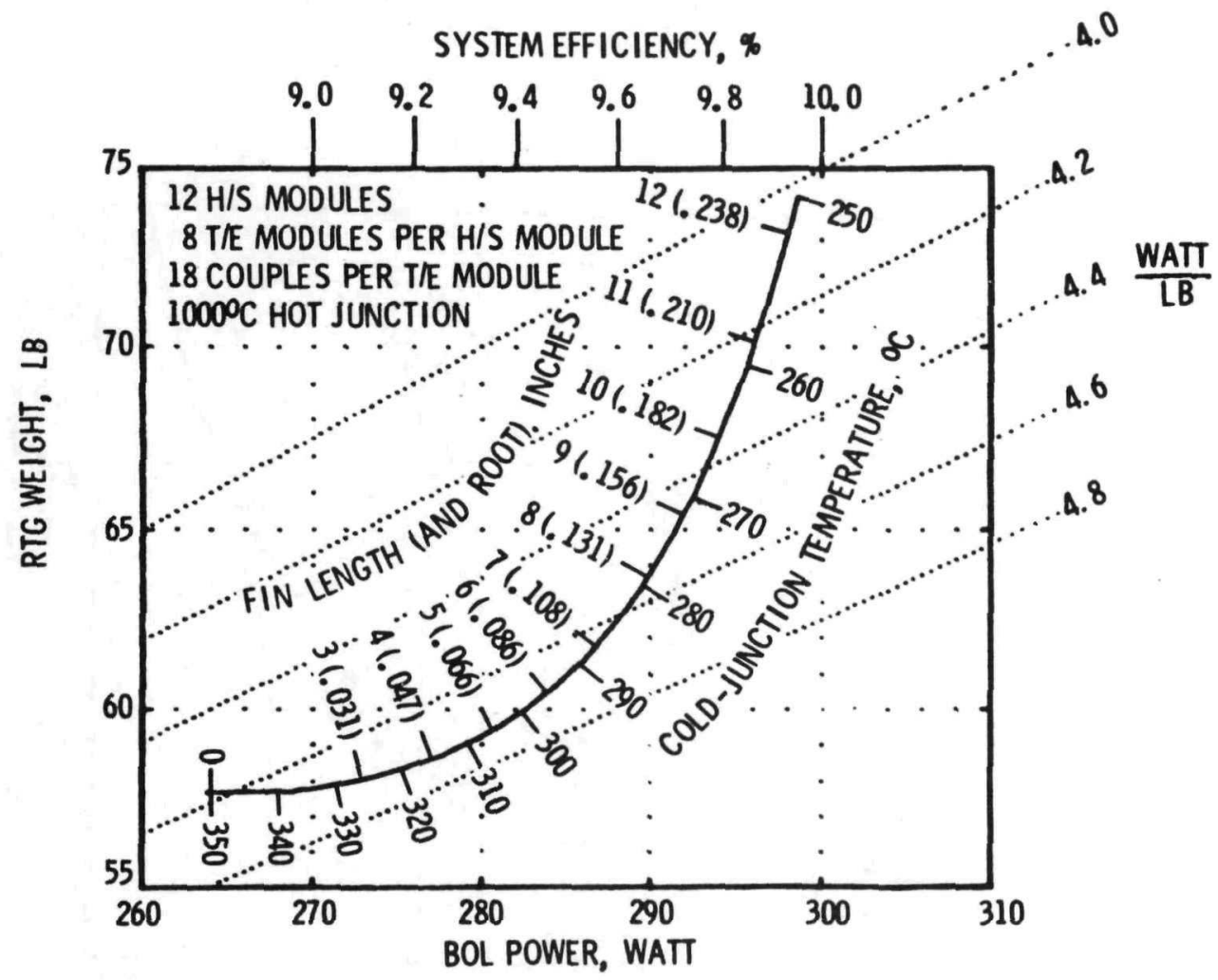

Figure 49. Effect of Cold-Junction Temperature on Optimum Fin Dimensions. RTG Weight \& Power, System Efficiency, and Specify Power 
The weight-vs-power curve shown in Figure 49 is for a 12-slice MITG. This curve is compared with similar curves for 11- and 13-slice MITGs in Figure 50. That figure also has the diagonal lines of constant specific power. As can be seen, for any given power level there is an optimum number of generator slices which minimizes the MITG weight.

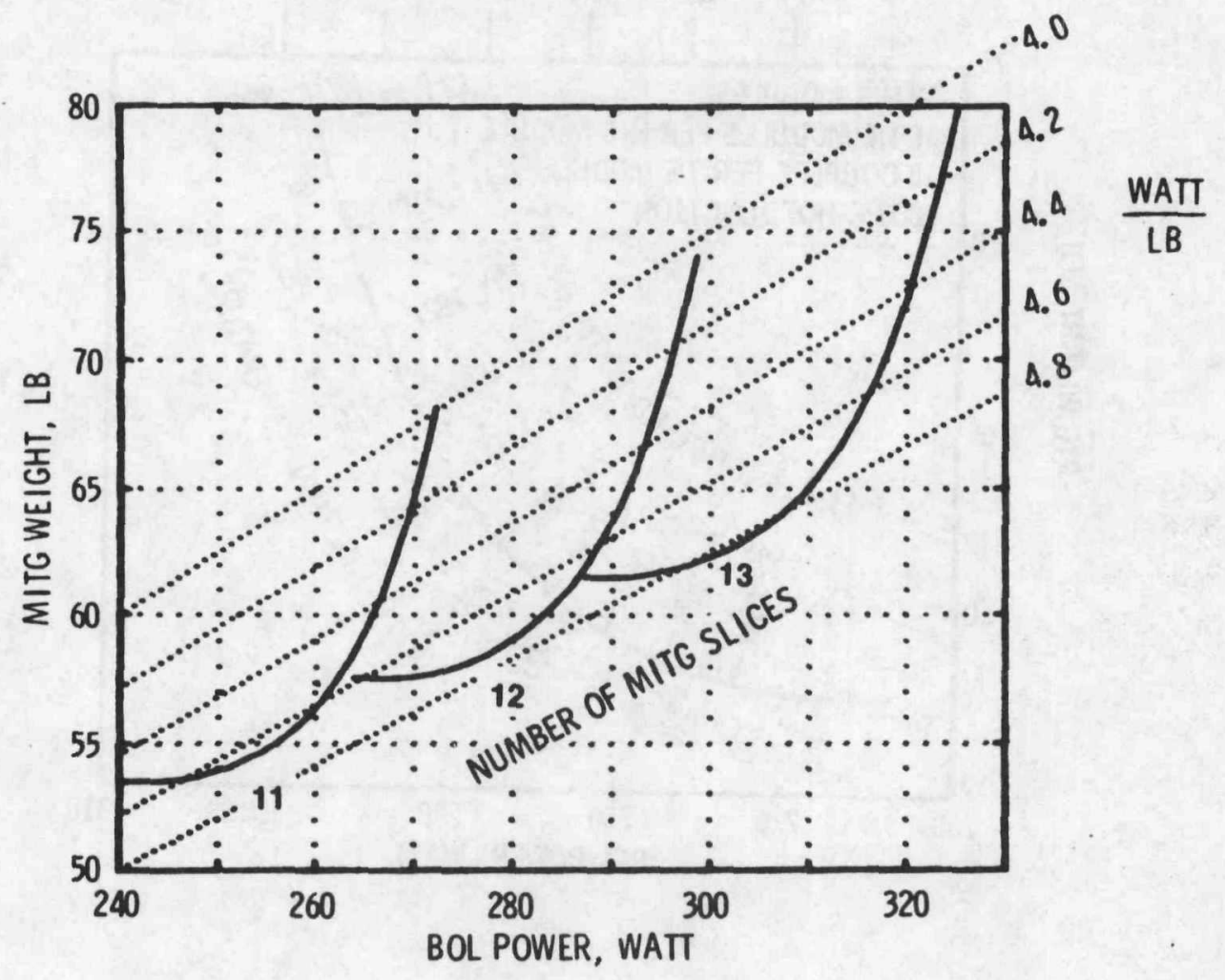

Figure 50. Weight-vs-Power Curves for Various Numbers of MITG Slices

The three curves shown are part of a much larger family of curves, and the scalloped envelop of those curves represents the maximum specific power for any given power level for the MITG design. Not surprisingly, the specific power tends to increase with power level, since the weight of the generator end sections and the associated heat losses become less significant for larger generators. The important point to note is that any desired power level can be achieved. Even though the heat source comes in discrete modules of 250 watts, the generator's power output is infinitely variable by adjusting the radiator fin dimensions. 


\section{ILLUSTRATIVE DESIGN SUMMARY}

Table 3 presents a design summary for the 12-slice MITG with a $300^{\circ} \mathrm{C}$ cold-junction temperature. It lists the number of components, principal dimensions, critical temperatures, and temperature drops. Note the large temperature drop between the fuel capsule and the heat source surface, and that between the hot-shoe corner and the hot-shoe root. The cold-end temperature drops are relatively modest in this design, but the temperature drop in the optimized radiator fins is significant. As can be seen, the total temperature drop from the fuel capsule is about $1000^{\circ} \mathrm{C}$ to the generator housing, and $1100^{\circ} \mathrm{C}$ to the radiator fin tip; only $700^{\circ} \mathrm{C}$ of this is a productive temperature drop, in the thermoelectric legs.

Table 3. Illustrative Design Summary (BOL)

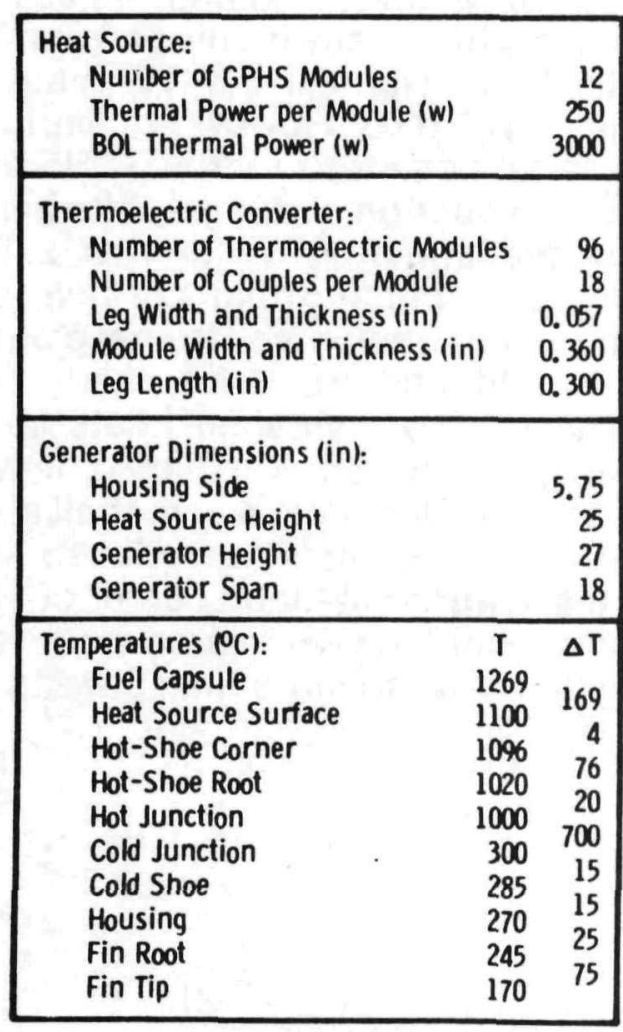

\begin{tabular}{|c|c|}
\hline $\begin{array}{l}\text { Heat Balance (w): } \\
\text { Thermal Power } \\
\text { End Losses } \\
\text { Side Losses } \\
\text { Heat to TE Modules } \\
\text { Heat from TE Modules } \\
\text { Total Heat Rejection }\end{array}$ & $\begin{array}{r}3000 \\
75 \\
100 \\
2825 \\
2543 \\
2718\end{array}$ \\
\hline $\begin{array}{l}\text { Electrical: } \\
\text { Couples in jeries } \\
\text { Couples in Parallel } \\
\text { Module Voltage } \\
\text { Module Current (amp) } \\
\text { Generator Voltage } \\
\text { Generator Current (amp) } \\
\text { Power Output (w) }\end{array}$ & $\begin{array}{r}144 \\
12 \\
3.50 \\
0.84 \\
28 \\
10.1 \\
282\end{array}$ \\
\hline $\begin{array}{l}\text { Efficiency (\%): } \\
\text { Thermal (excl. module losses) } \\
\text { Thermoelectric Material } \\
\text { Thermoelectric Module } \\
\text { System }\end{array}$ & $\begin{array}{r}94 \\
10.5 \\
10.0 \\
9.4\end{array}$ \\
\hline $\begin{array}{l}\text { Weight (lbs): } \\
\text { Heat Source } \\
\text { Converter } \\
\text { Total }\end{array}$ & $\begin{array}{l}38.4 \\
21.5 \\
59.9\end{array}$ \\
\hline Specific Power (w/lb): & 4.71 \\
\hline
\end{tabular}

Aeroshell fillets removed, and walls reduced to twice the predicted recession 
The table also presents a heat balance, showing the heat losses through the end and side insulation (including the pyrographite heat source buttons) to be quite small. This accounts for the high thermal efficiency of the generator $(94 \%)$.

The next block of data describes the electrical circuit, showing a module current of only 0.84 amps and a beginning-of-life power output of 282 watt. The SiGe/GaP multicouples have a material efficiency of $10.5 \%$ and a module efficiency of $10.0 \%$. The difference between these represents both the electrical losses due to contact resistance at the hot- and cold-junctions, and the thermal losses due to heat conduction through the $\mathrm{SiO}_{2}$ insulation. The thermal efficiency and module efficiency yield a system efficiency of $9.4 \%$ for the design. Finally, as shown earlier in Table 2, the 12-slice MITG weights $59.9 \mathrm{lb}$, and its specific power is $4.71 \mathrm{w} / \mathrm{lb}$.

All of the numbers presented above have assumed the use of the present GPHS heat source module without any modifications. This is to permit consistent comparisons between the MITG results and corresponding numbers for the GPHS/RTG. However, for future use it is possible to identify weight reduction steps for the GPHS module [5]. Principally, this would involve reduction of the graphite aeroshell wall from 0.185 " to $0.120 "$, and elimination of internal aeroshell fillets. There are good reasons for expecting that the heat source module would still meet its safety requirements with these changes. The reduced wall thickness would still be twice the predicted ablation during reentry, and the internal aeroshell fillets serve no obvious function (see Figure 1). Their removal would leave the aeroshell as a hollow rectangular box. Once these aeroshell changes passed safety qualification, they would result in the reduced weights shown in the small column at the lower right-hand corner of Table 3. As can be seen, the use of such a modified heat source module in the MITG would increase the specific power to more than 5 watts per Ib. 
Table 3. Illustrative Design Summary (BOL)

\begin{tabular}{|c|c|c|}
\hline $\begin{array}{l}\text { Heat Source: } \\
\text { Number of GPHS Modules } \\
\text { Thermal Power per Module (w) } \\
\text { BOL. Thermal Power (w) }\end{array}$ & & $\begin{array}{r}12 \\
250 \\
3000\end{array}$ \\
\hline $\begin{array}{l}\text { Thermoelectric Converter: } \\
\text { Number of Thermoelectric Modul } \\
\text { Number of Couples per Module } \\
\text { Leg Width and Thickness (in) } \\
\text { Module Width and Thickness (in) } \\
\text { Leg Length (in) }\end{array}$ & Jles & $\begin{array}{r}96 \\
18 \\
0.057 \\
0.360 \\
0.300\end{array}$ \\
\hline $\begin{array}{l}\text { Generator Dimensions (in): } \\
\text { Housing Side } \\
\text { Heat Source Height } \\
\text { Generator Height } \\
\text { Generator Span }\end{array}$ & & $\begin{array}{r}5.75 \\
25 \\
27 \\
18\end{array}$ \\
\hline $\begin{array}{l}\text { Temperatures PCl: } \\
\text { Fuel Capsule } \\
\text { Heat Source Surface } \\
\text { Hot-Shoe Corner } \\
\text { Hot-Shoe Root } \\
\text { Hot Junction } \\
\text { Cold Junction } \\
\text { Cold Shoe } \\
\text { Housing } \\
\text { Fin Root } \\
\text { Fin Tip }\end{array}$ & $\begin{array}{l}T \\
1269 \\
1100 \\
1096 \\
1020 \\
1000 \\
300 \\
285 \\
270 \\
245 \\
170\end{array}$ & $\begin{array}{r}\Delta T \\
169 \\
4 \\
76 \\
20 \\
700 \\
15 \\
15 \\
25 \\
75\end{array}$ \\
\hline
\end{tabular}

\begin{tabular}{|c|c|}
\hline $\begin{array}{l}\text { Heat Balance (w): } \\
\text { Thermal Power } \\
\text { End Losses } \\
\text { Side Losses } \\
\text { Heat to TE Modules } \\
\text { Heat from TE Modules } \\
\text { Total Heat Rejection }\end{array}$ & $\begin{array}{r}3000 \\
75 \\
100 \\
2825 \\
2543 \\
2718\end{array}$ \\
\hline $\begin{array}{l}\text { Electrical: } \\
\text { Couples in Series } \\
\text { Couples in Parallel } \\
\text { Module Voltage } \\
\text { Module Current (amp) } \\
\text { Generator Voltage } \\
\text { Generator Current (amp) } \\
\text { Power Output (w) }\end{array}$ & $\begin{array}{r}144 \\
12 \\
3.50 \\
0.84 \\
28 \\
10.1 \\
282\end{array}$ \\
\hline $\begin{array}{l}\text { Efficiency (\$): } \\
\text { Thermal (excl. module losses) } \\
\text { Thermoelectric Material } \\
\text { Thermoelectric Module } \\
\text { System }\end{array}$ & $\begin{array}{r}94 \\
10.5 \\
10.0 \\
9.4\end{array}$ \\
\hline $\begin{array}{l}\text { Weight (lbs): } \\
\text { Heat Source } \\
\text { Converter } \\
\text { Total }\end{array}$ & $\begin{array}{l}38.4 \\
21.5 \\
59.9\end{array}$ \\
\hline Specific Power (w/lb): & 4.71 \\
\hline
\end{tabular}

Aeroshell fillets removed, and walls reduced to twice the predicted recession 


\section{RELATIVE CONTRIBUTIONS OF NEW MATERIAL \& NEW DESIGN}

One question of considerable interest is to what extent the specific-power improvement of the MITG is due to the new thermoelectric material, and to what extent it is the result of the new design. This question is addressed in Figure 51, which seeks to separate the two effects. The results are presented in terms of past, present, and future RTGs.

The MHW generator used in the past employed a monolithic MHW heat source and SiGe unicouples; it had a specific power of $1.79 \mathrm{w} / \mathrm{lb}$. The GPHS/RTG presently under construction for the Galileo and Solar Polar missions employs a new modular heat source and the same SiGe unicouples; it has a specific power of $2.34 \mathrm{w} / \mathrm{lb}$. The MITG design described in this paper uses the same heat source modules as the GPHS/RTG, is based on SiGe/GaP multicouples, and employs a substantially different generator design; it has a specific power of $4.71 \mathrm{w} / \mathrm{lb}$.

As shown in the figure, the addition of GaP to SiGe in the present GPHS/RTG design would raise its specific power to $3.02 \mathrm{w} / \mathrm{lb}$; conversely, the use of the MITG design with the present SiGe material would result in a specific power of $3.42 \mathrm{w} / \mathrm{lb}$. Thus, it is clear that the new material and the new design both make important contributions to the improved specific power of the MITG. In fact, it appears that slightly more of the improvement is attributable to the new design than to the new material.

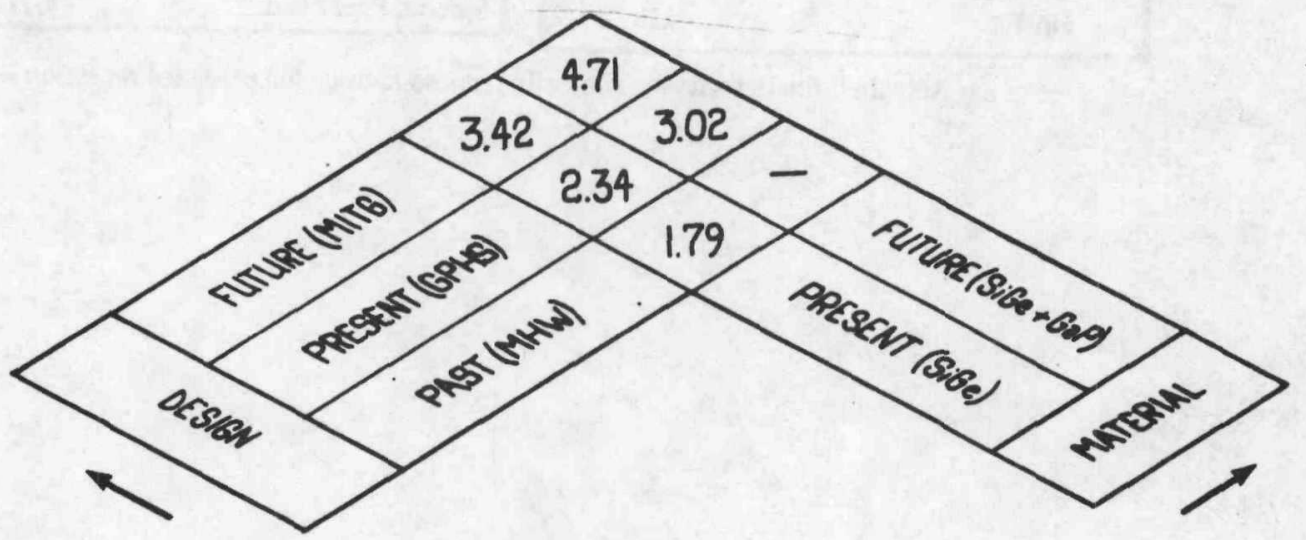

Figure 51. RTG Specific-Power Improvement (Watt/Lb) 


\section{CONCLUSION}

The MITG design promises a substantially higher specific power than present-generation RTGs. This is achieved without any reduction in safety, and with probably increased reliability, because of the ability to check the performance of individual thermoelectric modules in the assembled converter, and the ability-if necessary - to replace those which are deficient. Moreover, in the long run the MITG should be more economical, because of its modularity and scalability, and because of its greater flexibility in matching the payload's voltage requirements.

It is recognized, of course, that it is risky to compare the weight of a conceptual design with that of a mature design that is actually under construction. The GPHS/RTG design has been worked out in full detail, and its weight is unlikely to change much. Although care was taken in assessing the MITG design to make certain that no parts were overlooked and to prepare realistic weight estimates for all parts, it is of course possible that its weight will grow as the result of unforeseen problems or difficulties during further development. Nevertheless, the weight advantage of the MITG is so great that there is a strong incentive for exploring this approach. DOE is therefore planning a series of tests, each consisting of a complete (electrically heated) MITG slice, to assess the feasibility and performance of that design. 


\section{Acknowledgement}

The author takes pleasure in acknowledging the valuable contributions of V. Raag of Syncal; F. Huffman, S. Matsuda, and G. Miscolezy of Thermoelectron; and T. Kline, H. Sookiazian, M. Mukunda, and E. Vogel of Fairchild.

\section{References: (Part A):}

[1] G.L. Bennett; J.J. Lombardo; B.J. Rock, "Space Nuclear Electric Power Systems", 1980 Annual Meeting of American Astronautical Society (Paper 80-220), to be published in Volume 44 of "Advances in the Astronautical Sciences".

[2] A.A. Pitrolo; R.B. Morrow; A.J. Arker, "Multi-Hundred Watt Radioisotope Thermoelectric Generator (MHW-RTG)", 1971 IECEC (Intersociety Energy Conversion Engineering Conference), Paper No. 719069, August 1971, Pg. 492.

[3] C.E. Kelly, "The MHW Converter (RTG)", 1975 IECEC, Paper No. 759132, August 1975, Pg. 880.

[4] L. Garvey; G. Stapfer, "Performance Testing of Thermoelectric Generators Including Voyager and LES 8/9 Flight Results", 1979 IECEC, Volume II, Paper No. 799312, August 1979, Pg. 1470.

[5] A. Schock, "Design Evolution and Verification of the General-Purpose Heat Source", 1980 IECEC, Volume II, Paper No. 809203, August 1980, Pg. 1032.

[6] A. Schock; H. Sookiazian, "Design Optimization of RTG for Solar-Polar Mission", 1979 IECEC, Volume II, Paper No. 799307, August 1979, Pg. 1444.

[7] R.D. Cockfield; R.F. Hartman; C.E. Kelly, "RTG Power Sources for the International Solar Polar Mission", 1980 IECEC, Volume 2, Paper No. 809204, August 1980, Pg. 1043.

[8] Michael L. Paquin, "The Multi-Foil Thermal Insulation Development Program", 1969 IECEC, Paper No. 699047, September 1969, Pg. 408.

[9] R.R. Heikes; R.W. Ure, "Thermoelectricity: Science and Engineering", Interscience Publishers, N.Y. 1961, Section 15.6.4.

[10] Ibid, Section 15.4.1,2.

[11] G. Stapfer; V.C. Truscello, "Sublimation Behavior of Silicon Nitride $\left(\mathrm{Si}_{3} \mathrm{~N}_{4}\right)$ Coated Silicon Germanium (SiGe) Unicouples", 1975 IECEC, Paper No. 759110, August 1975, Pg. 730.

[12] F.N. Huffman; S. Matsuuda; G. Miskolczy; C. Wang, "Meltdown Characteristics of Multi-Foil Thermal Insulation", 1979 IECEC, Volume II, Paper No. 799298, August 1979, Pg. 1390.

[13] A. Schock; A. Shostak, "Use of Modular Heat Source Stack in RTGs", 1979 IECEC, Volume II, Paper No. 799305, August 1979, Pg. 1432.

[14] A. Shostak; A. Schock, "Dynamic Response of an RTG Cantilevered from a Shuttle-Launched Spacecraft", AIAA/ASME 21st SDM Conference, Seattle, Washington, May 1980. 
Part B.

\title{
MITG Test Assembly Design and Fabrication*
}

\author{
A. Schock \\ Fairchild Space Company \\ Germantown, Maryland 20874
}

(Presented at the 1983 Intersociety Energy Conversion Engineering Conference)

\section{Abstract}

The design, analysis, and evaluation of the Modular Isotopic Thermoelectric Generator (MITG), described in an earlier paper, led to a program to build and test prototypical modules of that generator. Each test module duplicates the thermoelectric converters, thermal insulation, housing and radiator fins of a typical generator slice, and simulates its isotope heat source module by means of an electrical heater encased in a prototypical graphite box. Once the $\sim 20$-watt MITG module has been developed, it can be assembled in appropriate number to form a generator design yielding the desired power output.

The present paper describes the design and fabrication of the MITG test assembly, which confirmed the fabricability of the multicouples and interleaved multifoil insulation called for by the design. Test plans, procedures, instrumentation, results, and posttest analyses, as well as revised designs, fabrication procedures, and performance estimates, are described in subsequent papers in these proceedings. 


\section{Introduction}

At the 1981 Intersociety Energy Conversion Engineering Conference (IECEC), the author presented a paper [1] describing the conceptual design and analysis of an advanced Radioisotope Thermoelectric Generator (RTG). The design was designated as the Modular Isotopic Thermoelectric Generator (MITG), because of its modularity. Except for end sections, the generator is composed of identical slices, each producing over 20 watts at 28 volts. A typical generator, containing twelve such slices, is depicted in Figure 1, which shows the overall dimensions of the unit.

Once the basic generator slice has been developed, a 28-volt generator design of any required power level can be readily assembled by inserting an appropriate number of modular siices between the end sections. required for containment, thermal insulation, and heat source support. This modularity essentially eliminates the need for developing a custom-made generator for each new mission. 
Figure 1. MITG Design Illustrative Example: 12 Slices, 246 Watt, 59 Lb

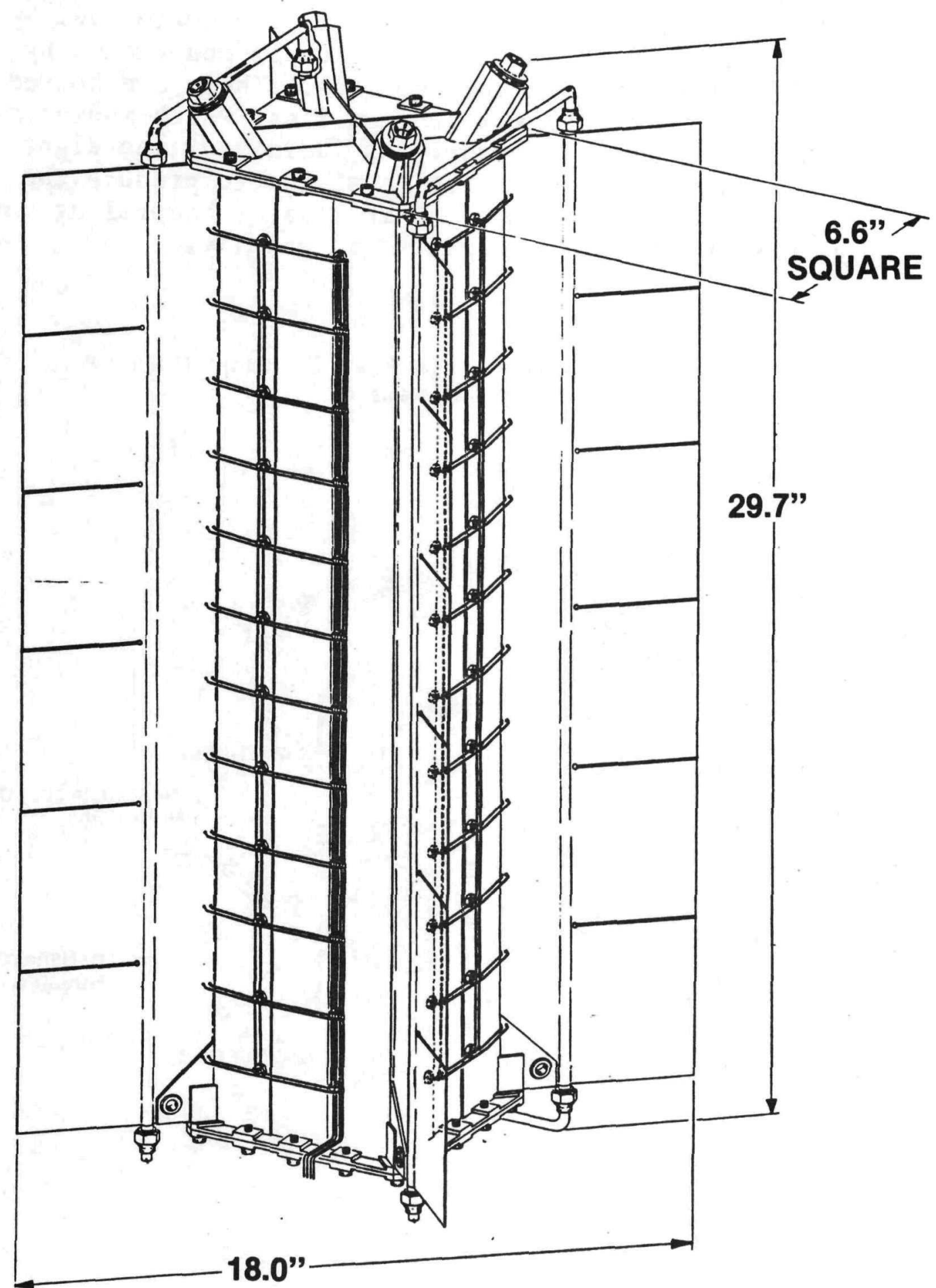


The design of a typical generator slice is illustrated in Figure 2. Each slice contains one Genera1Purpose Heat Source (GPHS) module [2], surrounded by multifoil thermal insulation which is penetrated by eight thermoelectric multicouples. These are bolted to a square housing with corner fins, which radiates their waste heat to space. As indicated, the eight multicouples are connected in series to produce the desired 28-volt output, and the slices comprising the generator are cross-connected in parallel.

Figure 2. Typical Generator Slice (20.5 watts at 28 volts) Split at Mid-Plane, to Display Internal Components

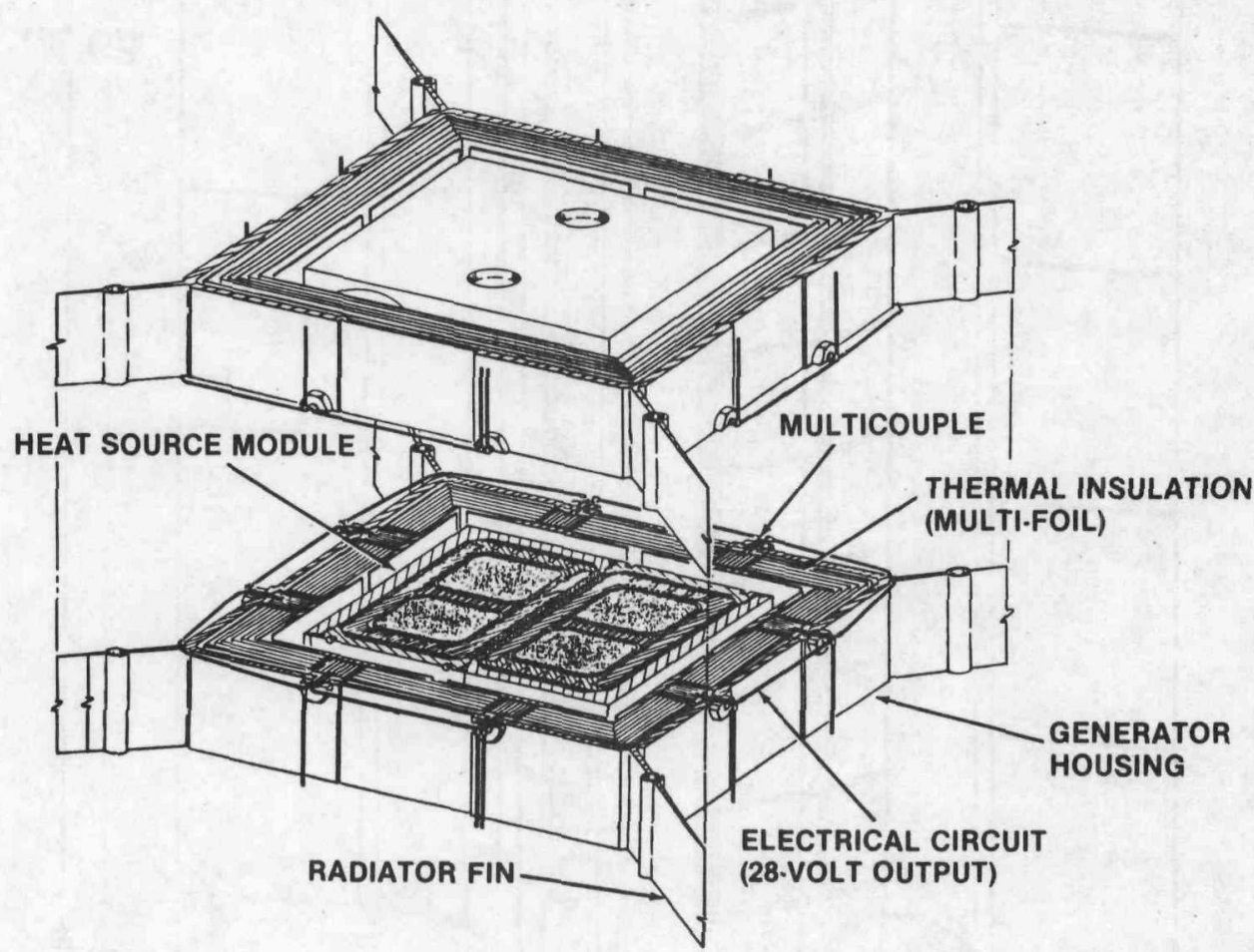


The heat source module has a beginning-of-life thermal power of 250 watts, produced by alpha-decay of four iridium-clad $\mathrm{Pu}^{238_{0}} \mathrm{O}_{2}$ fuel capsules. It also contains various graphite components designed to prevent fuel dispersal in all plausible accidents.

As can be seen, the heat source module does not touch the thermoelectric multicouples. Heat transfer between them is by radiation only. The multicouple, whose design is described in detail in Section 4, consists of 18 or 20 series-connected thermoelectric couples. The thermoelectric material used is SiGe with a GaP additive. SiGe has been successfully used on a number of previous space flights. The GaP additive significantly improves the performance of the base material, by reducing its thermal conductivity [1]. The geometric relation between the heat source module 'and the surrounding multicouples is shown more clearly in Figure 3.

Figure 3. Heat Source Module Surrounded by Eight Multicouples

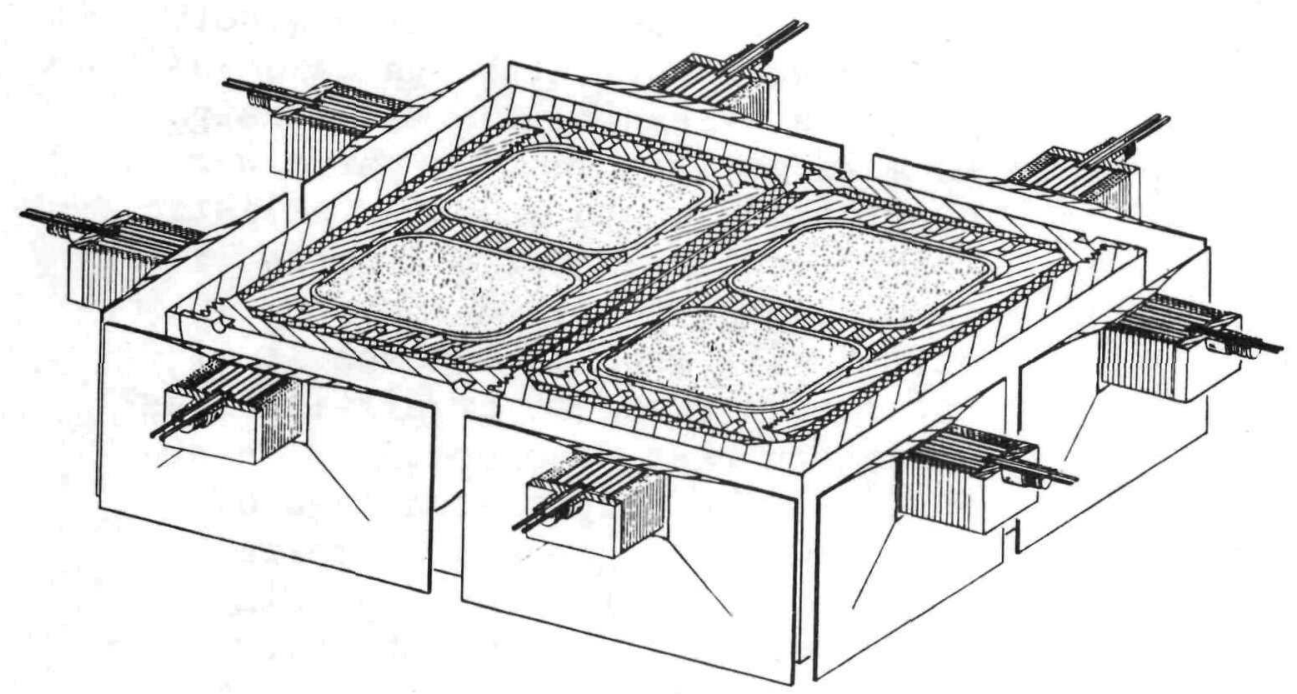


The author's 1981 paper concluded with the following two paragraphs:

"The MITG design promises a substantially higher specific power than the present-generation GPHS/ RTG. This is achieved without any reduction in safety; and with probably increased reliability, because of the ability to check the performance of individual multicouples in the assembled converter, and the ability - if necessary - to replace those which are deficient. Moreover, in the long run the MITG should be more economical, because of its modularity and scalability, and because of its greater flexibility in matching the payload's voltage requirements.

It is recognized, of course, that it is risky to compare the weight of a conceptual design with that of a mature design that is actually under construction. The GPHS/RTG design has been worked out in full detail, and its weight is unlikely to change much. Although care was taken in assessing the MITG design to make certain that no parts were overlooked and to prepare realistic weight estimates for all parts, it is of course possible that its weight will grow as the result of unforeseen problems or difficulties during further development.. Nevertheless, the weight advantage of the MITG is so great that there is a strong incentive for exploring this approach. DOE is therefore planning a series of tests, each consisting of a complete (electrically heated) MITG slice, to assess the feasibility and performance of that design." 
Subsequently, Fairchild was asked to design and prepare detailed drawings of the test assembly; Syncal received a contract to develop and build the multicouples and generator housing; and Thermo Electron received a Syncal subcontract for the electrical heaters and the multifoil thermal insulation*. In addition, Fairchild was asked to prepare a detailed test plan, to assemble the required test facilities, instrumentation, and computer hardware and software, and to carry out the planned tests. After the initial tests, Fairchild participated in post-test examination of the hardware, and performed additional analyses and design improvements.

The present paper describes the objectives of the above tests, and the design and fabrication of the test assemblies. The next paper in these proceedings [3] describes the test plan, test methodology, instrumentation, computer-controlled test operation and data acquisition, and the initial test results. The following paper [4] describes the post-test analysis, including a very detailed thermal-stress analysis to identify the cause of the observed problems. Based on the lessons learned in these analyses, the paper describes a number of design changes to eliminate the thermalstress problem, and presents analyses which demonstrate their effectiveness.

Finally, the last paper in this series [5] examines a number of additional design modifications to eliminate the observed problems, leading to a revised multicouple design. These modifications have been validated analytically, and are now undergoing experimental evaluation. Based on the revised design and on the latest thermoelectric property measurements, that paper presents updated weight and performance predictions for typical MITG flight generators, and compares their power-toweight ratios to current RTGs and to the 1981 MITG prediction.

* Note: Syncal, headed by Dr. V. Raag, merged with Thermo Electron in the summer of 1982, and is now part of that company. 
2. Test Objectives below:

The principal MITG test objectives are listed

- Measure power output and system efficiency of a prototypical MITG slice at design temperature.

- Measure effect of GaP additive on the electrical and thermal performance of SiGe multicouples in a realistic generator environment.

- Measure long-term performance stability of prototypical MITG slices, with and without GaP additive.

- Measure reproducibility of individual multicouples and variability of their long-term performance stability in MITG slice.

- Determine effectiveness of glass coating in suppressing Si sublimation, and perform measurements necessary for predicting longterm shunt degradation effects in generator.

- Measure effect of 1 aunch vibration on cantilevered multicouples supporting multifoil insulation.

- Measure effect of heat rejection temperature on MITG performance, to aid design optimization. 


\section{Overview of Test Assembly Design}

The MITG test assembly is depicted in Figure 4. As can be seen, each test assembly consists of a prototypical MITG slice, plus end sections for thermal insulation. At the center of the assembly is a graphiteencased electrical heater, whose outer dimensions duplicate those of the isotopic GPHS module. The assembly housing is insulated by multifoil thermal insulation, with appropriate penetrations for the eight prototypical multicouples.

Heat rejected by the multicouples flows through the housing wall to the corner fins. The fins are water-cooled rather than radiation-cooled, to permit calorimetric measurements. Separate cooling loops are provided for the prototypic side walls and for the nonprototypic end covers. This will aid in predicting the efficiency of a multi-slice generator. There is no metal-to-metal contact between the side walls and the end covers. To minimize heat losses to the vacuum chamber walls, the entire assembly was enclosed in Min-K thermal insulation.

Figure 4. MITG Test Assembly

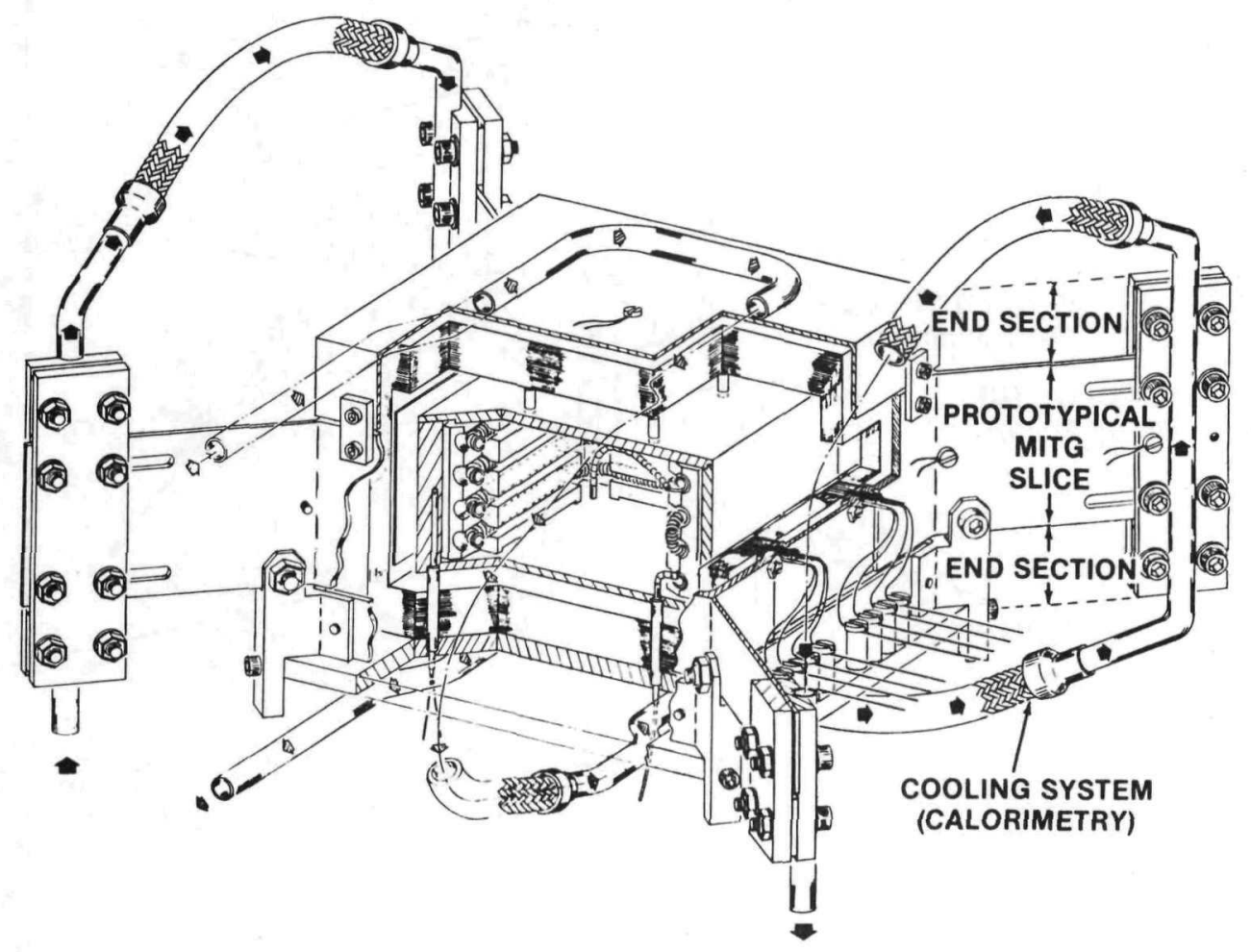




\section{Multicouple Design and Fabrication}

Figure 5 presents an exploded view of the multicouple, reproduced from the 1981 paper [1]. The figure also illustrates the changes in nomenclature agreed to by the various program participants since then. It shows the current component designations, together with the superseded 1981 designations in parentheses.

Figure 5. Exploded View of Multicouple (Thermoelectric Module)

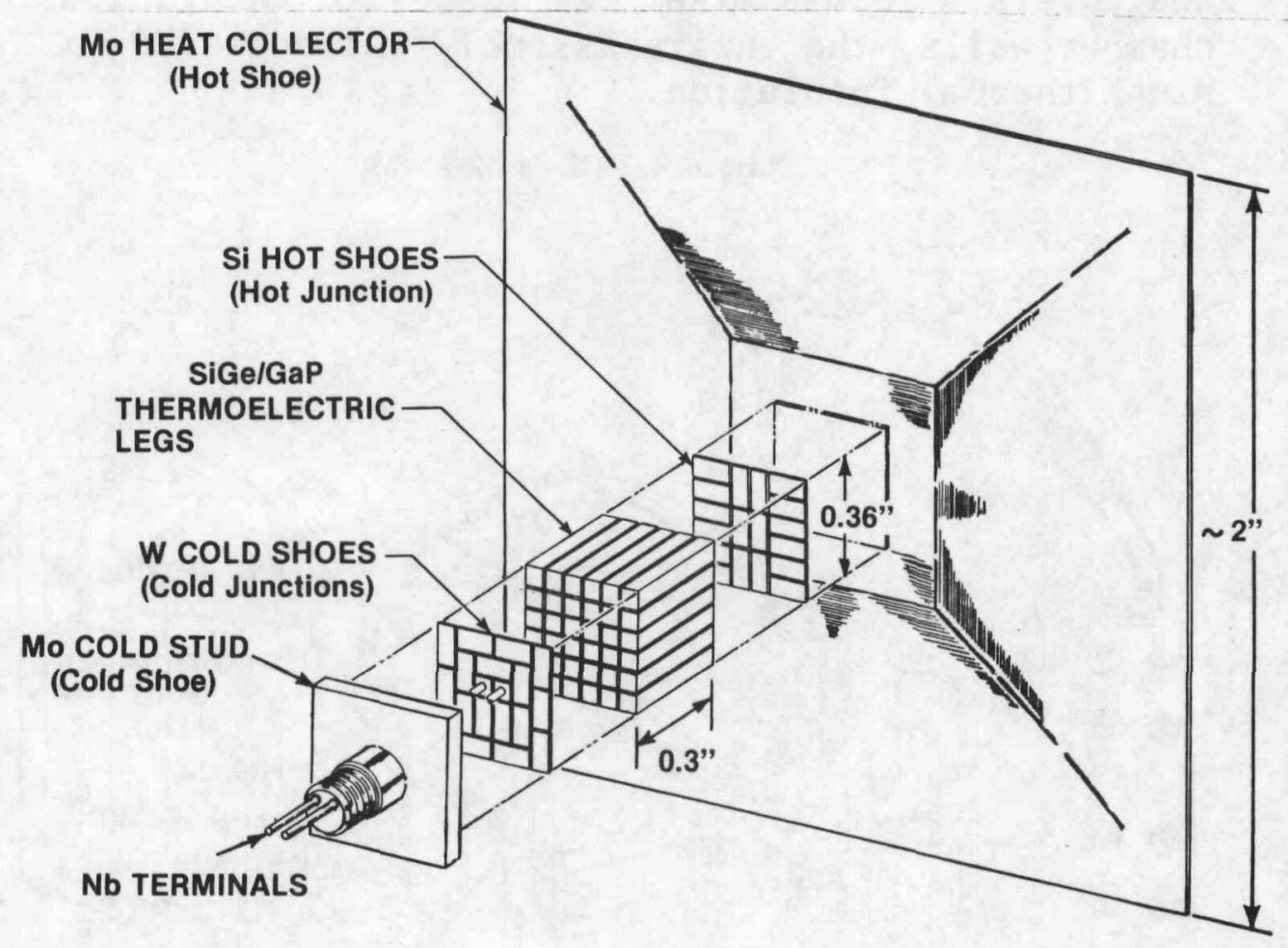


Figure 6 illustrates the design of a typical multicouple used in the initial test assemblies. As can be seen, it was very similar to the design shown in Figure 5, except for the addition of $\mathrm{W} / \mathrm{Nb}$ thermocouples to measure the hot-end and cold-end temperatures; and for the minor changes in the heat collector, hot shoes, and cold studs discussed below.

Figure 6. Multicouple Design for Initial MITG Tests

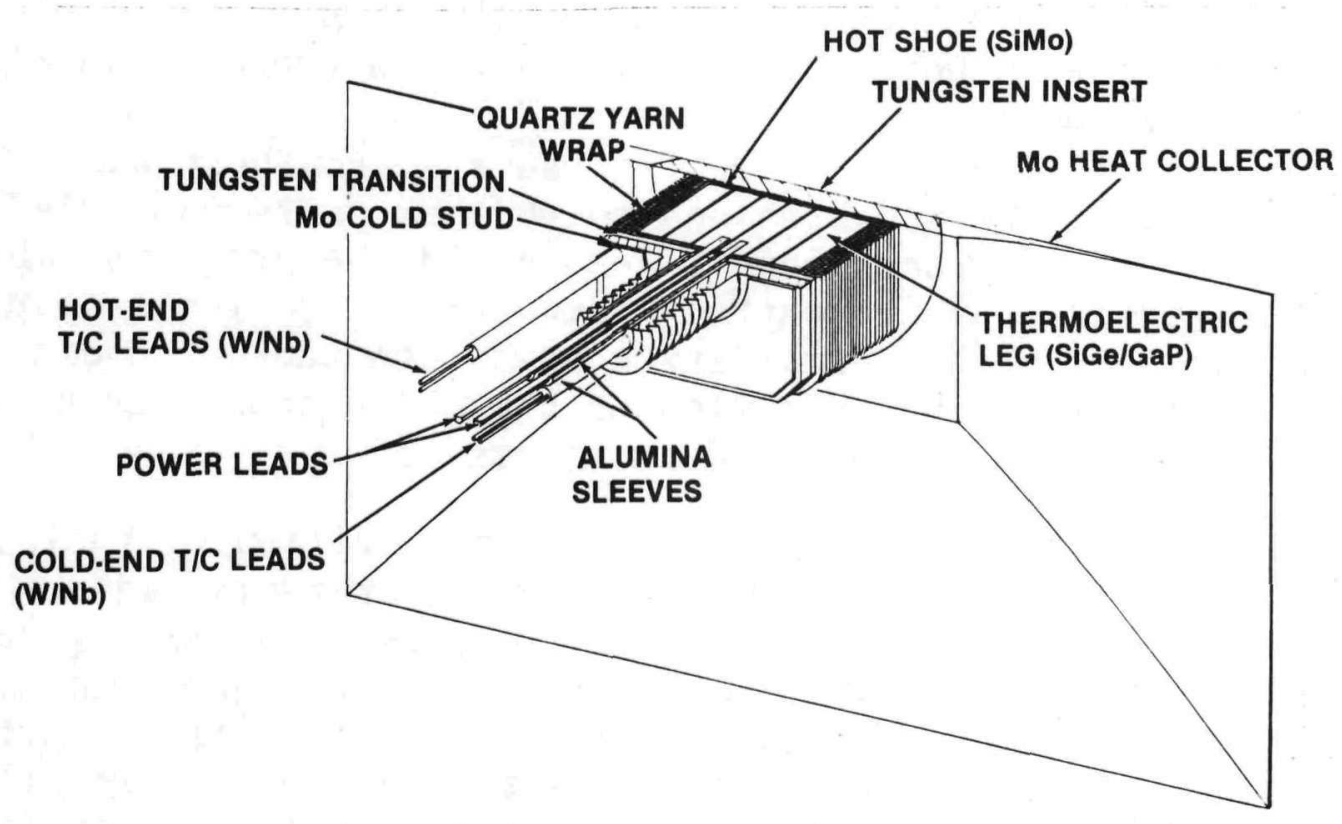


Figure 7 shows an exploded view of the principal multicouple components. The heat collector and cold stud differ somewhat from the all-molybdenum designs described in Reference [1]. It was felt by Syncal that the use of molybdenum components bonded to the SiGe legs was too risky, because of the difference in thermal expansion coefficients. On the other hand, tungsten - which is a much better match - was considered too heavy for the heat collector, and too hard to machine for the threaded cold stud. This resulted in the choice of bimetallic moly-tungsten components. This bimetallic construction caused no fabrication problems in the case of the cold stud, but considerable problems in the case of the heat collector.

When the conical tungsten insert was joined to the molybdenum heat collector by a high-temperature furnace braze, the resultant assembly appeared to be smooth and stress-free, without distortions. But the prolonged exposure to the hot braze furnace was found to have completely embrittled the thin edges of the molybdenum heat collector, which was therefore deemed to be unusable.

To avoid this problem, an electron-beam weld between the tungsten and molybdenum parts was tried. This fast process did indeed avoid the previous embrittlement, but its extreme temperature gradients resulted in very high thermal stresses, which caused severe wrinkling and distortion of the molybdenum heat collector, as illustrated in Figure 8a.

Fairchild then suggested the addition of radial stress relief slots at $45^{\circ}$ or $90^{\circ}$ intervals, which largely eliminated the wrinkling, as shown in Figure 8b. The resultant heat collectors were not perfect, but were good enough to proceed with the initial sets of multicouples. However, these heat collectors still had locked-in stresses, resulting in some edge curling during subsequent testing.

The above problem has now been completely eliminated by the use of a graphite heat collector, as suggested by Thermo Electron. This design change, which is discussed in Reference [4], has been validated and adopted in current production. 
Figure 7. Principal Multicouple Components

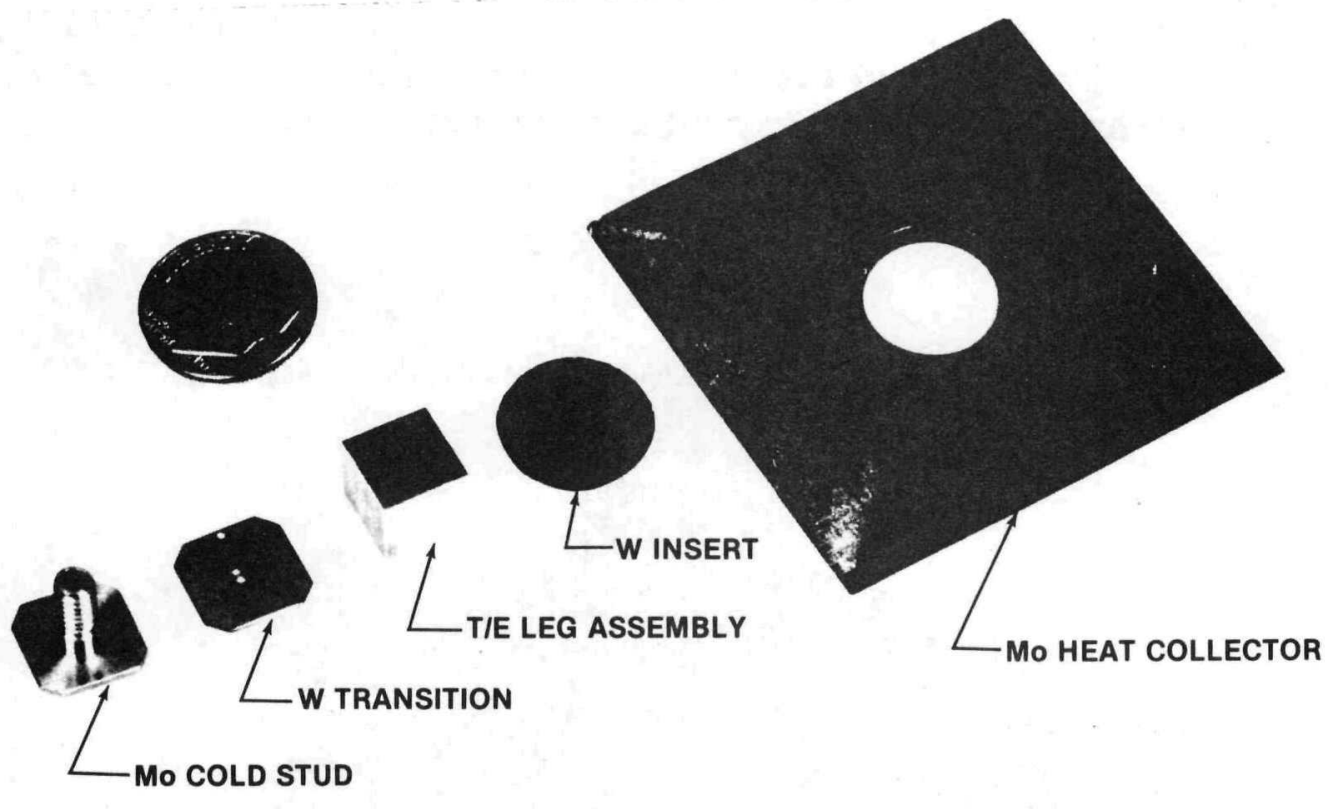

Figure 8. Effect of Stress-Relief Slots on EB-Welded W/Mo Heat Collector

$8 a$

$8 b$

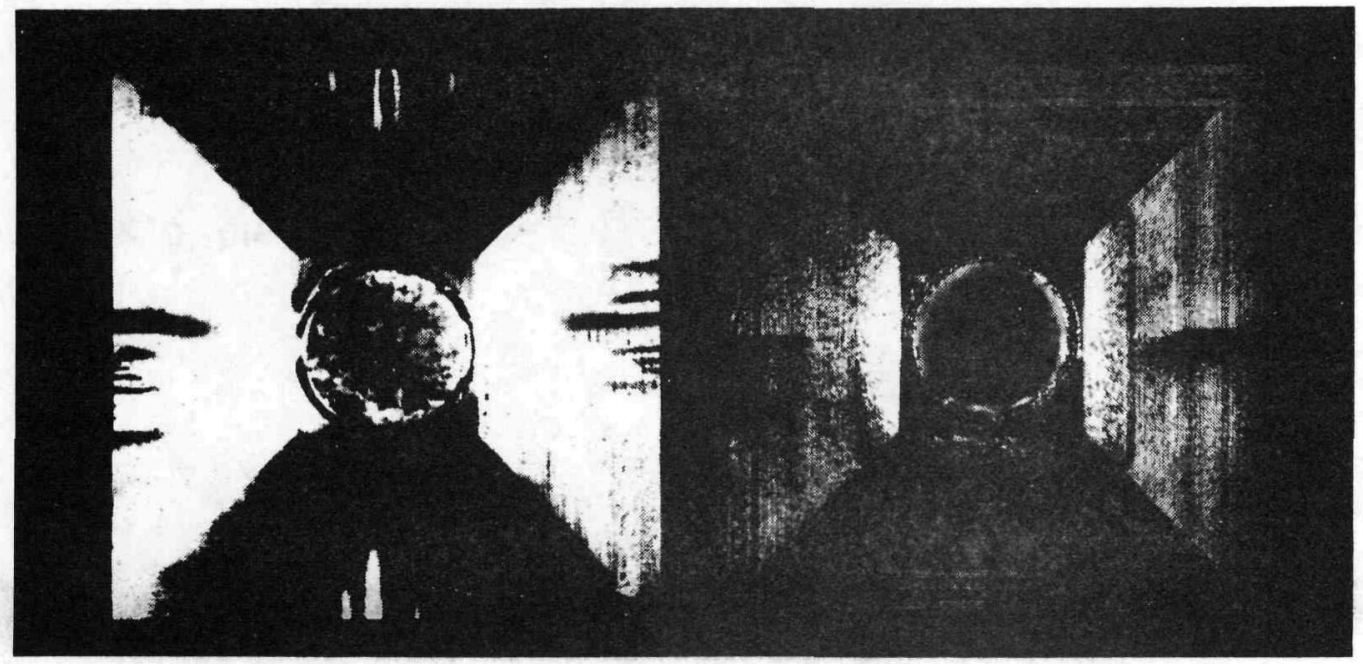


Fabrication of the thermoelectric leg assembly by Syncal proceeded as planned. Figure 9 shows the thirty six $0.057 " \mathrm{\prime} \times 0.057 "$ legs, bonded together by $0.002^{\prime \prime}$ thick glass layers, with the $\mathrm{n}$ - and p-legs arranged in a checkerboard pattern.

Figure 9. Glass-Bonded Thermoelectric Leg Assembly

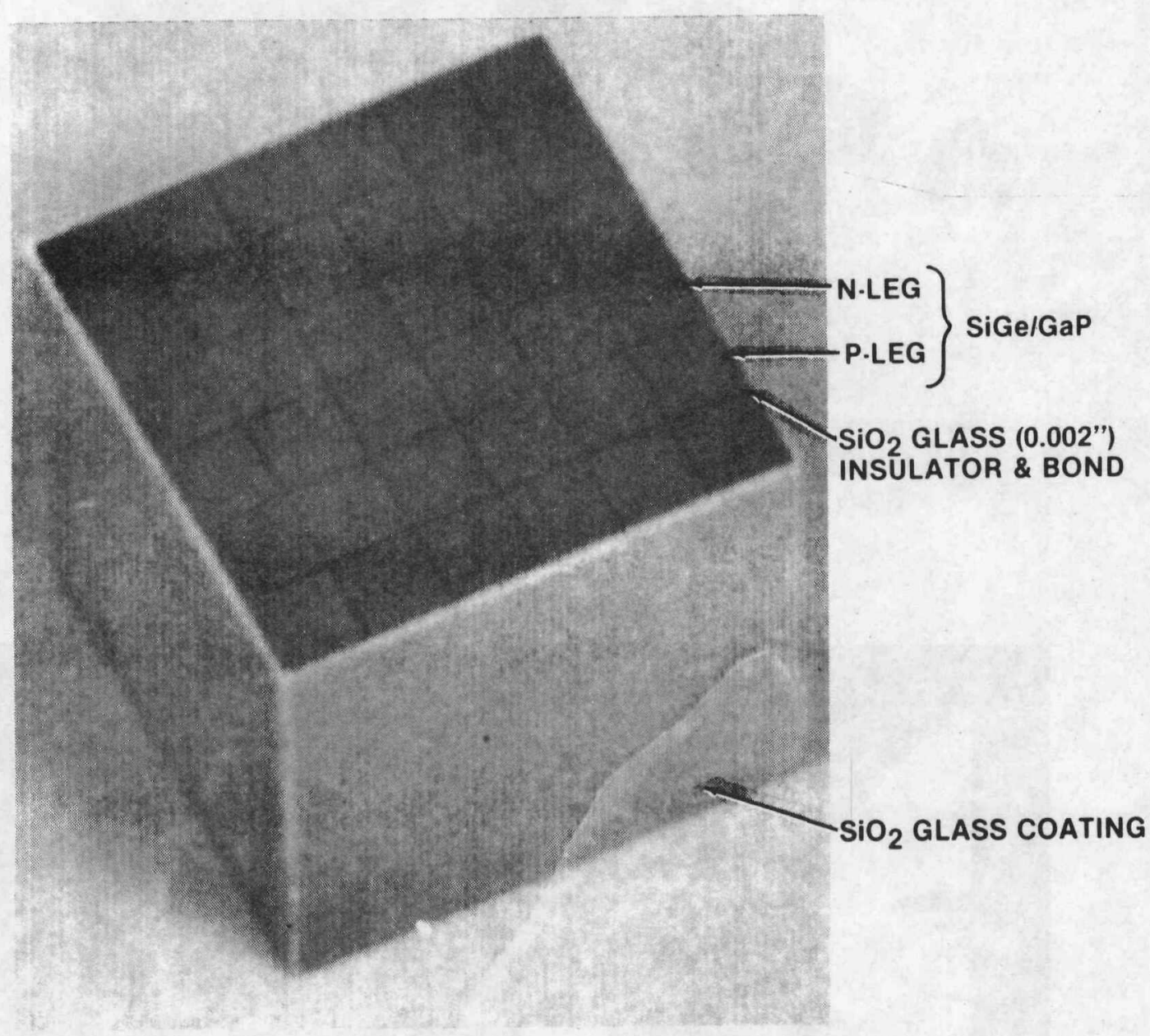


Figure 10 shows a top view of the leg assembly, after addition of the hot shoes, which were diffusionbonded by means of a thin sputtered germanium layer. The 1981 design had called for $0.008^{\prime \prime}$-thick silicon hot shoes. Subsequently, it was concluded that such hot shoes would result in excessive hot-end voltage drops. To avoid these, three design changes were instituted: the hot-shoe material was changed from $\mathrm{Si}$ to SiMo $(85 / 15)$, to improve the electrical conductivity; the hot-shoe thickness was increased from 0.008 " to 0.030 ", and the cold-shoe thickness was increased from 8 microns to 18 microns; and the multicouple leg arrangement was completely reconfigured to increase the conductance of the hot-end connectors. These three changes greatly reduce the multicouple's hot-end voltage drops.

Figure 10. SiMo Hot Shoes Diffusion-Bonded to Ends of SiGe Legs

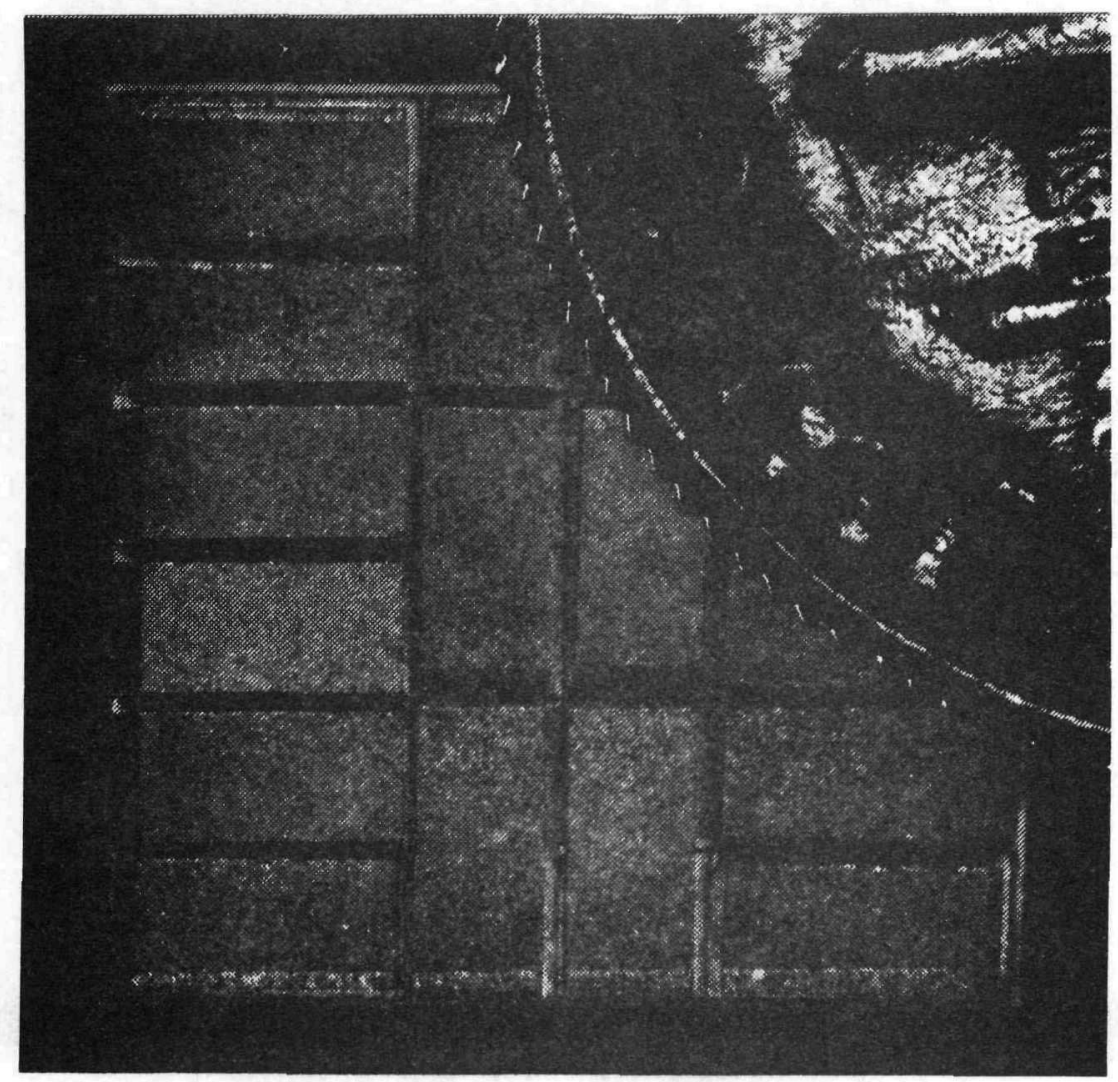


The change from Si to SiMo was validated by high-temperature compatibility tests at Thermo Electron. The effect of thickening the hot shoes was analyzed in detail by Fairchild. A two- or three-dimensional electrical analysis had to be employed, because the current redistributes itself to find the path of least resistance. This results in a highly non-uniform current density, as illustrated by the typical solution presented in Figure 11, showing isopotential lines and current flow paths.

Figure 11. Typical Current Distribution at Hot End of Couple

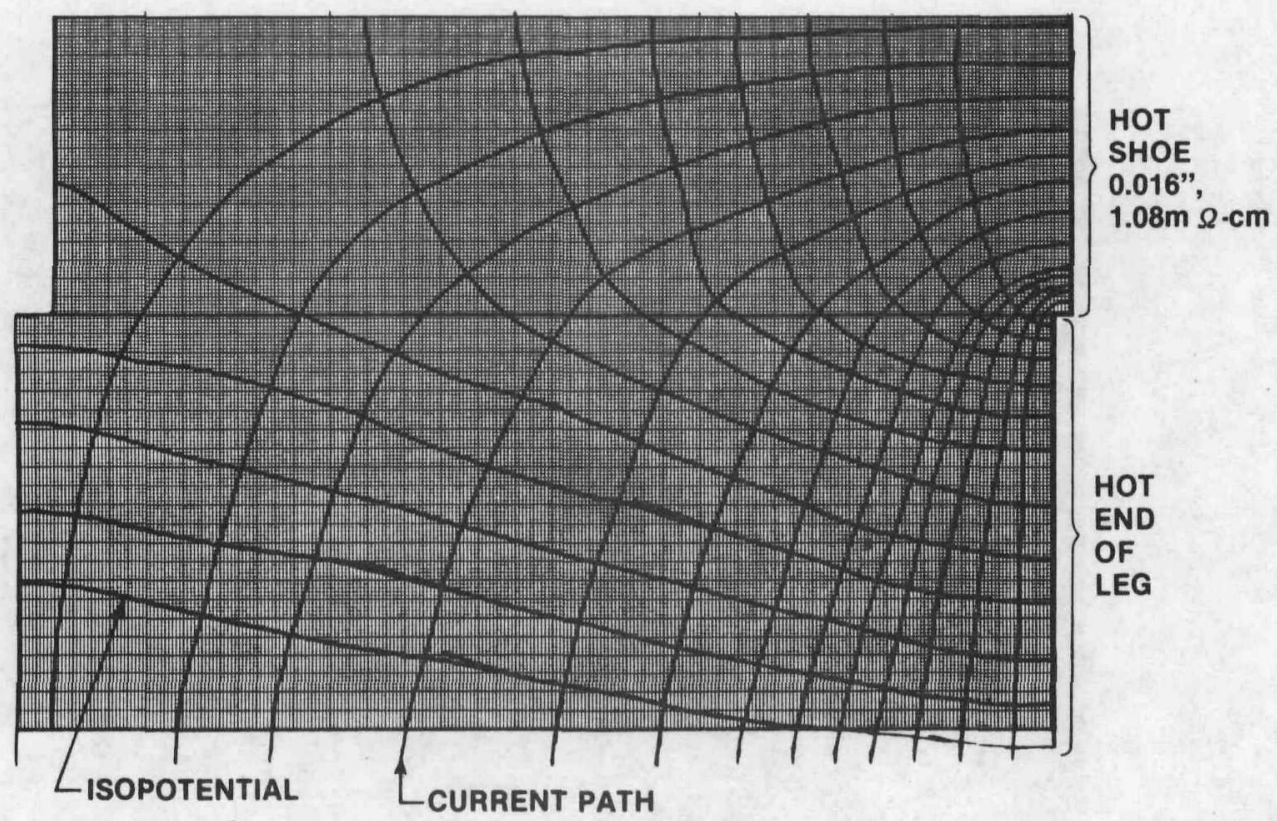


The effect of hot- and cold-shoe thickness on couple efficiency is shown in Figure 12. As can be seen, doubling or tripling the thickness of the original 0.008 " hot shoe was very effective in reducing its resistance. But increasing the thickness beyond 0.030" offers relatively little benefit, because the resistance reduction in the lateral direction is counteracted by the resistance increase in the axial direction. (The results shown are for an obsolete thermoelectric leg arrangement and for superseded 1980 material properties. For the current materials and design, the absolute values of efficiency are different, but the trends would be the same.)

The figure also illustrates that there is a similar point of diminishing returns with respect to the cold-shoe thickness.

Figure 12. Effect of Hot- and Cold-Shoe Thickness on Couple Efficiency

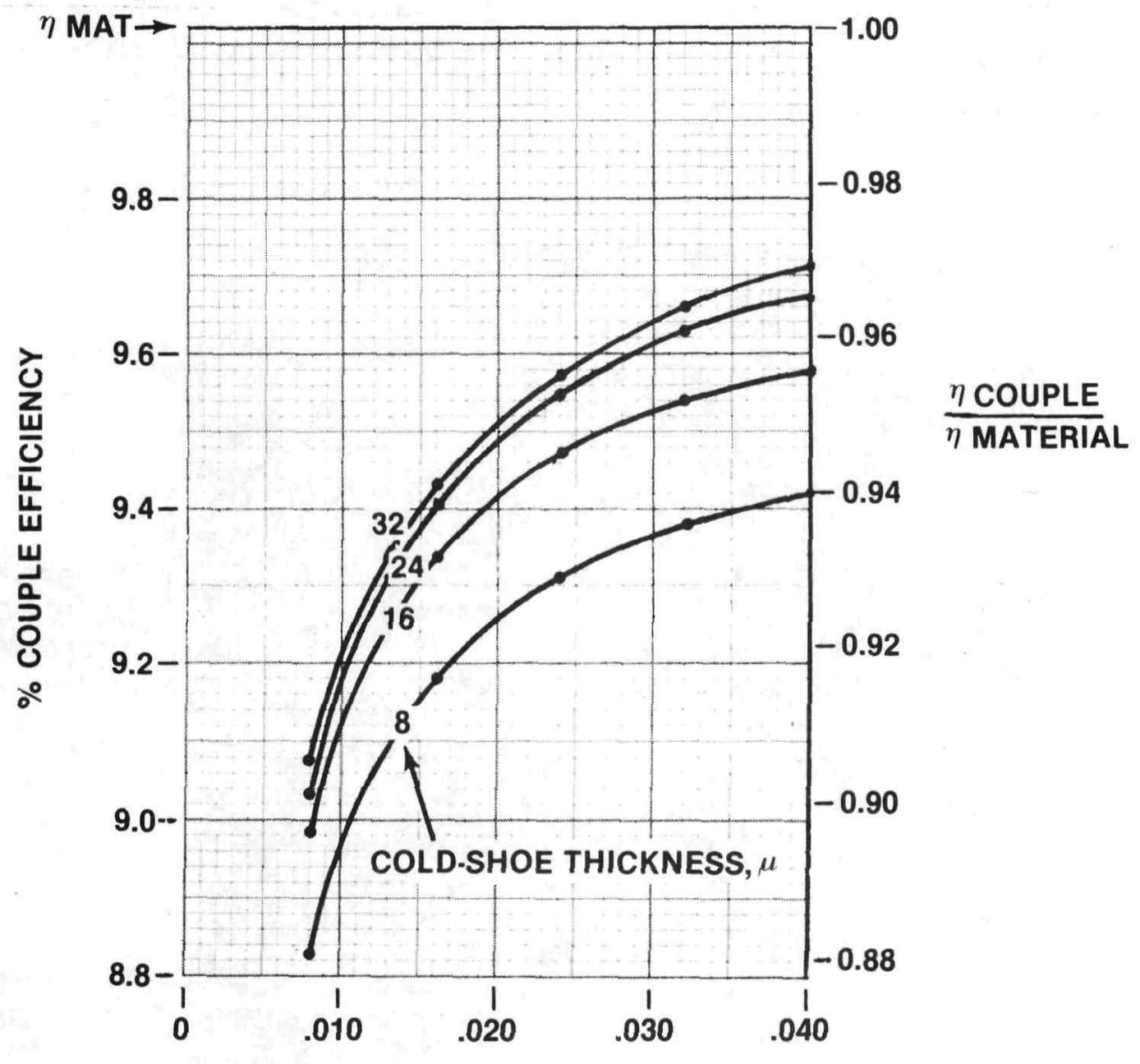

HOT SHOE THICKNESS, Inch 
The material and thickness changes discussed above were incorporated in the initial test assemblies. The third design change, which required a reconfiguration of the multicouple leg arrangement, could not be implemented in time for those assemblies. It is included in the multicouples currently being fabricated for the next test assembly. That design change, which also involves a significant change in fabrication procedure, is described and discussed in Reference [5], Section 3.2.

After the thermoelectric leg assemblies were glass-bonded to the heat collectors, their cold ends were sputter-coated with a 20 -micron tungsten layer to form the cold shoes. The required gaps between cold shoes were produced by photo-etching, using a photographic mask that matches the cold-shoe pattern shown in Figure 5. The results of this process are illustrated in Figure 13. Recently, the tungsten sputtering step has been replaced by chemical vapor deposition. This makes it practical to apply a thicker deposit $(0.001 ")$, which reduces the cold-shoe's electrical resistance to negligible levels.

Figure 13. Sputtered and Photo-Etched Cold Shoes

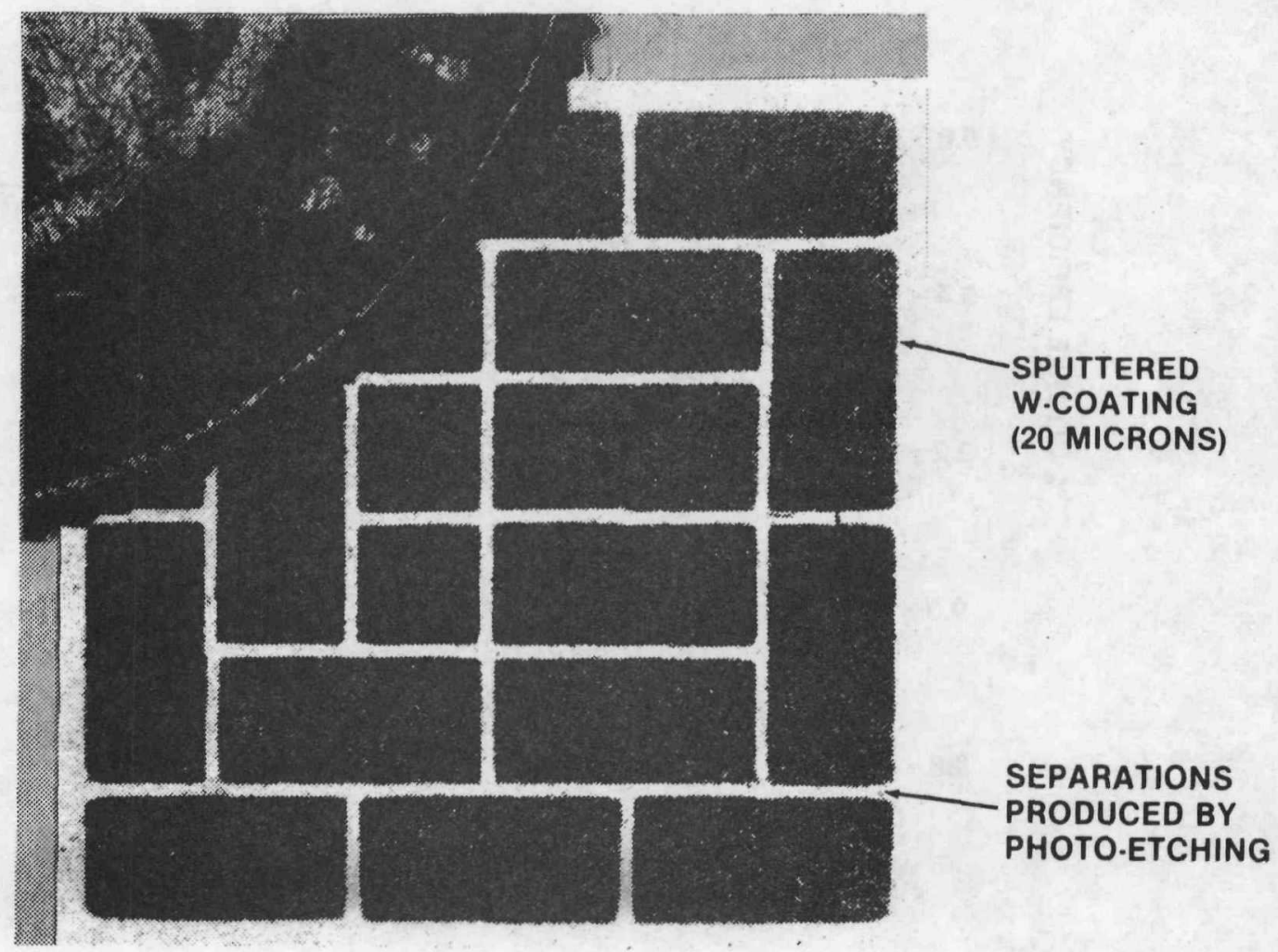


After deposition of the cold shoes and a covering glass layer, 0.060"-deep holes were drilled into the two central legs to facilitate brazing of the lead wires (See Figure 5). Nickel wires with a silveraluminum braze alloy were used. This procedure has since changed, as discussed in Reference [5], Section 3.3.

The thermopile assembly was then bonded to the tungsten cover of the bimetallic cold stud. A lowmelting $\left(680^{\circ} \mathrm{C}\right)$ glass was used as the bonding agent, to avoid adverse reactions between the tungsten cold shoes and the SiGe legs. A photograph of a completed multicouple, without the quartz yarn wrap, is shown in Figure 14. The multicouple shown is after a test of several hundred hours. It shows the curling of the heat collector's thin edges that was mentioned earlier.

Figure 14. Completed Multicouple (After Test)

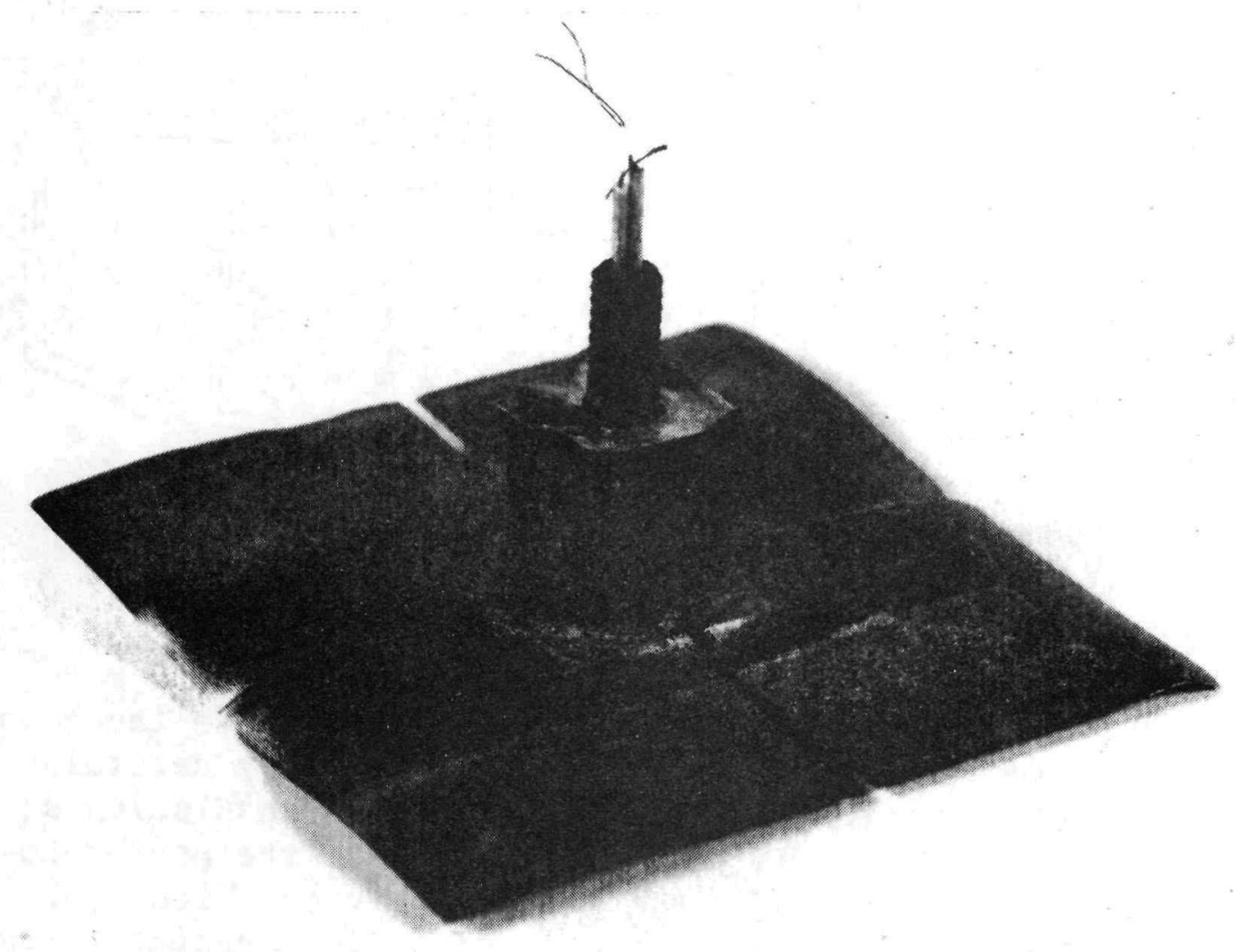




\section{Test Housing and Insulation Design}

Figure 15 depicts the side housing, base plate, and cooling loops of the test assembly. (This and subsequent figures are somewhat misleading, since the cooling loop is actually the last item added to the test assembly.) The positions of the cooling loop's copper saddle plates on the housing fins are adjustable, to make it possible to vary the thermal resistance to the heat sink. As mentioned earlier, the base plate has a separate coolant loop. Note that the side frame and base plate do not touch. To minimize heat transfer, they are connected through ceramic bushings, to avoid metal-to-metal contact.

Figure 15. Test Assembly Housing and Cooling Loops

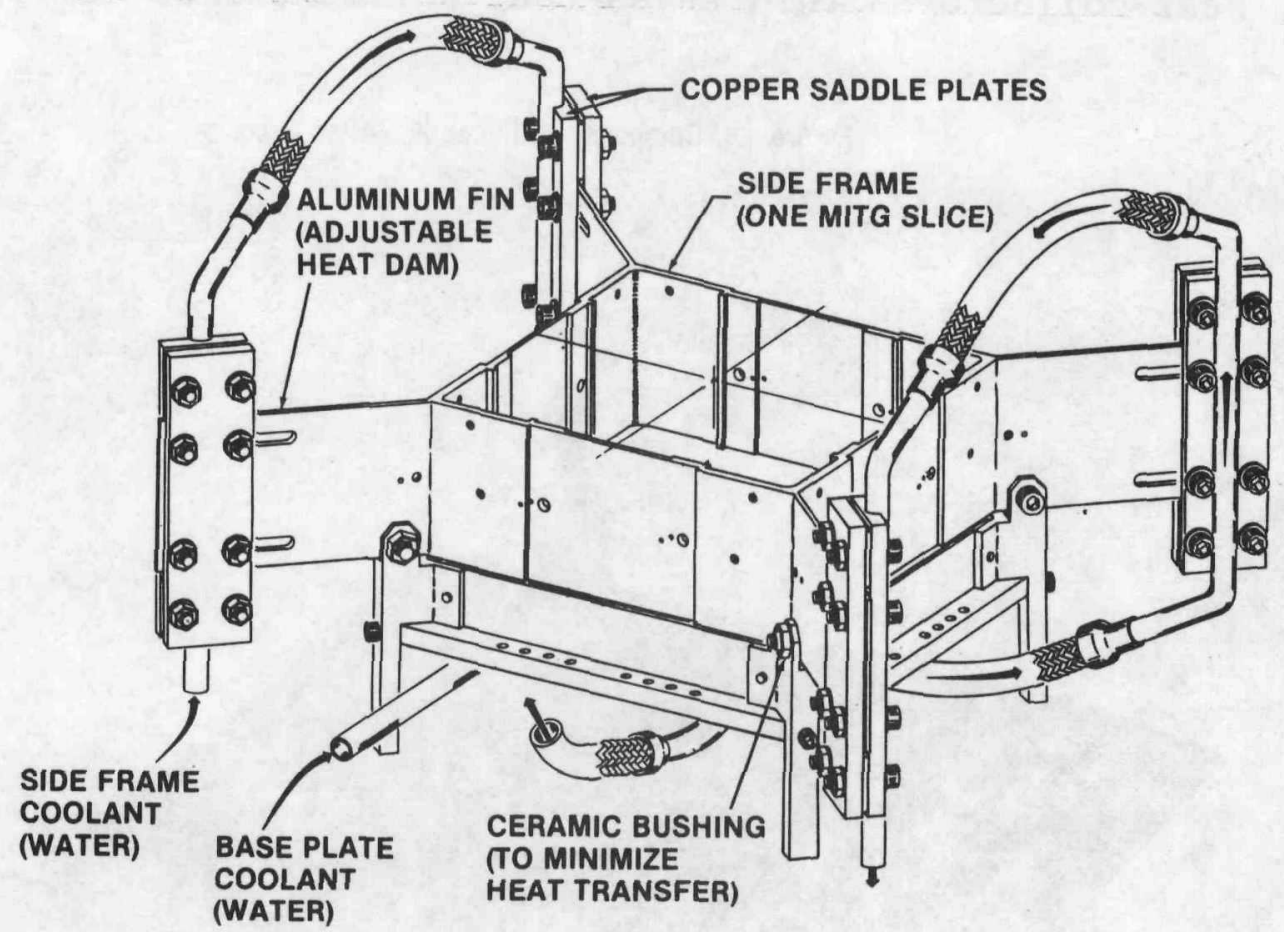

The multifoil thermal insulation inside the housing is a critical item for accurate efficiency determinations. It must simulate the MITG side insulation as closely as possible, but must minimize the non-prototypic heat losses to the two end covers, which could otherwise be dominant in a single-slice generator test. Particular care must be taken to minimize edge losses and corner losses, and also thermal losses through thermocouple penetrations and heater leads. 
The insulation system design used in the MITG tests is illustrated in Figure 16. The multifoil assembly consists of 0.0003 -inch thick molybdenum foils separated by zirconia particles covering about $6 \%$ of the foils' surface. Each side wall is insulated by 60 layers, as in the flight system design, and each end cover by 120 layers. The foils are separated by silica paper washers at each multicouple penetration, to prevent compression during multicouple insertion. As indicated, there are interleaved foil overlaps at all six edges. To minimize heat losses, there are only four small thermocouple and heater lead penetrations, as shown at the bottom of the foil package, and none at the top.

Figure 16. Interleaved Multifoil Insulation Assembly

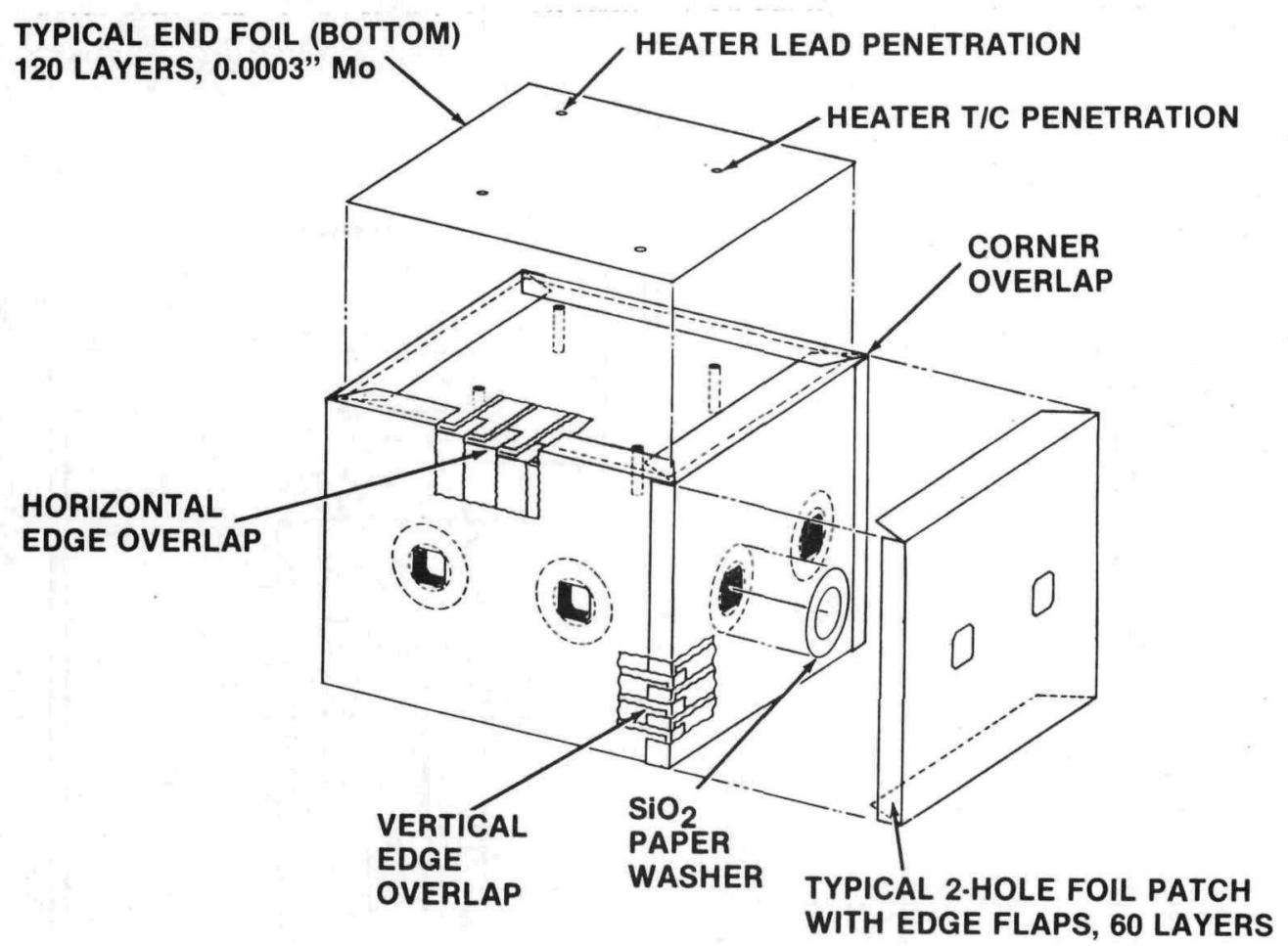


While the insulation package depicted in Figure 16 is very good thermally, it has one major disadvantage. The very snugness which keeps the heat in also tends to keep gases in, making it difficult to outgas. The initial test assemblies required more than a month to outgas without exceeding the prescribed pressure-versustemperature limits.

To reduce this delay, future test assemblies will have a conical port closed by a multifoil plug. As shown in Figure 17, the plug can be moved from outside the vacuum chamber by a magnetic actuator. Thus, the multifoil port will be open during outgassing, and closed after the assembly reaches its operating temperature.

Figure 17. Magnetically-Activated Outgassing Port

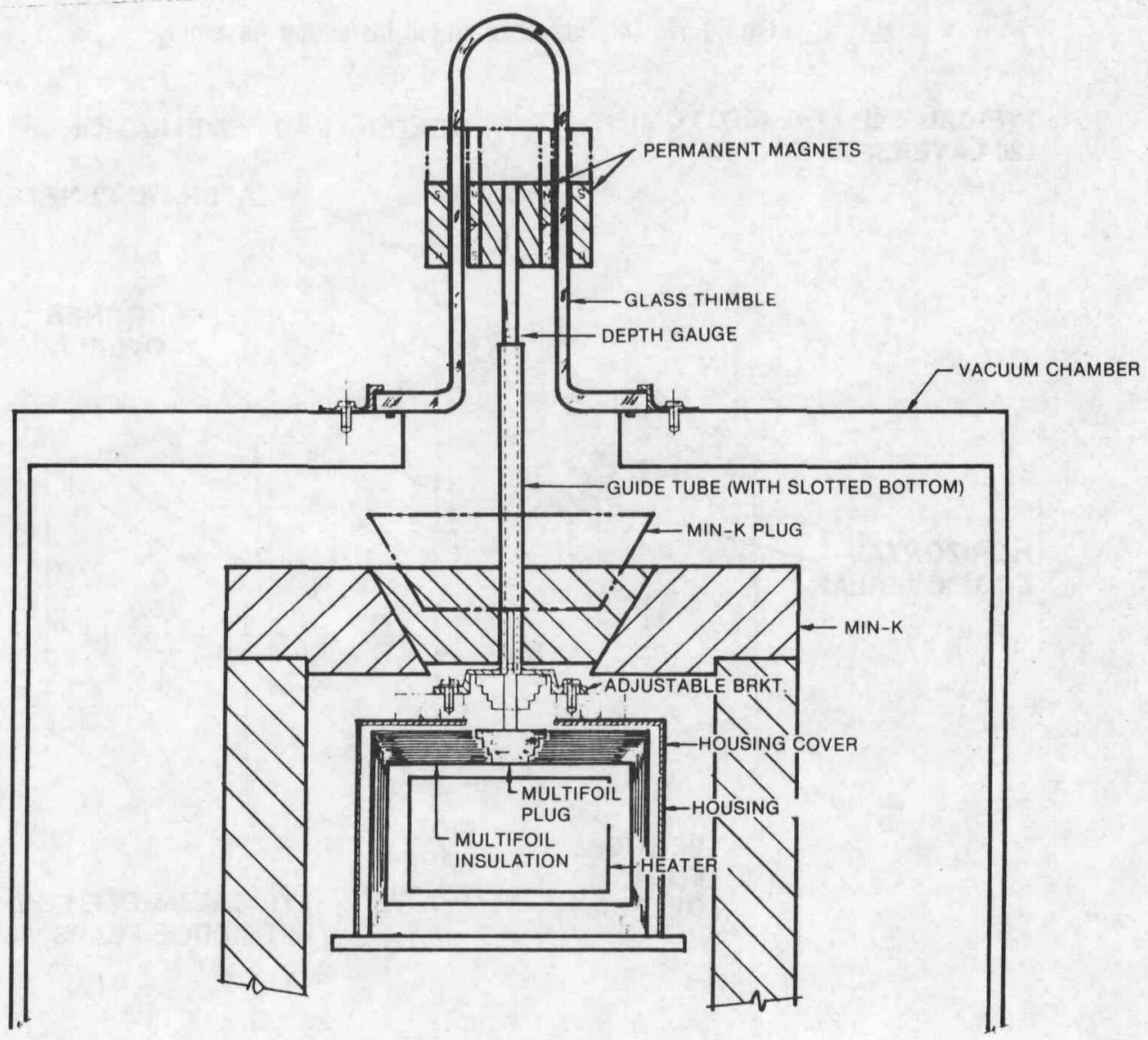




\section{Installation of Insulation and Multicouples}

The design and installation procedure for the multifoil insulation was devised and implemented by Thermo Electron. Since it consists of many individual patches with interleaved joints, and since perfect hole alignment is critical for later multicouple installation, the procedure is necessarily complicated. But it worked out very well, exactly as planned.

Figure 18. Collapsible Mandrel for Multifoil Lay-Up

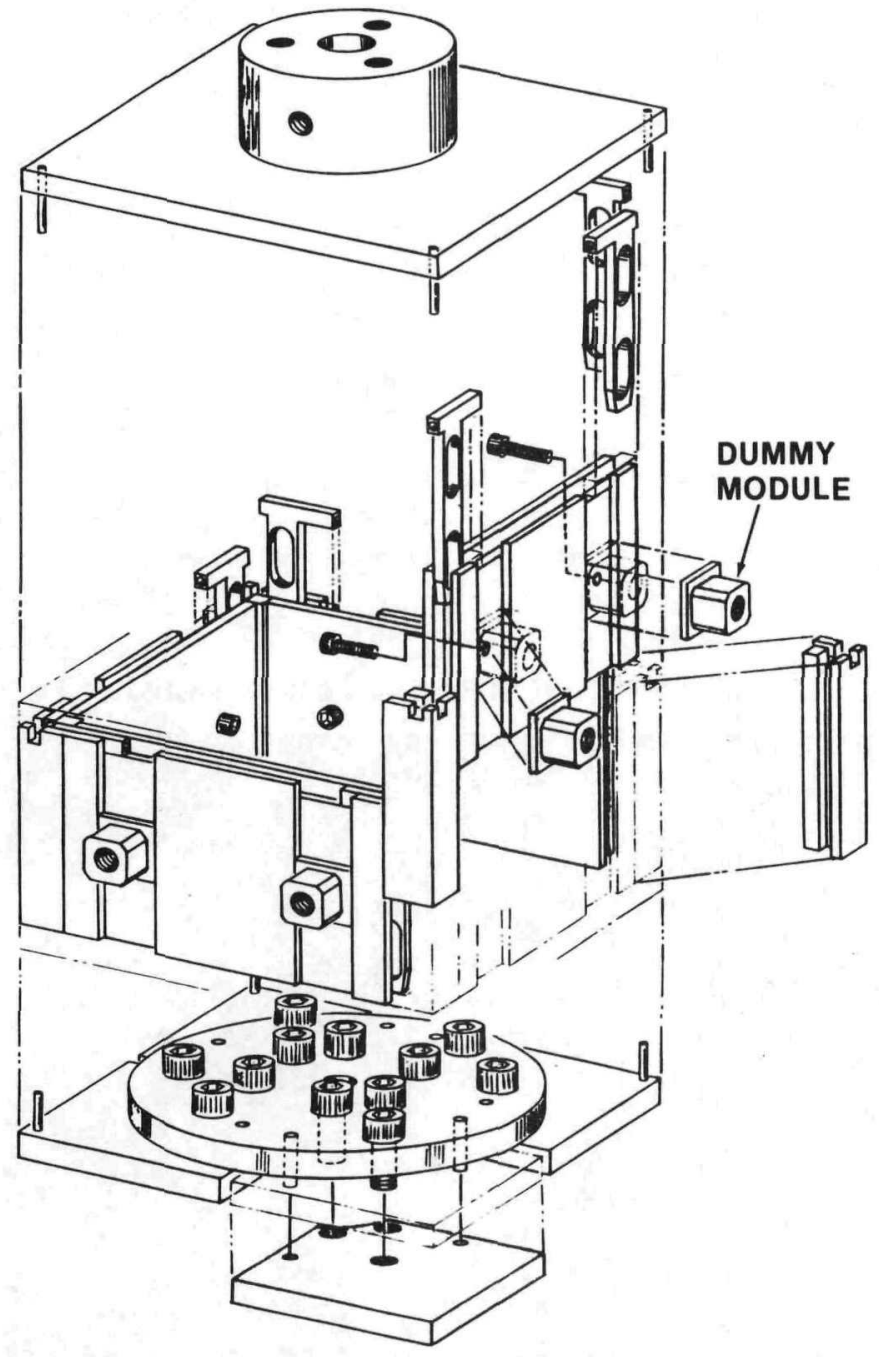

The procedure starts with a collapsible inner mandrel, as illustrated in exploded view in Figure 18. Bolted to its side walls are dummy modules at the eight multicouple locations. These dummy modules duplicate the dimensions of the multicouples, except for the heat collectors. 
Figure 19 depicts the assembled mandrel with dumnies, and Figure 20 shows it mounted on rotating base, ready for foil layup.

Figure 19. Assembled Mandrel with Dummy Modules

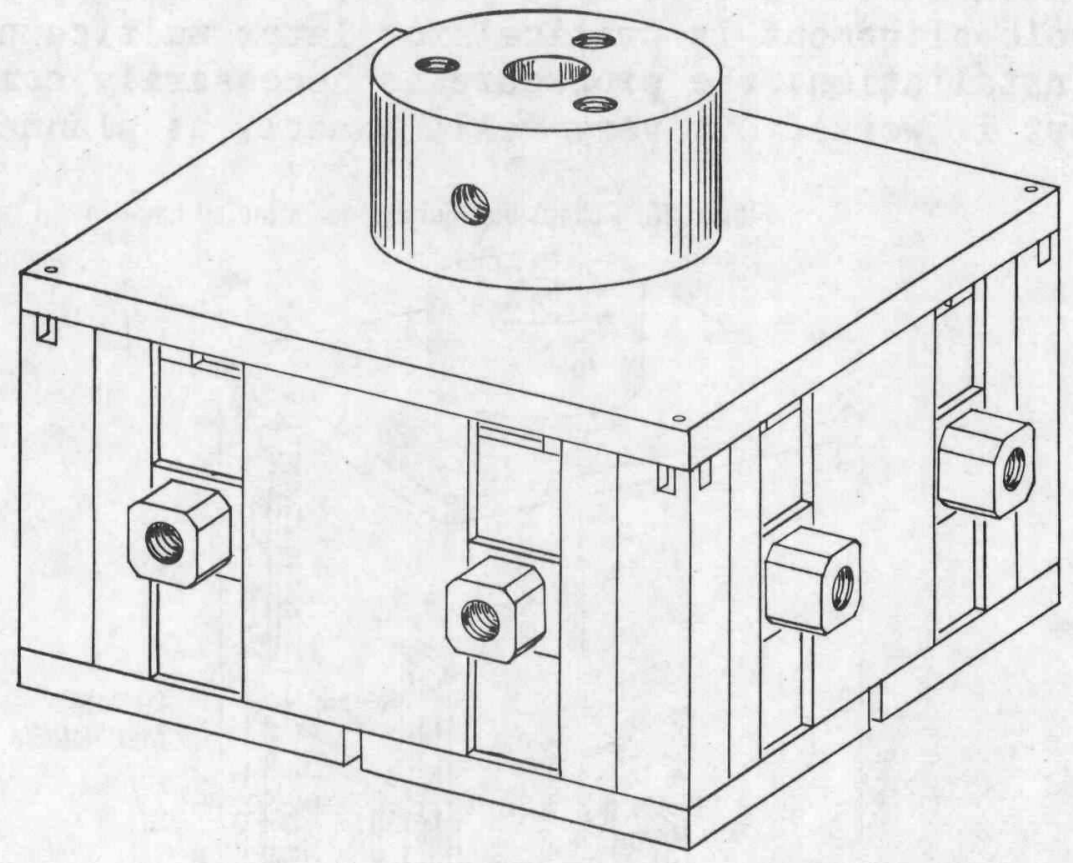

Figure 20. Mandrel on Rotating Stand for Interleaved Foil Lay-Up

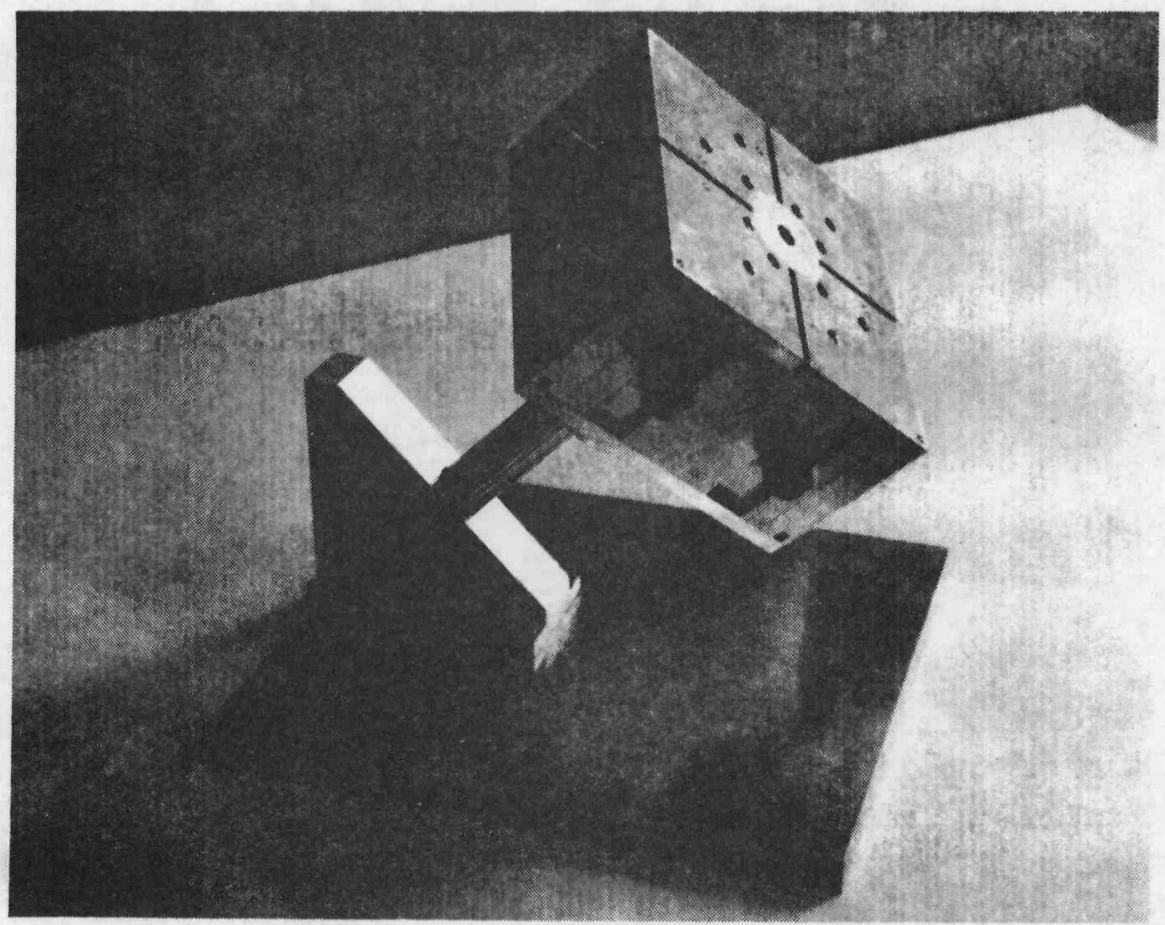


Figure 21 shows the mandrel after five of its faces have been covered with multifoil insulation, and Figure 22 shows the insulated mandrel installed in the housing side frame.

Figure 21. Foil Package Installed on Mandrel

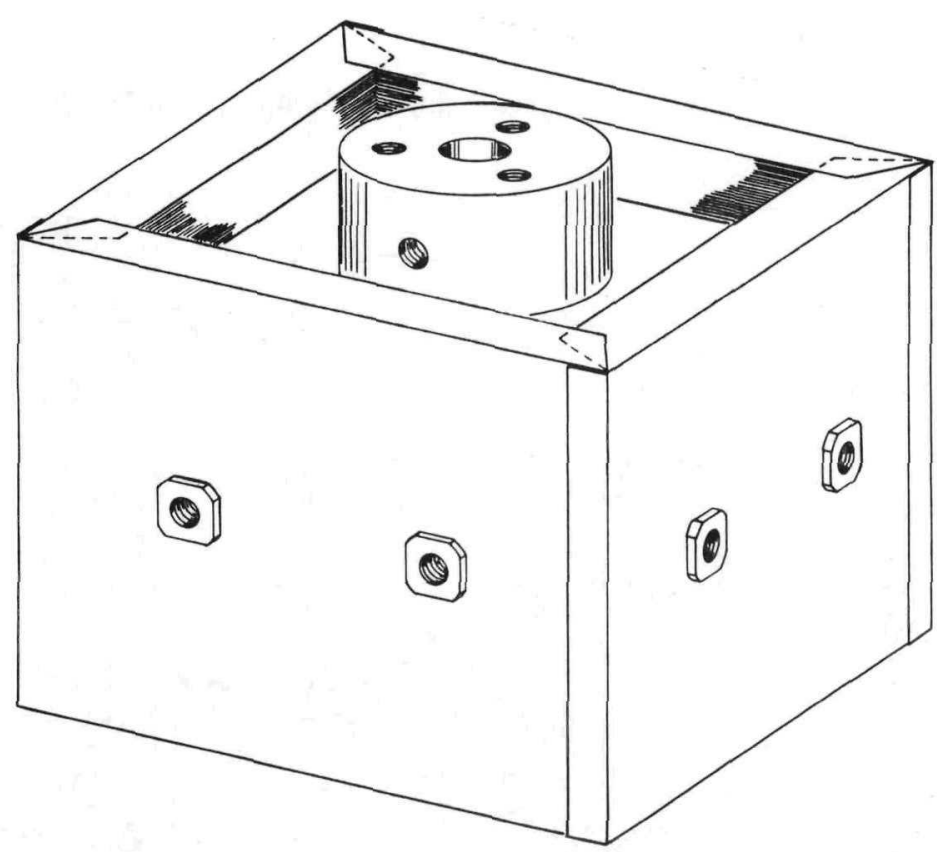

Figure 22. Foil Package Inserted into Housing

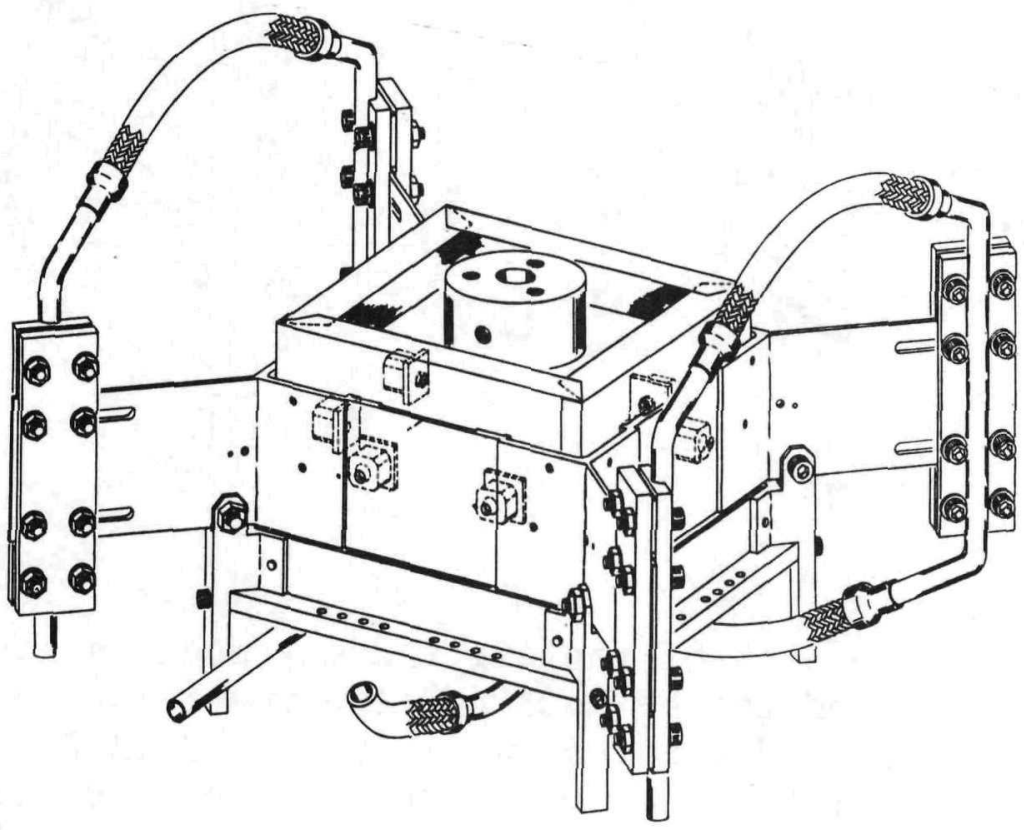


Figure 23 illustrates how each dummy module, one at a time, is unbolted from the inner mandrel and bolted from the outside to the corresponding multicouple mounting hole in the housing.

Figure 23. Transfer of Foils and Removal of Mandrel

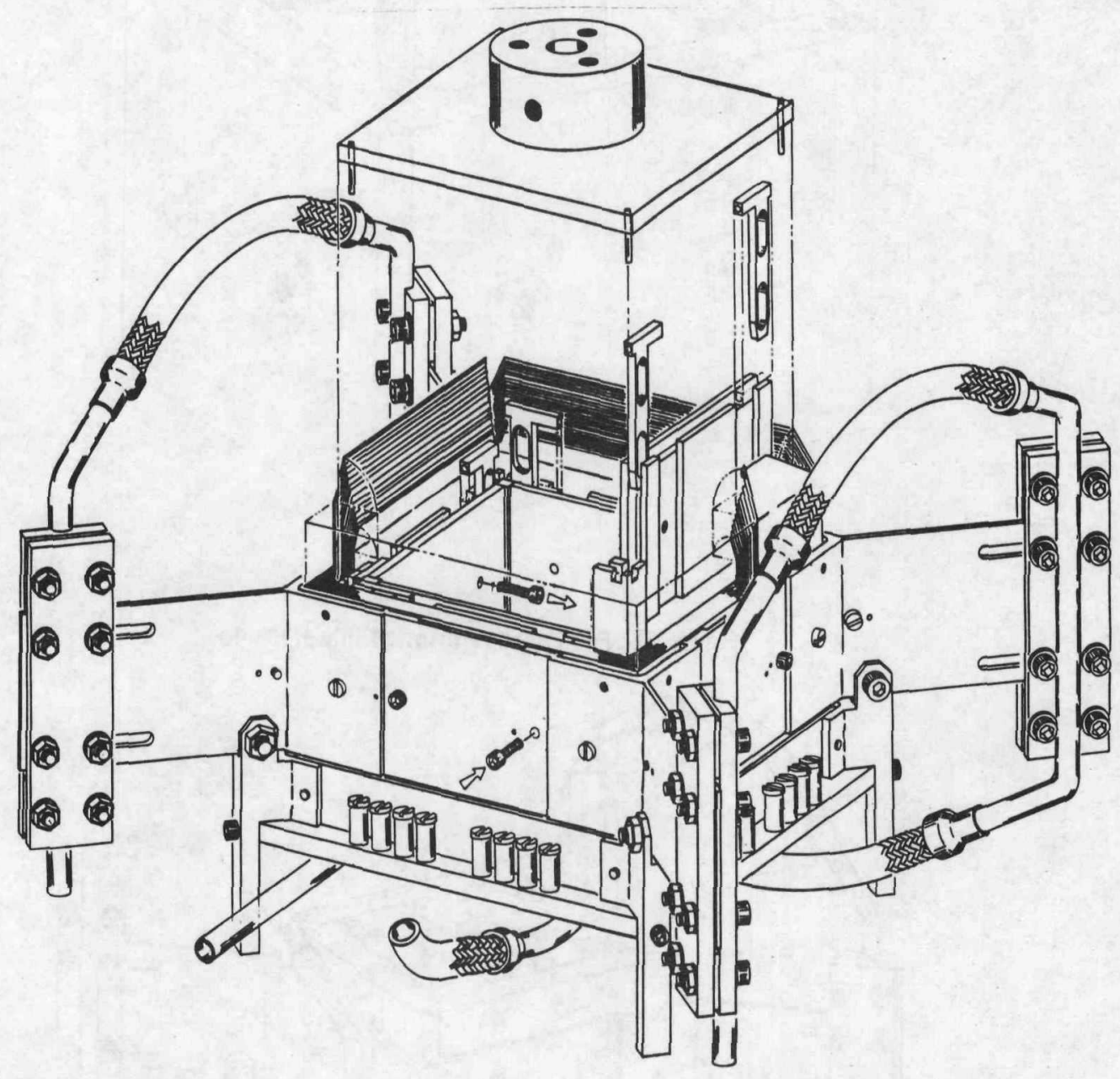

Once each of the eight dummy modules has been transferred to the housing, the inner mandrel is collapsed and removed, as illustrated in Figure 24 . At this stage, the foil holes in the insulated housing are maintained in proper alignment by the wall-mounted dummies, as shown in Figure 25. 
Figure 24. Assembly after Removal of Collapsible Mandrel

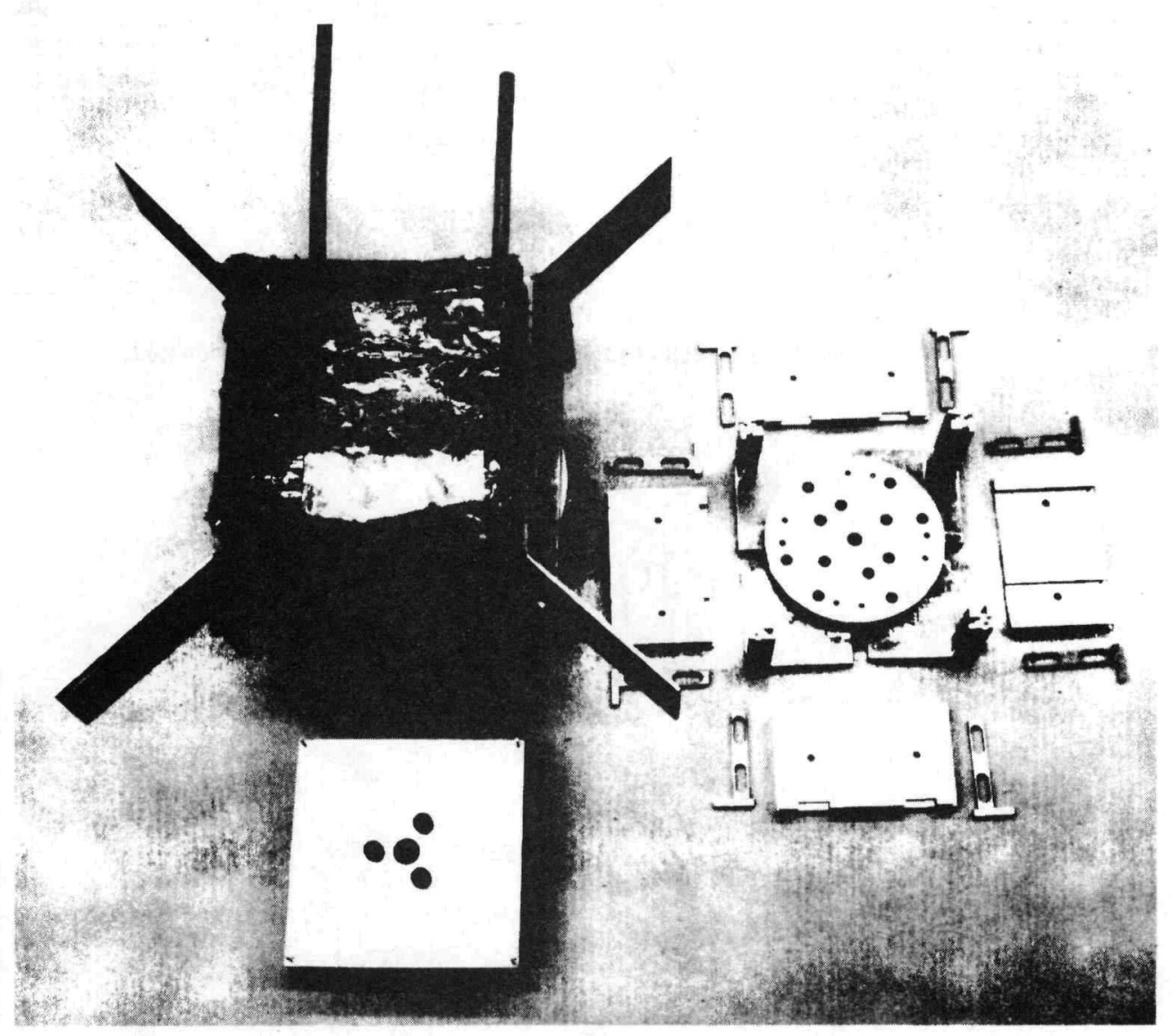

Figure 25. Insulated Housing with Foil Alignment Maintained by Dummy Modules

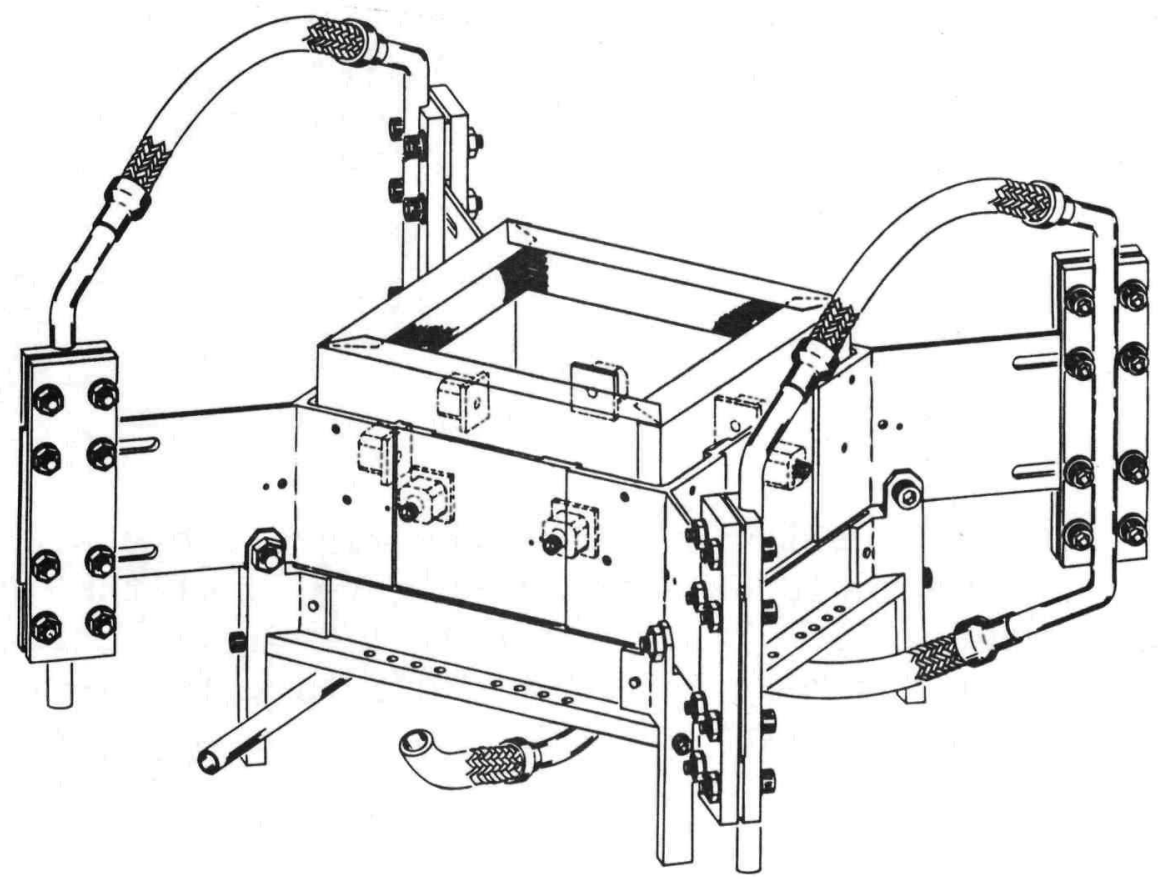


Finally, Figure 26 illustrates the replacement of the dummy modules by the multicouples. Since this replacement is done one at a time, proper alignment of the foils is always preserved.

Figure 26. Replacement of Dummy Modules by Multicouples

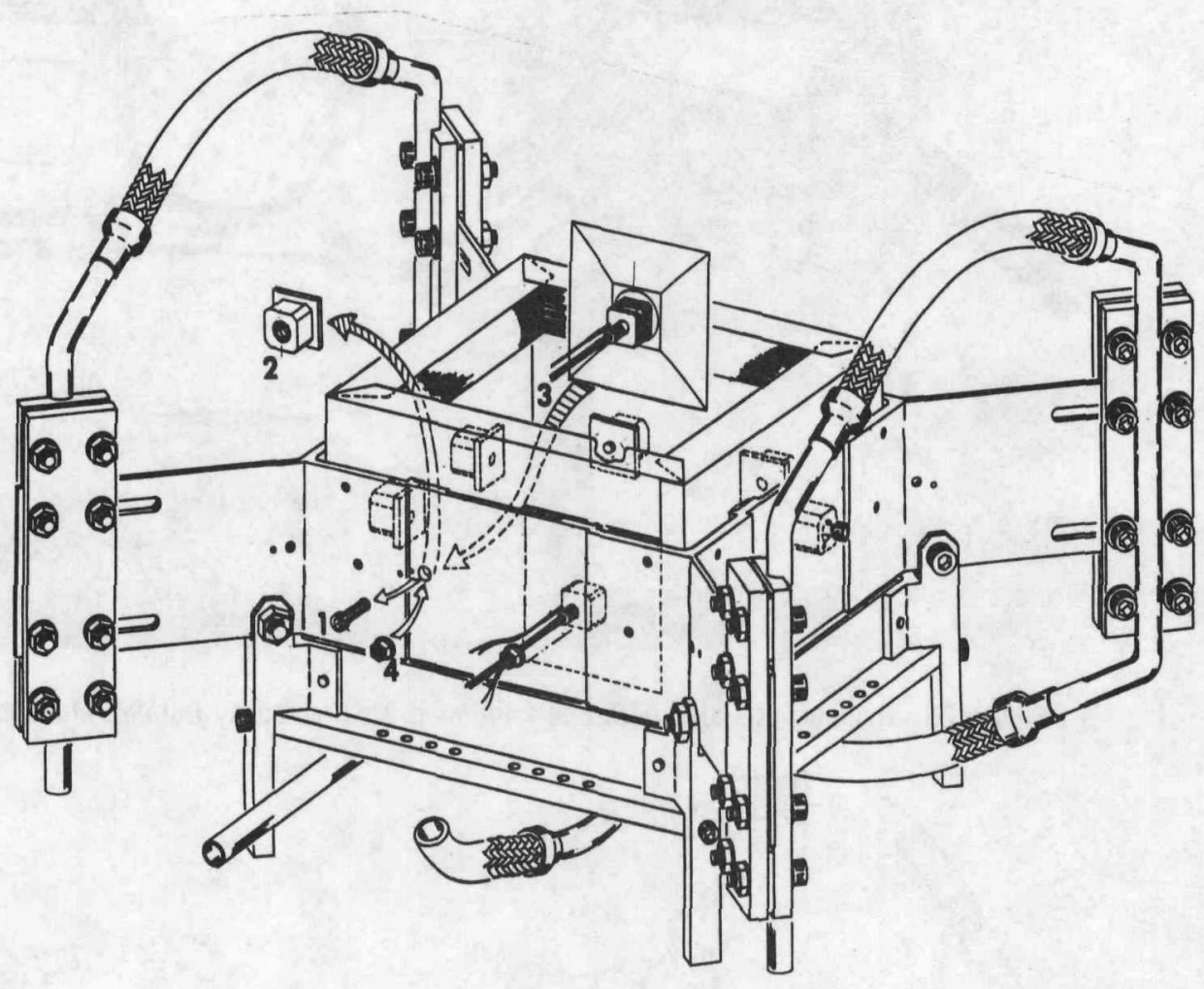

The installation steps described above are very similar to those depicted in Figure 30 of the author's 1981 paper [1]. Their successful implementation in the MITG test assembly enhances confidence in the feasibility of the MITG design. 
7. Installation of Heater and Closure of Test Assembly

The electrical heater assembly, built by Thermo Electron, is depicted in Figure 27. It consists of a continuous coiled HT molybdenum wire of $0.020^{\prime \prime}$ diameter, supported by four grooved alumina plates. The heater is capable of producing up to 400 watts at 120 volts.

Figure 27. Heater Assembly

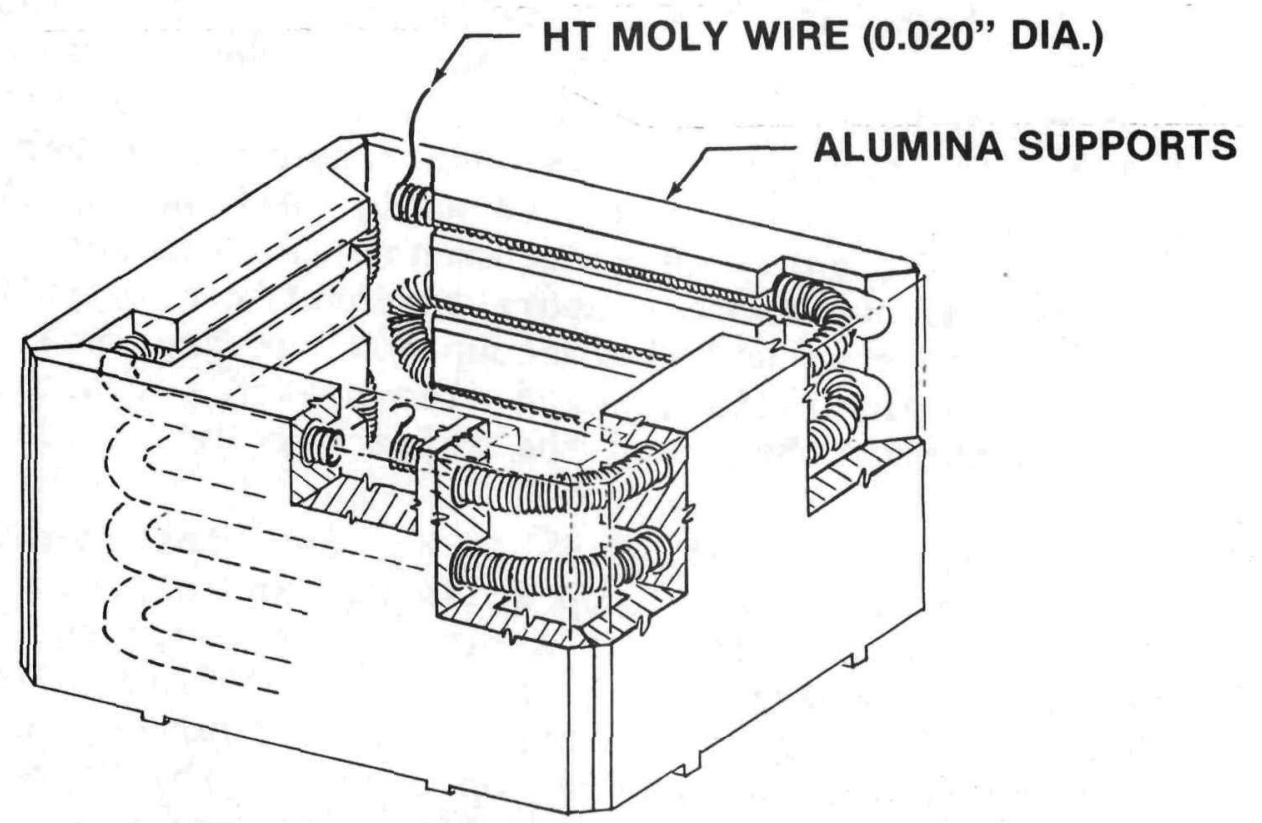

The four alumina plates form a box-like frame, which is inserted into the graphite heater box, shown in Figure 28, which has the same outer dimensions as the isotope heat source module.

Figure 28. Graphite Heater Box

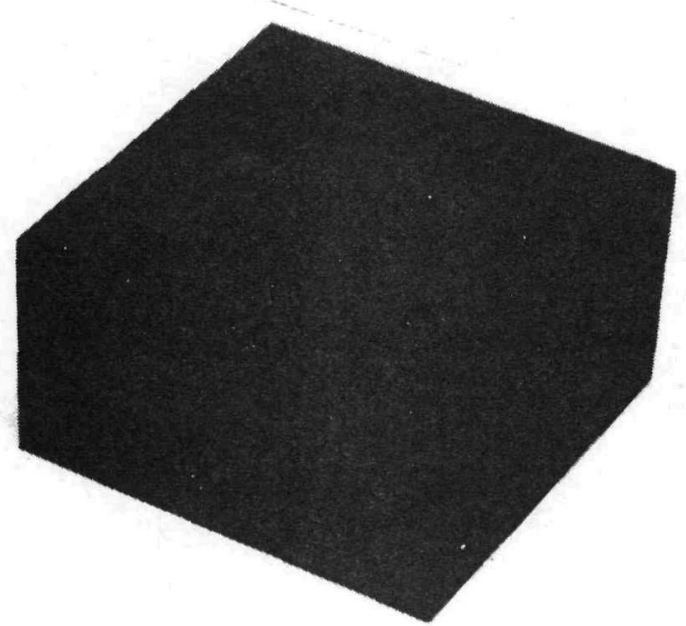


Figure 29 shows the heater assembly ready for insertion into the insulated converter. It also shows the top foils and top covers for completing the assembly.

As shown, the heater box has four small holes on the diagonals of its bottom face. Two holes, penetrating to the inside of the box, are for the heater leads. The other two holes, near opposite corners, are drilled into the graphite wall, half way up the heater box height. They accomodate two independent $\mathrm{W} / \mathrm{Nb}$ thermocouples to measure the heater temperature. The heater leads and thermocouple wires are enclosed in alumina insulation, which also prevents reactions between the graphite and the refractory metal wires.

After the heater assembly has been inserted into the converter, the 120 top foils are installed. This must be done two foils at a time, to produce the desired interleaving with the side foil flaps. As shown, the top foils are supported by four ceramic studs projecting above the top cover of the graphite heater box. These prevent deleterious reactions between the graphite and the molybdenum foils.

Finally, the top cover with its separate cooling loop is attached to the side frame. Again, for calorimetric reasons, there is no metal-to-metal contact. 
Figure 29. Insertion of Heater and Closure of Assembly

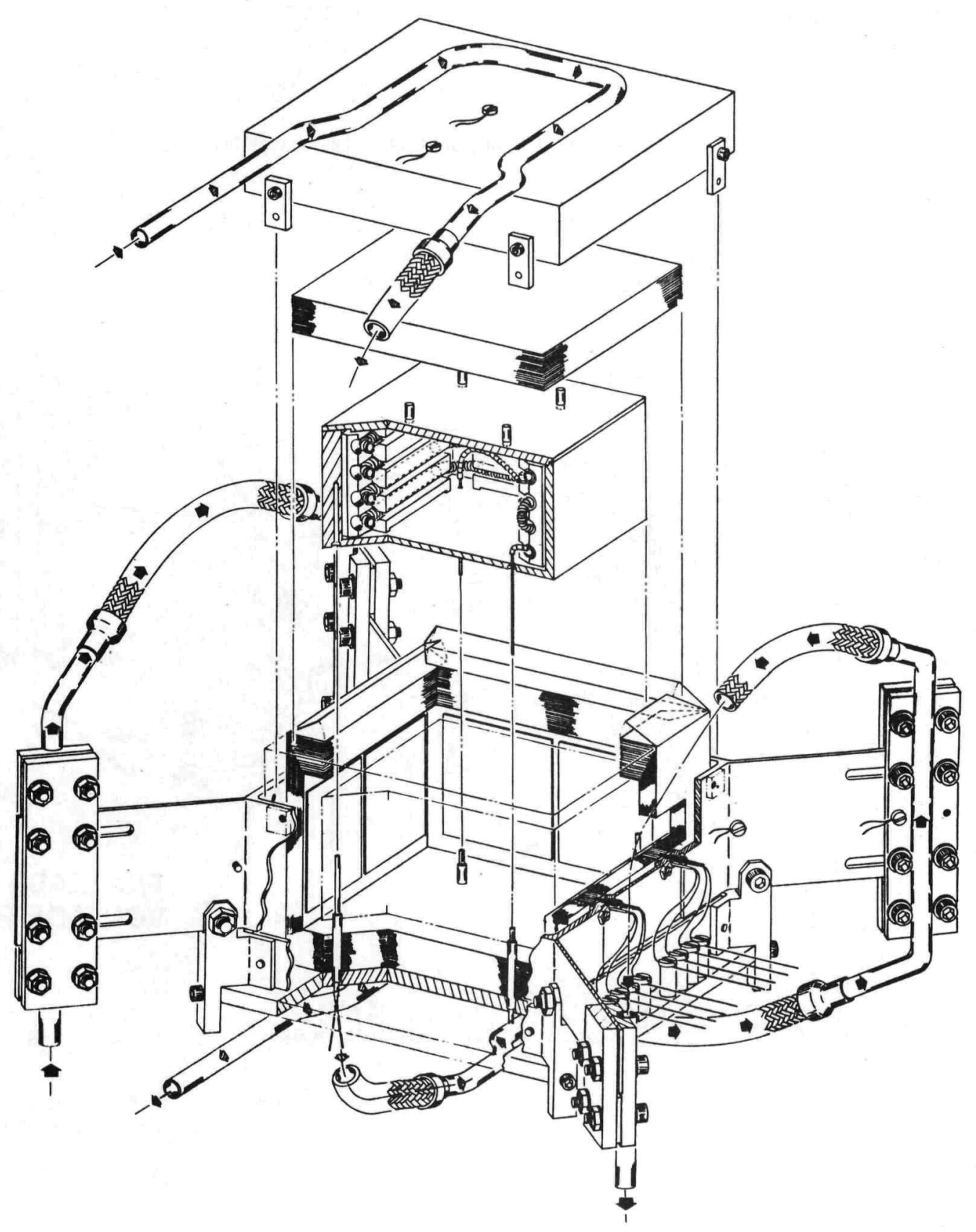


Figure 30 schematically shows the completed MITG test assembly, connected to its recirculating coolant loop. The cooling water comes from a controlledtemperature bath, which is used to adjust the coldjunction temperatures of the multicouples.

Figure 30. Completed MITG Test Assembly

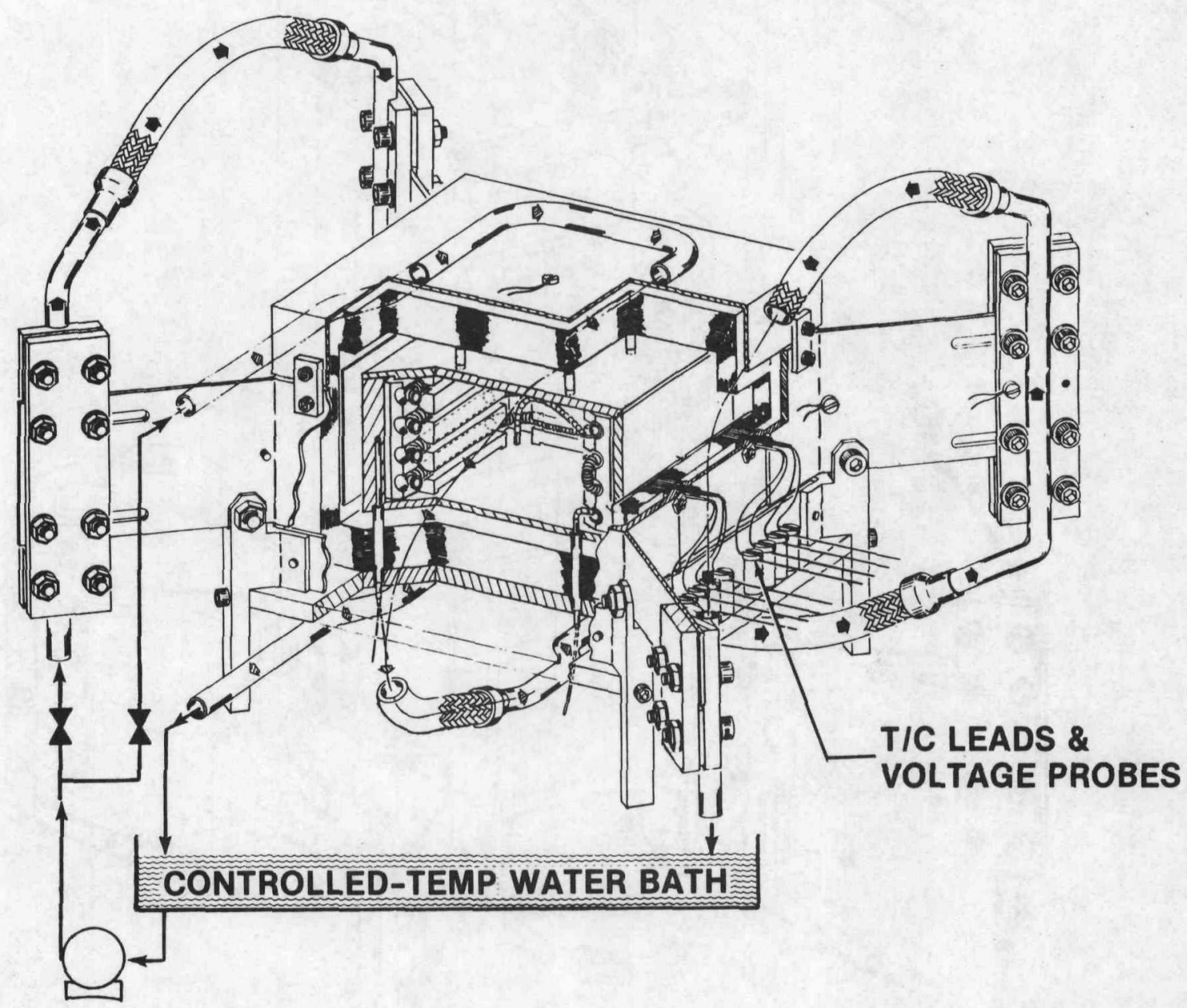




\section{Test Results}

Figure 31 shows the MITG test assembly being installed and instrumented in the vacuum test station, in preparation for the tests.

Figure 31. Test Assembly Installation in Vacuum Chamber

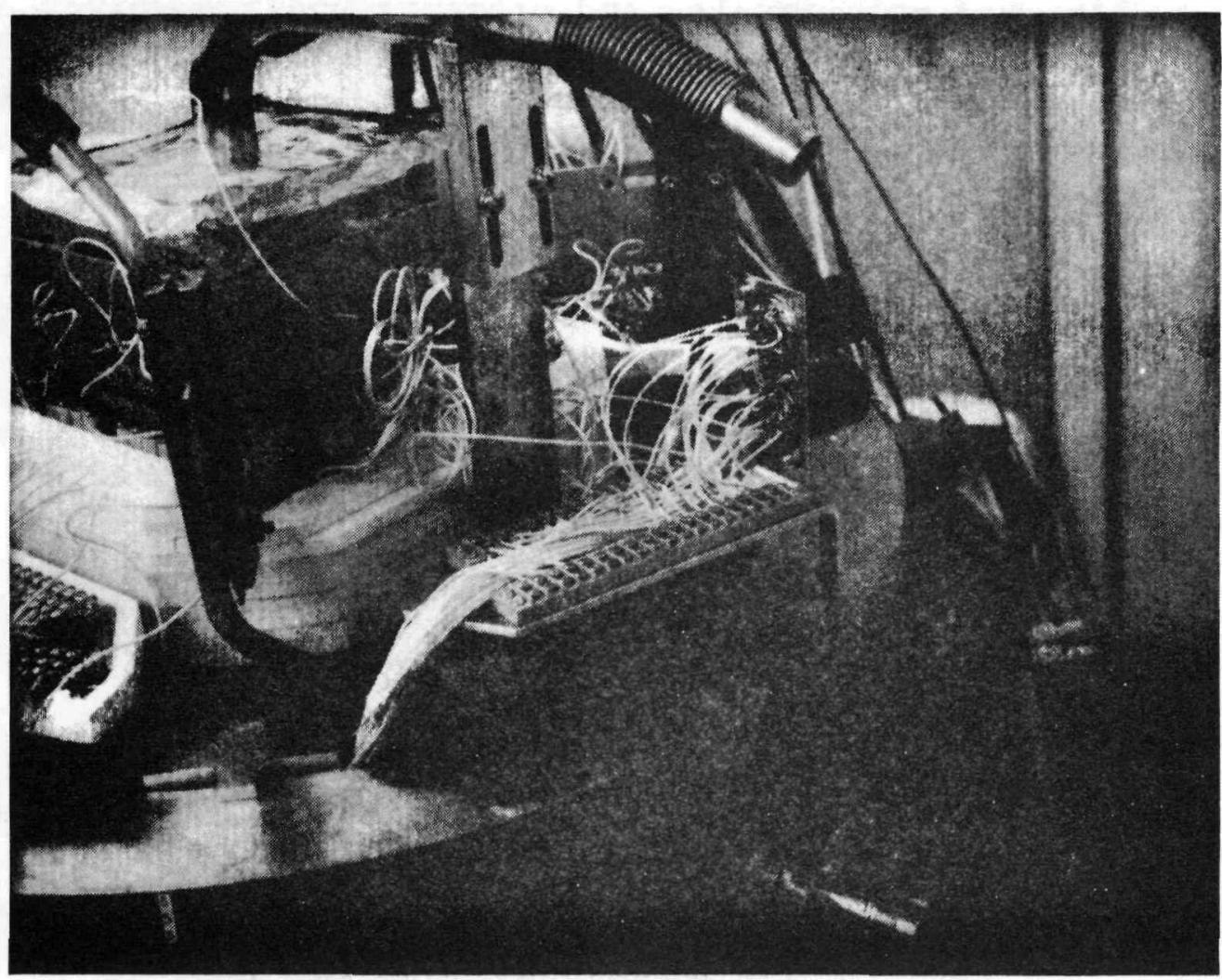


The test plan, methodology, instrumentation, computer control and data acquisition systems, and the initial test results are described in the next paper in these proceedings [3]. The following paper [4] presents the results of the post-test examination of the test hardware, including a very detailed thermal stress analysis, which succeeded in identifying the principal causes of the observed thermal-stress problems and suggesting solutions capable of eliminating those problems. The last paper in this series [5] describes a number of additional design modifications for an improved multicouple, and presents updated weight and performance predictions for MITG flight generators incorporating those design modifications as well as the latest thermoelectric property measurements.

\section{Acknowledgment}

The author takes pleasure in acknowledging that the work described here reflects the contributions of many individuals, including (alphabetically): M. Eck, T. Kline, and M. Mukunda of Fairchild; V. Raag and L. Waddingham of Syncal; and F. Huffman, S. Matsuda, and G. Miskolczy of Thermo Electron.

\section{References: (Part B):}

\section{Part:}

A. [1] A. Schock, "Modular Isotopic Thermoelectric Generator", Proceedings of the 1981 IECEC, Volume 1, Pg. 327.

[2] A. Schock, "Design Evolution of the General-Purpose Heat Source", Proceedings of the 1980 IECEC, Volume 2, Pg. 1032.

C. [3] M. Eck, M. Mukunda, "MITG Test Procedure and Results", Proceedings of the 1983 IECEC, next paper.

D. [4] A. Schock, "MITG Post-Test Analysis and Design Improvements", Proceedings of the 1983 IECEC, following paper.

E. [5] A. Schock, "Revised MITG Design, Fabrication Procedure, and Performance Predictions", Proceedings of the 1983 IECEC, subsequent paper. 


\title{
Part C:
}

\section{MITG Test Procedure and Results}

\author{
Marshall Eck, Meera Mukunda \\ Fairchild Space Company \\ Germantown, Maryland 20874
}

(Presented at the 1983 Intersociety Energy Conversion Engineering Conference)

\begin{abstract}
Elements and modules for Radioisotope Thermoelectric Generators have been performance tested since the inception of the RTG program. These test articles seldom resembled flight hardware and often lacked adequate diagnostic instrumentation. Because of this, performance problems were not identified in the early stage of program development. The lack of test data in an unexpected area often hampered the development of a problem solution.

A procedure for conducting the MITG Test was developed in an effort to obtain data in a systematic, unambiguous manner. This procedure required the development of extensive data acquisition software and test automation. The development of a facility to implement the test procedure, the facility hardware and software requirements, and the results of the MITG testing are the subject of this paper.
\end{abstract}




\section{INTRODUCTION AND SUMMARY}

Fairchild Space Co. personnel under contract to the U.S. Department of Energy (DOE), conducted performance tests on a total of 20 multicouples for the Modular Isotope Thermoelectric Generator (MITG) described in Reference 1. These multicouples, the details of which are presented in Reference 2, were fabricated by Syncal Corp., also under DOE contract. The multicouples measured 0.340 inches square $\times 0.300$ inches long. Each multicouple was fitted with a heat collector and a cold mounting stud. They were fabricated from a $6 \times 6$ array of $\mathrm{n}$ and $p$ silicon germanium ( $\mathrm{SiGe}$ ) or gallium phosphide (GaP) modified SiGe thermoelectric (T/E) legs. Eight multicouples were mounted in a prototypical MITG housing slice and tested as a unit. The rationale for testing at this level of assembly and a detailed description of the test article was presented in a companion paper (Ref. 2). A cutaway drawing of the MITG prototypical module tested is shown in Figure 1.

The test assembly was heated electrically but the electrical heater was enclosed in a graphite box having the same outer dimensions as the General Purpose Heat Source (GPHS) re-entry module. The test assembly contained eight MITG multicouples and prototypic sections of the generator housing, and the proposed foil insulation. The top and bottom of the heater box were particularly well insulated to minimize non-prototypic heat losses in the housing covers. The housing covers were mounted on insulators to the housing side wall. This minimized heat conduction between the prototypical and non-prototypical portions of the test assembly. As in the MITG, heat from the prototypic housing wall flows to the corner fins. Heat was removed from the fins by circulating water through tubes which were brazed to clamp-on copper saddles. Heat was removed from the top and bottom housing covers by separate instrumented circulating water circuits. 


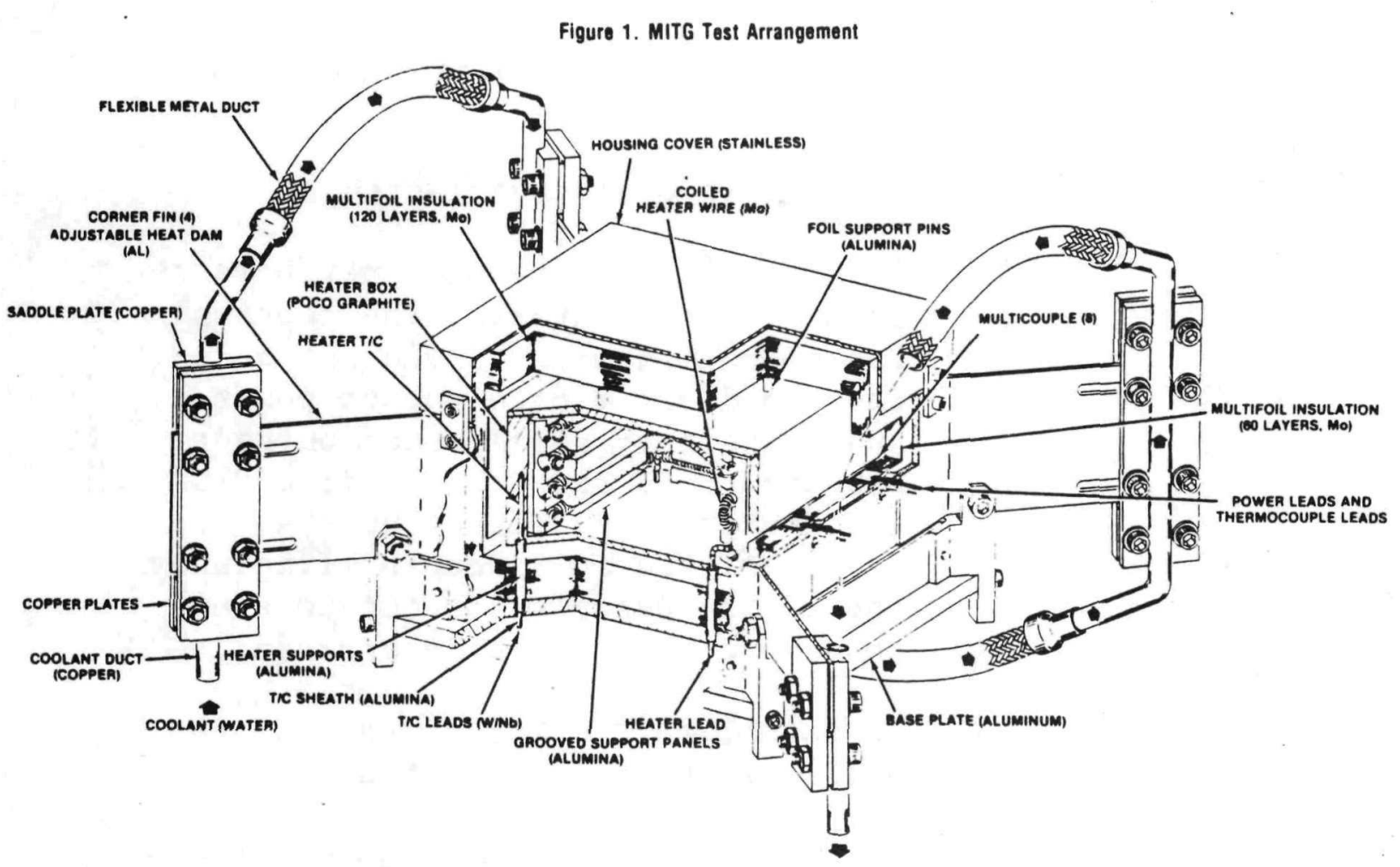

Four test assemblies (modules) were fabricated. Two were used in the subject test program and two were reserved for future testing. One module contained the standard SiGe material and one contained the GaP modified material. Each was tested at the reference hot junction temperature of $1000^{\circ} \mathrm{C}$. Cold junction design temperature was $300^{\circ} \mathrm{C}$. However, in practice, there was a large variation in the cold junction test temperature.

Extreme care was taken in the design of the test assembly and the test facility to maximize the accuracy of data taken for efficiency and heat balance determinations. It was essential that good calorimetry be performed, since the establishment of multicouple efficiency and a determination of the differences in performance between the stand.ard SiGe and the GaP modified SiGe $T / E$ material were the primary objectives of this test. 
The four major test objectives were:

1. To establish multicouple performance

in a systems context. The testing was conducted with sufficient accuracy to discriminate output power and efficiency differences between standard SiGe and GaP modified SiGe in identical systems. The absolute values of the multicouple efficiency and of the degradation due to shunt loss were also to be determined.

2. To establish the performance of the module's thermal insulation.

3. To estimate potential module lifetime by careful measurement of the degradation of the individual multicouple's power output. In this manner, it was possible to determine whether a single multicouple was the cause of test assembly degradation or whether the individual multicouples were degrading approximately equally. This also allowed segregation of the degradation effects due to shunt resistance and to degradation mechanisms essentially associated with the $T / E$ materials.

4. To make parametric variations in the $T / E$ module voltage and cold junction temperature of $\pm 100^{\circ} \mathrm{C}$ around the design value of $300^{\circ} \mathrm{C}$ while holding the hot junction constant at its design value of $1000^{\circ} \mathrm{C}$. 
Meeting these ambitious test objectives required design and development of a highly reliable test facility. This established the need to acquire reliable and very clean vacuum bell jars, and to design a highly adaptive control and data acquisition system. The design and operation of this test facility is the principal subject of this paper.

The general arrangement of the MITG test facility is shown in Figure 2. The facility consisted of four turbomolecular/Vac-Ion pumped bell jars and two CVC diffusion pumped bell jars. Only two turbomolecular/ Vac-Ion stations were used for the subject tests.

Figure 2. Perspective Drawing of MITG Test Facility

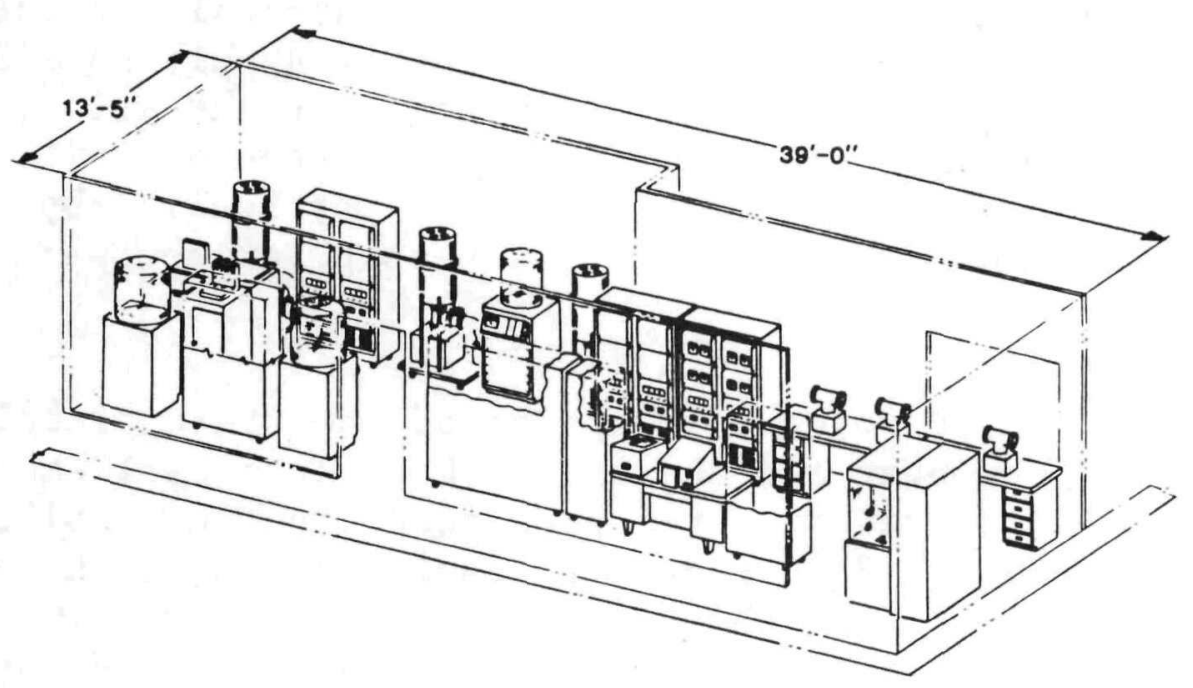


The tests were conducted automatically using diagnostic, control, and data acquisition software specifically written by Fairchild personnel for this purpose. The heart of the control system was a Hewlett Packard (HP) $21 \mathrm{MXE}$ computer with $576 \mathrm{~K}$ bytes of fast core and 2.3 megabyte hard disc. The computer listened to the system through an HP 3965 scanner and addressed the system through an HP 6940 multiprogrammer. HP 3455 and HP 3456 DVMS were, used to digitize the analogue input. Separate control data acquisition and safety functions were provided to minimize potential equipment failure problems. Battery backup for the computer and diesel backup for the test facility were provided. Software was written to provide an automatic restart capability. Post-processing software was written to provide automatic data reduction and plotting.

Figure 3 and 4 are typical of the data reduction performed during the test program. The predicted Seebeck coefficient (alpha) of the single multicouple is seen to track the theoretical value rather closely until a catastrophic event occurred. The theoretical and measured alpha curves were obtained by post-processing, using the measured multicouple hot and cold junction temperatures and the open circuit voltage. The internal resistance was obtained using a stiff power supply as a load, varying its voltage, measuring current flow, and calculating the multicouple resistance. All these functions were performed automatically by the control and data acquisition computer software.

A detailed description of the test facility, the computer and the software which established the test procedure are presented in the following chapters. Selected test results are also shown. A description of the steps taken to overcome the multicouple mechanical problems uncovered by the subject testing is presented in a separate paper (Ref. 2). 
Figure 3. Seebeck Coefficient

STATION 1 MULTICOUPLE 1

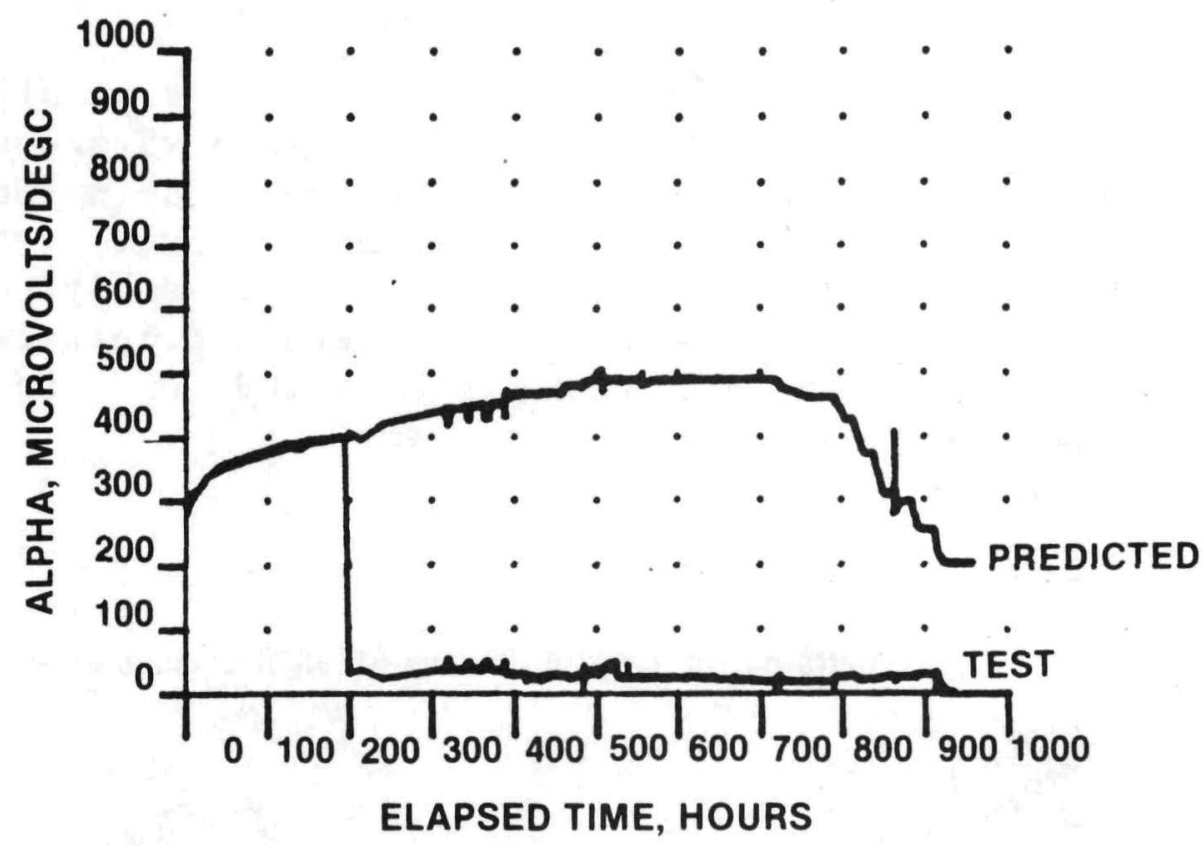

Figure 4. Seebeck Coefficient

STATION 1 MULTICOUPLE 2

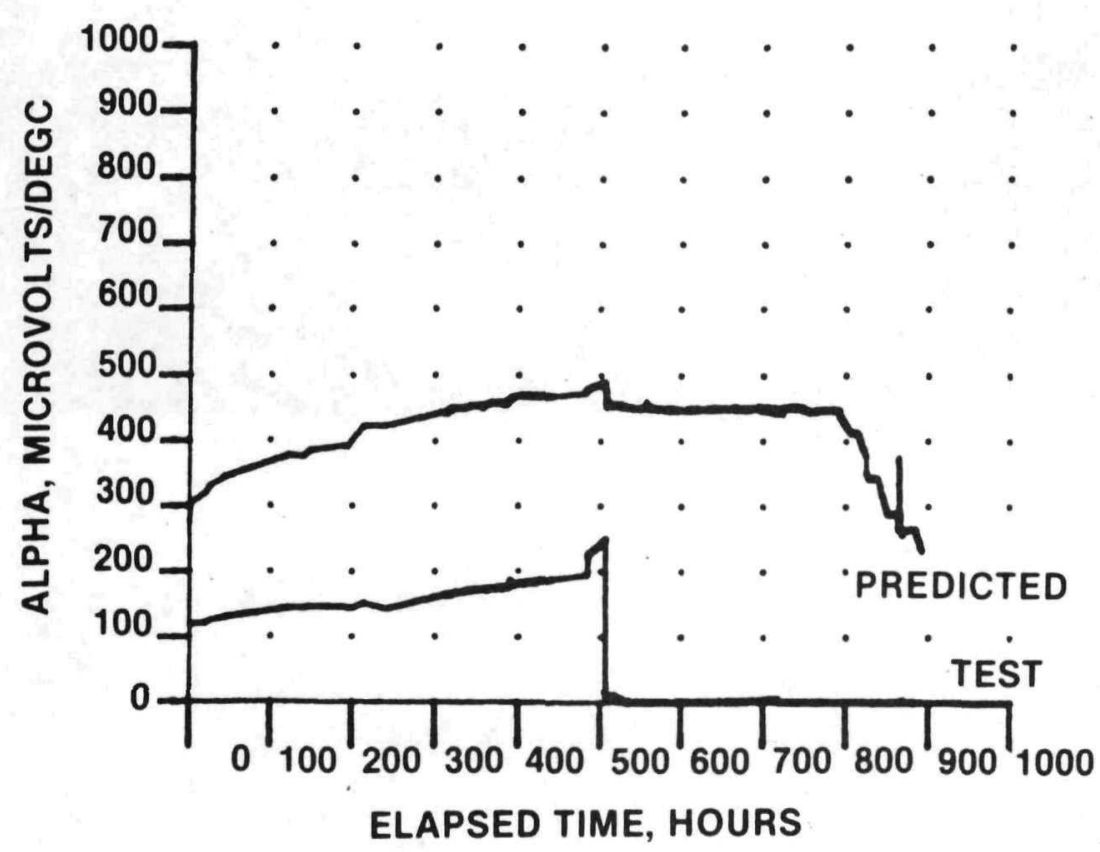


II. DISCUSSION

A brief description of the test assembly was presented in the Summary. Figure 5 shows this article partially assembled and mounted in one of the turbomolecular/Vac-Ion pumped vacuum stations. A detailed description of the test assembly and the rationale for its design is presented in reference 2. The paper at hand is focused on the test operations of the MITG program.

Figure 5. Top View of Partially Assembled Module

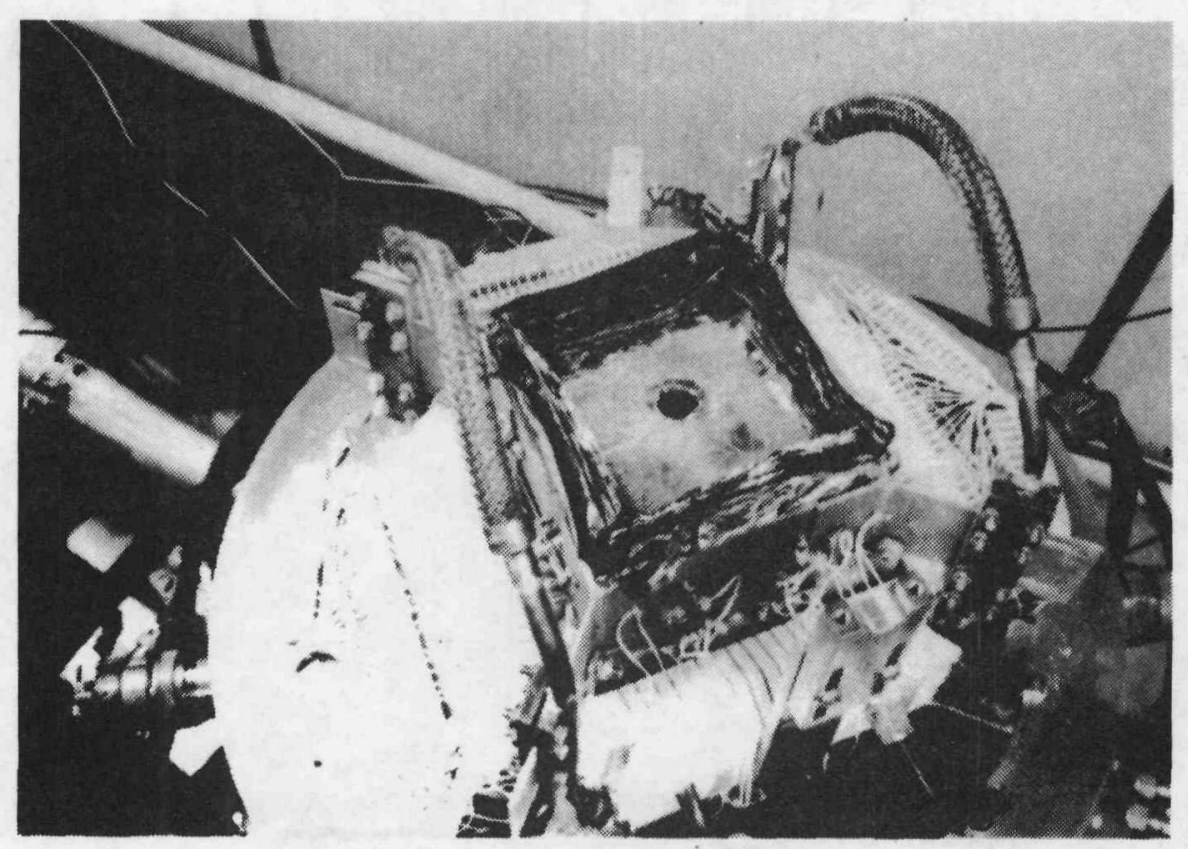


The MITG test facility was designed and built in less than six months. Although Fairchild personnel were authorized to begin planning the facility in December 1981, authorization to begin construction was not received until February 1982. Multicouple testing was begun in May 1982. The use of a substantial amount of Government Furnished Equipment (GFE) greatly reduced procurement lead time. The computer, data acquisition system, vacuum stations and a number of accessories were obtained as surplus from various DOE facilities. This equipment was received at the Sherman Fairchild Technology Center in Germantown, Maryland, in January 1982. Substantial modifications to most of the GFE were accomplished after their receipt.

The MITG area was selected, partitioned and provided with utilities in the period 15 February through 15 April, 1982. This effort was complicated by the need to install a separate 150 amp 220 VAC electric service from the diesel backed emergency bus. The test equipment installation was completed in late May 1982 just prior to the receipt of the test assembly components from Syncal. Figure 6 is a photograph of the MITG test area and the computer arrangement.

Figure 6. General MITG Test Area

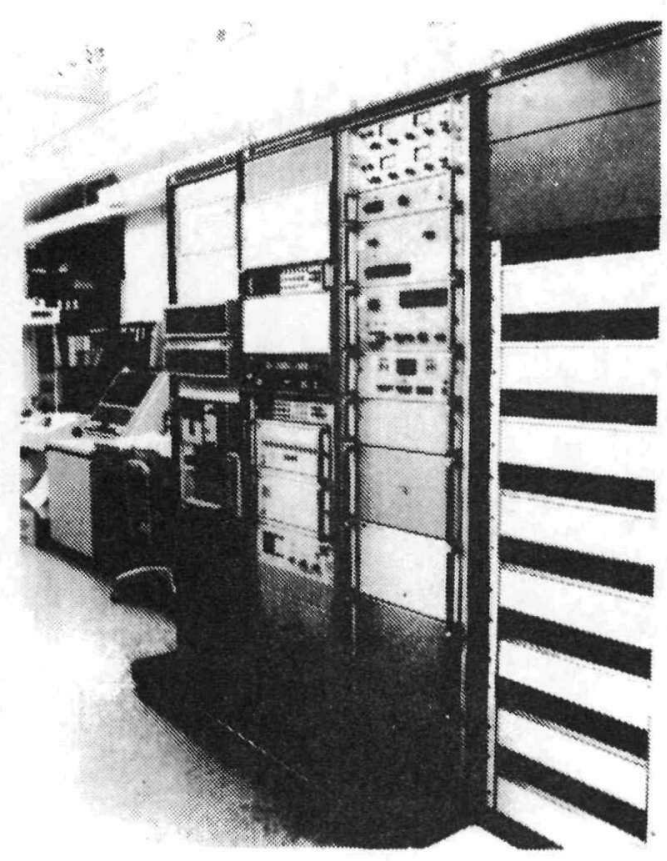




\section{A. Vacuum Stations}

Six vacuum stations are available in the MITG test facility. Two turbomolecular/Vac-Ion pumped 18 inch diameter by 30 inch high stainless steel bel1 jars were selected for testing the MITG modules. A Welch mechanical pump was used to rough the bel1 jars and as a foreline pump for the turbomolecular pumps.

Each chamber was fitted with five 26 pin electrical feedthroughs to accommodate the need for electrical and instrumentation penetrations. Heater power and module output power were carried by a single 7 pin feed through. Separate voltage taps from the heater were also carried through the 7 pin feedthrough. These taps were necessary to remove the uncertainty of power wiring voltage drop from the calculation of input power. A photograph of a typical turbomolecular/Vac-Ion pumped vacuum station is shown in Figure 7 .

Figure 7. Typical Turbomolecular/Vac-Ion Pumped Vacuum Station

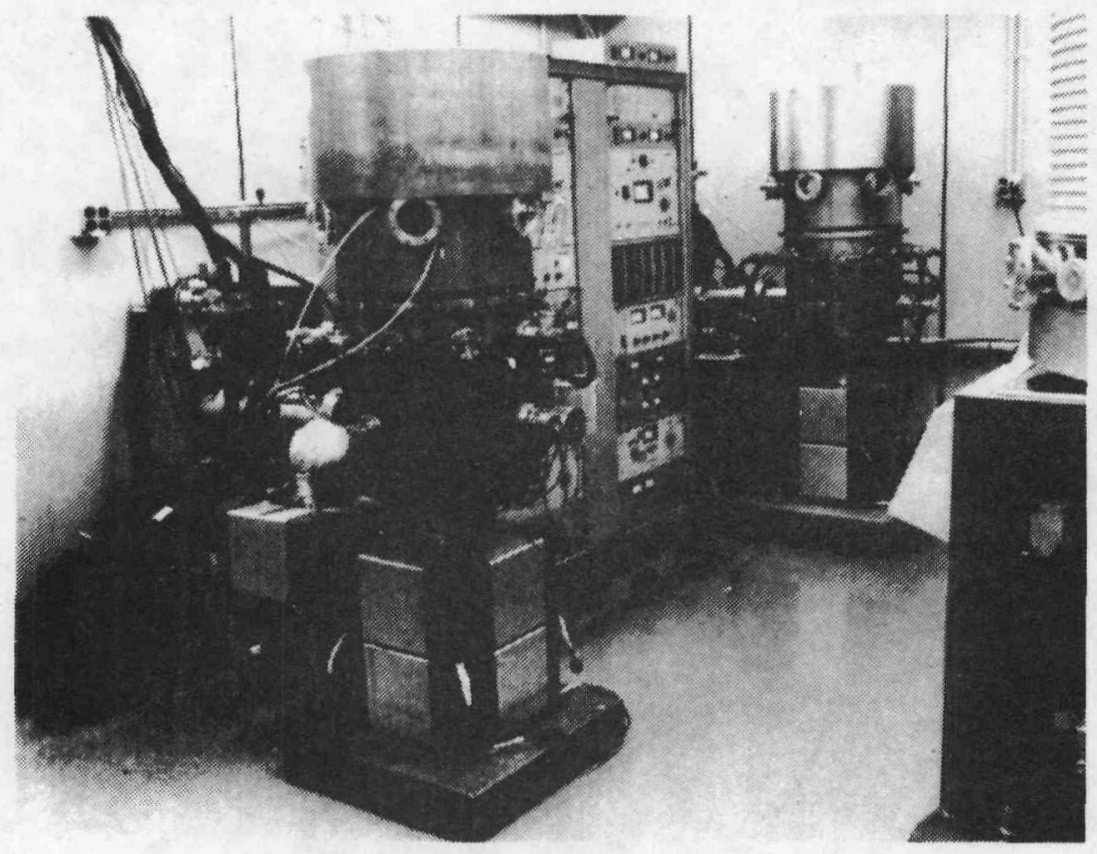


Testing in vacuum at temperatures of $1000^{\circ} \mathrm{C}$ and at pressures less than $10^{-6}$ Torr requires high pumping capacity. None of the components assembled into the test article were planned to be outgassed at their design temperatures before being installed in the test vacuum station. Because of this, a decision was made to modify the GFE chambers to add pumping capacity and to increase conductance for the existing pumps.

The chambers as received were mounted on the inlet flange of an ULTEK (now Perkin-Elmer) $550 \mathrm{l} / \mathrm{s}$ Vac-Ion pump. The chambers were roughed by a 450 $1 / \mathrm{s}$ Leibold- Hereus turbomolecular pump using a Welch pump to a suitable foreline pressure. As received, the roughing port was only $2-1 / 2$ inches in diameter. This port was blanked and a six inch port, tee, and elbow were added to accommodate an additional $220 \mathrm{l} / \mathrm{s}$ Perkin-Elmer Vac-Ion pump and to provide adequate conductance to the turbomolecular pump. A six inch gate valve was used to isolate the turbomolecular pump from the chamber. This arrangement allowed unattended operation with very little chance of test article damage due to air in leakage in the event of loss of facility electric power.

The vacuum stations performed very we11. Clean, dry and empty, they blanked off at $5 \times 10^{-8}$ Torr. After 1000 hours of testing at heater temperatures as high as $1100^{\circ} \mathrm{C}$, the chamber pressure was steady at $8 \times 10^{-7}$ Torr with the turbomolecular pump secured and both Vac-Ion pumps operating. The turbomolecular pump was very useful in outgassing the test assembly. The Vac-Ion pumps would not start above $5 \times 10^{-6}$ Torr. As a result, signficiant system processing was performed using just the turbomolecular pumps. Once the pressure fell below $2 \times 10^{-6}$ Torr, Vac-Ion pump operation was very stable and they provided adequate pumping capacity. 


\section{B. Computer}

All MITG testing, as discussed earlier, was under computer control. The hardware used for this function was manufactured by Hewlett-Packard, and except for a few new purchases, it was obtained as Government Furnished Equipment (GFE). A brief description of the computing hardware is given below.

1. Main Frame HP 21 MXE with $576 \mathrm{~K}$ bytes of fast core and a 2.3 megabyte hard disc.

2. Multiprogrammer HP 6940B.

3. Magnetic Tape Unit HP 7970E.

4. Line Printer HP 2631B.

The functional design of the MITG test assembly is shown in Figure 8 .

Figure 8. MITG Test Assembly Functional Design

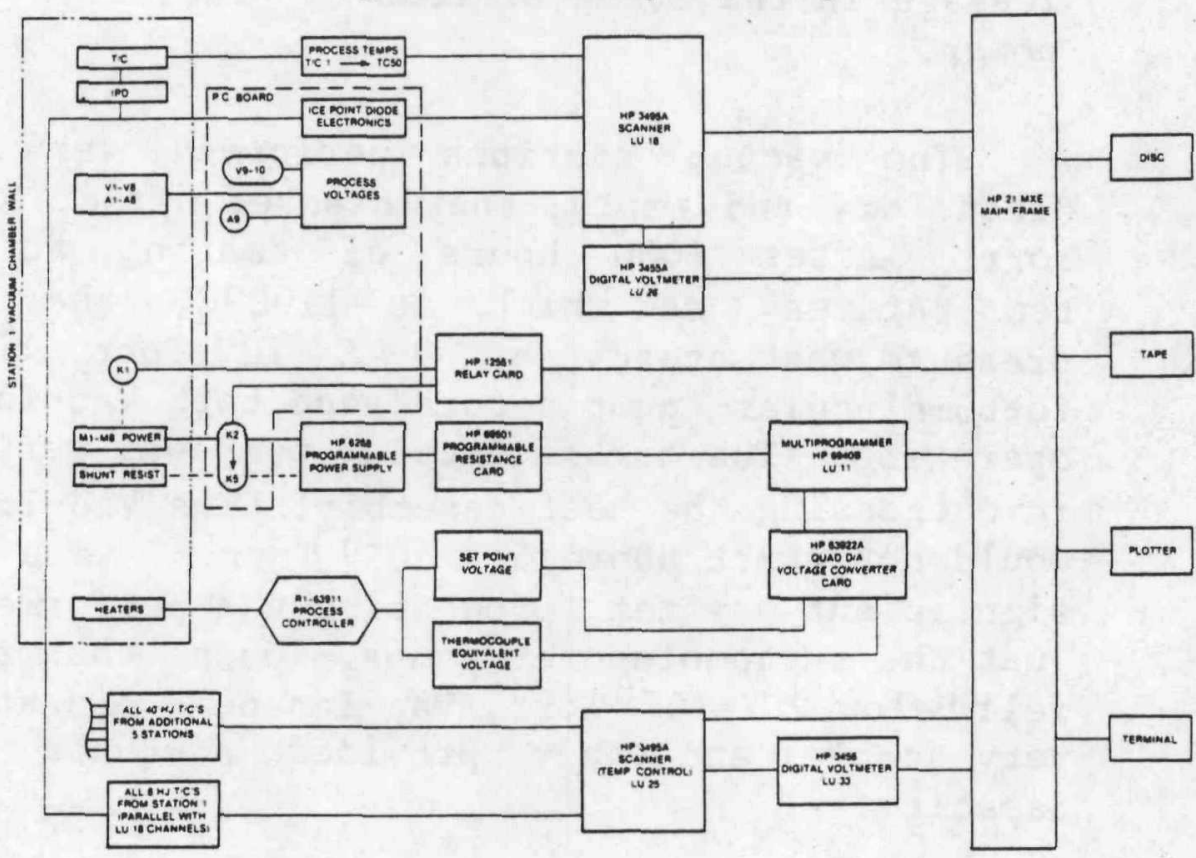




\section{Software}

\section{Functional Design}

The primary function of the control program during the conduct of MITG testing was the maintenance of a constant hot junction temperature. The functional description of the software providing dynamic control of the hot junction temperature is discussed in the following paragraphs.

a) Scanner Function. Each test assembly had 50 thermocouples installed, the general arrangement of which is shown in Figure 9. The millivolt output of these thermocouples was input to an HP $3495 \mathrm{~A} \mathrm{scanner.} \mathrm{The} \mathrm{software} \mathrm{control} \mathrm{of} \mathrm{the}$ scanner was performed over the Hewlett Packard Interface Bus. The scanner was first commanded to "listen" to a selected channel address and then the signal was output to a HP 3456 digital voltmeter.

Figure 9. General Arrangement of MITG Test Assembly Thermocouples

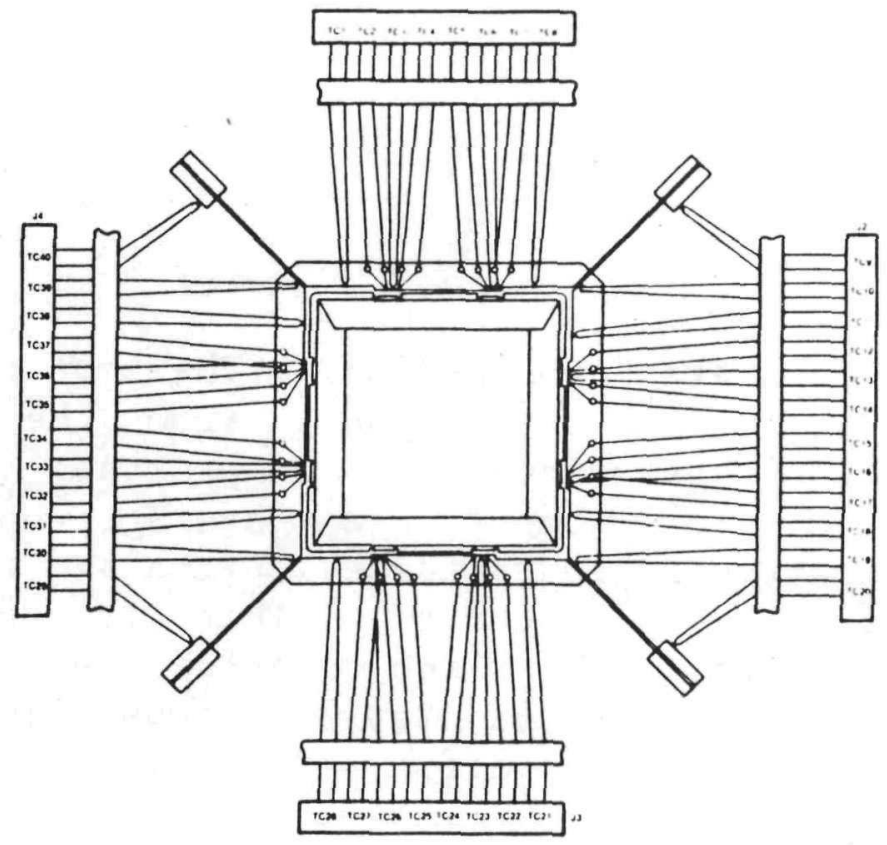


The DVM converted the analogue input to a digital output which is read by the program (SCAN). The functional design of the scanner program is given in Figure 10.

Figure 10. Scanner Functions

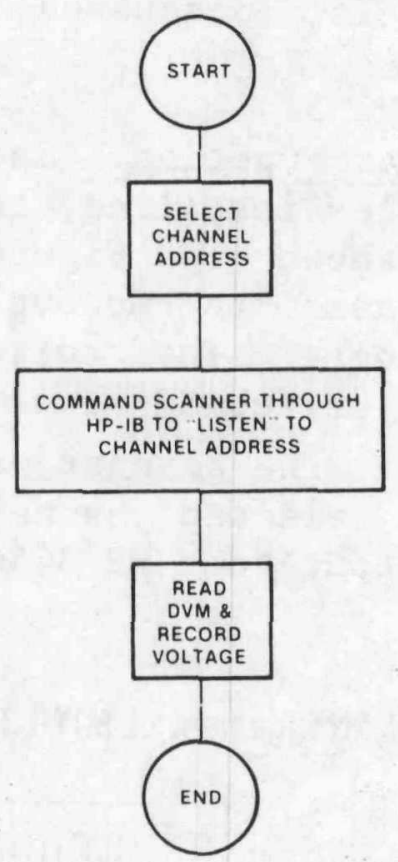

b) Averaging Function. The control scanner receives millivolt signals from the eight hot junction thermocouples. A program (MEAN) was written to compute an average hot junction temperature from these signals through an autioneering procedure. This is an important precautionary step which enabled the program to detect and discard any spurious signals due to multicouple damage etc. 
An arithmetic mean voltage was computed from the signals received from the eight hot junction thermocouples. Each individual signal was compared to the mean. Any signal which deviated more than 20 percent from the mean was discarded and a new average hot junction voltage was computed using the remaining signals. An appropriate error message indicating the time and the multicouple number thrown out of the auctioneering process was recorded.

The average millivolts, thus obtained, was converted to give an average hot junction temperature after correcting for the cold junction reference provided by the ice point diode sensors.

c) Flow Measurement Function. Heat was removed from the test assembly by the circulation of cooling water in the housing fins. Water from a constant temperature bath was circulated through the loop. In order to determine the heat rejected by the system, it is important to measure the temperatures of the inlet and outlet water very carefully. The critical nature of the rise in coolant temperature across the fin loop required the use of copper/constant differential thermocouples. The same was true for measuring the temperature rise in the coolant in the top and bottom covers. A program (FLOW) was written to convert the millivolt signals, obtained from the differential thermocouples, to the rise in coolant temperature across the fin loop.

The flow rate was determined by a signal (volts) delivered to the scanner from the flowmeter. A program was written to convert the volts into flow rate $(1 \mathrm{bs} / \mathrm{hr})$. Once the cooling water mass flow rate was determined and the temperature rise was known, the program computed the heat rejection in each of the coolant loops. 
The sum of these heat rejection rates and the module electric power output should equal the electric power intput to the heaters.Error in the comparison of those values indicates the accuracy of the heat balance, and by inference, may be used to determine the uncertainty in the observed system efficiency of the MITG slice.

d) Multiprogrammer Function. The power supply to the heater was controlled by RI 63911 process controller which in turn received its input analogue signals from the HP 6940B Multiprogrammer.

A quad D/A voltage converter card was installed in the multiprogrammer. The quad card contains 4 individually programmable 10-bit D/A voltage converter circuits that produce high speed bipolar output voltage proportional to the programmed digital data.

A program (QUADV) was written to drive the multiprogrammer. It addressed the quad voltage card in the multiprogrammer, selected the appropriate circuit which was loaded with digital data. This immediately activated the card to produce an output proportional to the programmed digital data. A functional design of the multiprogrammer program is shown in Figure 11 . 
Figure 11. Multiprogrammer Function

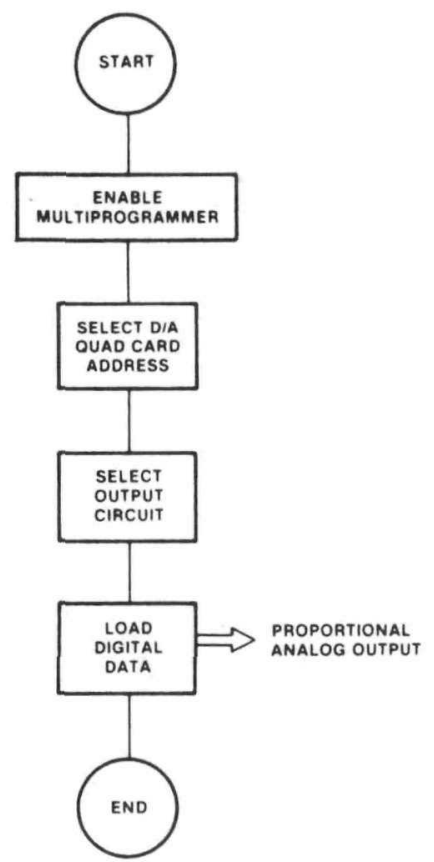

2. Detailed Design

The control program was activated every 30 seconds and was designed to perform the following functions.

a) Heatup/cooldown of hot junction.

b) Steady state operation.

c) Safety interlocks.

Each of these functions will be discussed in the following paragraphs.

a) Heatup/cooldown. The heatup or cooldown of the hot junction temperature should be executed at a carefully controlled rate. Large up or down transients in the temperature lead to unacceptably high thermal stresses which would very likely damage the multicouple. Programs were written which enabled the operator to heatup (HEATP) or cooldown (SHTDN) the hot junction at a specified controlled rate. 
The programs allowed the operator to enter

1. the rate (in degrees/hour) at which the heatup/cooldown should be executed, and

2. the final steady state temperature at which the hot junction should be maintained.

A time versus temperature ramp was set up to reflect the rate entered by the operator and the control program (CNTRL) was scheduled every 30 seconds.

During every execution of the control program, the following two signals were sent to the process controller through the quad D/A card:

1. A proportional voltage corresponding to the set point temperature along the time/temperature ramp and

2. A proportional voltage corresponding to the current hot junction temperature.

The process controller then sampled the two analogue signals. It increased/decreased the power to the heaters so that the hot junction temperature was set equal to the set point temperature.

b) Steady State. The heat up/cooldown proceeded automatically at the specified rate until the set point temperature was equal to the final temperature input by the operator. At this point in time, the control loop became a steady state operation with the temperature of the hot junction being maintained at the final temperature. It should be noted that the heatup/ cooldown or the steady state operations procedure could be interrupted at any time by the operator who could enter a new rate and final temperature. The control program then proceeded to increase/decrease the temperature of the hot junction at the new rate till steady state conditions were achieved. The functional design of the control program is given in Figure 12. 
Figure 12. Control Function Safety Interlocks

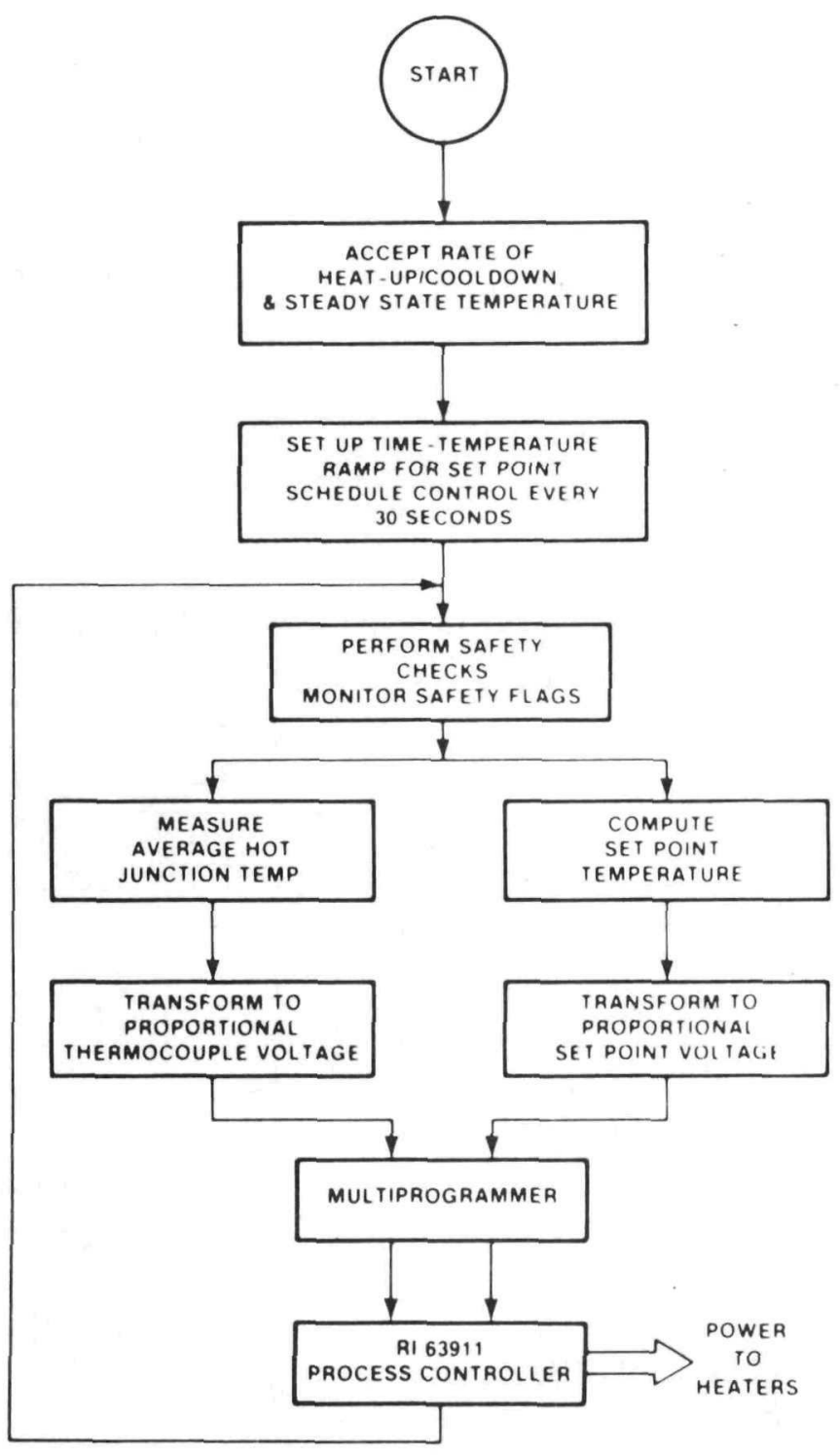


c) Safety Interlocks. During the execution of the control loop (Figure 13), a number of safety interlocks were incorporated to shut down the heater power if prescribed limits on the following variables were exceeded:

1. High hot junction temperature $\left(>1100^{\circ} \mathrm{C}\right)$

2. High cold junction temperature $\left(>325^{\circ} \mathrm{C}\right)^{\prime}$

3. Low flow rate of cooling water $(<10 \mathrm{lbs} / \mathrm{hr})$

4. High bell jar pressure (>10-4 torr)

Figure 13. MITG Test Assembly Control and Safety Functions

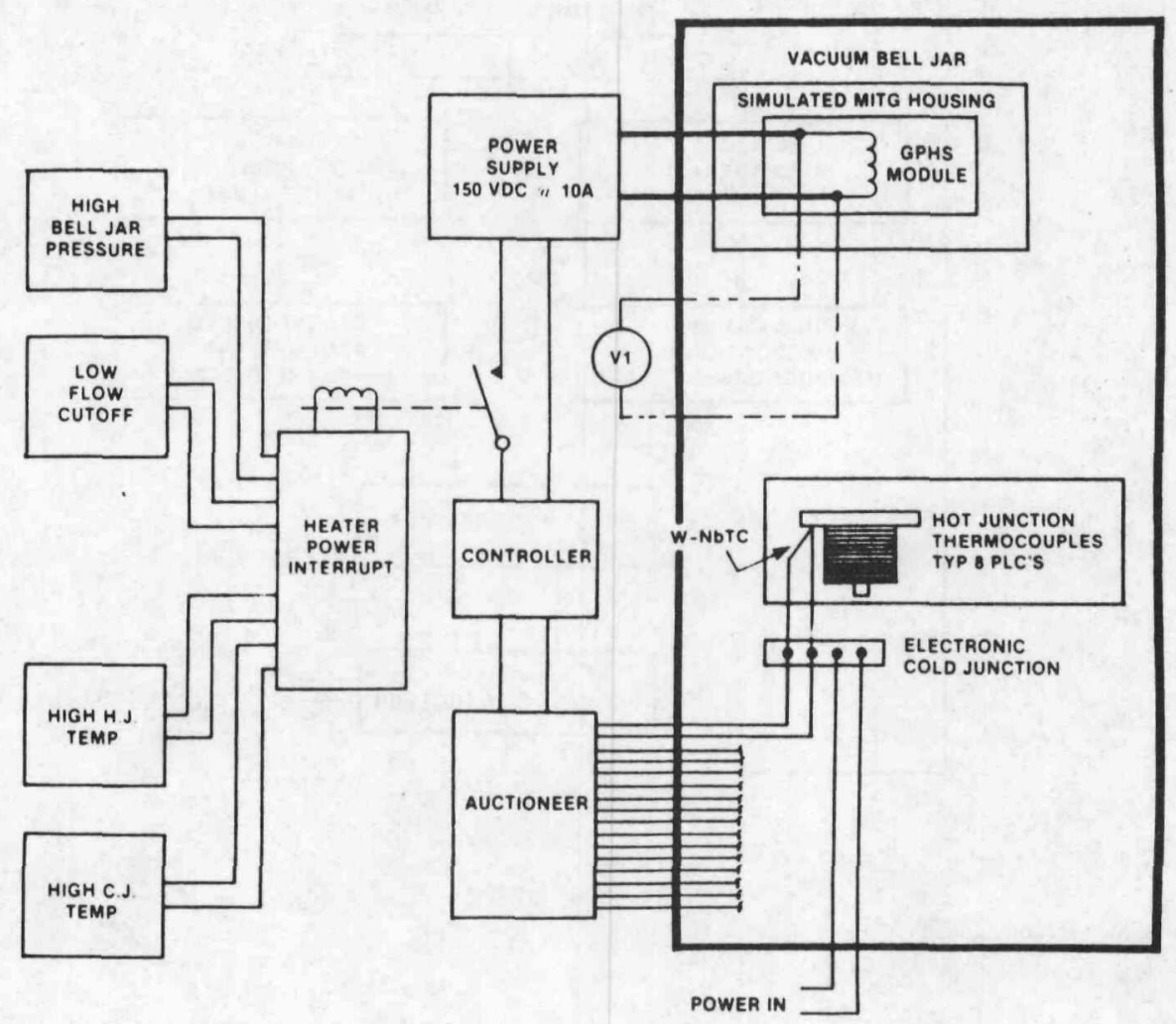


A safety relay was provided to control the power to the heaters. Normally, this relay was closed, but in the event of any one of the safety flags being set, the following actions were performed by the safety program:

1. The safety relay was opened so that the power to the heater was turned off.

2. The set point voltage was gradually set to zero volts along a cooldown ramp.

3. The time of occurrence and appropriate safety alarm message was recorded.

The control loop halted stepping the set point voltage up or down the set point ramp. The program entered a safety mode of operation wherein the safety flags were continuously monitored. As soon as all the safety alarms were cleared, the control loop measured the current hot junction temperature and proceeded to step it up the set point ramp at the specified rate.

This mode of recovery from a safety alarm ensures that large up/down transients of the hot junction temperature were avoided, minimizing any damage to the multicouple.

Since the occurrence of any of the above safety alarms caused the heater to be turned off, it was important to monitor the rate of cooling of the hot junction. If the rate was too high $\left(>100^{\circ} \mathrm{C} /\right.$ hour $)$ and the temperature of the cold junction was less than $325^{\circ} \mathrm{C}$, the safety function caused the cooling water flow to be stopped. If high hot junction or cold junction temperatures were sensed, the cooling water flow was restarted immediately. 


\section{Data Acquisition}

A program was written to acquire temperature and electrical data from each test assembly. This was scheduled to be activated every 10 minutes. The raw data (mV), the converted data (degrees $\mathrm{C}$ ), and the open circuit module voltages were stored in a time sequenced format on magnetic tape.

a) Temperature Measurements. Each test assembly had 50 thermocouples installed. The general arrangement of these was shown in Figure 9.

A listing of temperature instrumentation provided to obtain the required test information is as given below.

The values shown in parenthesis are the number of places the temperature is monitored in each test assembly and the expected range of variation in that temperature.

1. Multicouple hot junction temperature. (8 places, $900^{\circ}-1150^{\circ} \mathrm{C}$ )

2. Multicouple cold junction temperature. ( 8 places, $200^{\circ}-400^{\circ} \mathrm{C}$ )

3. Multicouple cold stud housing interface temperature. ( 8 places, $200^{\circ}-400^{\circ} \mathrm{C}$ )

4. MITG housing segment face temperature. ( 8 places, $200^{\circ}-400^{\circ} \mathrm{C}$ )

5. MITG housing segment fin root temperature. ( 4 places, $\left.200^{\circ}-400^{\circ} \mathrm{C}\right)$

6. Cooling water saddle temperature. (4 places, $200^{\circ}-400^{\circ} \mathrm{C}$ ) 
7. Simulated GPHS module temperature.

( 2 places, $1200^{\circ}-1400^{\circ} \mathrm{C}$ )

8. Top cover temperature. (2 places, $\left.40^{\circ} \mathrm{C}\right)$

9. Bottom cover temperature. (2 places, $40^{\circ} \mathrm{C}$ )

10. Fin cooling water inlet temperature. (1 place, $20^{\circ} \mathrm{C}$ )

11. Fin cooling water outlet temperature. (1 place, $20^{\circ} \mathrm{C}$ )

12. Top and bottom cover cooling water inlet temperature. ( 1 place, $\left.20^{\circ} \mathrm{C}\right)$

13. Top and bottom cover cooling water outlet temperature. ( 1 place, $\left.20^{\circ} \mathrm{C}\right)$

The signals were corrected for the cold junction reference temperature obtained from the ice point diode electronics and converted to temperature using mV-temperature calibration for $\mathrm{W} / \mathrm{Nb}$ thermocouples.

The cooling water temperature measurements were made with copper/constant differential thermocouples. These signals were converted to temperatures using the appropriate algorithm for a TYPE T thermocouple. 
b) Electrical Measurements . The program measured the following electrical outputs.

1. The eight individual multicouple output voltages.

2. The series connected module voltage.

3. The shunt power supply voltage.

4. The voltages across the high precision current measuring resistors in series with the multicouples and the foil insulation circuit. These voltages were used to compute the individual multicouple shunt currents.

c) Shunt Degradation Measurements. Shunt degradation as a result of silicon sublimation at the hot end is an important life limiting mechanism in present SiGe RTGs. The silicon condenses in a low temperature zone and the resultant deposit acts as a conductive shunt between the thermoelectric legs and the multifoil insulation. The consequent shunt currents cause unacceptable power output degradation in the generator, unless the silicion sublimation can be eliminated or significantly retarded. In the thermoelectric module design used in the MITG, the role of sublimation supressant is played by a relatively thick (.003 inch) coat of mulite glass. The following test which was performed once a day was designed to evaluate the efficacy and the long term stability of the coating.

Individual shunt characteristics for each module were measured by using a ganged 8-pole double throw relay which simultaneously broke the series connections between the eight multicouples and shorts each one. The eight shorted multicouples were connected to a common terminal of an external supply through individual high precision current measuring resistors Rl-R9 (Figure 14). The other terminal of the power supply was connected to the foil package. By this arrangement, the individual shunt characteristics for the eight multicouples could be measured simultaneously. The functional design of the shunt program is discussed below. 
Figure 14. MITG Test Assembly Wiring Diagram

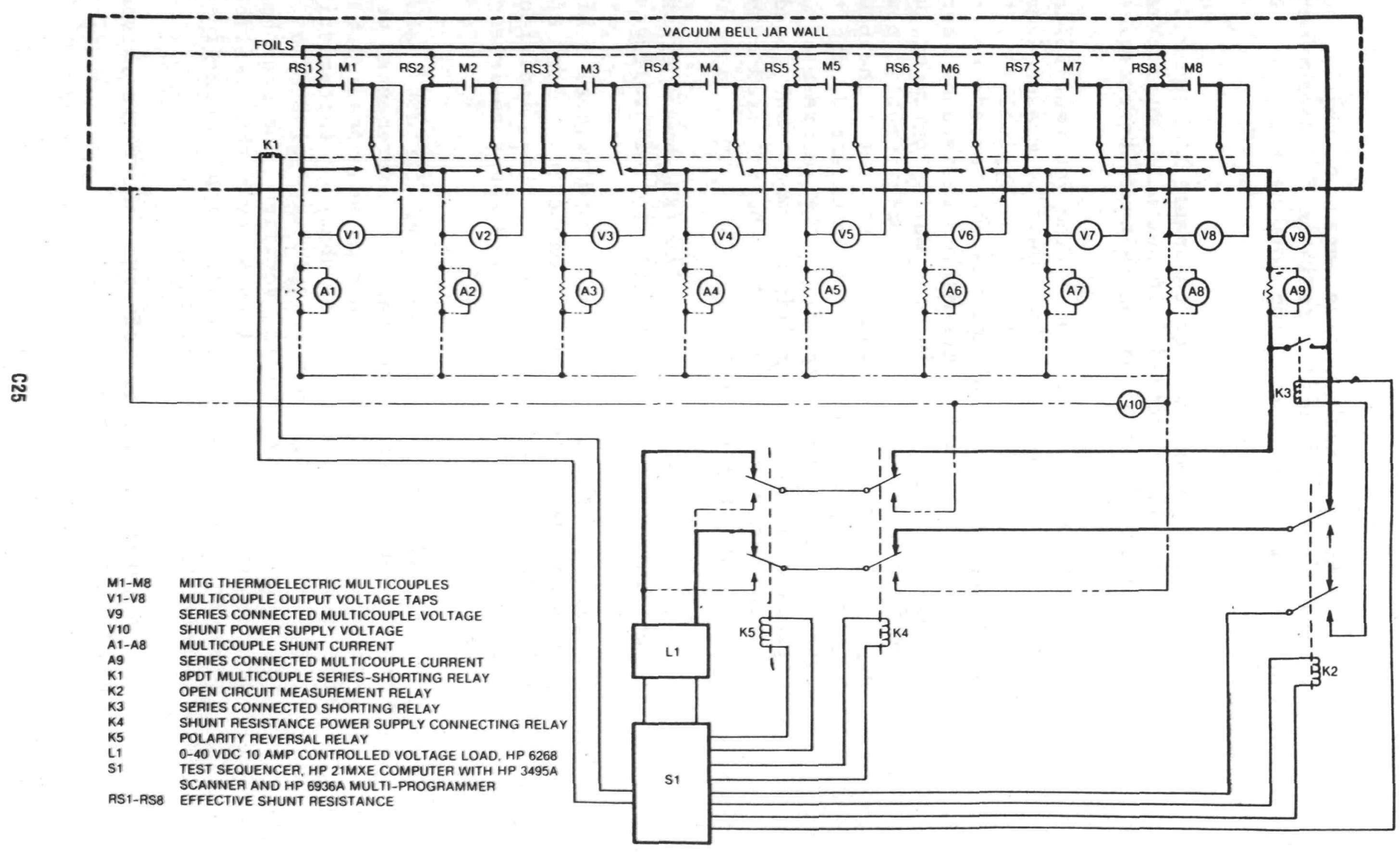


1) Relay Function. The open circuit, short circuit and shunt resistance measurements necessitate the energizing of relays on the HP 12551 relay card.

A program was written to enable the individual relays to be energized. It set up an appropriate bit pattern depending on the relay to be energized.

2) Resistance Function. The current-voltage characteristic of each shunt must be measured over a range of load voltage. Typically, the multicouple to foil voltage must be varied from +30 to -30 volts. The programmable resistance card, HP 69501A, installed in the multiprogrammer, was used in conjunction with the programmable power supply to produce output voltages (of the power supply) proportional to the programmed digital data. The program selected the address of the resistance card in the multiprogrammer and loaded it with data which generated the required output voltage on the power supply.

The polarity reversal relay (K5) was energized to generate negative multicouple to foil voltages.

The voltages across the individual high precision resistances were measured. For each value of the load voltage, the individual multicouple shunt currents and shunt resistances were computed.

All the data from the open circuit, short circuit and shunt resistance measurements were stored on magnetic tape in a time sequenced format for post processing. The shunt characteristics (IV) curves for each multicouple were plotted as a function of time. This verified the non-ohmic nature of the shunts formed by the silicon condensate and the resultant highly non-linear current-voltage characteristics.

A detailed design of the shunt resistance function is shown in Figure 15. 
Figure 15. Shunt Resistance Function

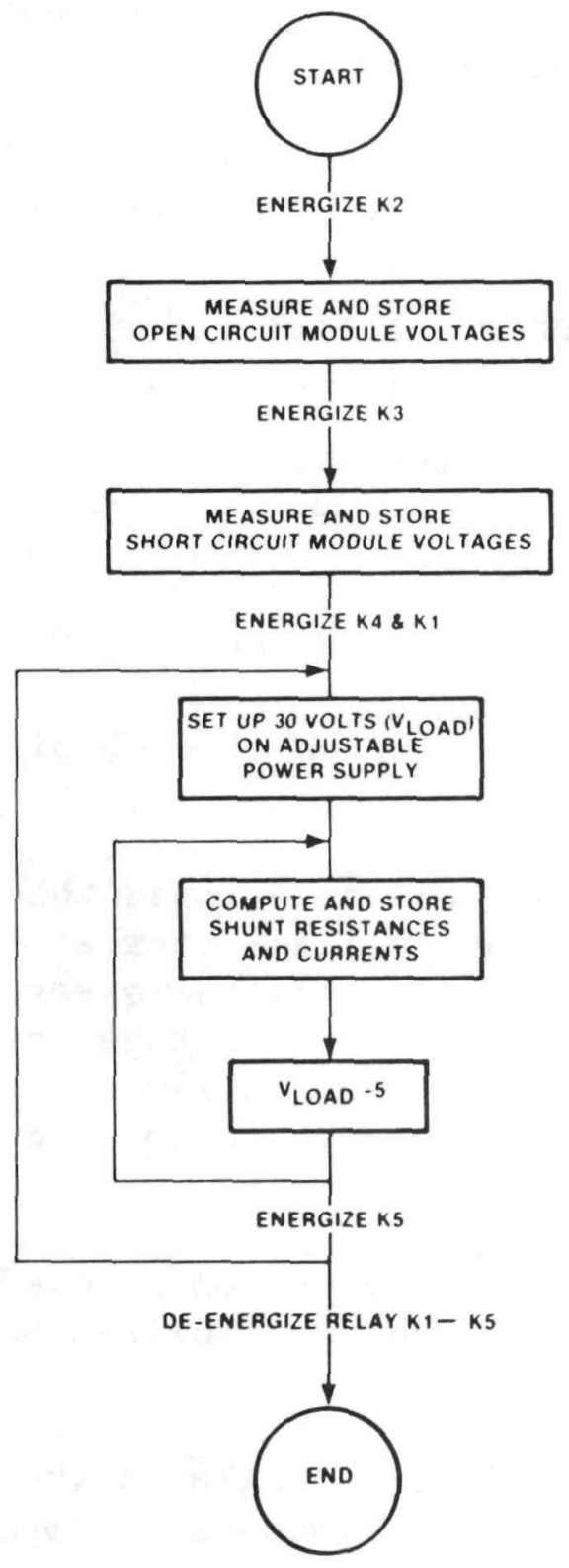




\section{Check-out Software}

A program, DOCTR, was written to perform system diagnostics. This program was used as a tool to check out the installation of the test assemblies.

DOCTR assisted the user in verifying the wiring for the control and instrumentation in a systematic manner.

The program presented the user with the following options for verification:

1. Scanner function

2. Power Supply function

3. Control function

4. Relay function

5. Safety function

6. Shunt Calibration

A brief description of each of these options is given below.

The scanner option allowed the user to scan and measure the voltage across each of the 80 channels, and an appropriate message indicating the channel number and the voltage across it appeared on the screen. The millivolts from the 50 thermocouples were also converted into temperatures.

The power supply option allowed the user to generate a user defined voltage on the adjustable power supply.

The control option allowed the user to enter set point and thermocouple temperatures and to determine the voltage generated at the RI 63911 process controller. 
The relay option allowed the user to open or close individual relays or a combination of relays on the HP 12551 relay card.

The safety option allowed the user to determine whether a safety alarm caused the safety relay to be opened.

The safety features which could be tested were :

1. High hot junction temperature.

2. High cold junction temperature.

3. Low flow rate of coolant.

4. High rate of cooling of hot junction.

The shunt calibration option allowed the user to calibrate selected shunt resistances with respect to the actual measured shunt resistances for different values of the load voltage.

\section{Post-Processing Software}

All the data acquired during the test were stored on magnetic tape in a time sequenced format. Post-processing programs were written to read the tape and extract the following data values as a function of elapsed time for each multicouple:

1. Multicouple open circuit voltage.

2. Hot junction temperature.

3. Cold junction temperature.

The Seebeck coefficient was extracted using the measured multicouple hot and cold junction temperatures and the open circuit voltage. Each of these variables was plotted as a function of elapsed time. Typical plots are shown in Figures 3 and 4 . 


\section{Test Operations}

Test operations were conducted continuously as planned. Some control and data acquisition system bugs were detected and corrected; however, the software took much of the drudgery out of running the tests and obtaining and reducing data. In addition, the system diagnostic program, DOCTR, was very useful in checking out the installation of the test assemblies.

\section{Installation}

The multicouples were inspected when from Syncal. A resistance check was compared to the Syncal measurements.

received made and personnel installed the multicouples in the prototypical insulated housing and Fairchild personnel installed and instrumented the test assembly in the vacuum chamber. The control and instrumentation wiring had already been installed and rung out using (DOCTR) up to custom built terminal strips inside the vacuum chamber. Specially built teflon terminal strips were used because of outgassing problems encountered with standard "low outgassing" terminal strips.

After installing the test assembly in the chamber, all internal instrumentation and power wiring was completed, the cooling water lines were connected and leak checked, the module internal pressure monitoring ionization gauge tubulation was installed and the chamber was closed. The system was then roughed with the turbomolecular pump and leak checked. The chamber, if leak tight, would reach the $10^{-4}$ Torr scale in 25 minutes. Pump down time and the pitch of the turbomolecular pump were very good indicators of system leak tightness. 


\section{Heat-Up}

The electric heaters (and foil insulation packages) were manufactured by Thermo Electron Corporation (TECO). These heaters were fabricated from HT molybdenum wire. This material is very oxidation sensitive, and TECO provided an outgassing temperature schedule which severely constrained the system heat-up rate. Although Syncal allowed a $100^{\circ} \mathrm{C} /$ hour heat-up rate, the TECO limitations on heater temperature and pressure stretched the heat-up period to approximately 600 hours. The chamber pressure rarely rose above $2 \mathrm{X}$ $10^{-6}$ Torr while the test assembly was outgassing. The module internal pressure varied from $1 \times 10^{-4}$ to $5 \times 10^{-6}$ Torr during the outgassing period. The extreme limitations on heat-up rate caused by the heater pressure limitation was the basis for a system redesign. Future system test assemblies will be fitted with a removeable plug in the top foil insulation pack. During outgassing operations, this plug will be lifted to improve the conductance between the module internals and the vacuum chamber. This, and the removal of the internal pressure monitoring ionization gauge, should greatly increase the rate at which the test assembly can be processed.

The fact that there were problems with the multicouples first became evident during the assembly heatup testing phase. The wealth of instrumentation and the ease with which the data could be reduced was very helpful in determining the system test program direction. 


\section{Steady State}

Steady state operations were conducted with only the Vac-Ion pumps operating and all system isolation valves closed. These operations were routine and were completely under computer control. Post-processing of these data produced easily interpreted figures which were very useful in assessing the condition of the individual multicouples. A pattern emerged which gave credence to the theory that a common mode failure had occurred and that nothing would be gained by continuing the test. If the individual multicouples could not have been isolated and their performance measured, the existence of this common mode failure could not have been inferred.

\section{Coo1-Down}

The multicouples were cooled down using the cool-down program SHTDN. The down ramps were interrupted at specific temperatures to make open circuit, internal resistance and shunt resistance measurements. These data were also post-processed using the software developed for the test program. No problems were encountered during this period until the failure of the HP 3456 DVM. This failure locked the HP-IB bus and prevented the heater controllers from receiving updated temperature information from the computer. At the time of failure, one controller was supplying power above the set point and one was below. As a result, one system continued to heat and the other continued to cool.

A system redesign has added an independent noncomputer-related override to prevent exceeding a pre-selected temperature. The RI 63911 heater controllers have a power limiter which indirectly provides this function. When operating below the design temperature, the power limiter has insufficient range to control the heater power; although it will prevent the design temperature being exceeded, rapid ramps to the design value can be encountered. The independent safety system will preclude these ramps. 


\section{RESULTS}

The test durations for the several MITG builds are summarized in Table $I$. The tests were terminated because of the high observed internal resistance measured in all multicouples. A snapshot of the parameters measured in the SiGe module is shown in Figure 16. Table II shows a summary of the before and after test multicouple resistances. A plot of internal resistance vs temperature of a standard SiGe multicouple is shown in Figure 17. This multicouple (Station 1, Build 2, Multicouple 8) was the best behaved in terms of output power and internal resistance of all the tested multicouples. A plot of the output voltage vs time for this multicouple is shown in Figure 18 . The multicouple was at design temperature $\left(1000^{\circ} \mathrm{C}\right.$ $\mathrm{HJ}, 300^{\circ} \mathrm{C} \mathrm{CJ}$ ) during the period 500 to 720 hours. Controlled heatup and cooldown ramps were used to reach this temperature.

Table I. Test Duration Summary

\begin{tabular}{|c|c|c|}
\hline \multicolumn{2}{|r|}{ STATION 1 - STANDARD SIGe } & STATION 2 - GaP DOPED SIGe \\
\hline BUIL & & BUILD \\
\hline \multirow[t]{2}{*}{1} & 29 JUNE $82-2$ JULY 82 & 9 AUG 82 - 28 SEPT 82 \\
\hline & $\begin{array}{l}\text { MAXIMUM HOT JUNCTION TEMP } \sim 420^{\circ} \mathrm{C} \\
\text { TIME AT TEMP }<10 \text { HOURS } \\
\text { TOTAL TEST TIME }<70 \text { HOURS }\end{array}$ & $\begin{array}{l}\text { MAXIMUM HOT JUNCTION TEMP } 1000^{\circ} \mathrm{C} \\
\text { TIME AT MAX TEMP }>200 \text { HOURS } \\
\text { TOTAL TEST TIME }>1100 \text { HOURS }\end{array}$ \\
\hline \multirow[t]{2}{*}{2} & $17-31$ JULY $82 ; 9$ AUG $82-28$ SEPT 82 & \\
\hline & $\begin{array}{l}\text { MAXIMUM HOT JUNCTION TEMP } 1000^{\circ} \mathrm{C} \\
\text { TIME AT MAX TEMP > } 200 \text { HOURS } \\
\text { TOTAL TEST TIME }>960 \text { HOURS }\end{array}$ & \\
\hline
\end{tabular}

Table II. Multicouple Resistance

\begin{tabular}{|c|c|c|c|c|}
\hline \multirow{3}{*}{ MULTICOUPLE E } & \multicolumn{2}{|c|}{ STATION 1 } & \multicolumn{2}{c|}{ STATION 2 } \\
\cline { 2 - 5 } & $\begin{array}{c}\text { RESISTANCE } \\
\text { AS INSTALLED }\end{array}$ & $\begin{array}{c}\text { RESISTANCE } \\
\text { AFTER TEST }\end{array}$ & $\begin{array}{c}\text { RESISTANCE } \\
\text { AS INSTALLED }\end{array}$ & $\begin{array}{c}\text { RESISTANCE } \\
\text { AFTER TEST }\end{array}$ \\
\hline & $\Omega$ & $\Omega$ & $\Omega$ & $\Omega$ \\
1 & 48 & 1405 & 82.0 & 332.0 \\
2 & 30 & 1103 & 24.0 & 241.0 \\
3 & 303 & 1013 & 41.0 & 120.0 \\
4 & 34 & 72 & 10.3 & 321.0 \\
5 & 121 & 33 & 9.7 & $\propto$ \\
6 & 41 & 41 & 9.8 & 10.4 \\
7 & 1776 & 997 & 41.7 & 96.0 \\
8 & 124 & 36 & 110.0 & .24 \\
\hline
\end{tabular}


Figure 16. Station 2 Temperature Distribution

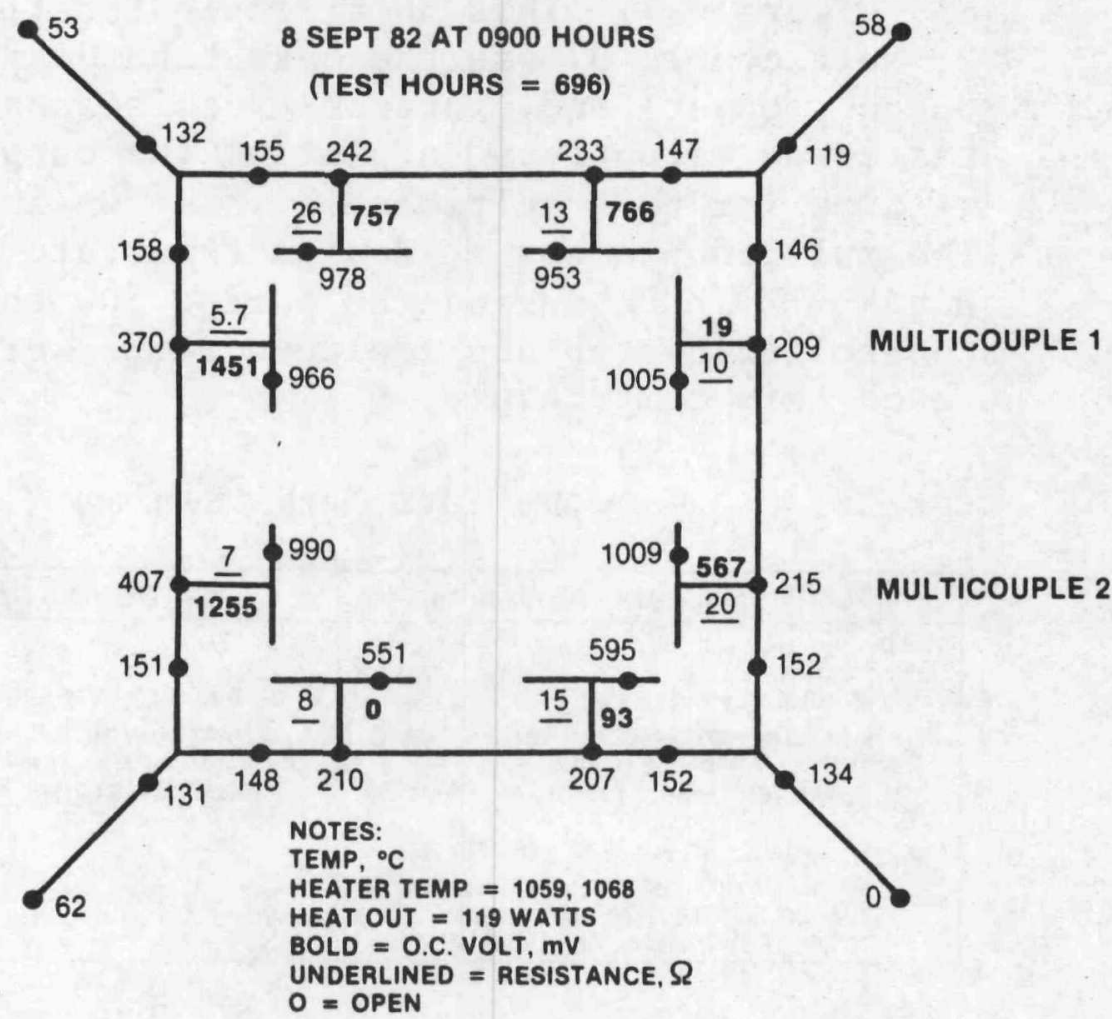


Figure 17. Multicouple 8 Internal Resistance - Temperature History

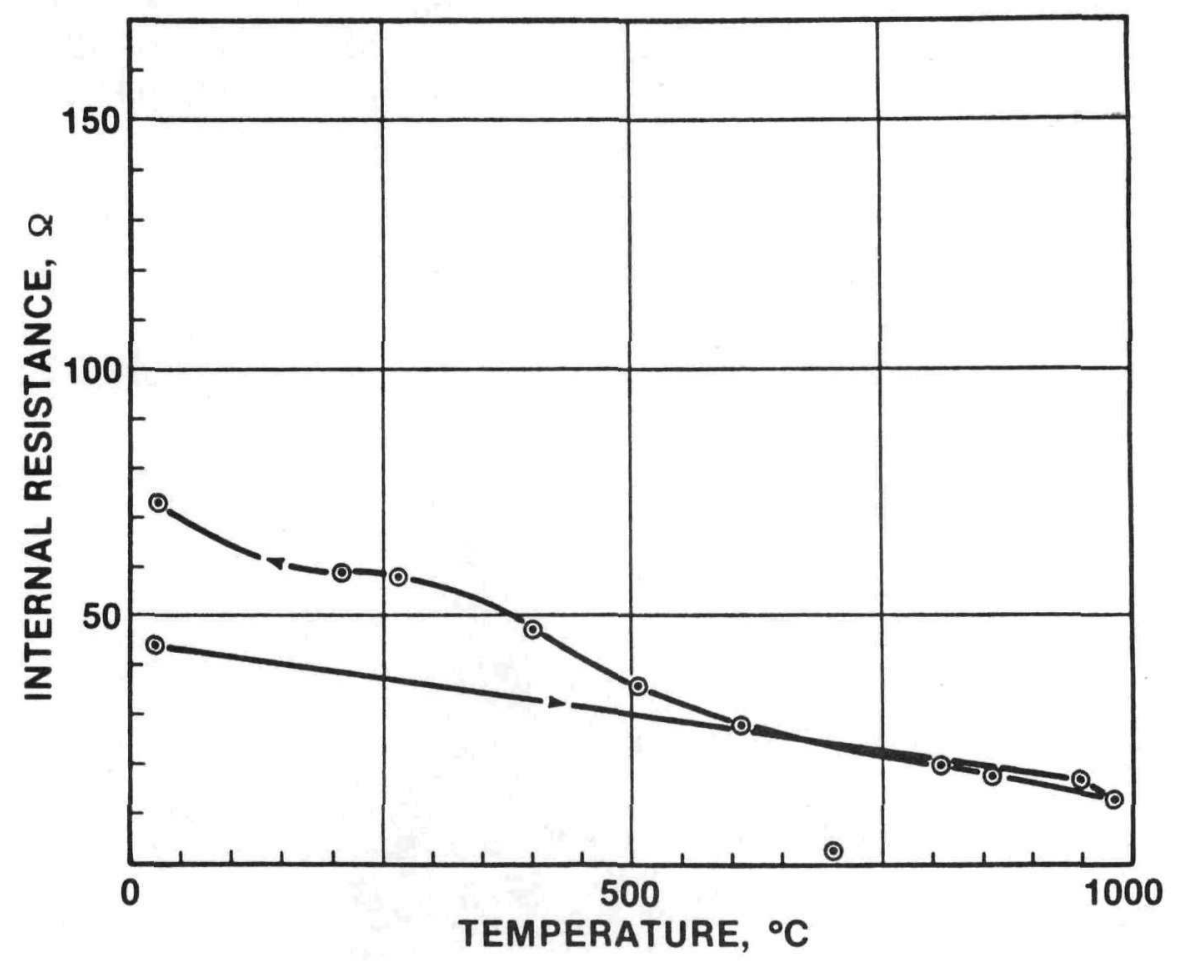

Figure 18. Multicouple Output Voltage

STATION 1 MULTICOUPLE 8

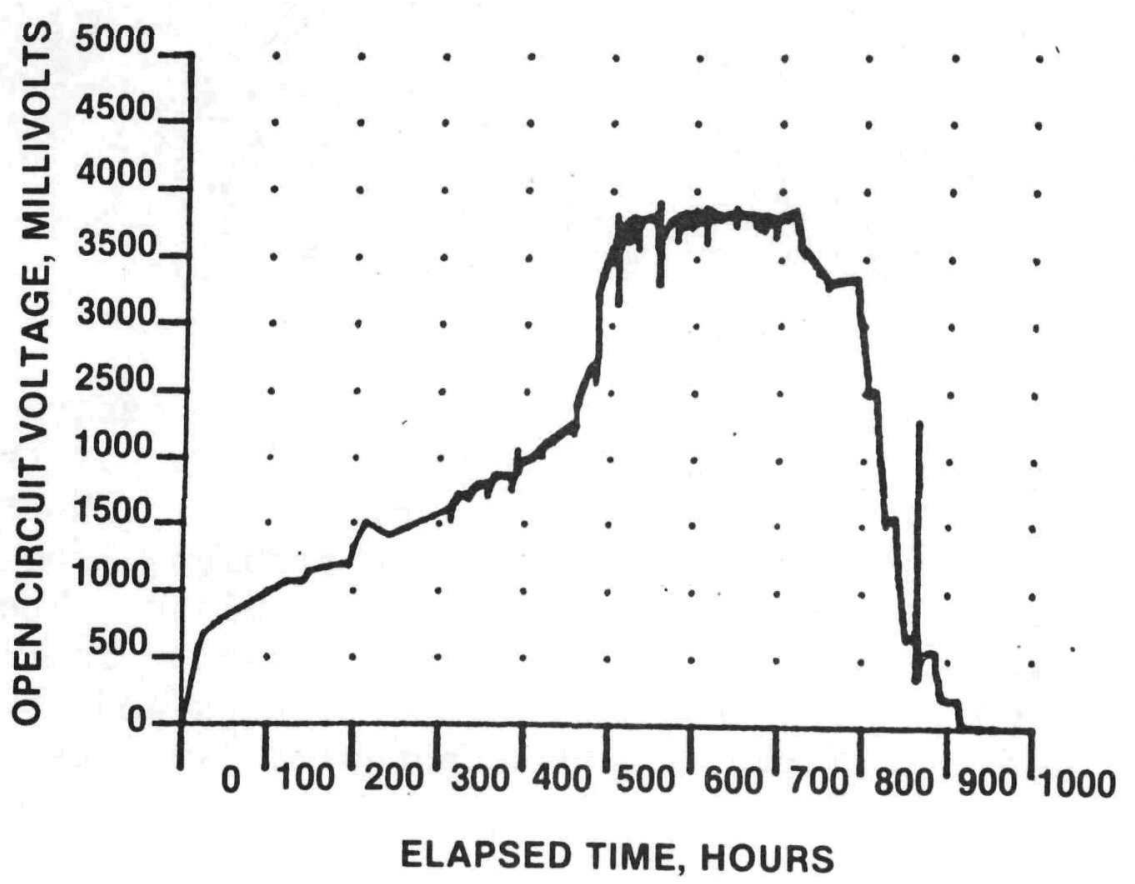


Examination of the multicouples removed from all builds showed cracking at the cold stud-SiGe interface. This cracking was predominantly in the SiGe, not in the glass. Figure 19 shows a typical cracked module.

Figure 19. Photograph of a Cracked Multicouple

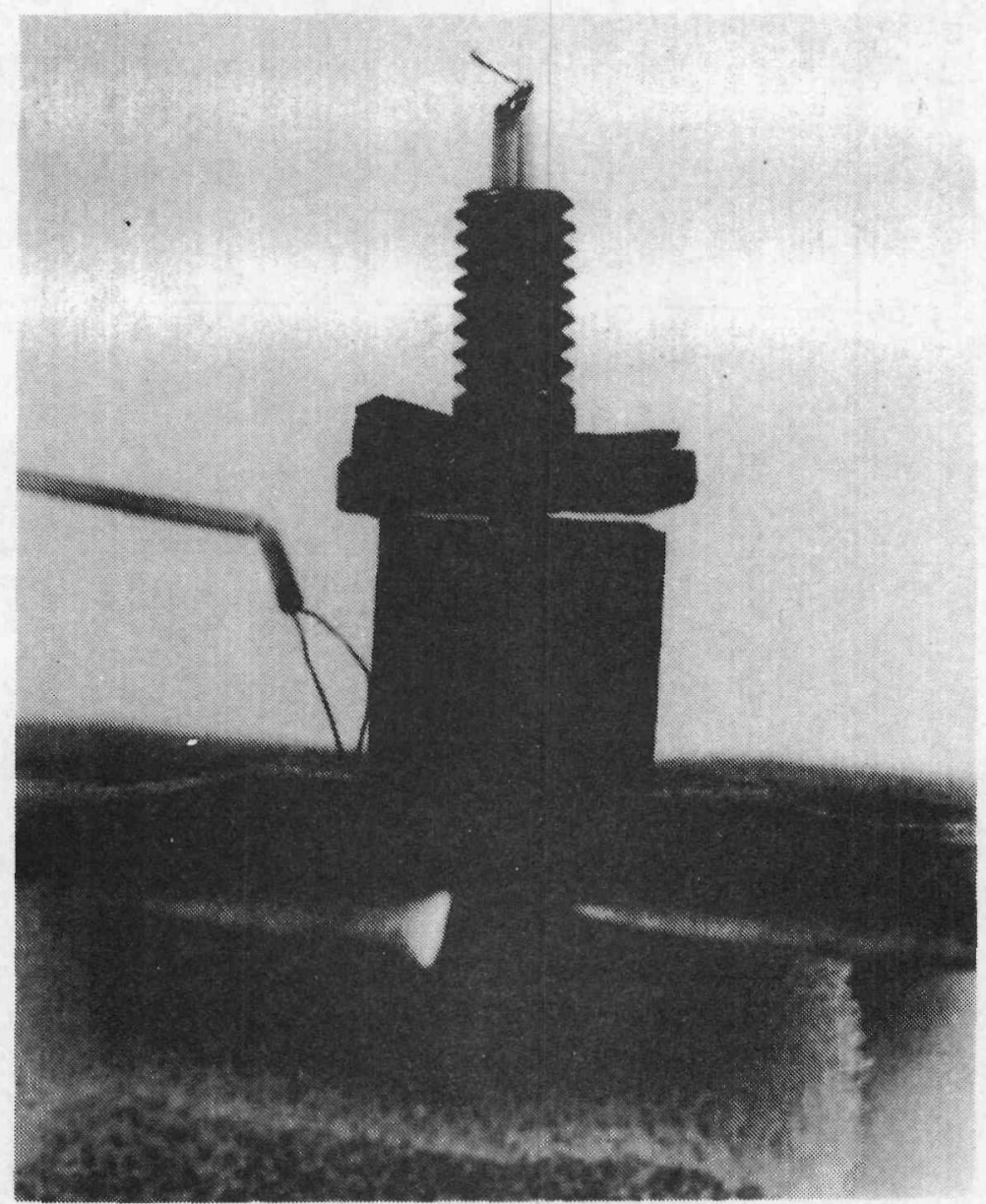

Detailed metallographic examination of the multicouple hot end showed that there was incomplete glass coverage of the hot shoes. This led to a diffusion of germanium from the shoe bonding sites to the intra-shoe glass. The change in shunt resistance which is shown in Figures 20 and 21 resulted from volatilization and deposition of germanium through porosity in the hot shoe protective glass. 
Figure 20. Shunt Resistance Station 1

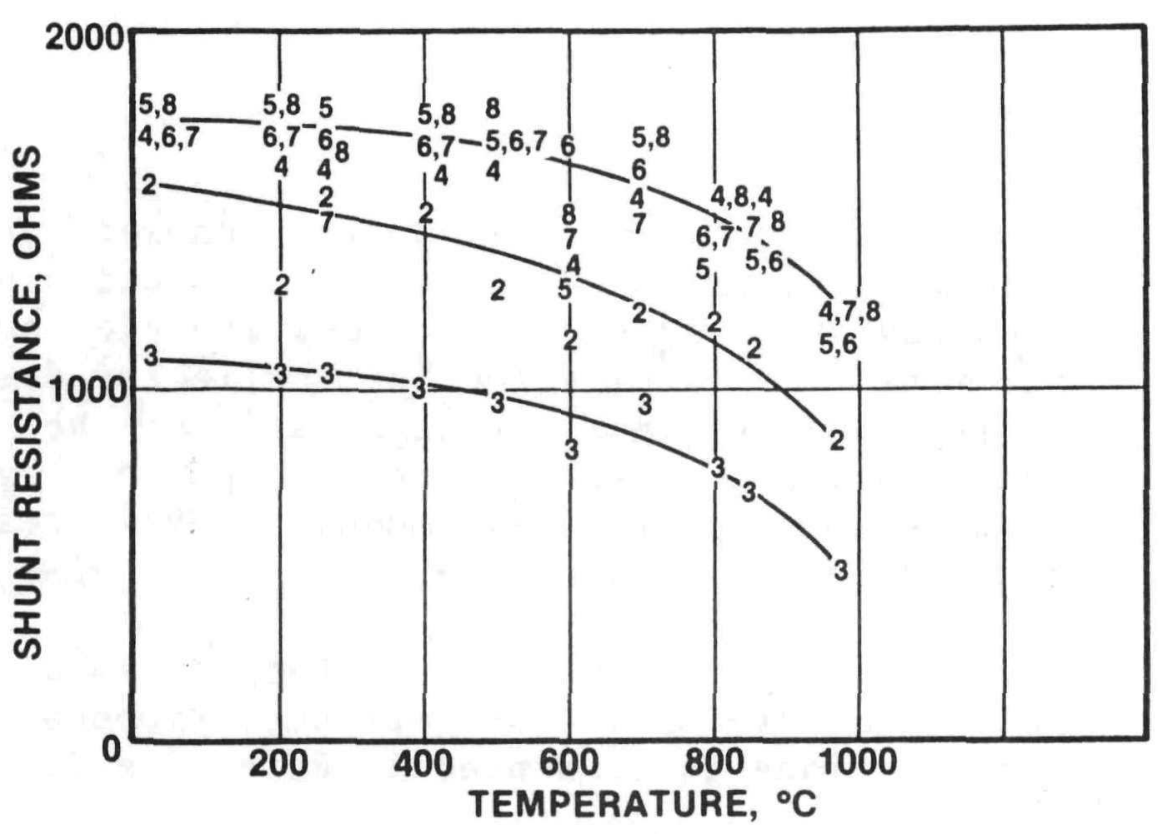

Figure 21. Shunt Resistance Station 2

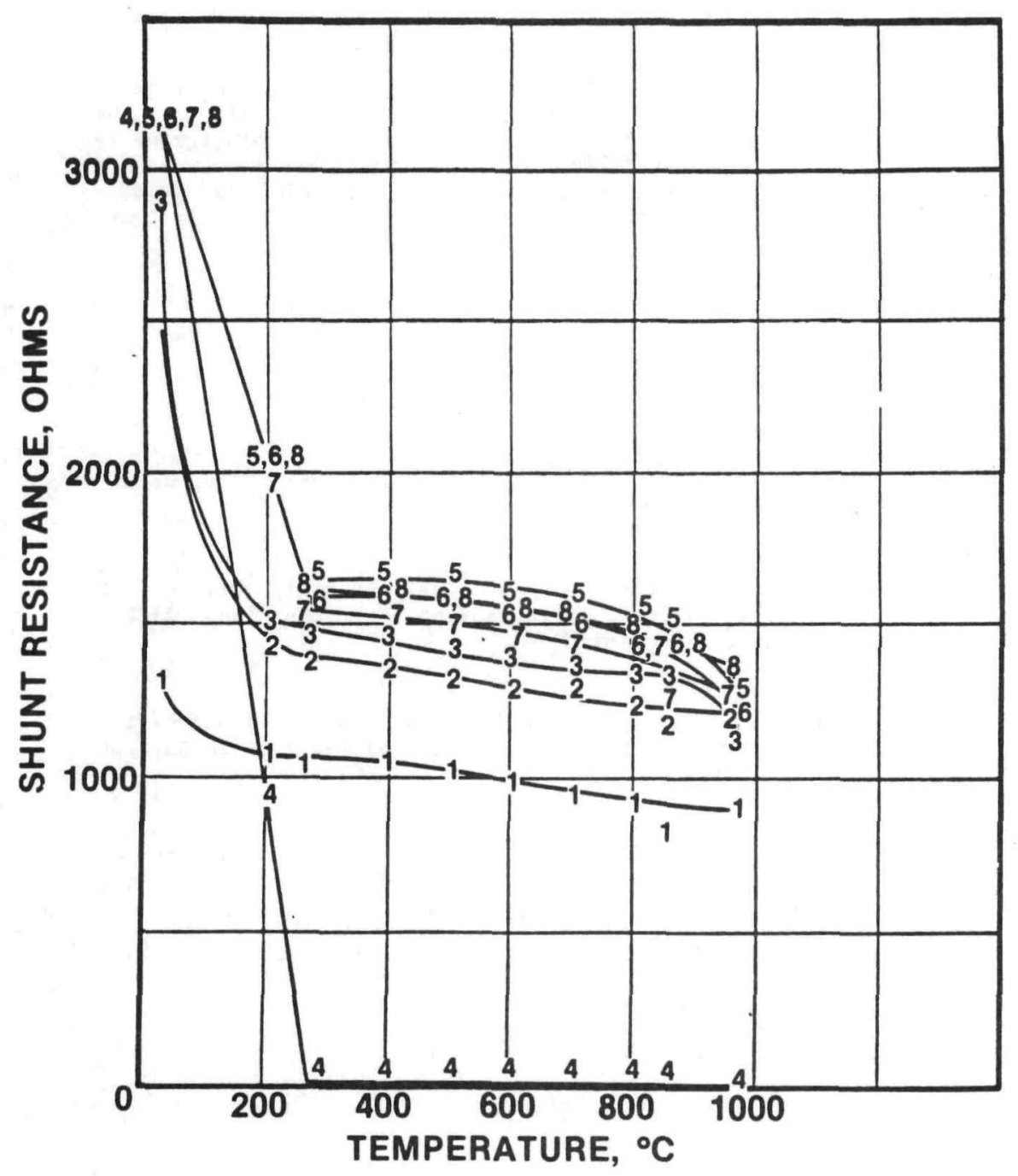


In general, the post mortem showed what was inferred from the test data. Steps are now underway to redesign the multicouple cold end hardware, to modify the heat collector design and to improve the glass coverage at the hot shoes. Additional test results at the component level have been encouraging. New module level tests are planned for September 1983.

A detailed discussion of the analysis of the cold end cracking and of the proposed design modifications is presented in Reference 2.

\section{Acknowledgment}

The authors take pleasure in acknowledging
that the work described here reflects the
contributions of many individuals, including
(alphabetically): L. Jarvis, and A. Schock of
Fairchild; V. Raag and L. Waddingham of Syncal,
and J. Hardy of TECO.

\section{References: (Part C):}

Part:

A. [1] A. Schock, "Modular Isotopic Thermoelectric Generator", Proceedings of the 1981 IECEC, Volume 1, Pg. 327.

B. [2] A. Schock, "MITG Test Assembly Design and Fabrication", Proceedings of the 1983 IECEC, preceding paper.

D. [3] A. Schock, "MITG Post Test Analysis and Design Improvements", Proceedings of the 1983 IECEC, next paper.

E. [4] A. Schock, "Revised MITG Design, Fabrication Procedure, and Performance Predictions", Proceedings of the 1983 IECEC following paper. 


\title{
Part D:
}

\section{MITG Post-Test Analysis and Design Improvements}

\author{
A. Schock \\ Fairchild Space Company \\ Germantown, Maryland 20874
}

(Presented at the 1983 Intersociety Energy Conversion Engineering Conference) 


\section{Abstract}

The design, performance analysis, and key attributes of the Modular Isotopic Thermoelectric Generator (MITG) were described in a 1981 IECEC paper; and the design, fabrication, and testing of prototypical MITG test assemblies were described in preceding papers in these proceedings. Each test assembly simulated a typical modular slice of the flight generator.

The present paper describes a detailed thermal-stress analysis, which identified the causes of stress-related problems observed during the tests. It then describes how additional analyses were used to evaluate design changes to alleviate those problems. Additional design improvements are discussed in the next paper in these proceedings, which also describes revised fabrication procedures and updated performance estimates for the generator. 
1. Introduction

The design of the Modular Isotopic Thermoelectric Generator (MITG), consisting of identical modular generator slices, was described [1] by the author at the 1981 Intersociety Energy Conversion Engineering Conference (IECEC). Since it offered a number of desirable features and a much higher power-to-weight ratio than current RTGs, DOE initiated a program to build and test such prototypical generator slices, primarily to measure their power output and efficiency, and to determine their performance reproducibility and long-term stability.

The design and fabrication of the initial test assemblies are discussed in a previous paper [2] in these proceedings; and the test plan, methodology, instrumentation, computer control and data acquisition systems, and initial test results are described in the preceding paper [3].

The present paper describes a serious thermalstress problem revealed by the tests, and presents a detailed structural analysis which identified its causes. Based on the understanding gained, the paper then discusses a number of design modifications, and presents analyses demonstrating their effectiveness in relieving the observed thermal-stress problem.

In addition, the next paper in these proceedings [4] describes some alternative design changes for eliminating the observed thermal-stress problems, and for improving thermal conductance and hermetic sealing between the generator housing and the multicouple cold ends. These design changes have been validated analytically, and are now undergoing experimental confirmation.

Finally, based on these design changes and on updated thermoelectric properties measured at various laboratories during the past two years, that paper describes a revised MITG design and fabrication procedure, presents power and weight predictions for that design, and compares these with similar predictions made in 1981. 


\section{Test Results}

As explained in Reference [3], two tests were carried out, each using the test assembly design depicted in Figure 1.

Figure 1. MITG Test Assembly

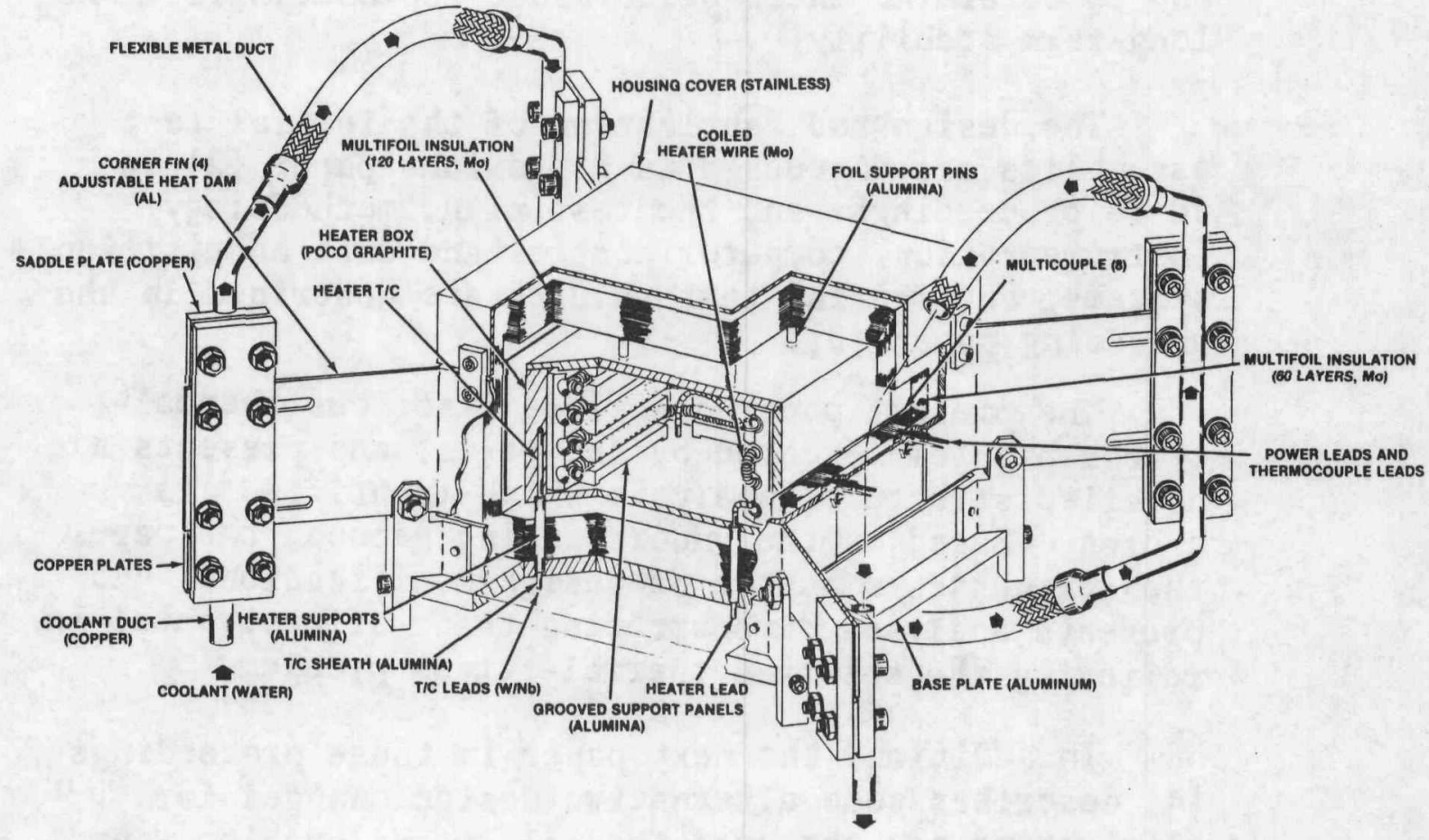

As shown, each test assembly contains eight multicouples, two on each side of the heater box. Each multicouple consists of 18 series-connected thermoelectric couples. Figure 2 shows an exploded view of a typical multicouple used in these tests. Details of the multicouple design and fabrication procedure are described in Reference [2]. 
Figure 2. Exploded View of Multicouple

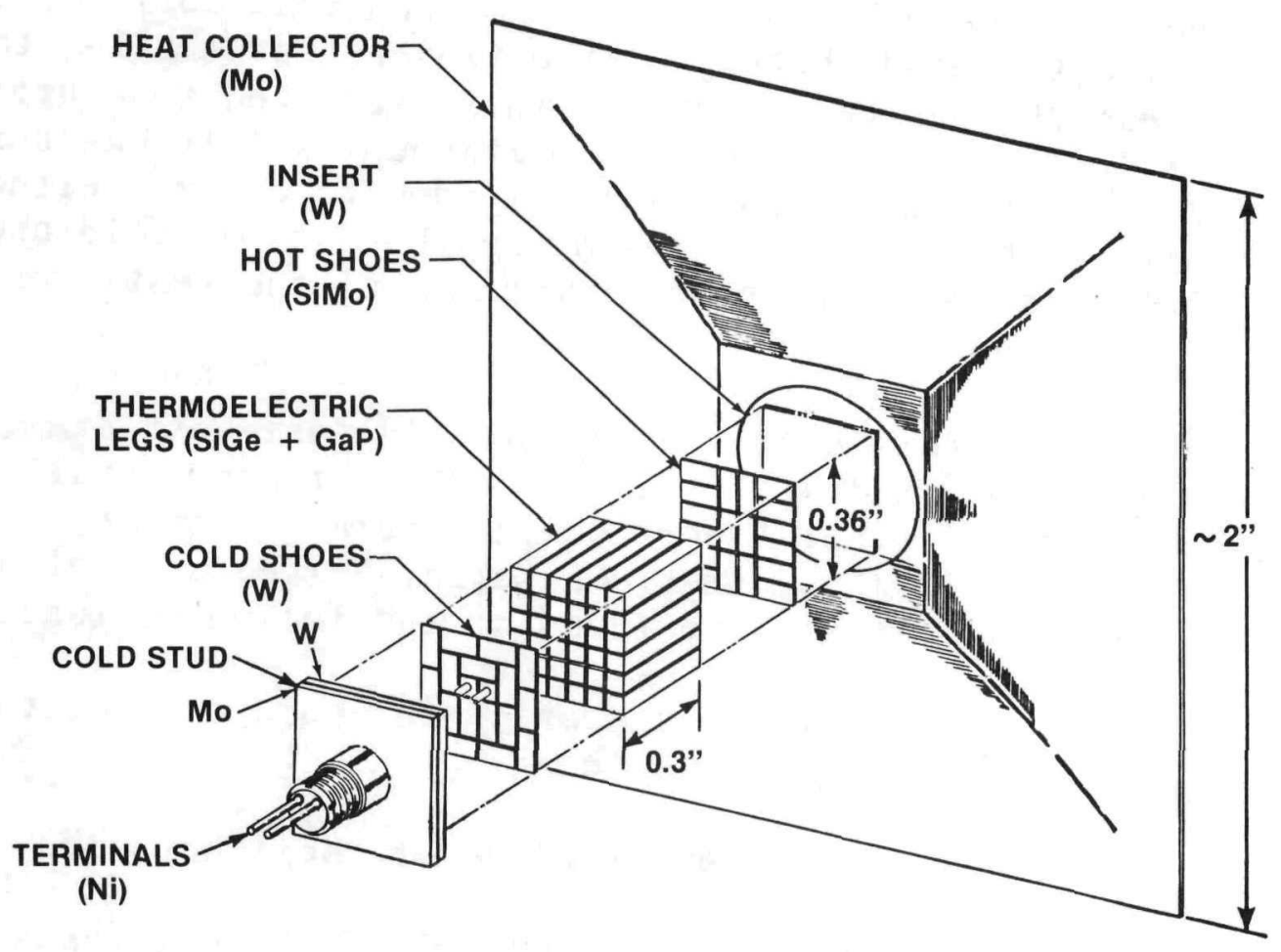

The tests and post-test analyses indicated that the initial multicouple design had a number of problems requiring corrective action. The paper will explain how additional analyses and other studies served to identify the causes of those problems, and to suggest changes in design and fabrication to eliminate them. These changes have been analytically and experimentally evaluated, with very encouraging results. Additional experimental confirmation tests now under way will be described, and their results will be presented at the conference. 


\section{Therma1-Stress Problem}

As reported in Reference [3], a number of electrical and thermal anomalies became apparent during the outgassing and heat-up of the two test assemblies to their design temperatures. These appeared to a greater or lesser degree in all 16 multicouples. It was surmised that these anomalies were due to cracks, either within the leg assemblies or in the hot- or cold-shoes; but this could not be confirmed until the tests were terminated.

After disassembly and detailed post-test examinations conducted at Fairchild and in greater detail at a number of other laboratories (Thermo Electron, Battelle-Columbus, Ames, GE-Valley Forge, and TeledyneTimonium), cracks were found at the following locations:

- Near the center of the cold ends of the leg assemblies

- At the outer edges of their hot ends

- At the outer corners of the cold ends

The first of these was by far the most common and most serious. Such cracks were found in all 16 multicouples, and in many cases had led to complete cleavage at the thermopile's cold end. The cleaved surface was slightly dome-shaped, concave when viewed from the cold end.

As illustrated in Figure 3 , this type of cracking and/or cleavage occurred mostly within the SiGe legs. Thus, it appears that the bonds between the legs and the cold shoes, as well as the glass bonds between the cold shoes and the cold studs, are actually stronger than the SiGe legs themselves. 
Figure 3. Typical Break at Cold End of Thermopile

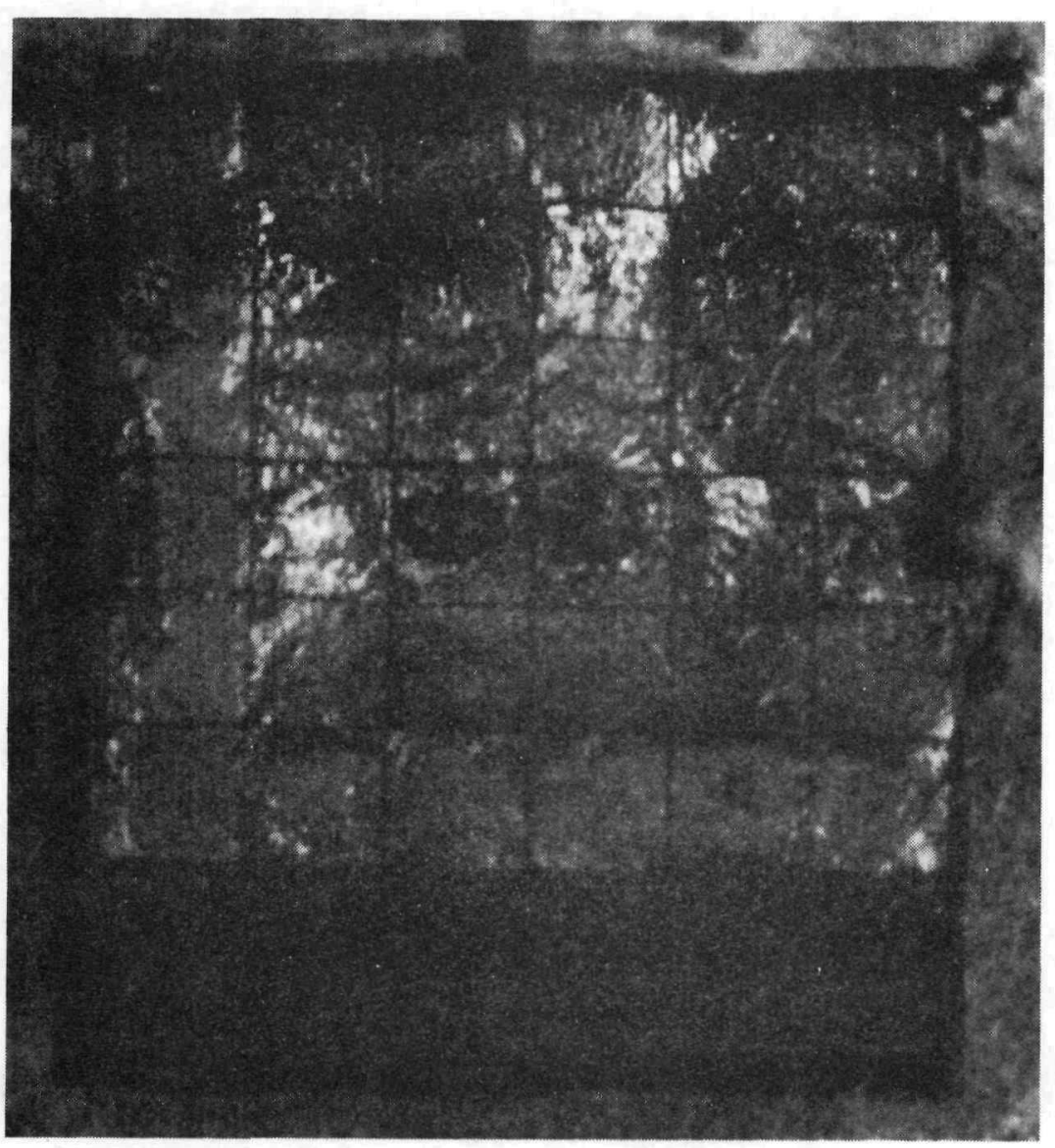


The second type of crack, near the hot ends' outer edges, occurred much less frequently. When they did occur, these were incipient cracks which did not penetrate far toward the center. In no case did they lead to cleavage or loss of the heat collector.

The third type of crack, near the cold ends' outer corners, appeared even less frequently. The only noticeable evidence of their occurrence was occasional discoloration at the corners of the cleavage planes. This suggested that cracking at the corners may have occurred during fabrication or early during outgassing, whereas the cleanliness of the rest of the broken surface indicated that cleavage had occurred much later, at a higher temperature.

The above-described cracking, particularly the first type, is very serious and must be eliminated. It was considered most likely that the observed cracks were produced by thermal stresses resulting from one or more of the following causes:

- Bowing of the bimetallic (W/Mo) cold stud during cooldown and heatup, after fabrication.

- Mismatch in thermal expansion coefficient between the thermoelectric leg assembly and the cold stud and heat collector

- Bowing of the leg assembly's hot and cold faces, due to the large $\left(700^{\circ} \mathrm{C}\right)$ temperature gradient at operating condition.

- The high expansion coefficient of the aluminum wall on which the multicouple is mounted

Each of the above would produce thermal stresses, during fabrication and/or operation, and could be a plausible cause of the observed cracking. Clearly, a detailed stress analysis was needed, covering the thermal history of the multicouple during the various fabrication steps and subsequent heatup to operating temperature. This analysis could identify the dominant cause of thermal stresses. Only then could appropriate design modifications be proposed and evaluated. 


\section{Thermal Stress Analysis}

The model initially used in Fairchild's thermal stress analyses is shown in Figure 4. It represents a $45^{\circ}$ segment, taking advantage of the multicouple's eight-fold symmetry. The model included the tungsten heat collector insert, the glass-bonded SiGe leg assembly, the W/Mo cold stud, the Al housing wall, and a nut (which was assumed to be infinitely stiff). The SiMo hot shoes were not modeled separately, but were incorporated in the SiGe leg assembly. Nor were the thin tungsten cold shoes explicitly represented.

Figure 4. Thermal-Stress Model, Base Case

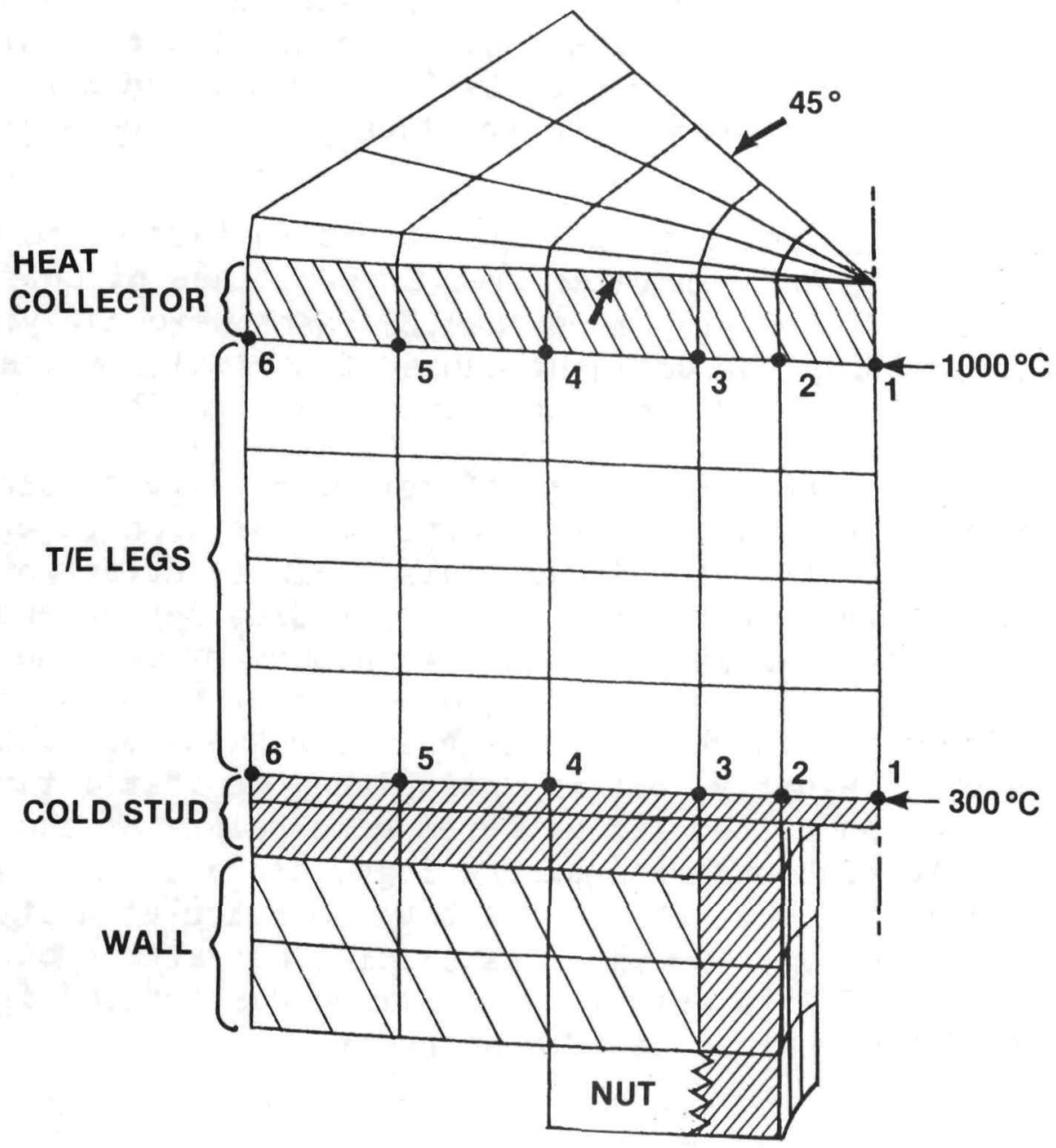


As explained in Reference [5], the analysis made use of the MacNeal-Schwendler version of the NASTRAN computer code, supplemented by the PATRAN pre- and post-processing software of PDA Engineering. A special scheme for accommodating temperature-dependent expansion coefficients was developed by Fairchild. The NASTRAN model contained 144 solid-shell elements connecting 1290 grid points, for a total of 3000 static degrees of freedom. Both 20-node hexahedral elements and 15-node. pentahedral elements were used.

Each case analyzed consisted of seven to nine subcases. As explained in [5], these subcases were needed to simulate the thermal cycles involved in brazing the bimetallic cold stud at $980^{\circ} \mathrm{C}$, making a high-temperature $\left(1100^{\circ} \mathrm{C}\right) \mathrm{glass}$ bond between the thermopile and the heat collector, making a low-temperature $\left(680^{\circ} \mathrm{C}\right)$ glass bond between the thermopile and the cold stud, bolting the multicouple to the aluminum housing, and heating the assembly to its $1000^{\circ} \mathrm{C} / 300^{\circ} \mathrm{C}$ operating temperature.

A typical case required three to four hours of CPU time, and generated over 80,000 lines of output data. Ultimately, more than 25 cases were analyzed to identify the dominant cause of thermal stresses and to evaluate a variety of possible design fixes.

To reduce the flood of output data to tractable form, a post-processing program was written to extract only the most significant data. First, attention was restricted to stresses in the SiGe leg assembly, since that is where the cracks had occurred. Next, attention was confined to the diagonal boundary plane, since that is where the highest stresses were found to occur. Finally, within that plane, we restricted our attention to the six numbered mesh points at the upper and lower boundaries of the legs, since that is where cracks had been observed and where calculated stresses were highest. By thus restricting our attention, the number of data sets to be examined was reduced from 1290 mesh points to 12 mesh points. 
The computed results for those 12 mesh point are displayed in Table 1, which presents both the fabrication stresses, after cooldown, and the operating stresses with the $1000^{\circ} \mathrm{C} / 300^{\circ} \mathrm{C}$ temperature gradient.

The data presented are for the base case; i.e., the case representing the multicouple design in the initial test assemblies. Note that all stresses have been rounded off to the nearest ksi. Although this often leaves only one significant figure, the resultant clarity makes it much easier to discern trends and differences between alternative designs.

Table 1. Base Case

Tensile Stresses in SiGe, in ksi

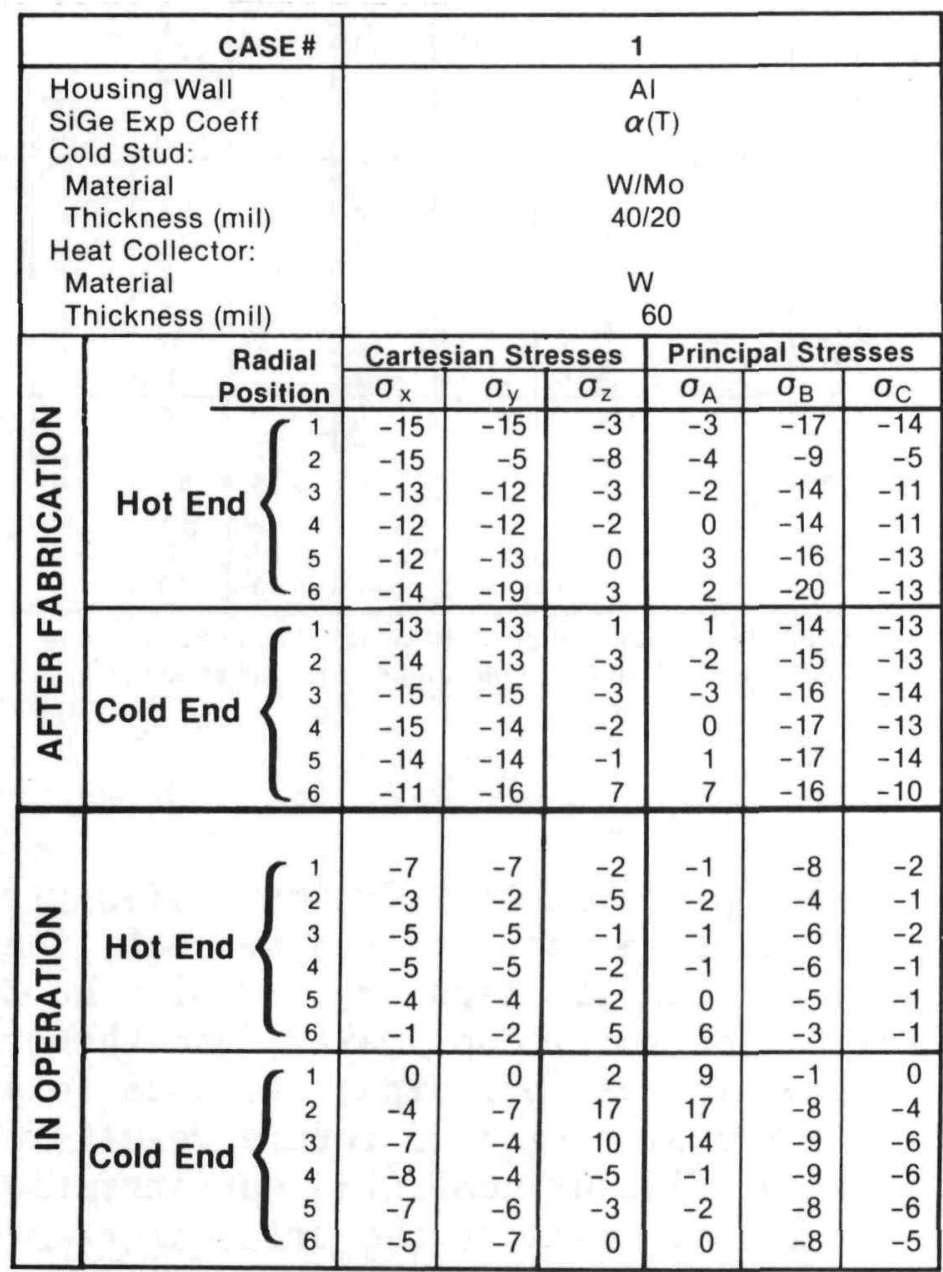

- This demonstrates that $\sigma_{2}$ is the dominant stress, and is essentially identical to the largest principal stress $\left(\sigma_{A}\right)$ 
Table 1. Base Case

Tensile Stresses in SiGe, in ksi

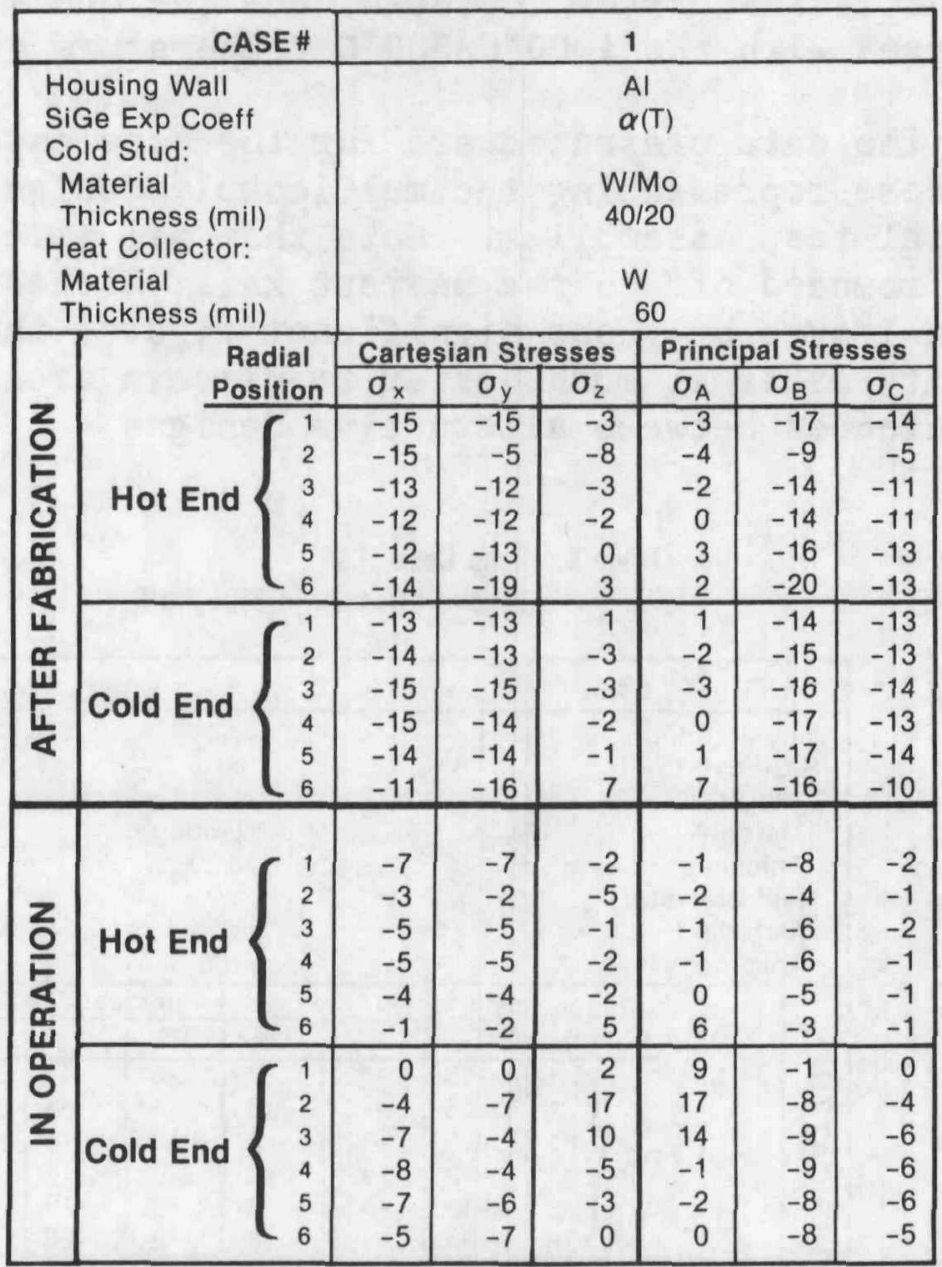

- This demonstrates that $\sigma_{\mathrm{z}}$ is the dominant stress, and is essentially identical to the largest principal stress $\left(\sigma_{A}\right)$

Table 1 presents both the cartesian stresses and the principal stresses. As can be seen, the highest cartesian tensile stresses $\left(\sigma_{7}\right)$ are in the $Z$-direction, parallel to the multicouple axis; and these are very close to the largest principal stresses $\left(\sigma_{A}\right)$. Consequently, our examination of future results can be further simplified by confining our attention to the axial tensile stresses at the critical 12 mesh points. This is illustrated in Table 2, for the base case. 
Table 2. Base Case

Axial Tensile Stresses in SiGe, in ksi

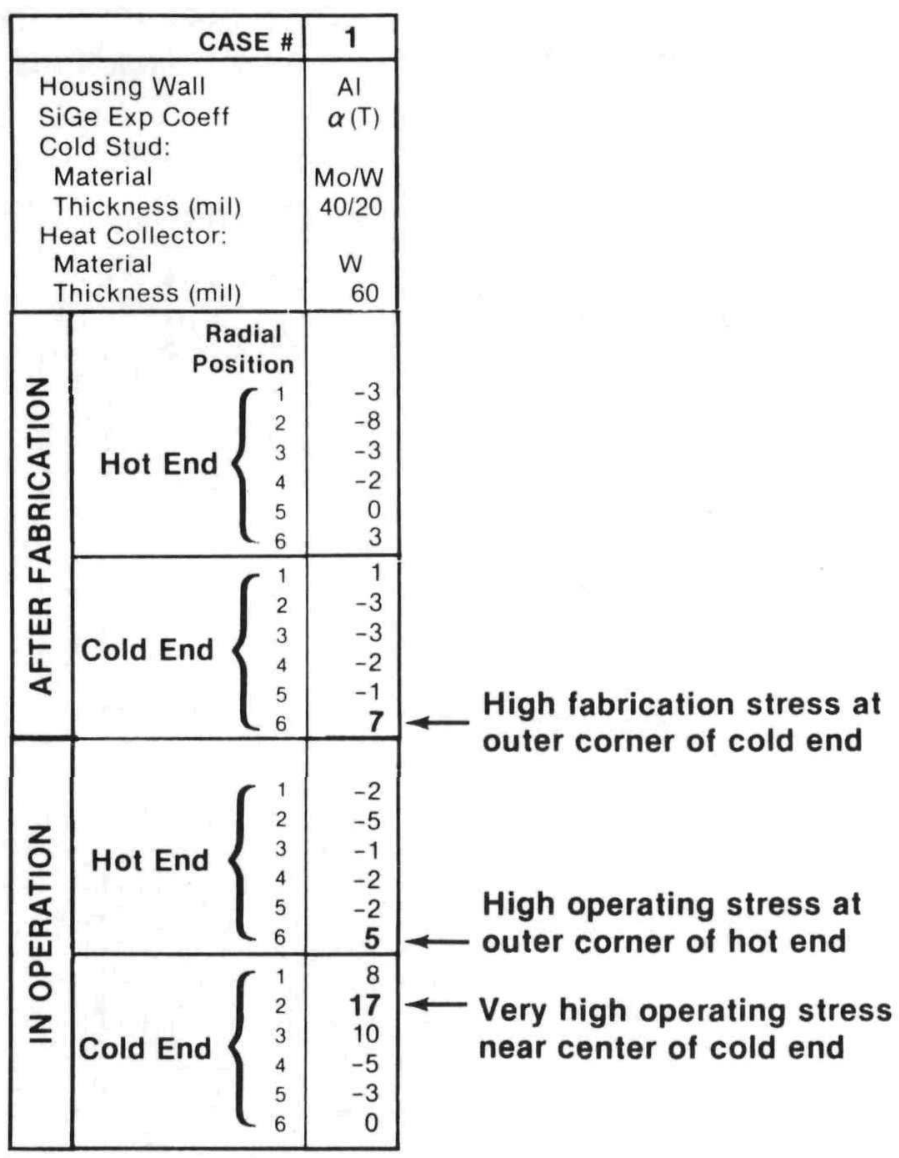

The most interesting thing about Table 2 is how closely the analytical results match the experimental findings. The worst stress is the $17 \mathrm{ksi}$ operating stress near the center of the leg assembly's cold end, precisely where the dome-shaped cleavages had occurred. The operating stress at the outer corner of the hot end and the fabrication stress at the corner of the cold end are lower, but still too high. These are the locations where secondary damage was observed in the tests. This excellent agreement between the experimental and analytical results lends confidence in the validity of the analytical model and methodology. 
The analytical results for the base case are displayed graphically in Figure 5. This, and similar figures presented later, shows (in exaggerated form) the deflections due to the eperational temperature gradients. Note the curvature of the upper and lower faces of the SiGe assembly, as predicted by theory, and also the thermal expansion of the aluminum wall.

Figure 5. Base Case Deformation

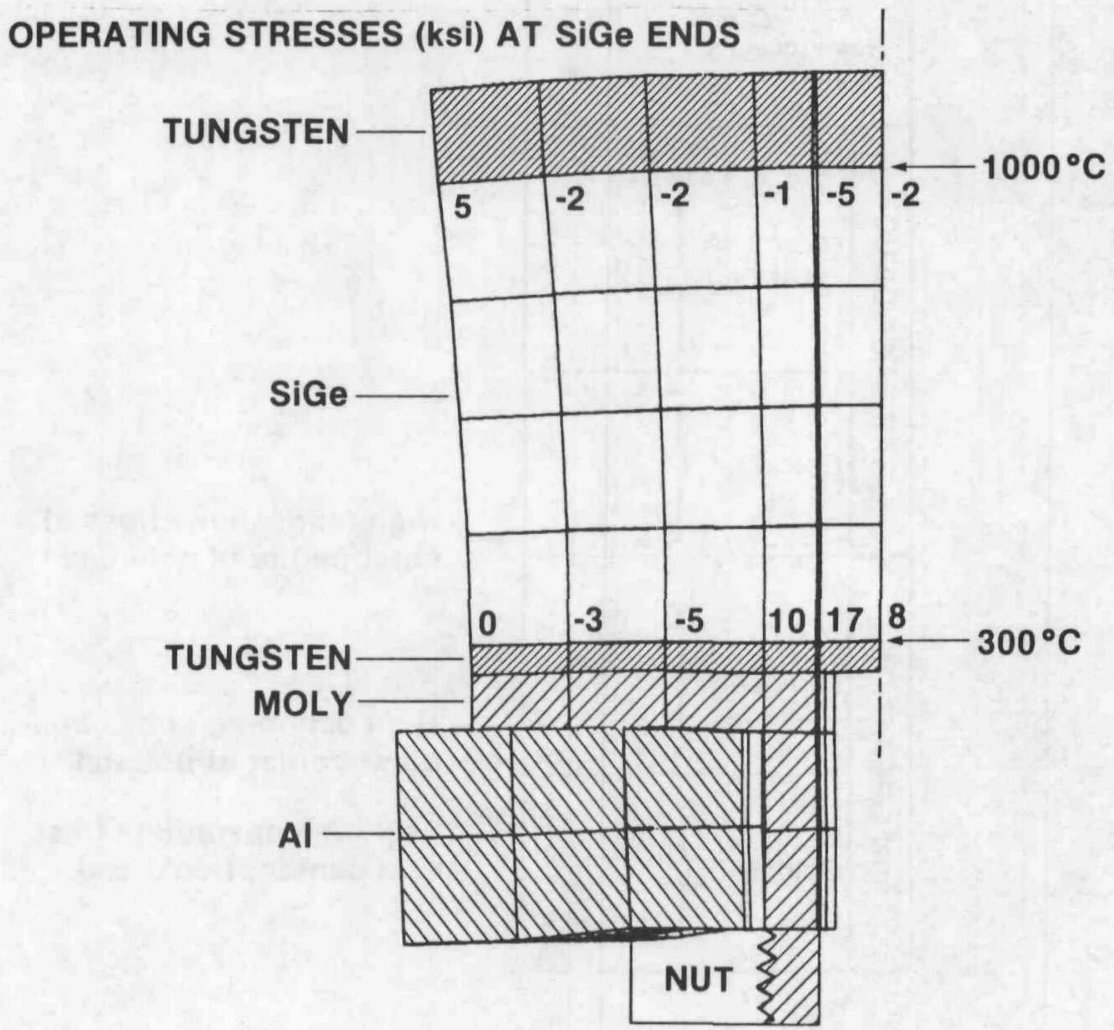

The figure also shows the axial stresses at the 12 critical points under operating conditions (in ksi). The latter are obtained by superposition of the fabrication and temperature-gradient stresses.

\section{Dominant Source of Thermal Stresses}

Having gained confidence in the validity of the analysis, the model was next used to identify the dominant source(s) of the thermal stresses, and to evaluate design modifications to eliminate or substantially reduce those stresses. The four possible causes of thermal stress listed at the end of Section 3 were investigated one at a time. 


\subsection{Bimetallic Cold Stud}

First, we analyzed a case identical to the base case except for the use of an all-tungsten cold stud. This was to eliminate the effect of the bimetallic cold stud, which would tend to change its curvature when it was heated or cooled. The results of that change are presented in Table 3 (Case 3 ).

As shown, elimination of the bimetallic cold stud reduces the fabrication stress at the cold end's corners from $8 \mathrm{ksi}$ to $1 \mathrm{ksi}$. But it does nothing to reduce the worst problem, the very high operating stress near the center of the cold end. In fact, that stress actually went up a little, probably because of the higher modulus of tungsten.

Table 3. Effect of Bimetallic Cold Stud

AXIAL TENSILE STRESSES IN SiGe, IN ksi

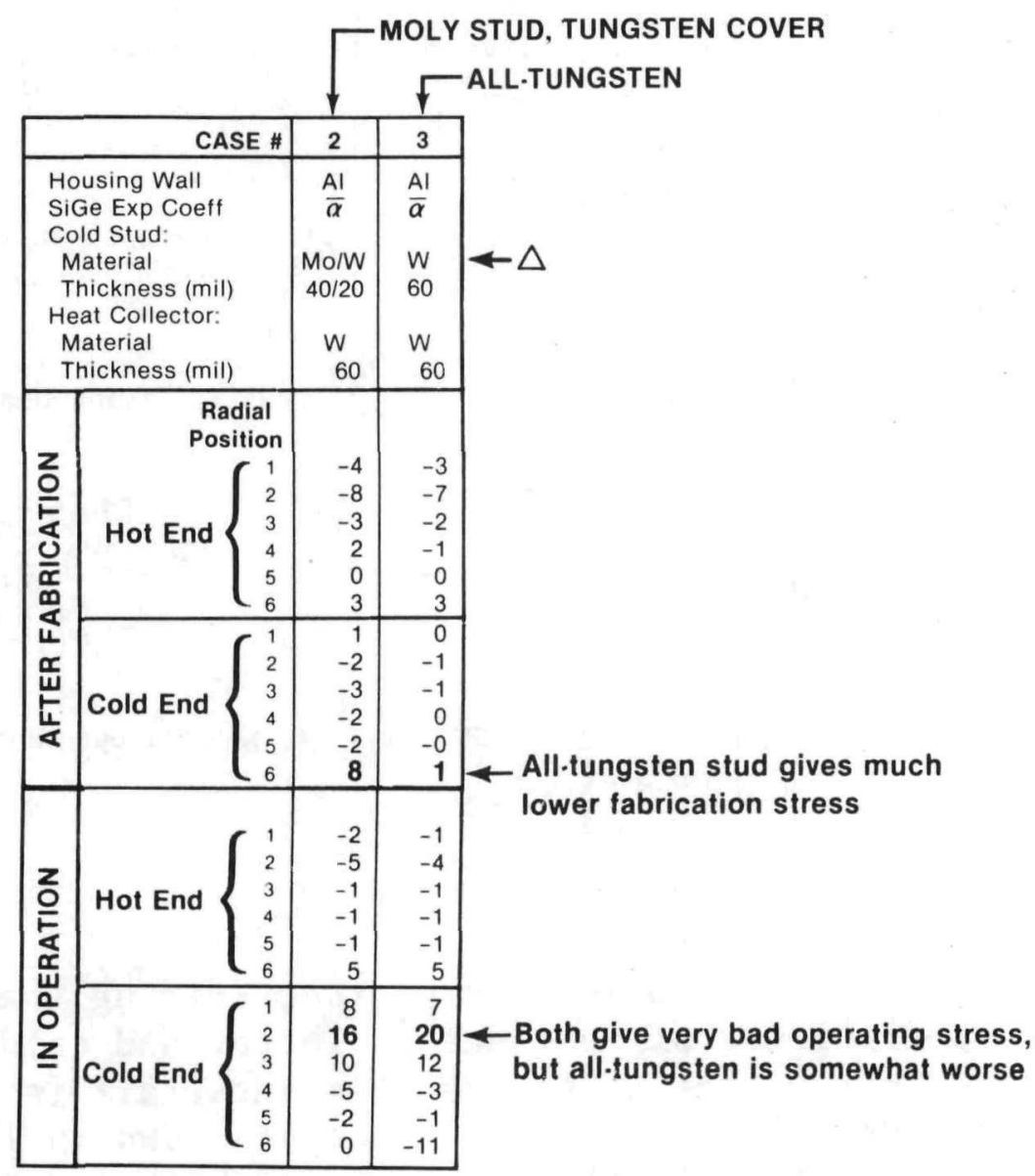




\subsection{Mismatched Expansion Coefficients}

The second possible cause investigated was the difference in expansion coefficients between SiGe and tungsten. Although these two materials are very close, tungsten does have a slightly higher coefficient. To eliminate this effect, a case was run in which the expansion coefficient of tungsten was arbitrarily set equal to that of SiGe. The resultant stresses are shown in Table 4 (Case 4).

Table 4. Effect of Matched Expansion Coefficients

AXIAL TENSILE STRESSES IN SiGe, IN ksi

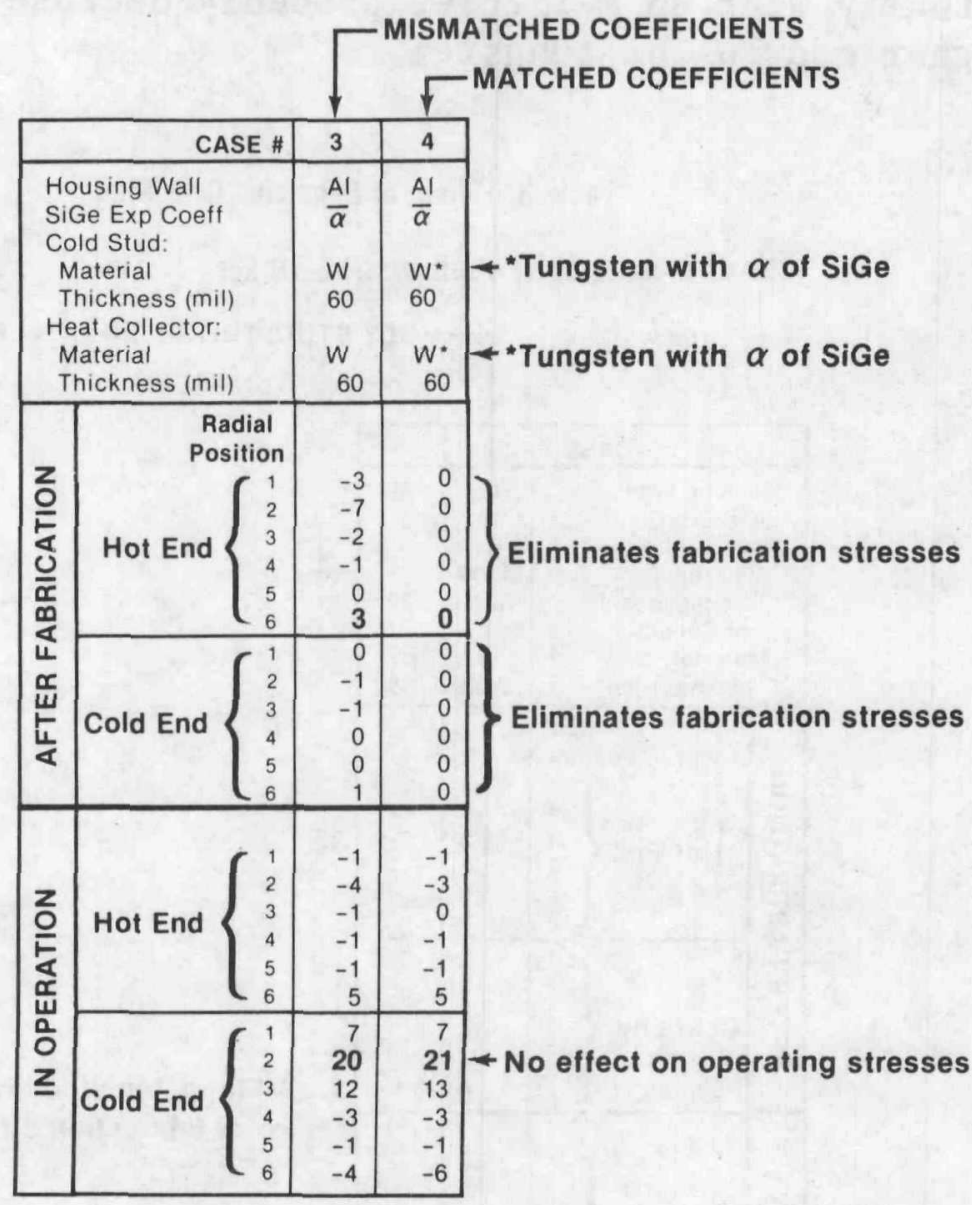

As would be expected; setting the expansion coefficients of the heat collector and cold stud equal to that of the SiGe legs eliminates all fabrication stresses, at both ends. But, as shown in Table 4, it has no significant effect on the high operating stresses. 


\subsection{Effect of Temperature Gradient}

Having eliminated the bimetallic cold stud and the difference in expansion coefficients as the dominant cause of the high operating stresses at the cold end of the multicouple, we were left with two other possible explanations: the curvature of the $\mathrm{SiGe}$ leg assembly as the result of the $700^{\circ} \mathrm{C}$ temperature gradient; and the high expansion coefficient of the aluminum wall.

The first of these effects is illustrated in Figure 6 , which shows what the stress-free deformation of the unbonded components would be at their operating temperatures. As can be seen, the $\mathrm{SiGe}$ assembly would assume a convex curvature at its upper (hot) surface and a concave curvature at its lower (cold) surface. The corresponding tungsten parts would, of course, remain flat.

Figure 6. Effect of Temperature Gradient on Unbonded Parts

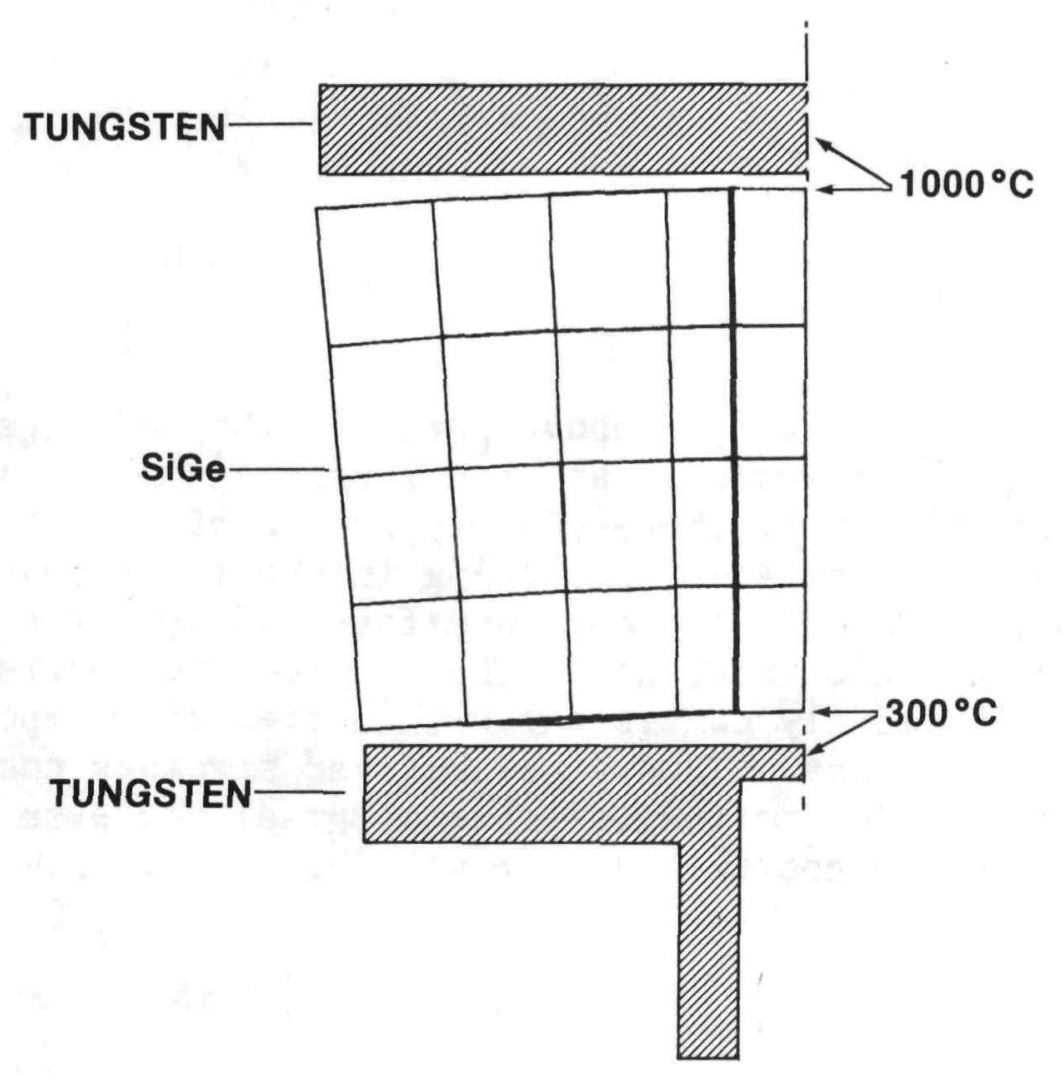


Figure 6. Effect of Temperature Gradient on Unbonded Parts

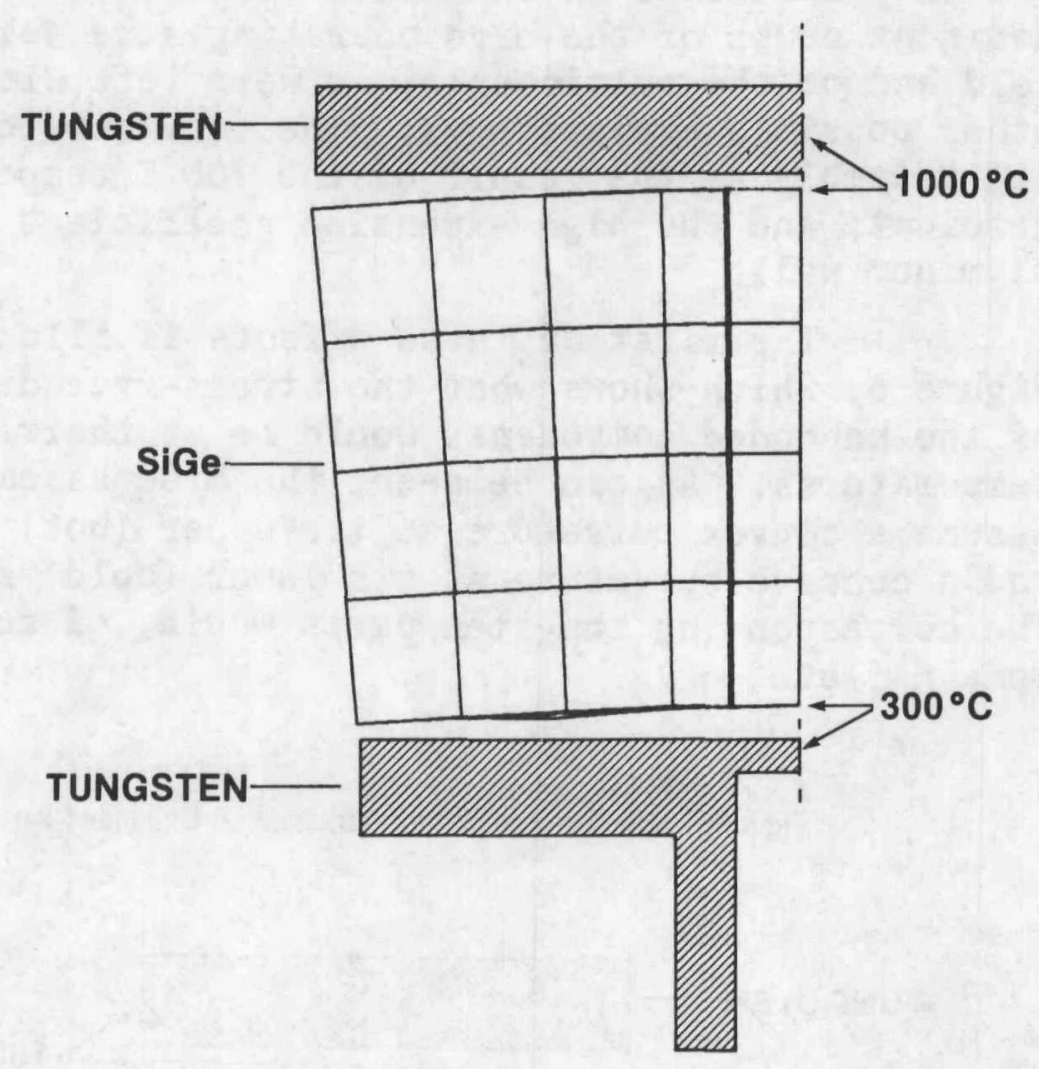

When the above parts are bonded together, the respective surfaces at each interface must remain congruent. Thus, there clearly is a conflict at each of the interfaces, resulting in tensile stresses near the outside of the hot interface and near the center of the cold interface. These theoretical stresses are qualitatively consistent with our earlier experimental observations. Thus, the observed stresses could in fact be due to the temperature gradient, even if the expansion coefficients of all the materials were identical. 
To test whether this was the case, or whether the dominant stress source was the high expansion coefficient of the aluminum wall, the base case multicouple was analyzed without any wall. The results of that analysis are displayed in Figure 7 and in Table 5.

Figure 7. Effect of Omitting Aluminum Wall from Base Case

OPERATING STRESSES (ksi) AT SiGe ENDS

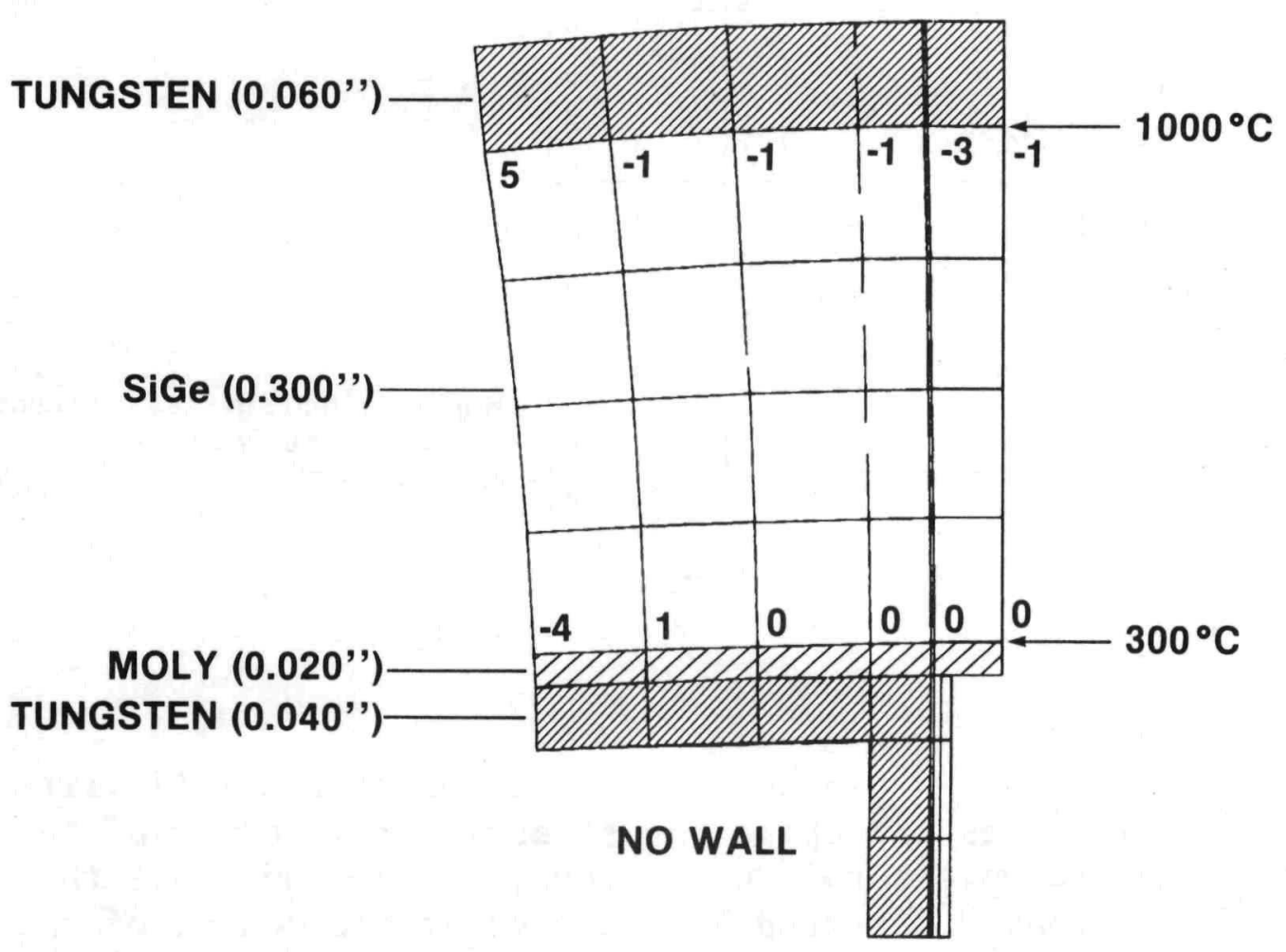


Table 5. Effect of Aluminum Wall

Axial Tensile Stresses in SiGe, in ksi

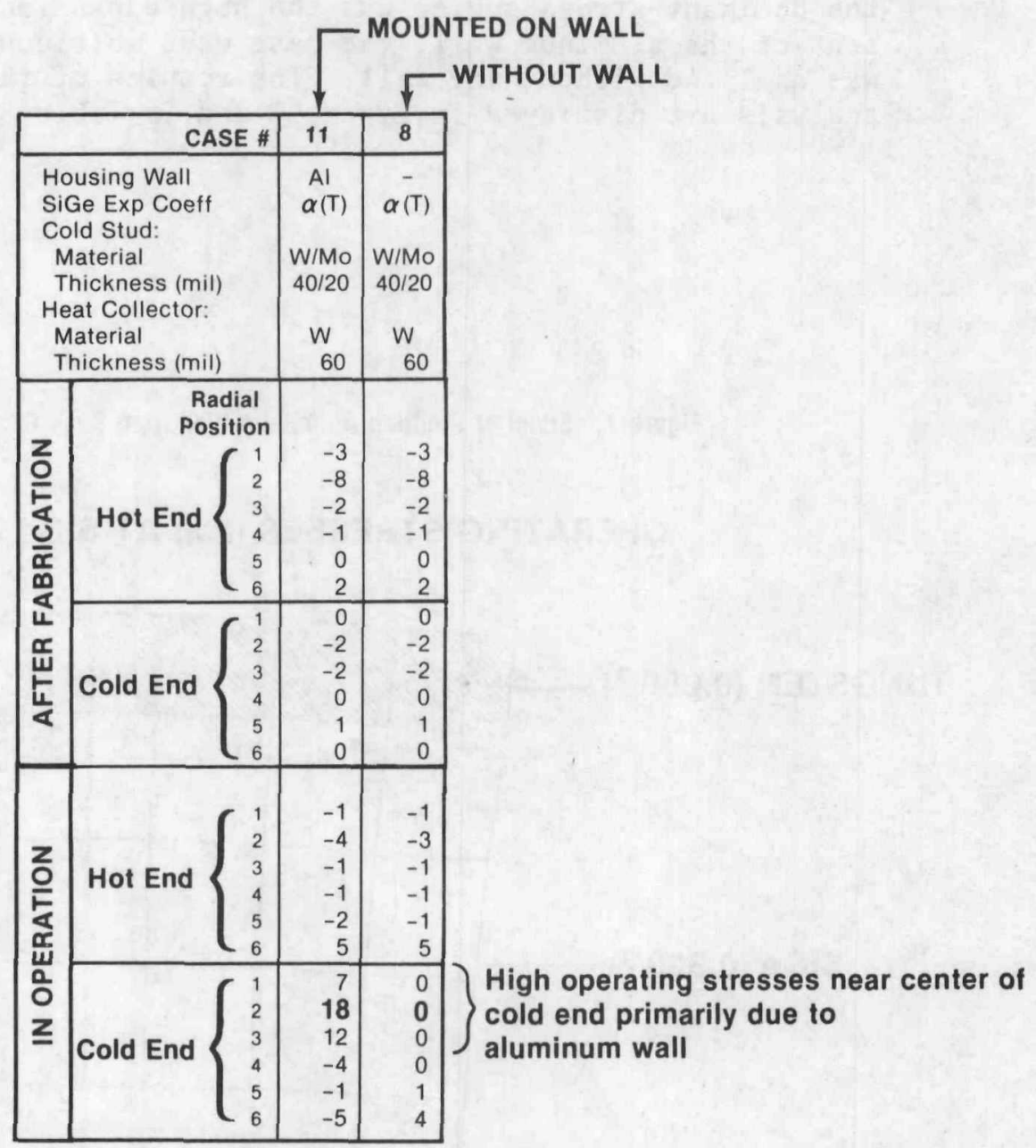

As can be seen, omission of the wall virtually eliminates the operating stress at the center of the multicouple's cold end. Thus, we conclude that the previously observed high stress at the eenter of the cold end was not due to the curvature of the leg assembly shown in Figure 6. Rather, it was due to the high expansion coefficient of the aluminum wall. 


\subsection{Wall Expansion Effect}

To confirm this conclusion, we analyzed a case identical to the base case, except that the multicouple was bolted to a molybdenum wall, which matched the expansion coefficient of the cold stud. The results of that analysis are shown in Figure 8 .

Figure 8. Base Case with Molybdenum Wall

\section{OPERATING STRESSES (ksi) AT SiGe ENDS}

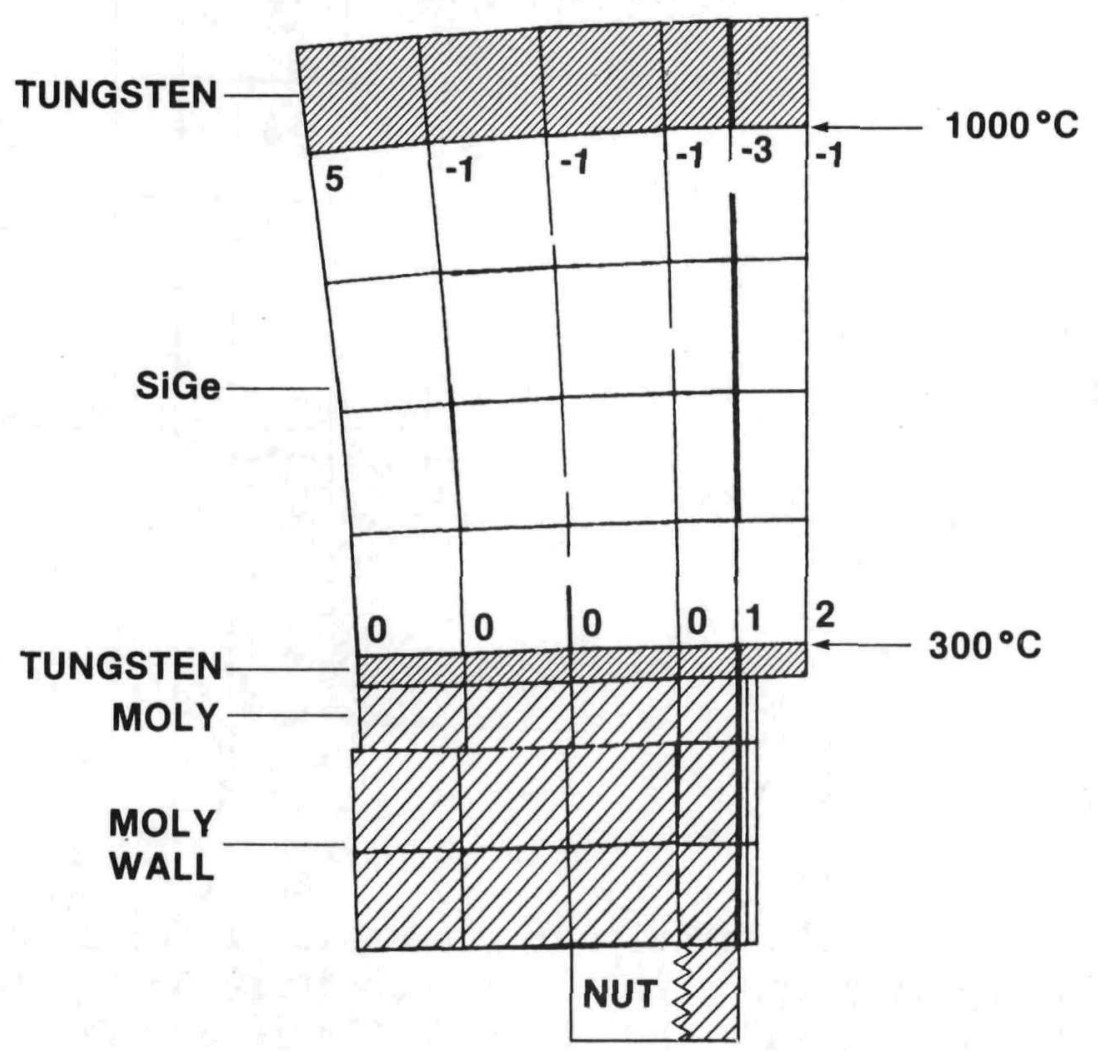

As can be seen, the high operating stresses near the center of the cold end virtually yanish when the expansion coefficient of the wall matches that of the cold stud.

These results confirm that the dominant stress source is the high expansion of the aluminum wall. 
The mechanism for this is clear from inspection of Figure 5. Near the axis of the multicouple, the expansion of the aluminum wall is resisted by the tension in the stud pin. But near the outside, the expansion of the aluminum causes the edges of the stud head to curve upward.

Figure 5. Base Case Deformation

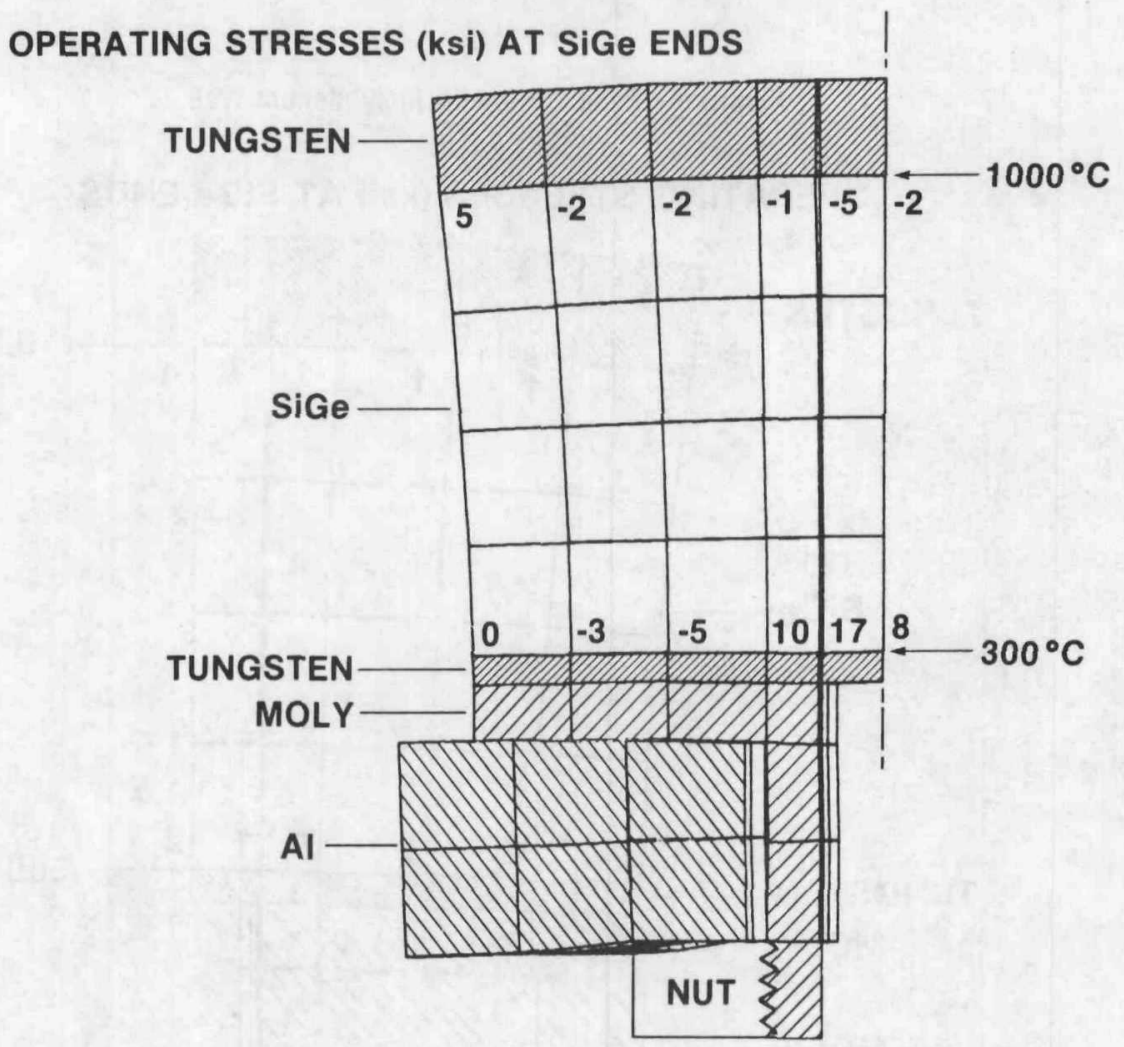

The resultant curvature of the stud surface is exactly opposite to the curvature of the leg assembly's cold end shown in Figure 6 . Thus, the expansion of the aluminum wall causes a high operating stress at the center of the cold end, which led to the very serious cracks and cleavages observed in the initial tests.

It may legitimately be asked why the above analyses were not undertaken before the test hardware was built. In retrospect, they certainly should have been. But these analyses represent a major and time-consuming effort, and there was no a-priori evidence that they were needed, or that they would prove as informative and productive as they did. 


\section{Elimination of Thermal Stress}

The principal cause of the thermal stresses having been determined, a number of possible design changes to eliminate those stresses were investigated. Some of these are discussed below:

\subsection{Compliant Cushion}

One possible solution would be to insert a low-modulus cushion between the cold stud and the SiGe assembly, to decouple the latter from the aluminum expansion effect. To this end, Thermo Electron based on prior experience on another program - had recommended the addition of a thin graphite layer (Stackpole 2128) between the tungsten cold stud and the SiGe assembly. These two materials have very similar expansion coefficients, have been successfully. bonded to each other, and the graphite has an attractively low modulus ( $10^{6} \mathrm{psi}$ ). When this suggestion was analyzed by Fairchild, it yielded the results shown in Figure 9 and Table 6.

Figure 9. Effect of Graphite-Cushioned Cold Stud

OPERATING STRESSES (ksi) AT SiGe ENDS

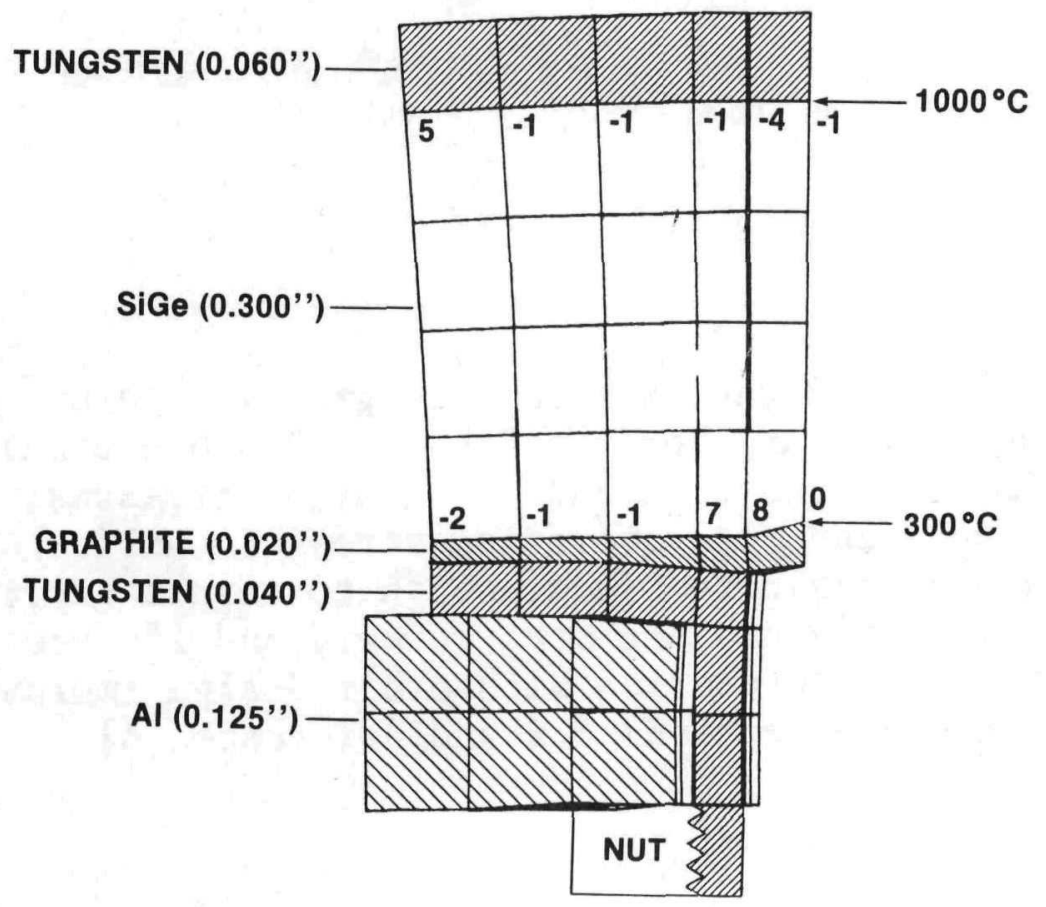


Table 6. Effect of Graphite in Cold Stud

Axial Tensile Stresses in SiGe, in ksi

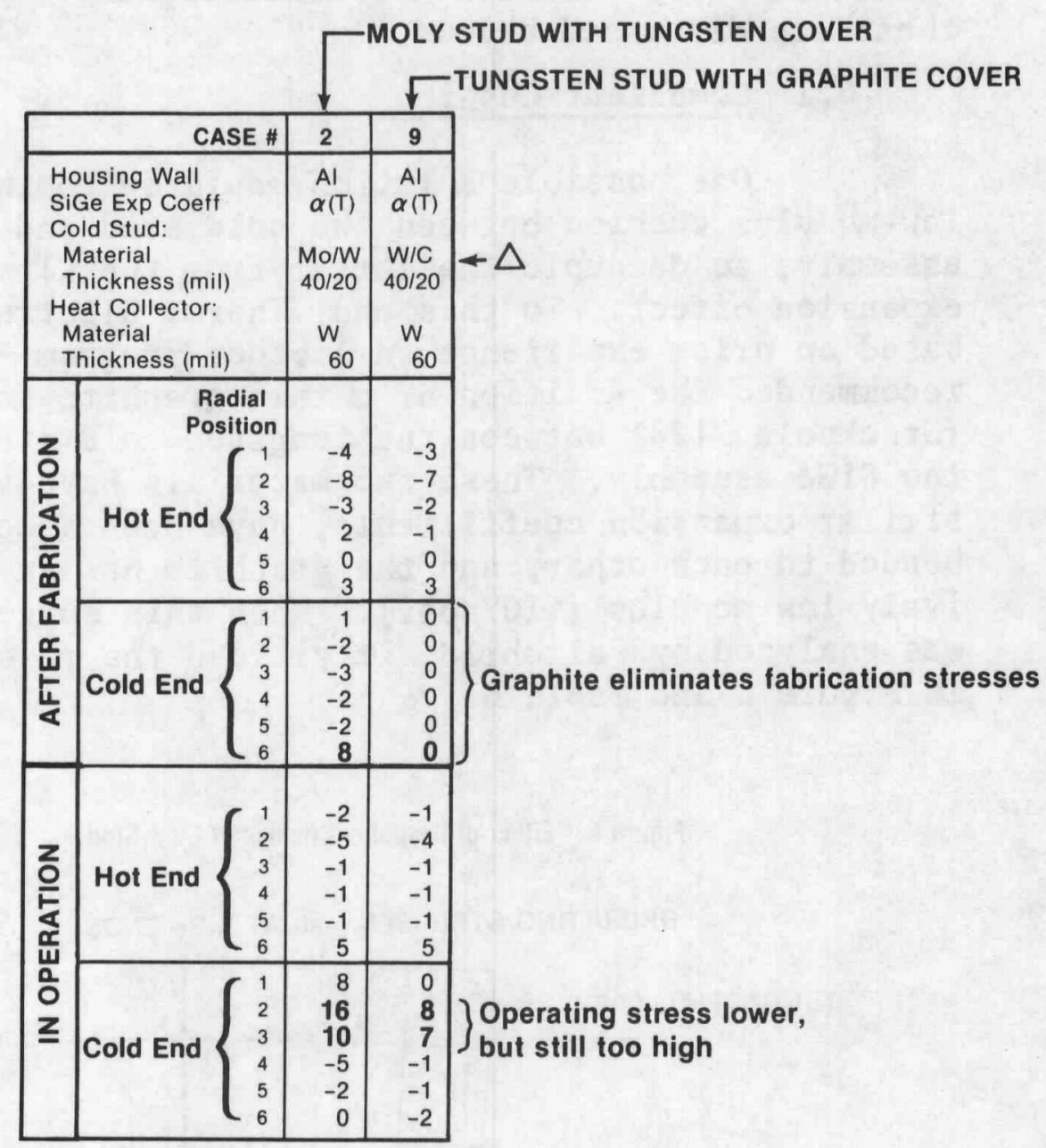

As can be seen, the graphite layer is quite compliant. As shown in Table 6, its use completely eliminates the cold-end fabrication stresses, and cuts the cold-end peak operating stress in half. While this was not considered good enough to ensure survival by itself, it was a big step forward and its use is beneficial in conjunction with other design improvements, as shown in Sections 6.2 and Reference [4]. 
In addition to the graphite-cushioned cold stud, Thermo Electron had also suggested the use of a graphite heat collector, in place of the bimetallic (Mo/W) structure used in the initial test assemblies (see Figure 2). This suggestion was analyzed by Fairchild, with the results shown in Table 7.

Table 7. Effect of Equal-Thickness of Graphite Heat Collector

AXIAL TENSILE STRESSES IN SiGe, IN ksi

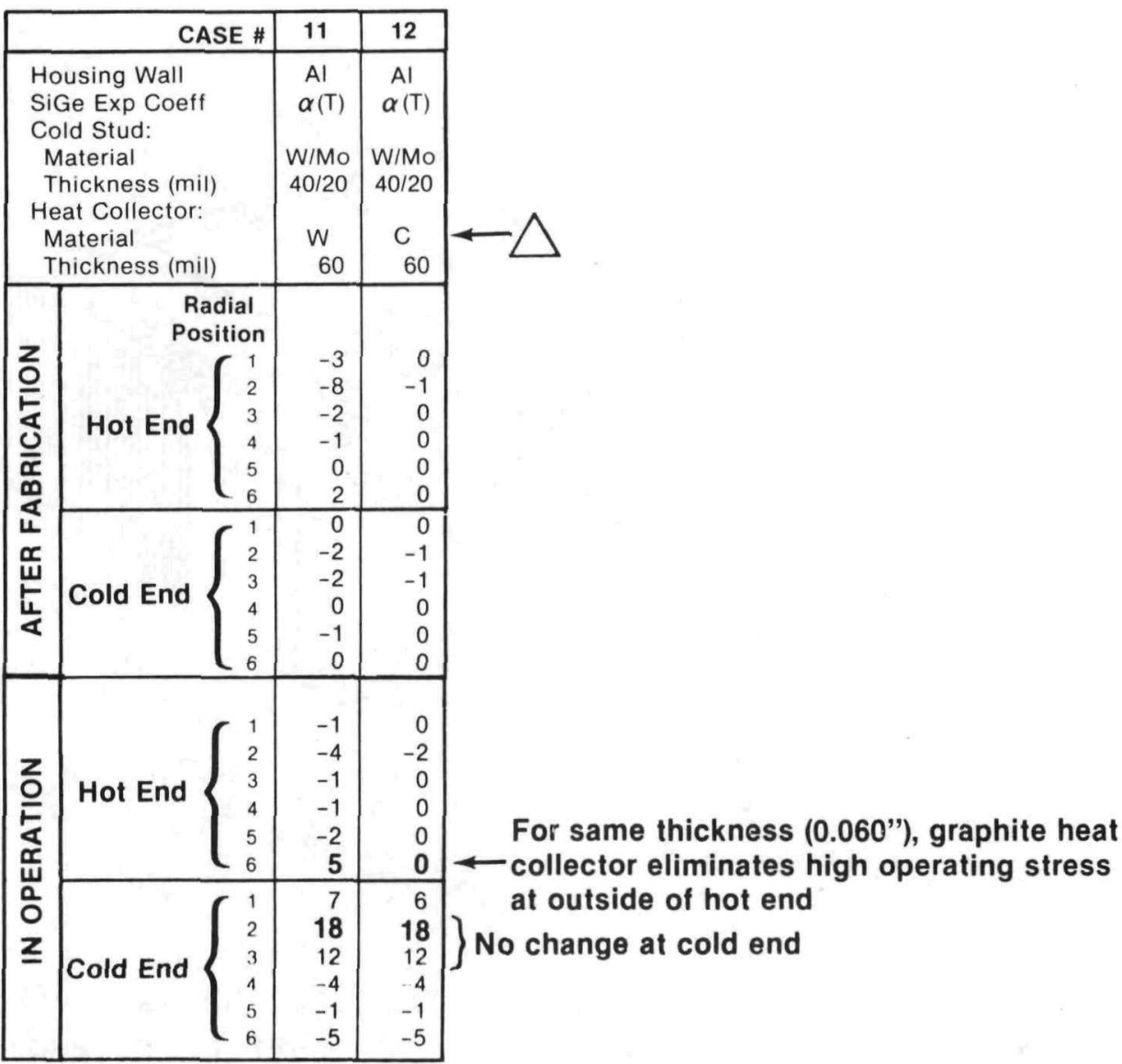

As can be seen, the operating tensile stresses at the multicouple's hot end would be completely eliminated by the use of a graphite heat collector of equal thickness as before. However, this conclusion is somewhat misleading, because in reality a graphite heat collector must be almost three times as thick, as explained in Section 6.3. 


\subsection{Double-Headed Cold Stud}

Another design change, suggested by Fairchild, was the use of a double-headed cold stud, illustrated in Figure 10.

Figure 10. Double-Headed Cold Stud Design

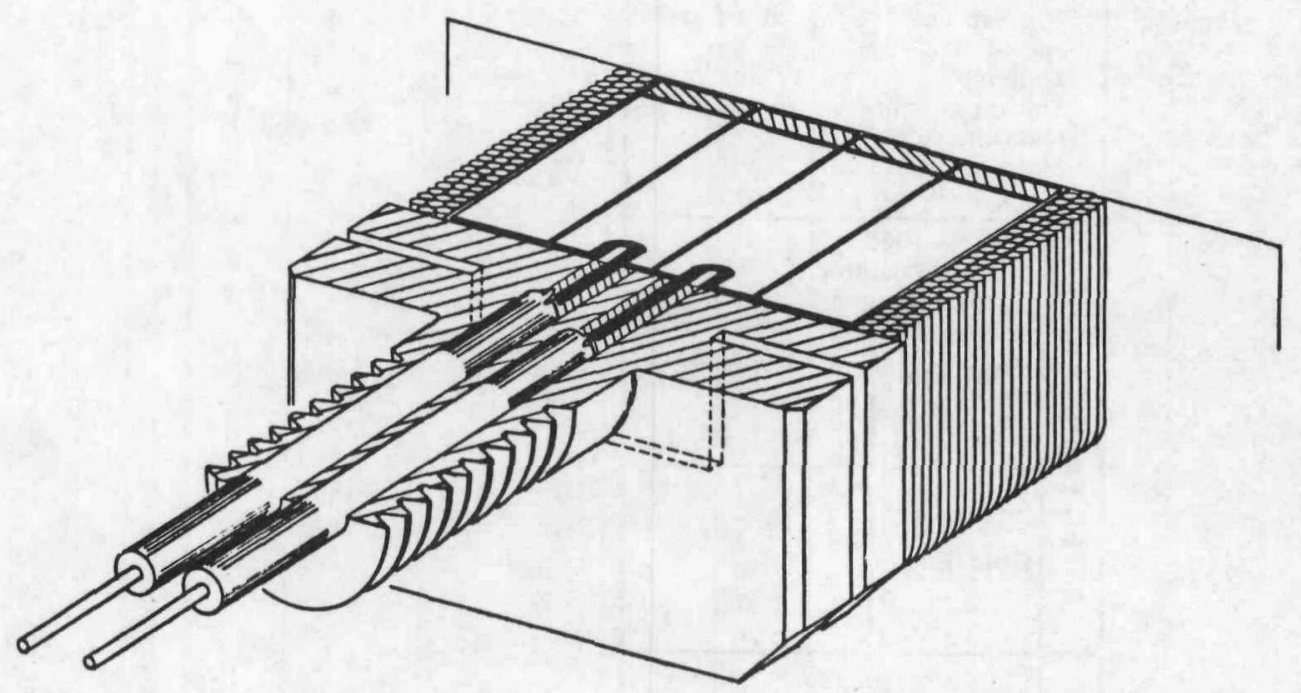

As shown, one of the stud heads is bonded to the SiGe thermopile, while the other bears against the aluminum housing wall. The intervening stress-relief slots (from all four edges) effectively serve to isolate the thermopile from the effects of the aluminum wall expansion. 
However, the slots shown in Figure 10 impede not only stress transmission but also heat transmission. This is illustrated in Figure 11, which shows the temperature distribution for a typical design.

Figure 11. Temperature Map (in ${ }^{\circ} \mathrm{C}$ ) of Double-Headed Cold Stud

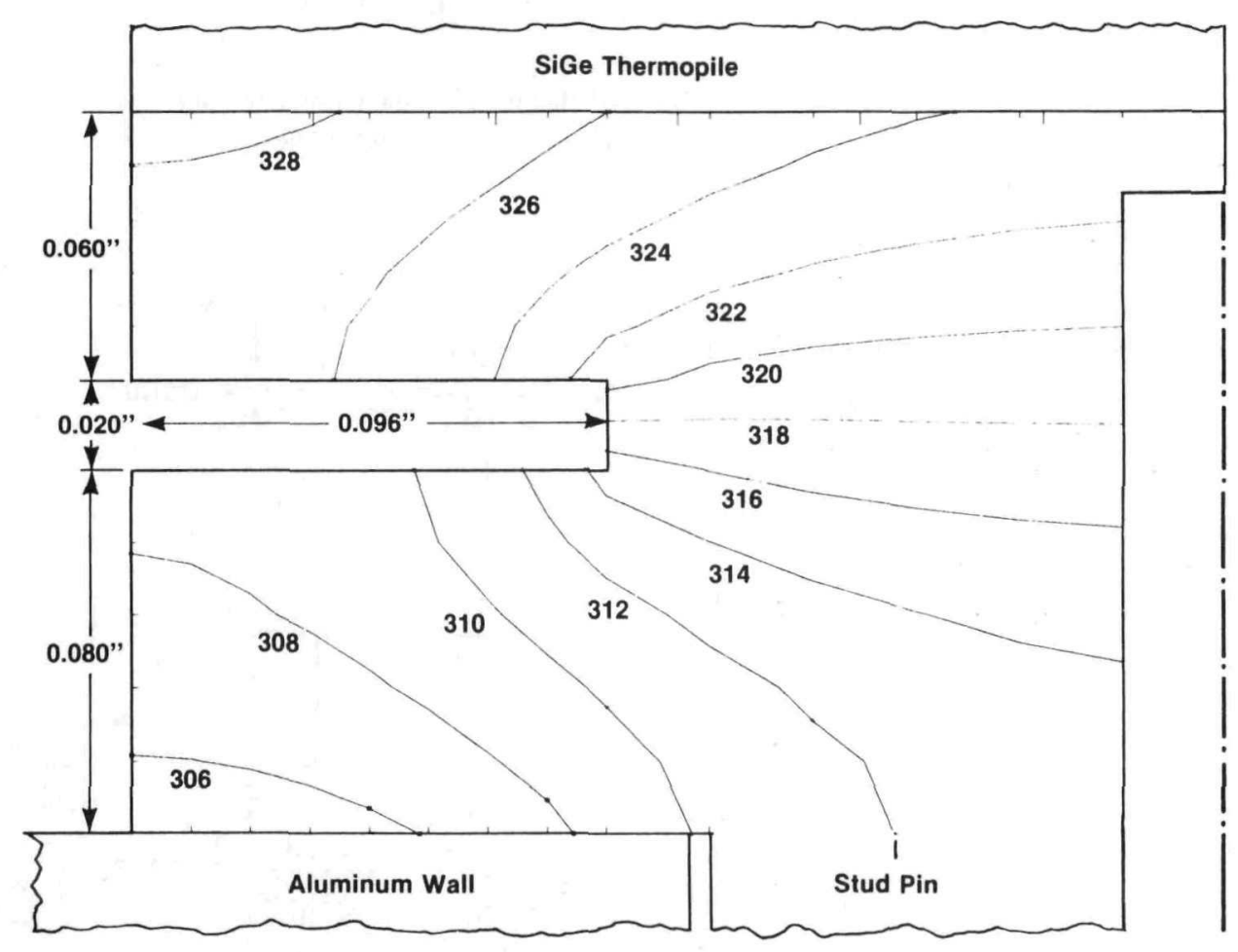

As can be seen, the temperature drops for the dimensions shown in Figure 11 are considerable but not prohibitive. Therefore, these dimensions were tentatively selected for the stress analysis required to evaluate the merit of the concept. 
Again, symmetry conditions were invoked to limit the stress-analysis model to a $45^{\circ}$ segment. The computed results for a $0.060^{\prime \prime}$ heat collector and an all-tungsten double-headed cold stud are presented in Figure 12 and Table 8.

The effectiveness of the stress relief slots in isolating the SiGe legs from the aluminum expansion effects is clearly shown by the deflection plots. As can be seen, the edges of the lower stud-head curve upward, as the result of the aluminum expansion; while those of the upper stud-head curve downward, as the result of the SiGe temperature gradient.

Figure 12. Effect of Double-Headed Tungsten Stud Operating Stresses at SiGe Ends in ksi

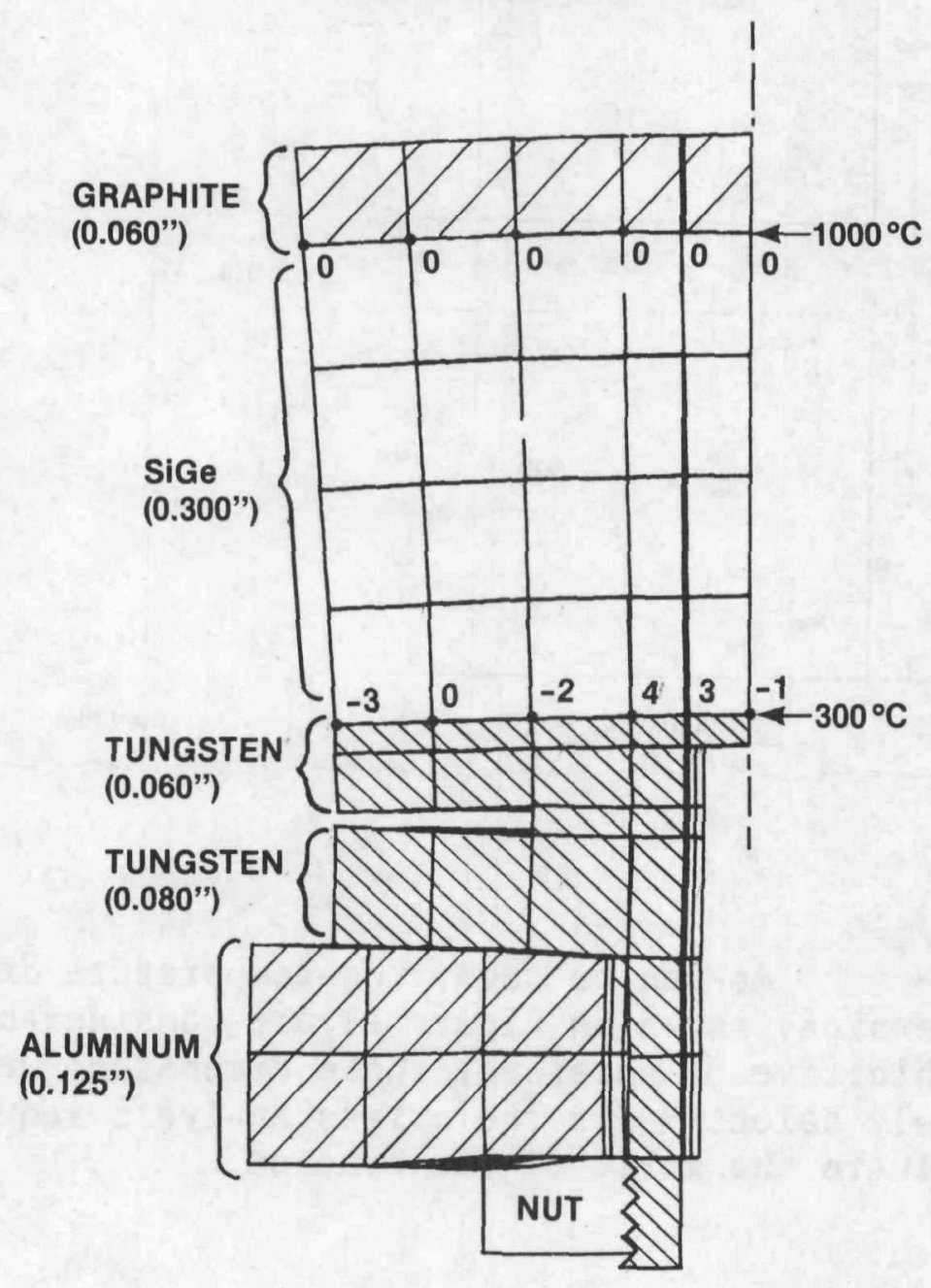


Table 8 compares the cold-end stresses for single-headed and double-headed cold studs. As can be seen, the double-headed stud has slightly higher fabrication stresses, as the result of its greater thickness and stiffness. But its peak operating stress is only one fifth that of the single-headed stud.

Table 8. Comparison of Single-Headed and Double-Headed Tungsten Studs

AXIAL TENSILE STRESSES IN SiGe, IN ksi

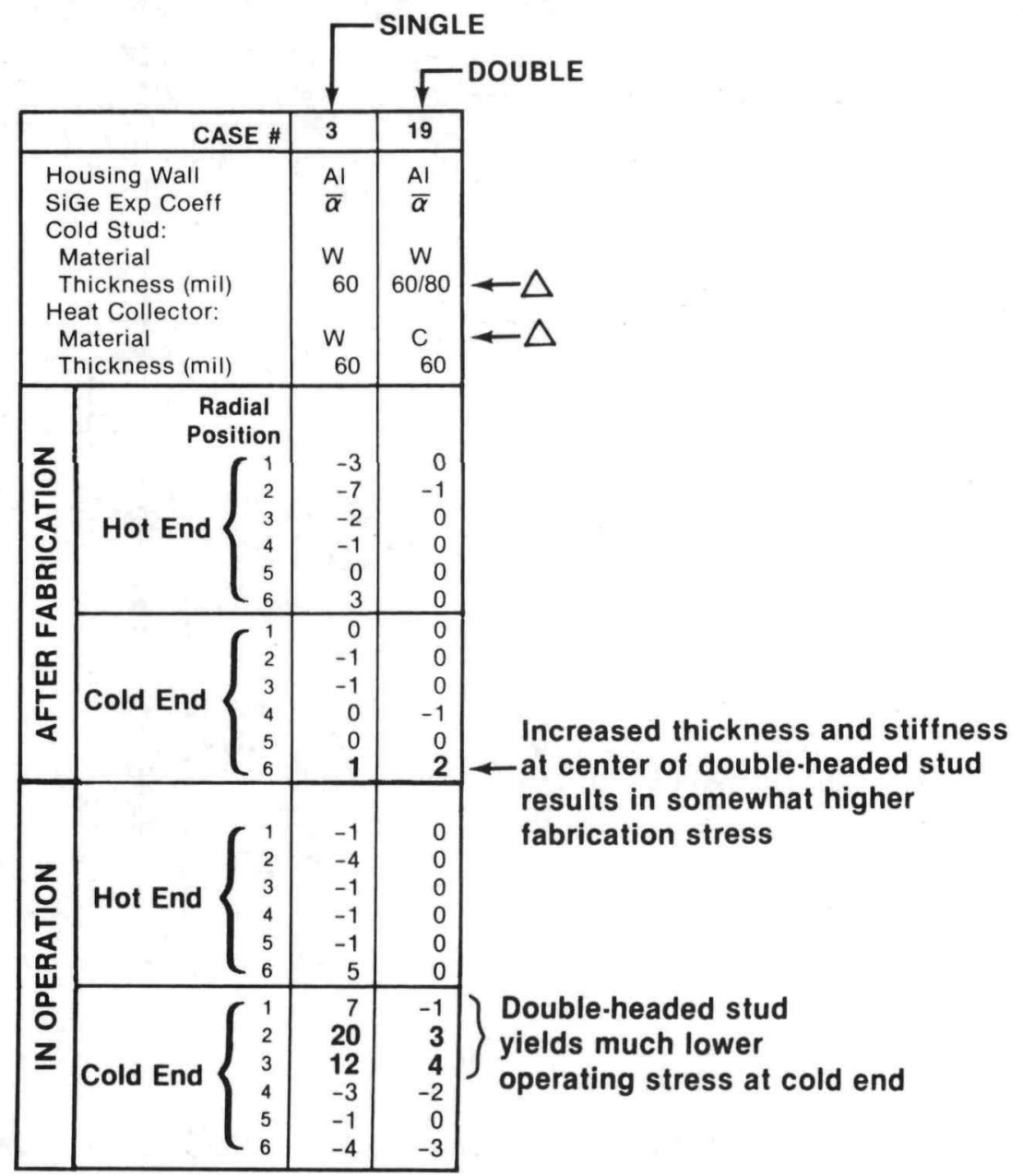

Thus, we see that the double-headed stud is much more effective than the graphite cushion in reducing the operating stresses at the center of the cold end. The effect of combining both of those design improvements is illustrated in Figure 13. 
Replacing 0.020" of the 0.060" upper studhead with graphite lowers the fabrication stress from $2 \mathrm{ksi}$ to $1 \mathrm{ksi}$; and, as shown in Figure 13, it reduces the peak cold-end stress from $4 \mathrm{ksi}$ to $2 \mathrm{ksi}$.

Figure 13. Double-Headed Tungsten Cold Stud with Graphite Cushion

OPERATING STRESSES (ksi) AT SiGe ENDS

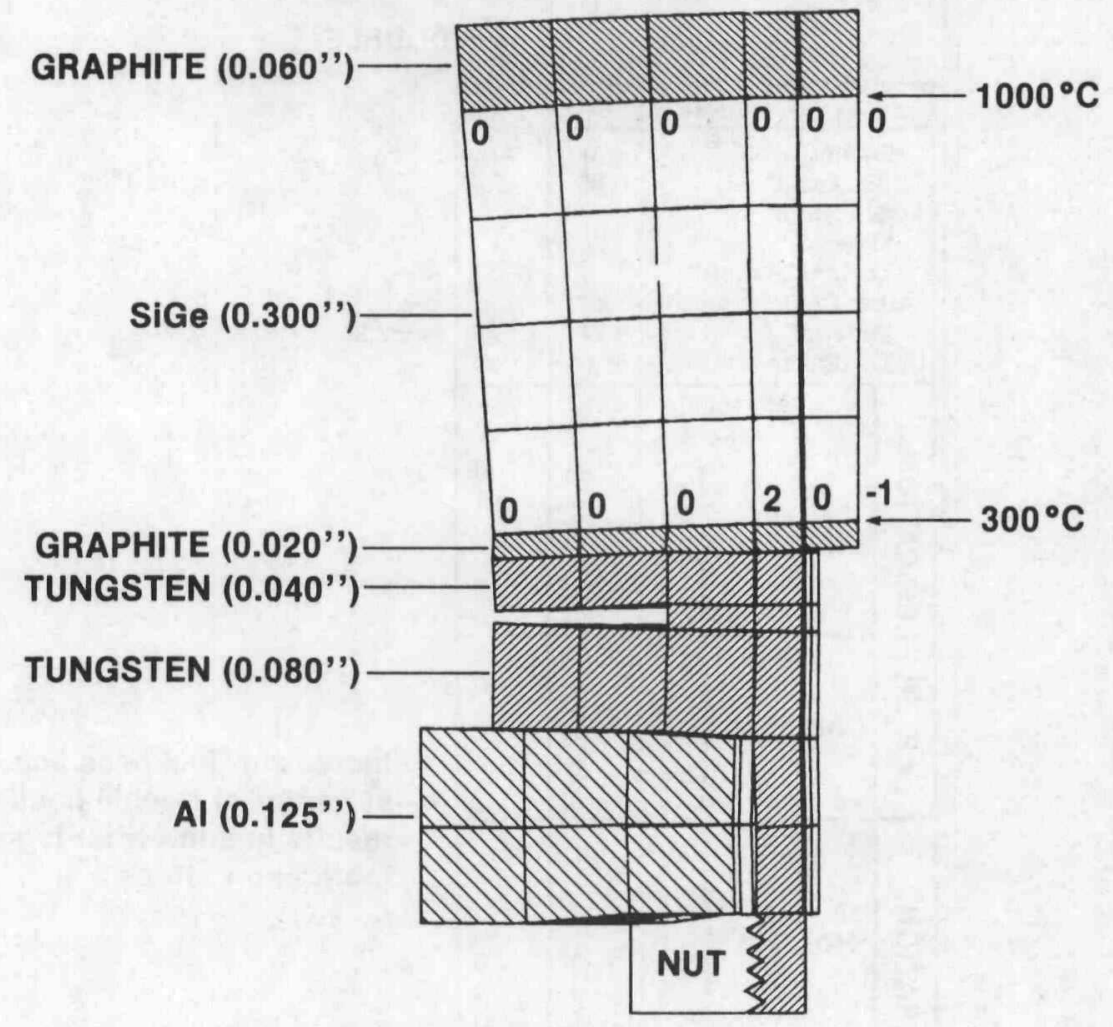

Additional analysis showed that even that value can be further cut in half by using $0.040^{\prime \prime}$ of graphite instead of $0.020^{\prime \prime}$. Thus, theory predicts that the combination of the double-headed stud with the compliant graphite cushion would virtually eliminate the cold-end stresses. 


\subsection{Graphite Heat Collector}

As suggested earlier, a heat collector maximum thickness of $0.060^{\prime \prime}$ is adequate for molybdenum but not for graphite. The thermal conductivity of the graphite used (Stackpole 2128) at $1000^{\circ} \mathrm{C}$ is $0.43 \mathrm{w} / \mathrm{cm}^{\circ} \mathrm{C}$, versus $1.15 \mathrm{w} / \mathrm{cm}^{\circ} \mathrm{C}$ for molybdenum. Thus, a graphite heat collector must be about 2.5 times as thick for the same thermal effectiveness. But since its density is only one sixth that of molybdenum, a graphite heat collector has less than half the weight of an equivalent molybdenum heat collector.

In addition to its lower weight and greater compliance, a graphite heat collector has three other advantages over molybdenum: It is easier to machine; it avoids the need for the troublesome W/Mo joint discussed in Section 4 of Reference [2]; and it avoids the need for a high-emissivity coating, since the emissivity of graphite is inherently high. Such a coating, which must last for at least seven years at operating temperature, would have been required in the case of a molybdenum heat collector, to avoid excessive heat source temperatures.

In fact, the heat source is constrained to a maximum surface temperature of $1100^{\circ} \mathrm{C}$, and this constraint dictates the required heat collector thickness profile. To determine the optimum thickness profile, Fairchild conducted an iterative series of three-dimensional thermal analyses. The minimum-weight solution for the prescribed heat flow and boundary temperatures is depicted in Figure 14. 
The optimum heat collector has the shape of " a very shallow pyramid or pagoda. The variation of its thickness $z$ as a function of its $x$ and $y$ dimension is indicated by the thermal-model plot in Figure 14. (Note the difference in scale.)

Figure 14. Isotherm Map of Graphite Heat Collector with Optimized Thickness Profile

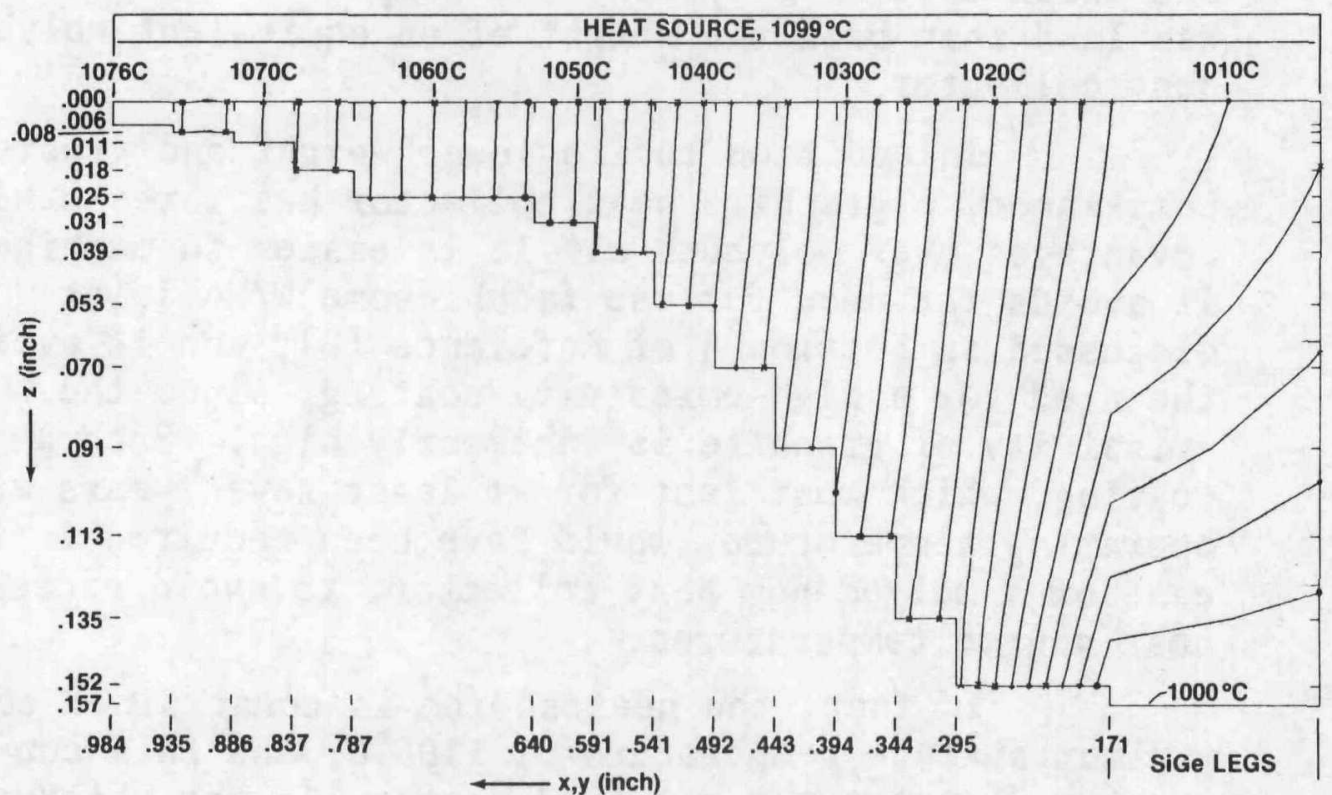

As shown, the resultant isotherms are fairly evenly spaced, which demonstrates that the shape is close to optimum. However, they are very widely spaced near the center, above the SiGe legs. This indicates that we have more graphite than needed in this region.

Figure 14 shows a maximum heat collector thickness of $0.157^{\prime \prime}$, compared to the previously used $0.060^{\prime \prime}$ value. To determine the effect of a thicker heat collector on the hot-end stresses, the case illustrated in Figure 13 was rerun with a $0.180^{\prime \prime}$ heat collector, with the results shown in Figure 15. 
Figure 15. Effect of 0.180" Graphite Heat Collector

OPERATING STRESSES (ksi) AT SiGe ENDS

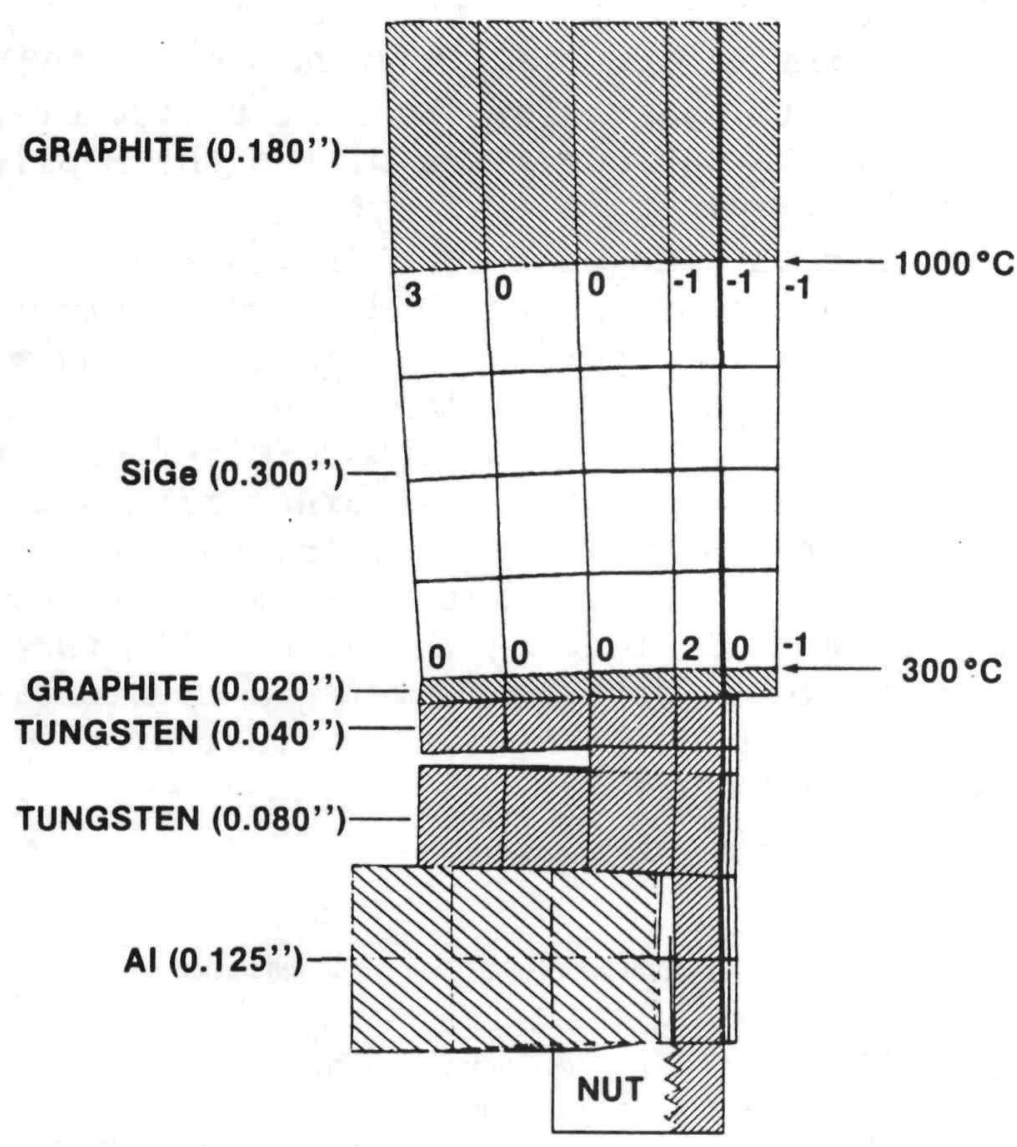

Comparison of Figures 13 and 15 shows that increasing the heat collector thickness from $0.060^{\prime \prime}$ to $0.180^{\prime \prime}$ has no effect on the cold-end stresses, but produces an operating tensile stress of $3 \mathrm{ksi}$ at the outer corner of the hot end. This is due to the increased stiffness of the thicker graphite plate. The stiffer heat collector resists conforming to the gradient-induced curvature of the thermopile shown in Figure 6. This results in compressive stresses near the center and tensile stresses at the outside of the interface. Thus, the replacement of W/Mo by an equivalent thickness of graphite has eliminated the fabrication stress at the hot end and has reduced the peak operating stress from $5 \mathrm{ksi}$ to $3 \mathrm{ksi}$, but has not eliminated it. 
While the remaining $3 \mathrm{ksi}$ stress may be tolerable, Fairchild suggested that this could be further reduced by cutting radial slots into the heater collector at $45^{\circ}$ intervals. This would have no effect thermally, but would make it easier for the heat collector to bend and to conform to the curvature of the thermopile caused by the temperature gradient.

To determine whether this would in fact reduce the tensile stress at the hot end's outer corner, the case illustrated in Figure 15 was modified analytically by assuming $45^{\circ}$ slots penetrating the full thickness of the heat collector. This was found to reduce the tensile stress at the hot end's corner from $3 \mathrm{ksi}$ to $1 \mathrm{ksi}$, but to raise the stress at the center from $-1 \mathrm{ksi}$ to $+2 \mathrm{ksi}$. To eliminate the latter stress Fairchild suggested slotting the heat collector to less than its full depth, and adding a countersink hole at its center. These changes are illustrated by the dashed lines in Figure 16.

Figure 16. Optimum Heat Collector Configuration

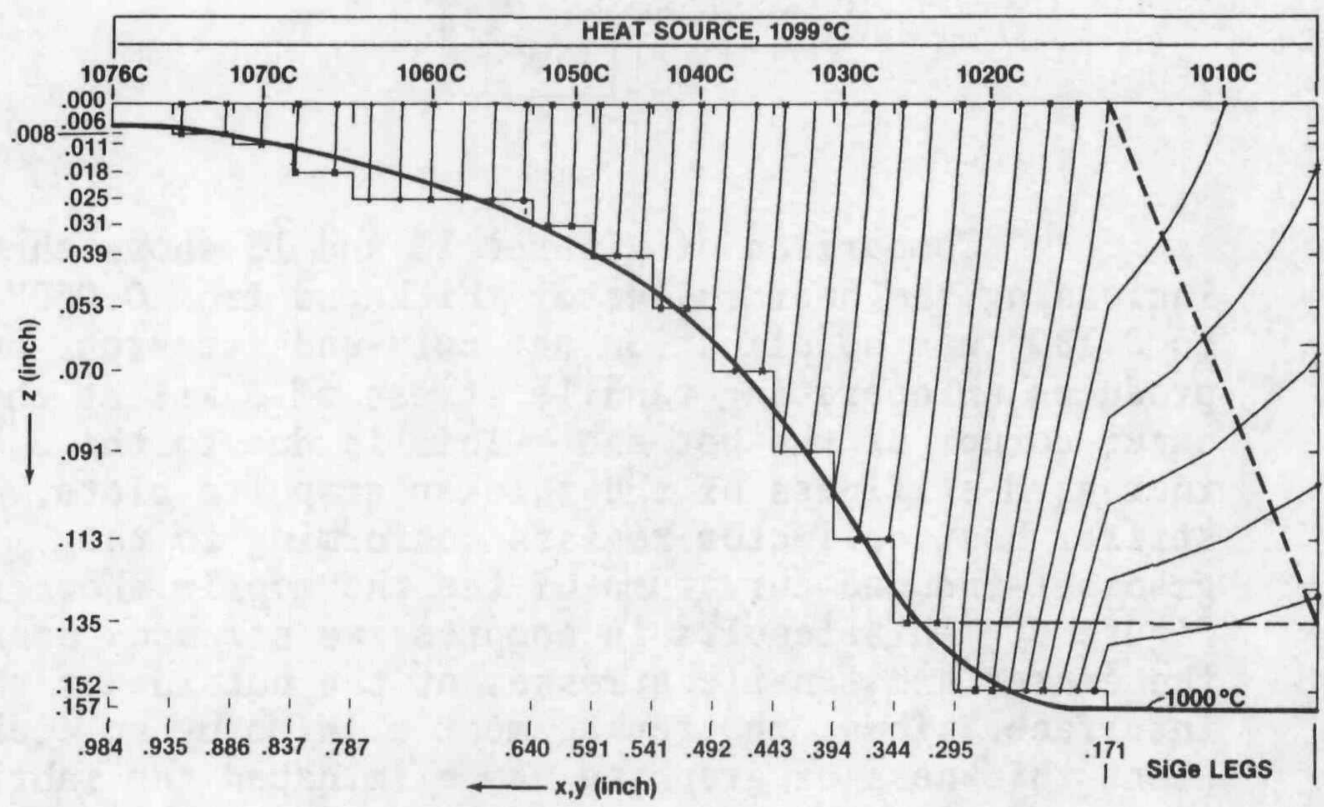


The reason for the countersink hole is that there is more graphite near the center of the heat collector than is needed. Removing this unneeded material by countersinking does not significantly affect the temperature distribution, but does reduce the heat collector's stiffness, which should further lower the hot-end stresses. To confirm this, a multicouple with the modified heat collector design depicted in Figure 16 was analyzed. This yielded the results shown in Table 9.

Table 9. Effect of Slotting and Countersinking the Graphite Heat Collector

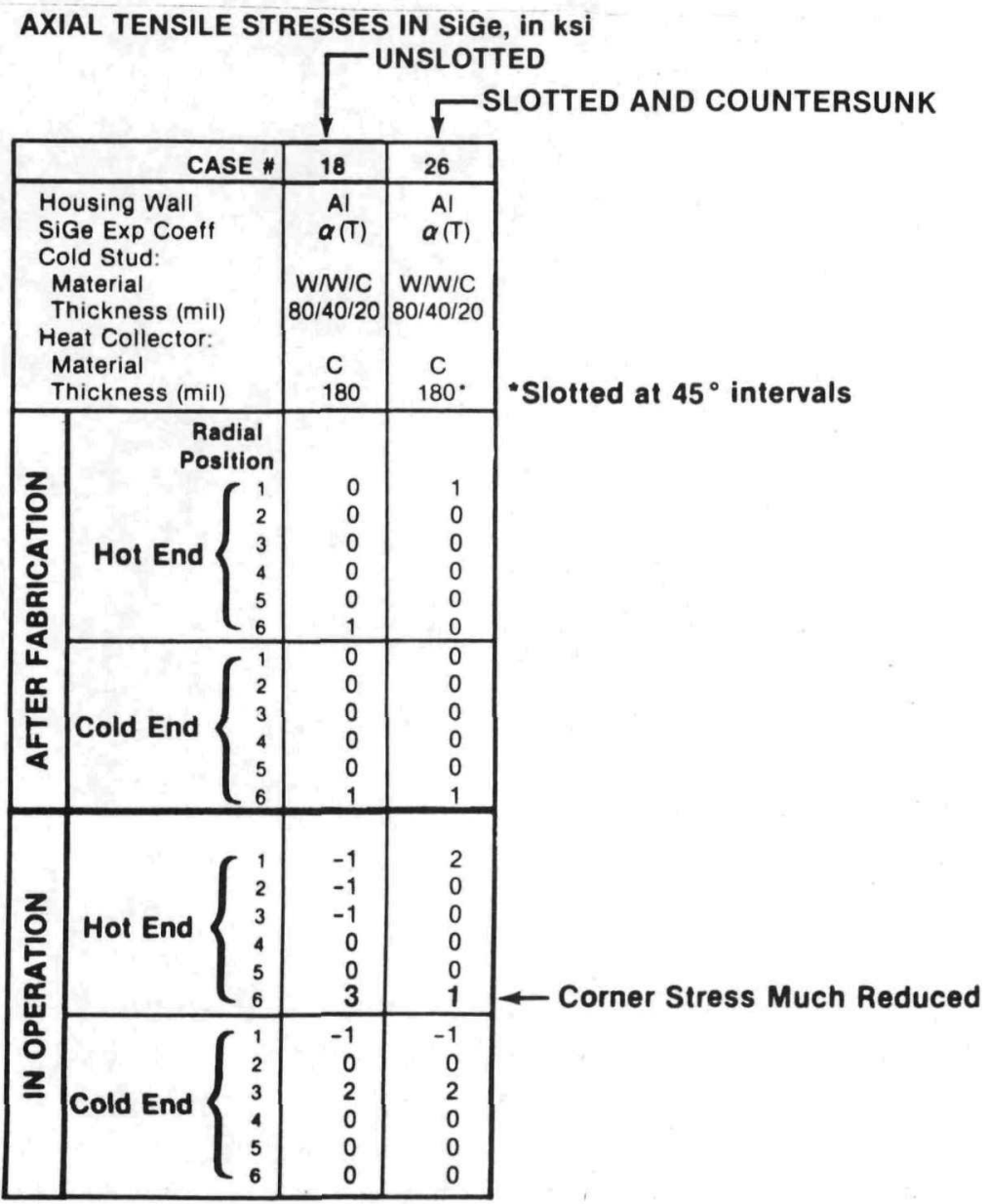


As can be seen, the modified heat collector design has eliminated the hot-end fabrication stresses, and has lowered the hot-corner operating stress from $3 \mathrm{ksi}$ to $1 \mathrm{ksi}$, without introducing new stresses elsetwhere. As depicted in Figure 17, a multicouple with a graphite heat collector incorporating the suggested slots and countersink has been built by Thermo Electron, and is undergoing evaluation tests.

Figure 17. Graphite Heat Collector

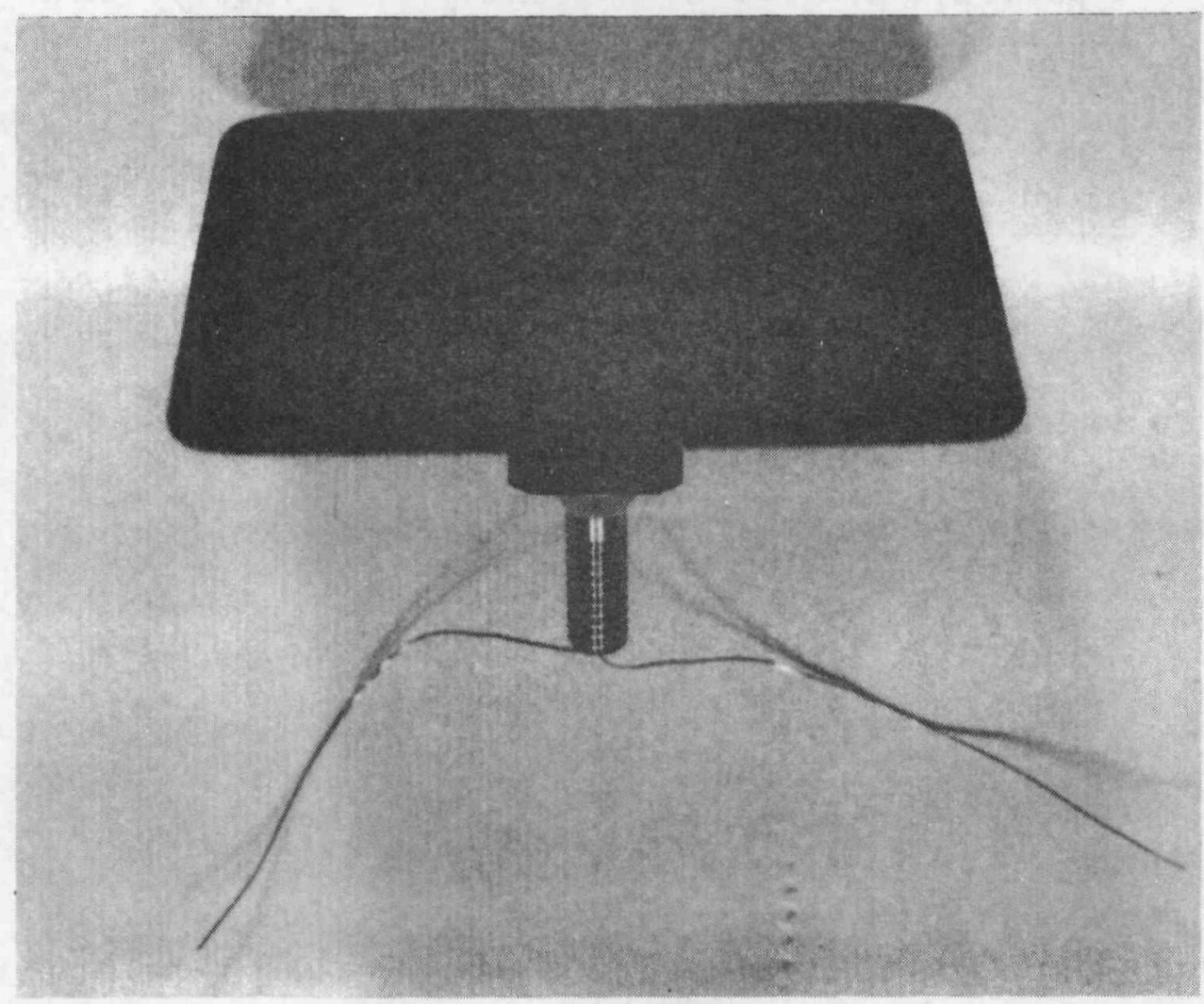

\subsection{Vibration Test}

The above analyses indicate that the use of a graphite cushion and graphite heat collector is beneficial in reducing thermal stresses in the SiGe thermopile. This makes it important to determine whether the graphite components can, in fact, be bonded to their neighbors; and whether these components and their neighbors will survive the predicted launch vibration loads. 
To help answer these questions, Thermo Electron fabricated a multicouple with a graphite cushion and heat collector, as illustrated in Figure 18. To bond the thermopile to the heat collector, its contact area was first coated with a $0.004 "$-thick layer of CVD tungsten. This was then bonded to the SiMo hot shoes by means of a high-temperature $\left(1100^{\circ} \mathrm{C}\right)$ glass layer, as in previous multicouples. In the case of the coldend graphite cushion, no tungsten coating was required, since the low-temperature glass $\left(680^{\circ} \mathrm{C}\right)$ was found to wet the graphite well. The lower face of the graphite cushion was bonded to the tungsten stud by means of a Nioro braze joint.

Figure 18. Multicouple with Graphite Components, for Performance and Vibration Tests

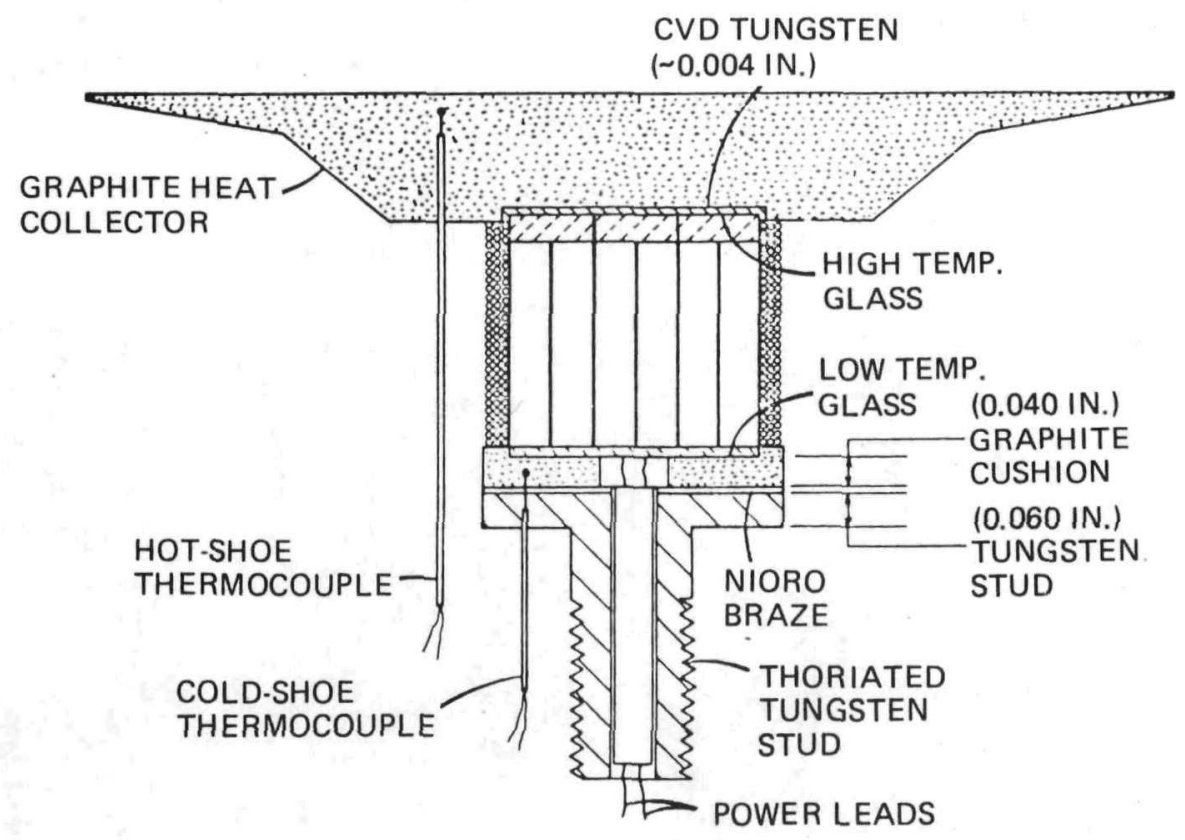

The above multicouple was first performancetested (at design temperature) at Thermo Electron, then vibration-tested at Fairchild, and then performancetested again at Thermo Electron. As can be seen, the multicouple employed a single-headed cold stud. To avoid the previously observed thermal stresses due to the aluminum wall expansion, the multicouple was tested without any wall. Instead, it was cooled by clamping a chill block directly to the stud pin. Its performance was found to be good, both before and after the vibration test, with no significant degradation. 
In the flight generator, the launch loads on the multicouple are not only those due to its own weight, but also those due to the weight of the multifoil insulation package, which is substantial. The multifoil insulation is supported only by the multicouples in the flight generator. During launch, this can result in significant additional shear loads and tensile loads on the SiGe thermopile and on the various multicouple bonds.

To simulate the effect of the multifoil loads, the vibration test designed by Fairchild employs a $2 \times 3$ multicouple array, supporting a corresponding multifoil package, as shown in Figure 19. Five of those multicouples are dummies, to simulate the foil support provided by neighboring elements. Only one (of the two middle ones) is a real multicouple.

Figure 19. Multicouple Vibration Test Fixture

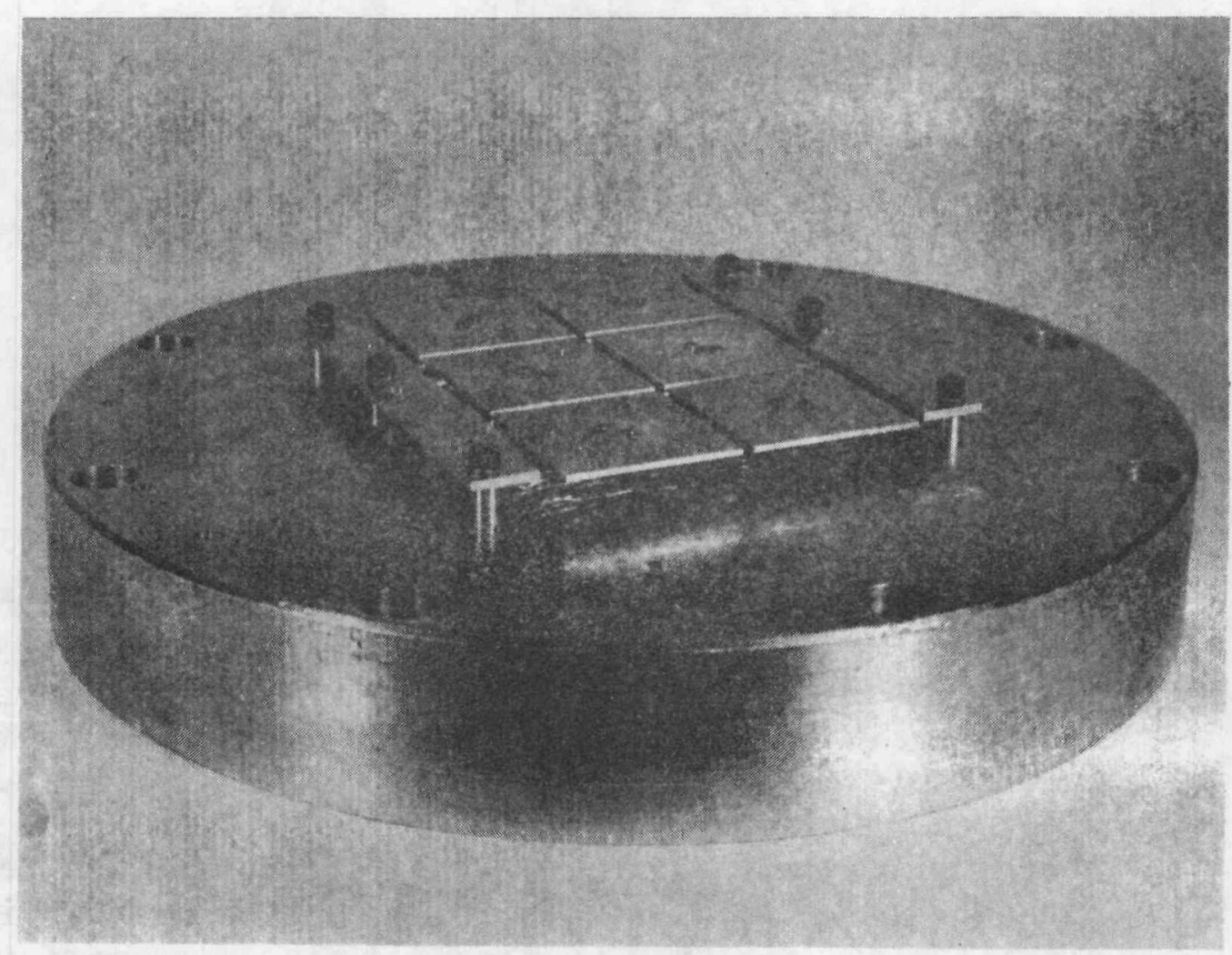


The multicouple depicted in Figure 18 was subjected to 2-minute random-vibration tests on three axes, based on currently predicted levels for the shuttle-launched Galileo and ISPM missions. Each of the three directions was subjected to an $8.11 \mathrm{grms}$ acceptance test and a 14.2 grms qualification test. In addition, the $z$-direction (parallel to the multicouple axis) was subjected to a 22.6 grms overtest. The power spectrum for that test is depicted by the jagged curve in Figure 20.

Figure 20. Multicouple Vibration Spectrum in 22.6 Grms Test

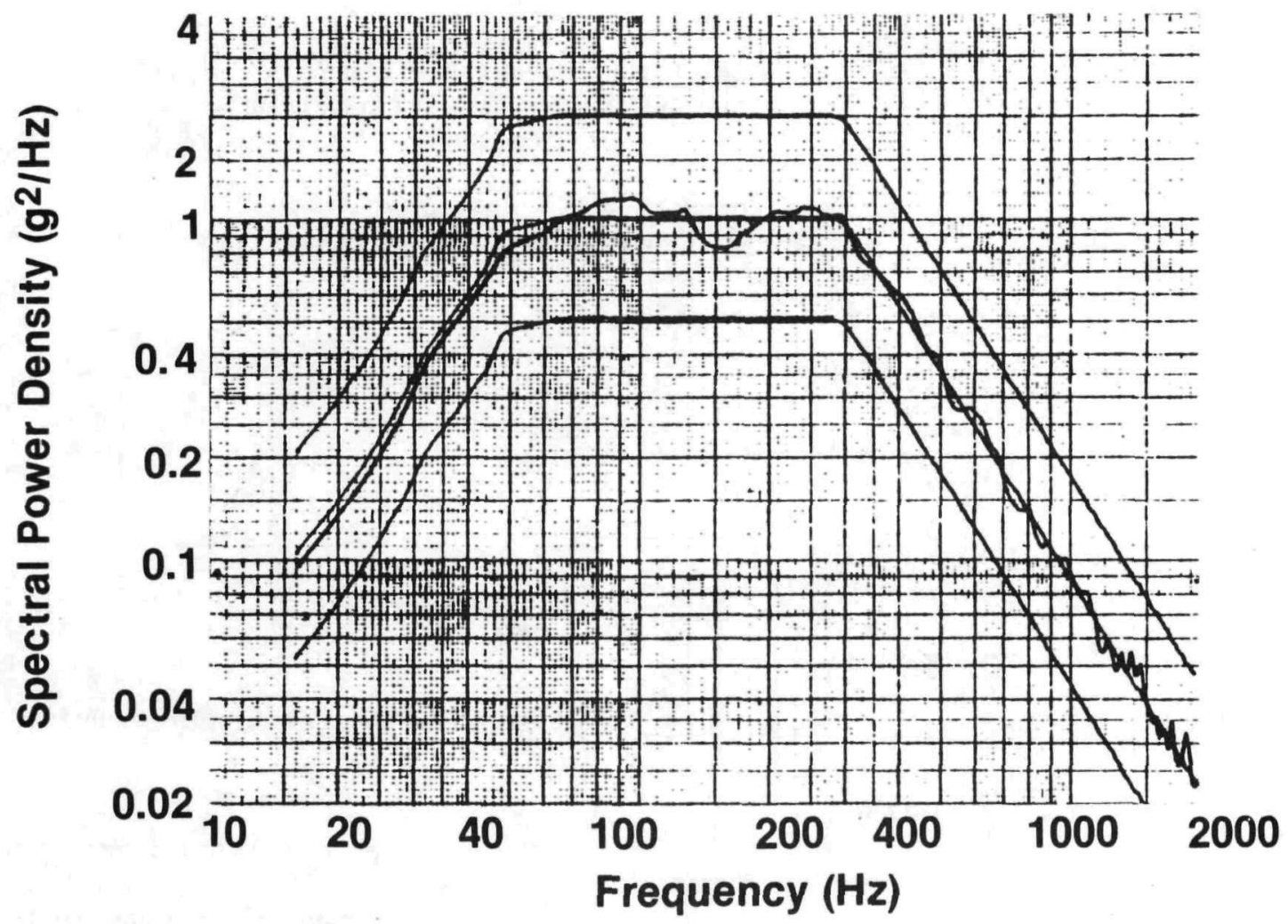

No damage was observed in the above tests, except for some minor fracture at the thin corner of the graphite heat collector. This can be avoided by truncating the corners in future heat collectors, without significant effect on multicouple performance. The in-gradient electrical performance of the tested multicouple was unchanged by the vibration test. 


\section{Summary}

The analysis described in this paper has identified the causes of the thermal-stress problems revealed by the preceding tests. The principal problem was shown to be the result of the high expansion coefficient of the aluminum housing wall. To alleviate these problems, the paper described a number of design changes, including the use of a double-headed stud with a $0.040^{\prime \prime}$ graphite cushion at the multicouple's cold end, and the use of a graphite heat collector with stress-relief slots at its hot end. The combined effect of these changes is summarized in Table 10.

Table 10. Total Improvement Over Base Case Axial Tensile Stresses in SiGe, in ksi

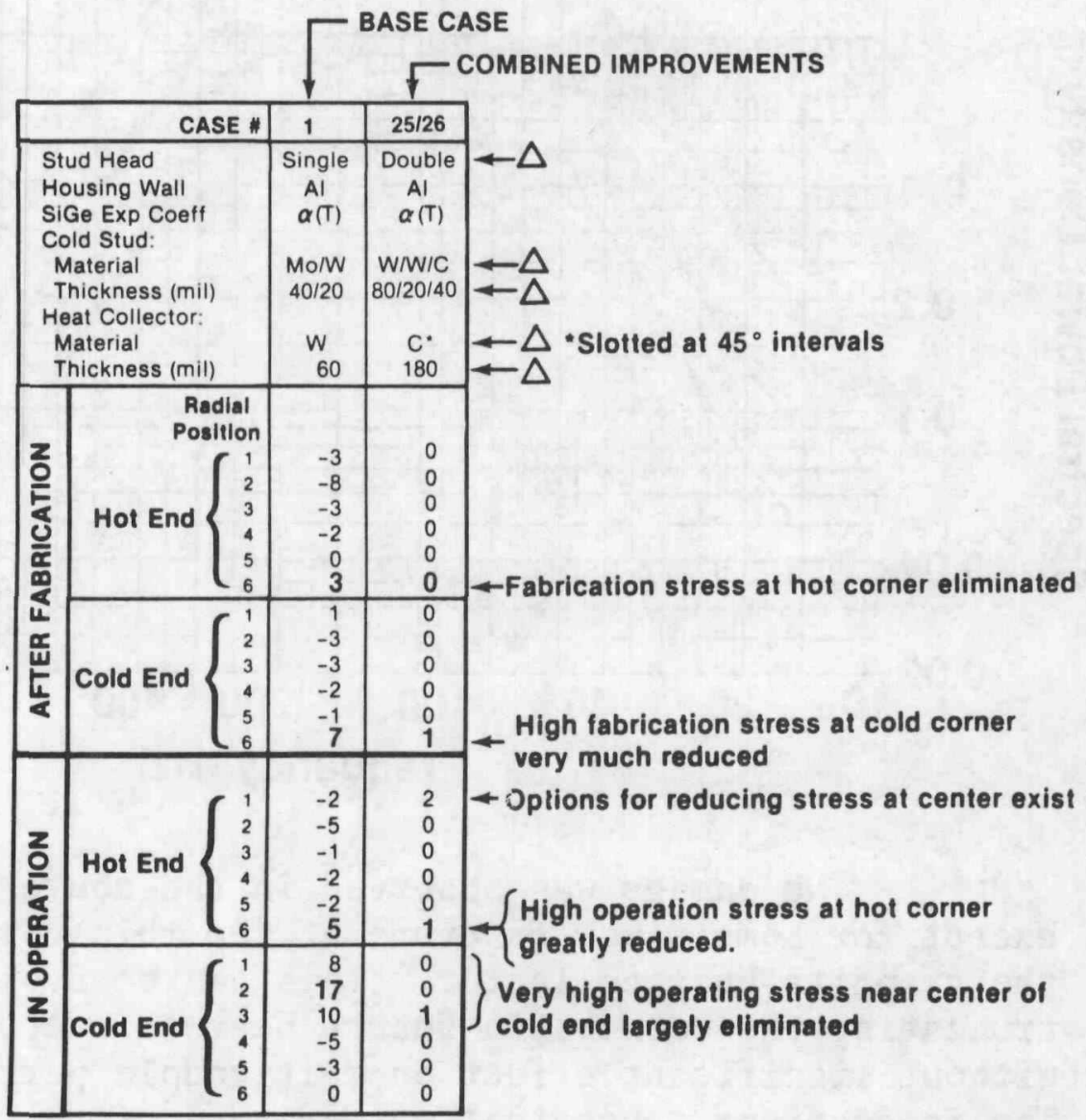


As can be seen, these design changes have virtually eliminated the previous fabrication stresses at the hot and cold corners, the previous operating stress at the hot corner, and the extremely high operating stress at the center of the cold end. Additional design changes for relieving these and some other problems are described in the next paper [4].

\section{Acknowledgment}

The author takes pleasure in acknowledging that the work described here reflects the contributions of many individuals, including (alphabetically): M. Eck, T. Kline, H. Kling, M. Mukunda, and J. Newell of Fairchild; and K. Hagen, F. Huffman, and V. Raag of Thermo Electron.

\section{References: (Part D):}

\section{Part:}

A. [1] A. Schock, "Modular Isotopic Thermoelectric Generator", Proceedings of the 1981 IECEC, Volume $1, \mathrm{Pg} .327$.

B. [2] A. Schock, "MITG Test Assembly Design and Fabrication", Proceedings of the 1983 IECEC, previous paper.

C.- [3] M. Eck, M. Mukunda, "MITG Test Procedure and Results", Proceedings of the 1983 IECEC, preceding paper.

E. [4] A. Schock, "Revised MITG Design, Fabrication Procedure, and Performance Predictions", Proceedings of the 1983 IECEC, next paper.

[5] J. Newel1, "Structural Analysis of a Thermoelectric Generator Element", MSC/NASTRAN User's Conference, Pasadena, Cal i fornia, March 1983. 
I

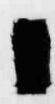

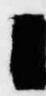

1

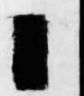

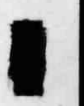

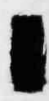

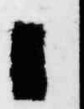

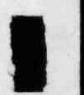

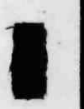

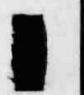

1

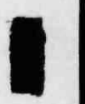

1

D42

1 


\title{
Part E:
}

\section{Revised MITG Design, Fabrication Procedure, and Performance Predictions*}

\author{
A. Schock \\ Fairchild Space Company \\ Germantown, Maryland 20874
}

(Presented at the 1983 Intersociety Energy Conversion Engineering Conference) 


\section{Abstract}

The design, analysis, and key features of the Modular Isotopic Thermoelectric Generator (MITG) were described in a 1981 IECEC paper; and the design, fabrication, testing, and post-test analysis of test assemblies simulating prototypical MITG modules were described in preceding papers in these proceedings. These analyses succeeded in identifying and explaining the principal causes of thermal-stress problems encountered in the tests, and in confirming the effectiveness of design changes for alleviating them.

The present paper presents additional design improvements for solving these and other problems, and describes new thermoelectric material properties generated by independent laboratories over the past two years. Based on these changes and on a revised fabrication procedure, it presents a reoptimization of the MITG design and computes the power-to-weight ratio for the revised design. That ratio is about $10 \%$ lower than the 1981-prediction, primarily because of changes in material properties and miscellaneous losses; but it is about 78 higher than the specific power of current-generation RTGs.

* The work reported in this paper was performed with the support of the Department of Energy's Office of Special Nuclear Projects, under the guidance of J. Lombardo, Director of Nuclear Systems Development. While the paper is the sole responsibility of its author, the work described represents the efforts of many contributors, from two companies, as indicated in the Acknowledgment Section. 


\section{Introduction}

The design of the Modular Isotopic Thermoelectric Generator (MITG), consisting of identical modular generator slices, was described [1] by the author at the 1981 Intersociety Energy Conversion Engineering Conference (IECEC). Since it offered a number of desirable features and a much higher power-to-weight ratio than current RTGs, DOE initiated a program to build and test such prototypical generator slices, primarily to measure their. power output and efficiency, and to determine their performance reproducibility and long-term stability.

The test design, results, and post-test analysis of the initial MITG tests are described in the preceding three papers in these proceedings. The first paper [2] describes the design and fabrication of the initial test assemblies. The second paper [3] describes the test plan, methodology, instrumentation, and the computer control and data acquisition system. And the third paper [4] describes detailed analyses to identify the causes of the observed thermal-stress problems, and to demonstrate that these can be eliminated by suitable design improvements.

The present paper describes and analyzes additional design improvement options, to solve the observed thermal-stress problems and to improve the thermal conductance and hermetic seal between the generator housing and the multicouple cold ends. It then describes a revised MITG design and fabrication procedure, analyzes its performance using updated thermoelectric material properties, and compares the results with those predicted in 1981 . 


\section{Multicouple Redesign}

\subsection{Double-Headed Cold Stud}

As discussed in Reference [4], the initial MITG tests revealed serious thermal-stress problems in the multicouples. Specifically, there was evidence of cracks at the center of the thermopiles' cold ends, and - to a lesser extent - at the corners of their hot and cold ends.

A detailed thermal-stress analysis of the original design yielded excellent agreement with the observed problems, and identified their causes. The principal problem was shown to be the high expansion coefficient of the aluminum housing wall.

Further analyses demonstrated that the observed fabrication and operating stresses could be virtually eliminated by using a multicouple with a graphitecushioned double-headed cold stud and a slotted graphite heat collector, as illustrated in Figure 1.

Additional design options for eliminating the thermal stresses and providing other improvements are described in the next two sections. 
Figure 1. Modified Multicouple with Double-Headed Cold Stud and Graphite Heat Collector

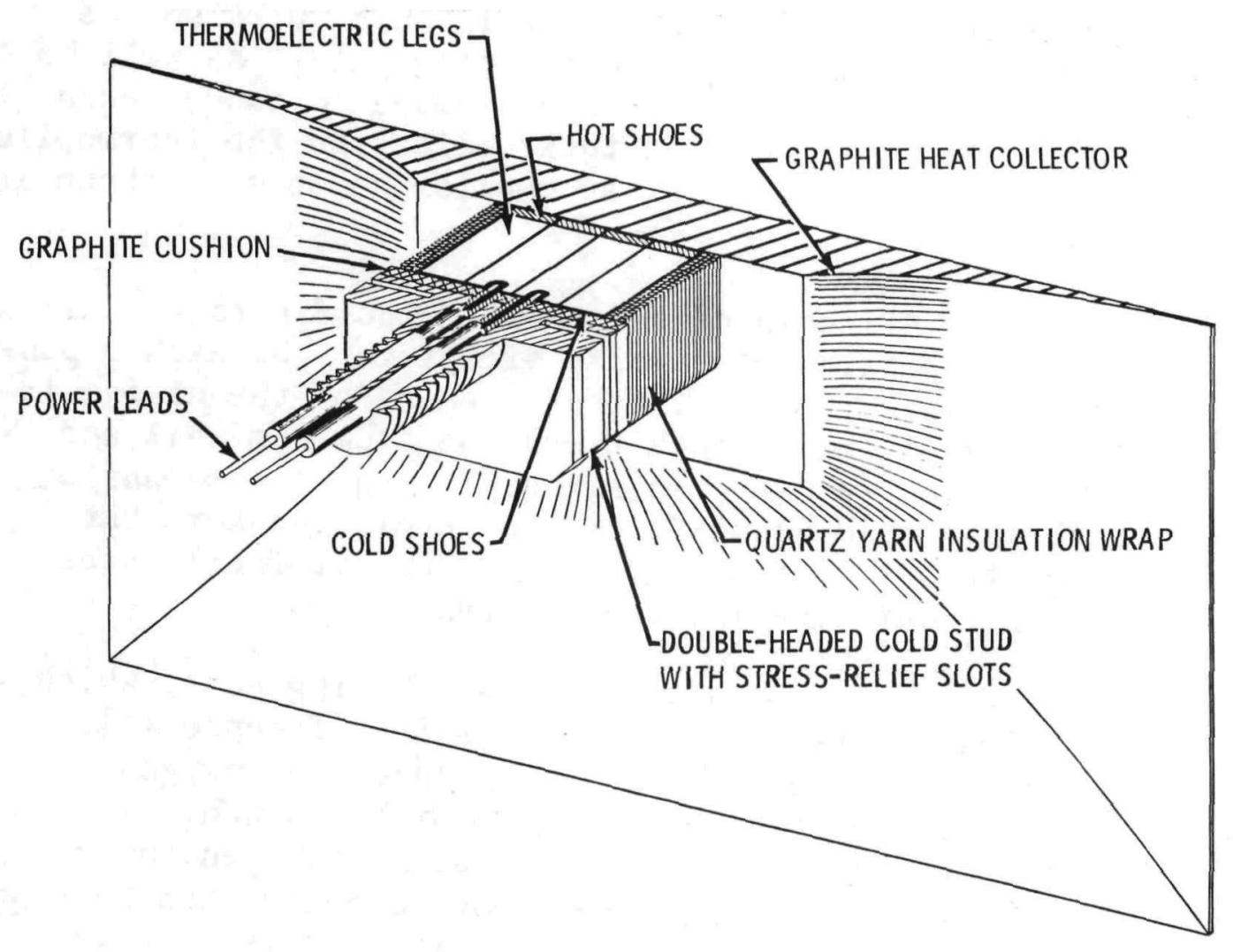




\subsection{Beveled Washer at Cold Stud}

Subsequent to the analysis of the doubleheaded cold-stud design described in Reference [4], the author thought of an alternative concept for avoiding the cold-end stresses produced by the thermal expansion of the aluminum wall. That concept is based on the fact that the expansion of the aluminum increases not only the wall thickness but also the radius of the multicouple mounting hole. As will be shown, these two effects can be made to cancel each other, which eliminates their effect on the thermopile's cold end. A design for accomplishing this is shown in Figure 2.

Al1 components shown in the figure are made of low-expansion materials, except for the high-expansion aluminum wall. The key element of the design is the conical interface between the aluminum wall and the beveled washer. In principle, any low-expansion material could be used for the beveled washer, but graphite has been called out because it facilitates sliding motion during thermal cycling.

Figure 2 also depicts a C-ring seal, which had been omitted in most figures in Reference [4]. A seal is needed to prevent the influx of oxygen during atmospheric operation prior to launch. In Reference [1], an axial C-ring seal had been shown on the inside of the housing, between the stud head and the wall. The C-ring shown in Figure 2 is a radial seal, between the stud pin and the housing hole. Its accessibility from outside the housing makes it possible to replace any defective seal without opening the converter and removing the affected multicouple. 
Figure 2. Use of Beveled Washer with Single-Headed Tungsten Stud

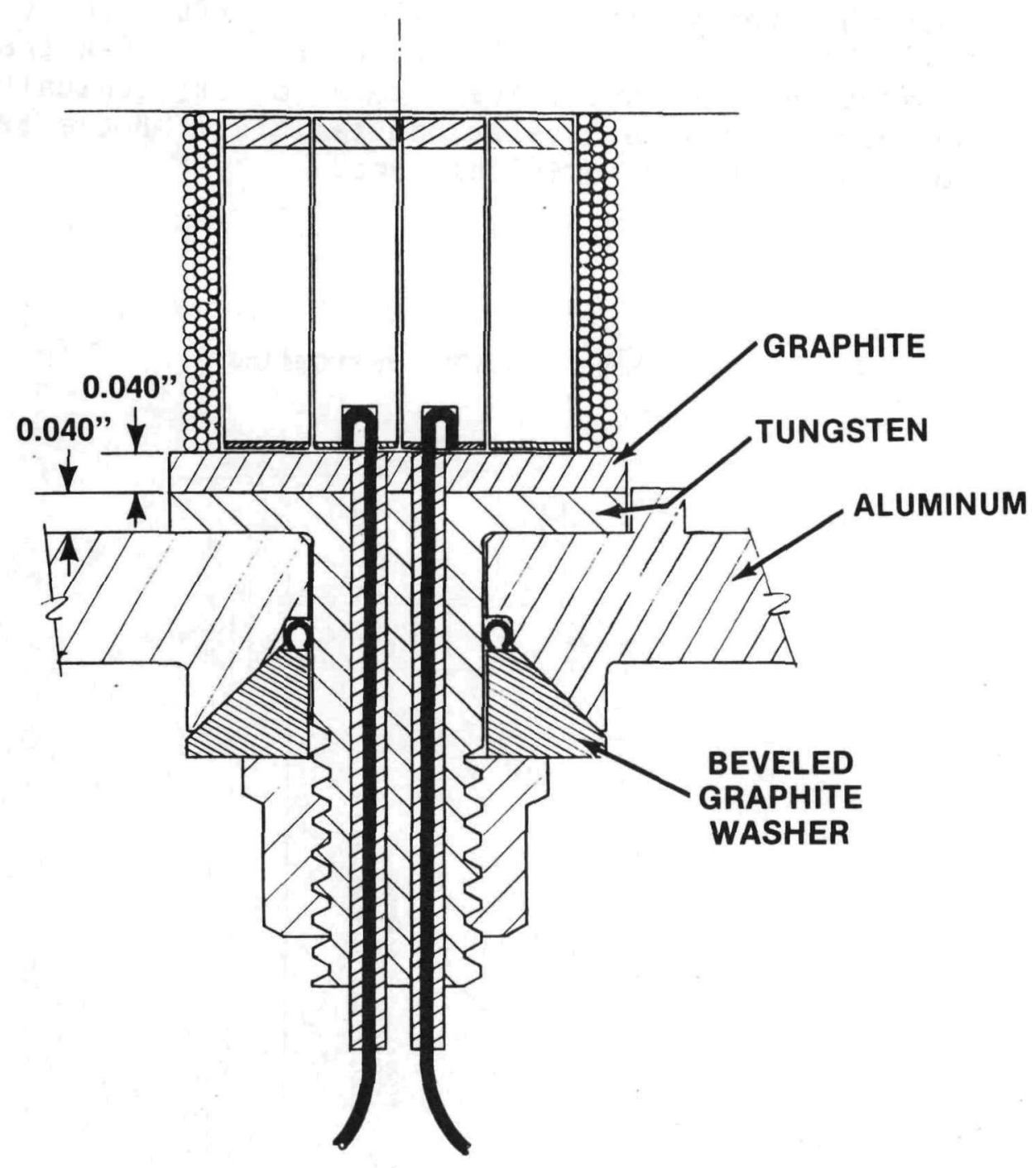


The key feature of the beveled-washer design is that both load-bearing surfaces of the wall have a common reference point, as illustrated in Figure 3 . Note that the apex of the conical interface coincides with the center of the planar interface. When the aluminum wall expands, every point on its conical surface moves both axially and radially. But every point stays on the original cone.

Figure 3. Close-Up View of Cold End

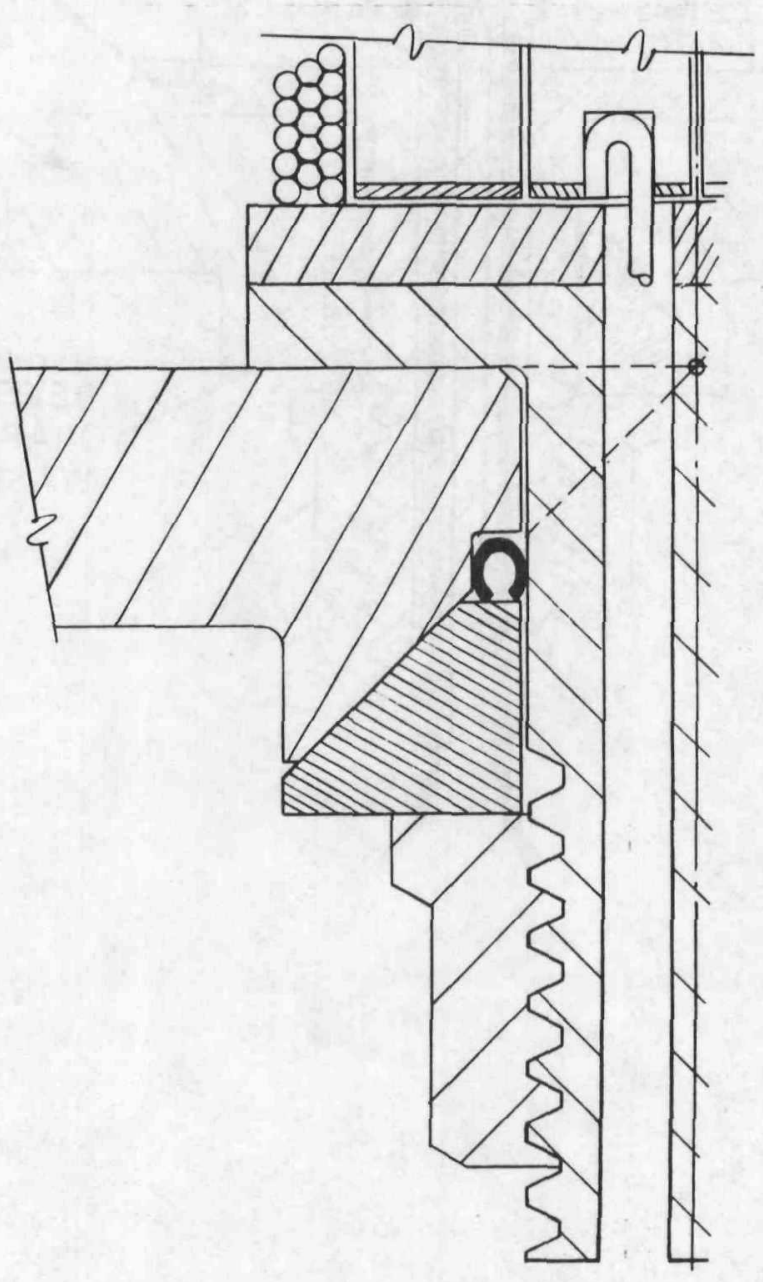


This is illustrated in Figure 4, where the dashed lines represent the expanded position of the wall. (The thermal expansion from room temperature to operating temperature has been magnified by a factor of ten, to improve visibility.) As can be seen, thermal growth of the aluminum wall increases its thickness, but does not affect the spacing between the stud head and the beveled washer. Therefore, it does not produce any stress in the SiGe thermopile. One advantage of the beveled-washer design is that it avoids the relatively high temperature drop of the double-headed stud design.

Figure 4. Effect of Aluminum Expansion

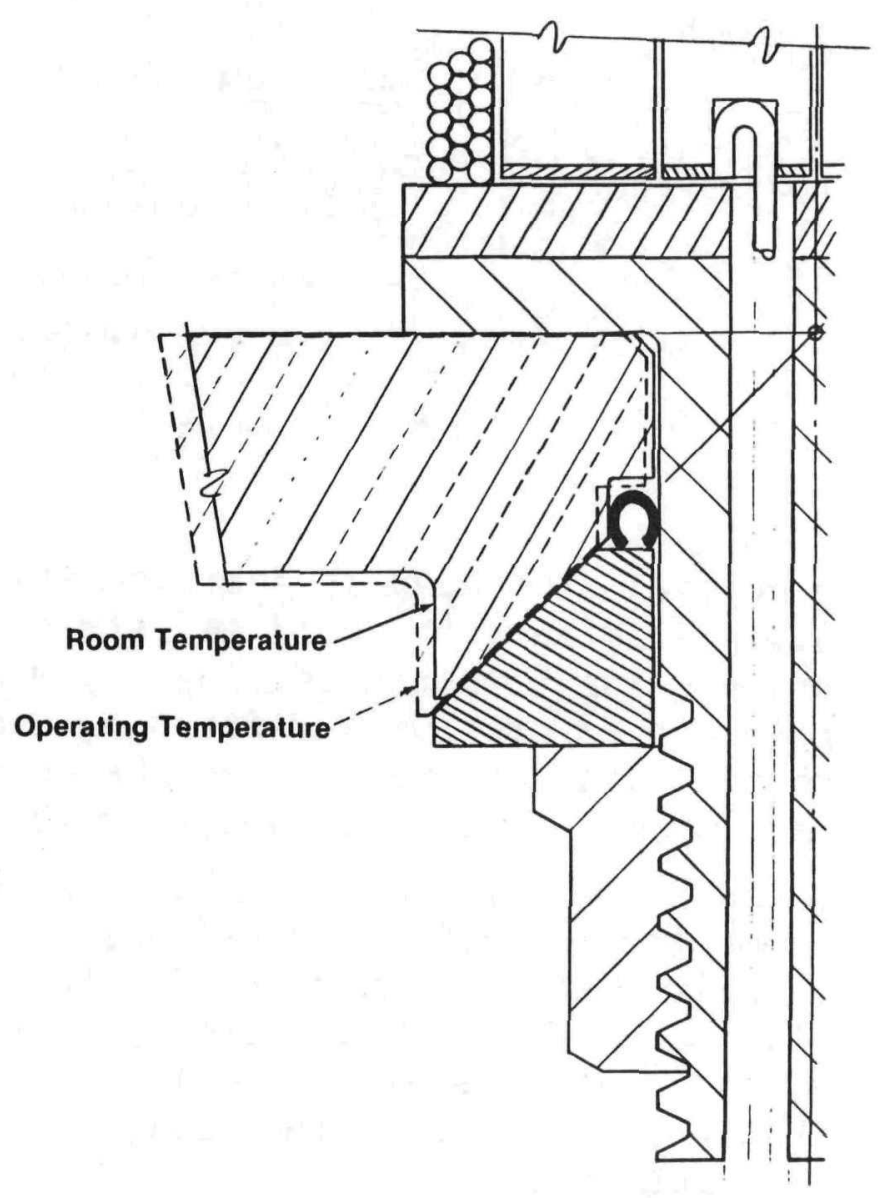




\subsection{Conical Stud Head}

Still another design option for eliminating the cold-end thermal stress was proposed by Thermo Electron. The key element of that design is the use of a conical cold-stud head, as illustrated in Figure 5 .

Figure 5. Multicouple With Conical Stud Head

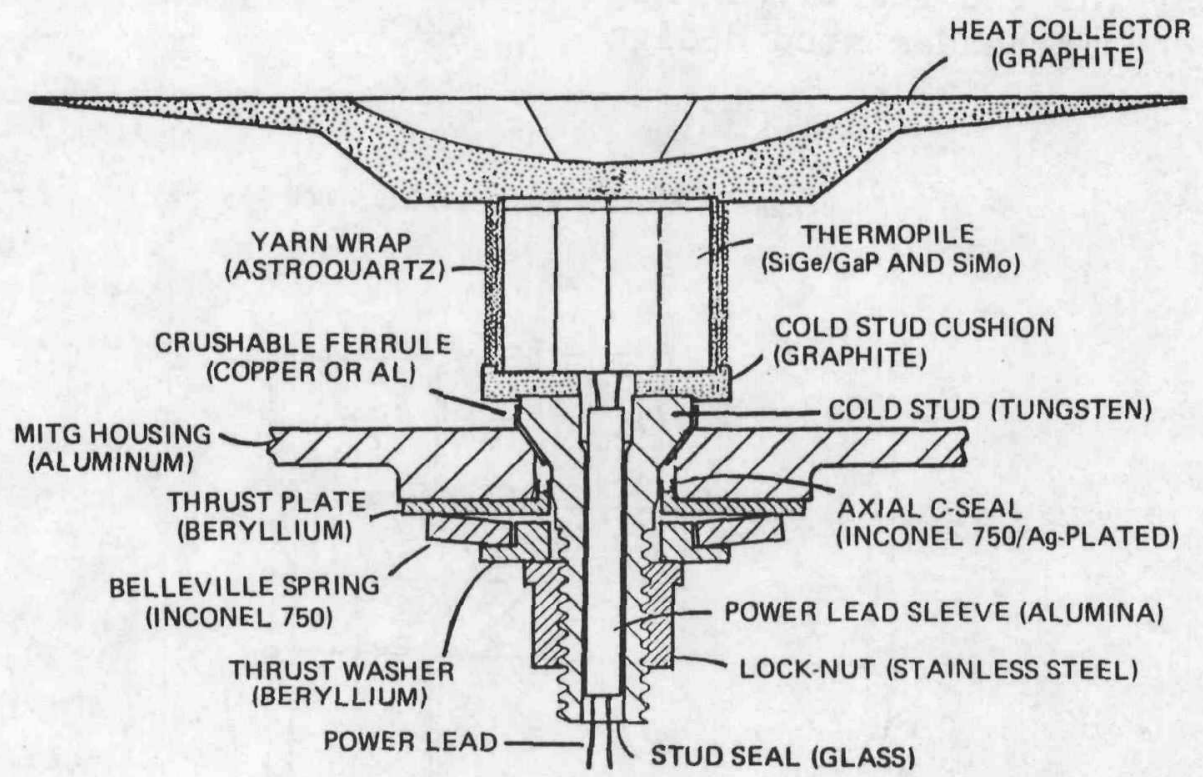

The conical stud head is designed to serve three functions: eliminate the cold-end stress due to wall expansion; provide hermetic sealing, by means of a soft crushable copper or aluminum ferrule; and improve the thermal contact conductance between the cold stud and the housing, by means of that same ferrule.

The crushable ferrule is conical, with a short cylindrical pilot for centering and alignment. It is annealed to make it dead-soft, and is identical to similar ferrules used to seal tube fittings. As shown in the figure, the conical seal can - if required be backed up by an additional C-ring seal, accessible from outside the housing. 
The operation of the seal depends on sufficient axial force being applied to the threaded stud to crush the conical ferrule. The resultant flow of metal compensates for surface roughness and asperities, producing a good seal and good thermal contact.

As shown in Figure 5, the seal is loaded by means of an Inconel-750 Belleville spring. The spring compensates for the thermal expansion of the aluminum housing wall. Since one of the load-bearing surfaces is conical, the thermal expansion effect would in any case be very small, because the conical interface - in effect - serves the same function as the beveled washer discussed in Section 2.2.

An incidental benefit of the Belleville spring is that its compression during installation of the multicouple in the housing serves as a measure of the applied preload. This avoids the uncertainties of torque measurements, which are subject to friction effects at the threads.

Figure 5 shows the Belleville spring bearing against a beryllium thrust washer and thrust plate, to prevent galling of the aluminum wall. But, as pointed out by Thermo Electron, these parts could probably be eliminated by using a specially designed Belleville spring with chamfered or rounded load-bearing edges.

Figure 5 depicts the slotted and counter-sunk graphite heat collector discussed earlier in Section 6.3 of Reference [4]. It also shows a compliant graphite cushion between the cold stud and the SiGe assembly. As was explained, this serves to reduce fabrication and operating stresses at the thermopile's cold end. 
Operating stresses at the cold end should be minimal in this design, because the Belleville spring accommodates the thermal expansion of the aluminum wall. But significant fabrication and/or operating stresses may result from the unusual thickness and stiffness of the tungsten cold stud. To determine whether this is the case, the design depicted in Figure 5 was analyzed, using the analytical procedure discussed in References [4] and [5], and the structural model shown in Figure 6 .

Figure 6. Analytical Model of Conical Stud-Head

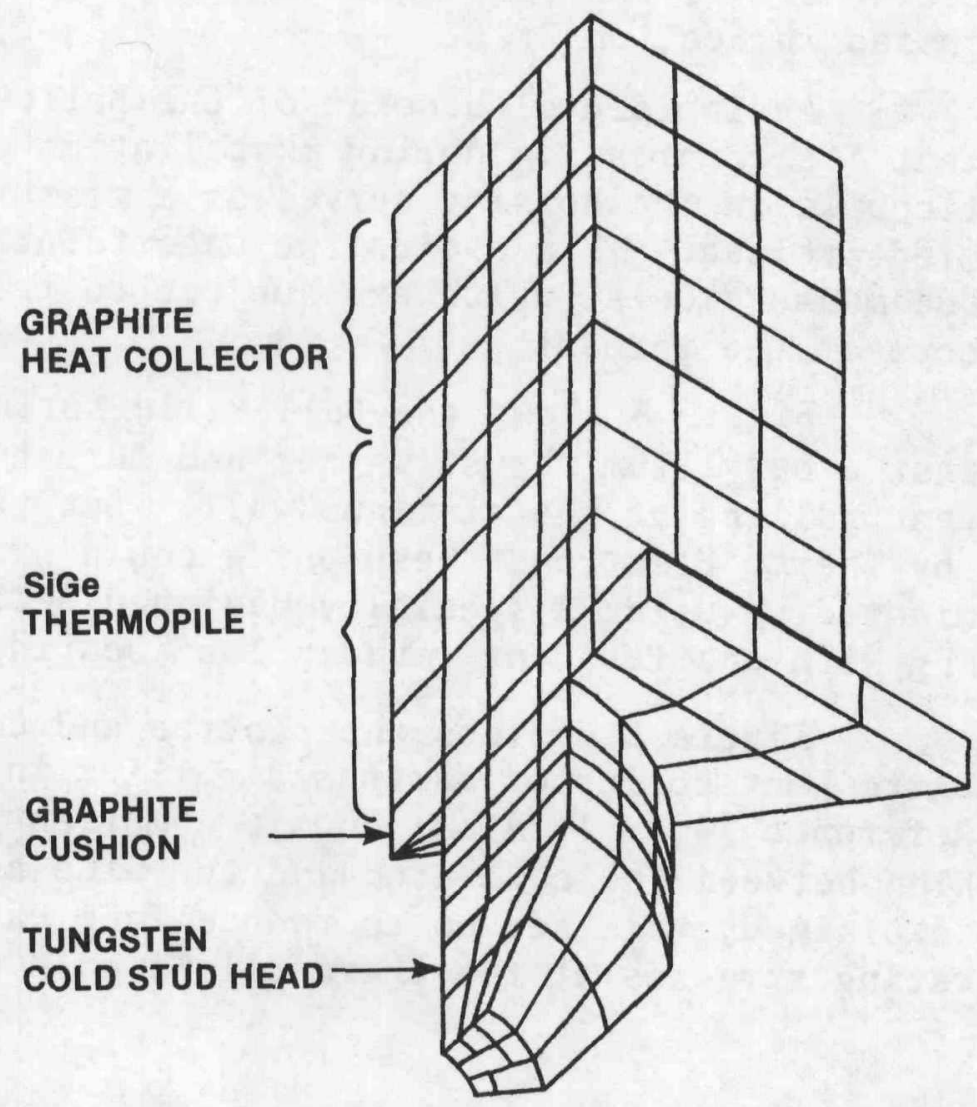


As can be seen, the model consists of a $45^{\circ}$ segment of the graphite heat collector, the $\mathrm{SiGe}$ thermopile, the graphite cushion, and the tungsten coldstud head. Its conical surface bears against a mating seat in the aluminum housing (not shown), and sliding was permitted at the conical interface. The Belleville spring force was applied as a uniform tensile load on the bottom face of the cold-stud pin.

The results of the analysis indicated very low fabrication and operating stresses, less than $1 \mathrm{ksi}$, at all points of the SiGe cold end.

In parallel with the above analysis, Thermo Electron is conducting a number of tests on this design. They have succeeded in machining the threaded cold stud out of arc-cast tungsten. This material has a very high tensile strength, much higher than the previously used molybdenum studs. The relative weakness of the previous stud pins had seriously limited the torque that could be applied to produce good thermal contact between the stud head and the wall.

Tensile tests of the machined tungsten stud indicate it has more than enough strength to crush the conical ferrule shown in Figure .5. This has been confirmed in sealing tests, both on copper ferrules and aluminum ferrules. Excellent hermetic seals were obtained, at spring loads far below the strength limit of the threaded stud, and well within the capability of the Belleville spring. 
Next, a cold-end thermal analysis of the conical-stud-head design was performed by Fairchild. As shown in Figure 7 , the 1334-node rz-thermal model extended from the SiGe cold end to the outer edge of the aluminum wall. It included the .002"-thick glass bond layer, the $0.020^{\prime \prime}$ graphite cushion (above the dashed line), the tungsten cold-stud head, and the aluminum wall section. The diagonal line of small rectangles was inserted to simulate the contact resistance at the conical interfaces on both sides of the ferrule.

Figure 7. Isotherm Map of Cold End with Conical Stud Head

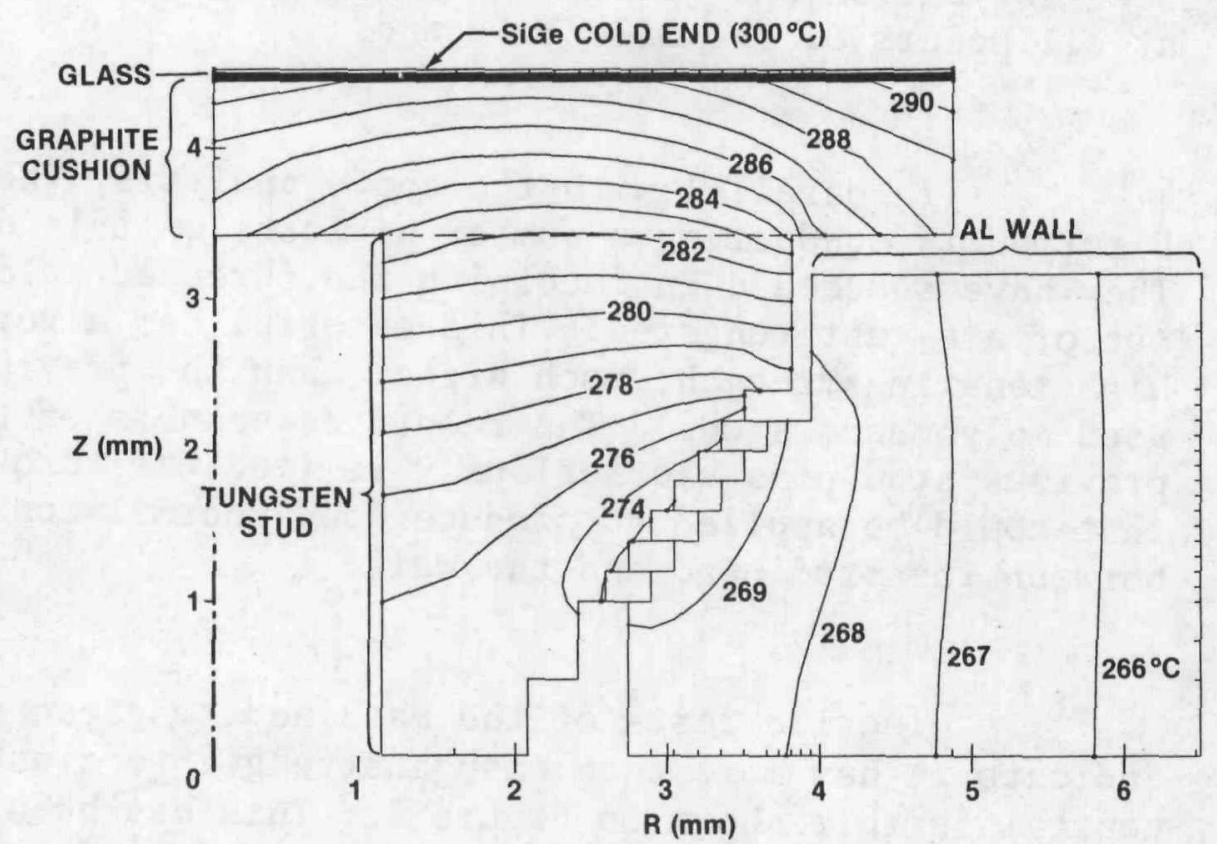

The results. shown are, of course, quite sensitive to the assumed contact resistance at the conical interfaces. Experiments at Thermo Electron to determine that resistance gave very encouraging results. They yielded a combined contact resistance of $0.035^{\circ} \mathrm{C}$ per $w / \mathrm{cm}^{2}$ for the two interfaces on both sides of the crushable ferrule. Use of that value in Fairchild's thermal analysis gave the results shown in Figure 7. The very low contact resistance measured is attributable to the metal flow of the soft aluminum ferrule under the applied spring load. 
As shown in Figure 7 , there is a temperature drop from $300^{\circ} \mathrm{C}$ at the SiGe cold junctions to $269^{\circ} \mathrm{C}$ at the mounting hole of the aluminum wall. The total coldend drop of $31^{\circ} \mathrm{C}$ is very close to the value of $30^{\circ} \mathrm{C}$ postulated in the author's 1981 paper [1]. Note that $10-12^{\circ} \mathrm{C}$ of the $31^{\circ} \mathrm{C}$ drop is due to the cold-end glass bond, and $5^{\circ} \mathrm{C}$ is due to the contact resistance.

Thermo Electron is currently fabricating complete multicouples of the design shown in Figure 5. One of these, including a graphite heat collector, graphite cushion, and tungsten cold stud, is depicted in Figure 8.

Figure 8. Multicouple with Conical Tungsten Stud and Graphite Heat Collector and Cushion

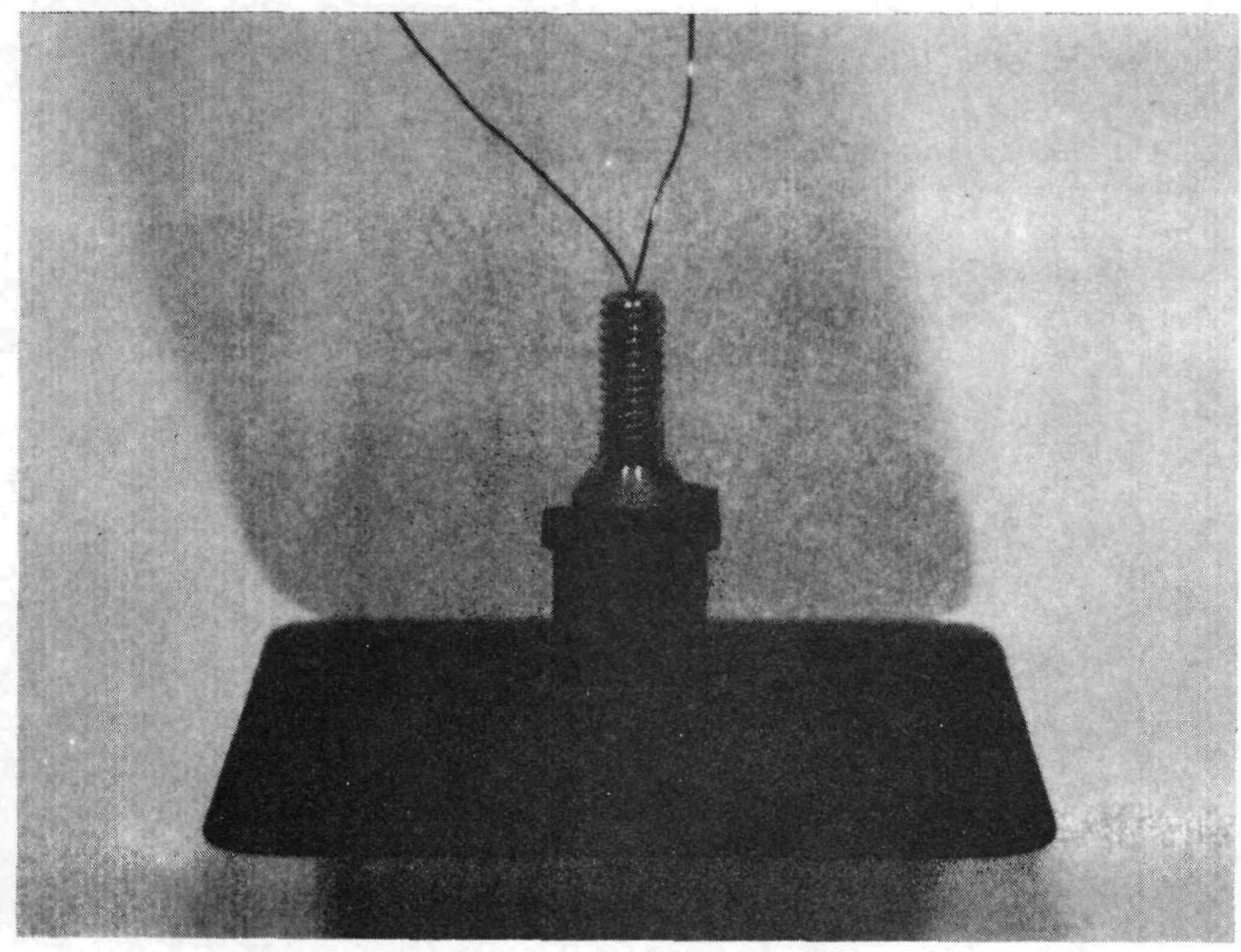

The above multicouples are undergoing performance tests at Thermo Electron, with very encouraging results. After these are confirmed, a full complement of eight will be fabricated by them and delivered to Fairchild for installation and testing in a complete test assembly simulating a prototypical slice of the MITG. 


\section{Updated MITG Design}

The author's 1981 paper [1] had presented detailed weight and performance estimates for a typical flight generator. Since then, there have been a number of design changes and analytical refinements, as described in the present paper and in Reference [4]. In addition, various laboratories (Purdue, Battelle, Ames) have, at DOE's behest, carried out independent measurements of the properties of the $\mathrm{SiGe/GaP}$ material developed by Syncal. The resultant set of agreed-upon properties differs appreciably from Syncal's original measurements.

Since the above changes could have a significant effect on the performance of the flight generator, Fairchild was asked to reoptimize the MITG design based on the latest design concepts and material properties, and to reevaluate its predicted power-to-weight ratio.

\subsection{Material Properties Changes}

The changes in properties of $\mathrm{SiGe/GaP}$ since the author's 1981 paper [1] are displayed in Figure 9, which shows the temperature dependence of the material's Seebeck coefficient, electrical resistivity, and thermal conductivity.

The figure compares Syncal's earlier measurements with the currently accepted measurements by Ames and Purdue (A/P). As can be seen, the Seebeck coefficient of the p-leg has dropped significantly, but this is partially offset by its lower electrical resistivity. In addition, Figure 9 shows that the thermal conductivity of $\mathrm{SiGe/GaP}$ has increased appreciably, but is still significantly lower than that of SiGe. 
Figure 9. Thermoelectric Property Changes
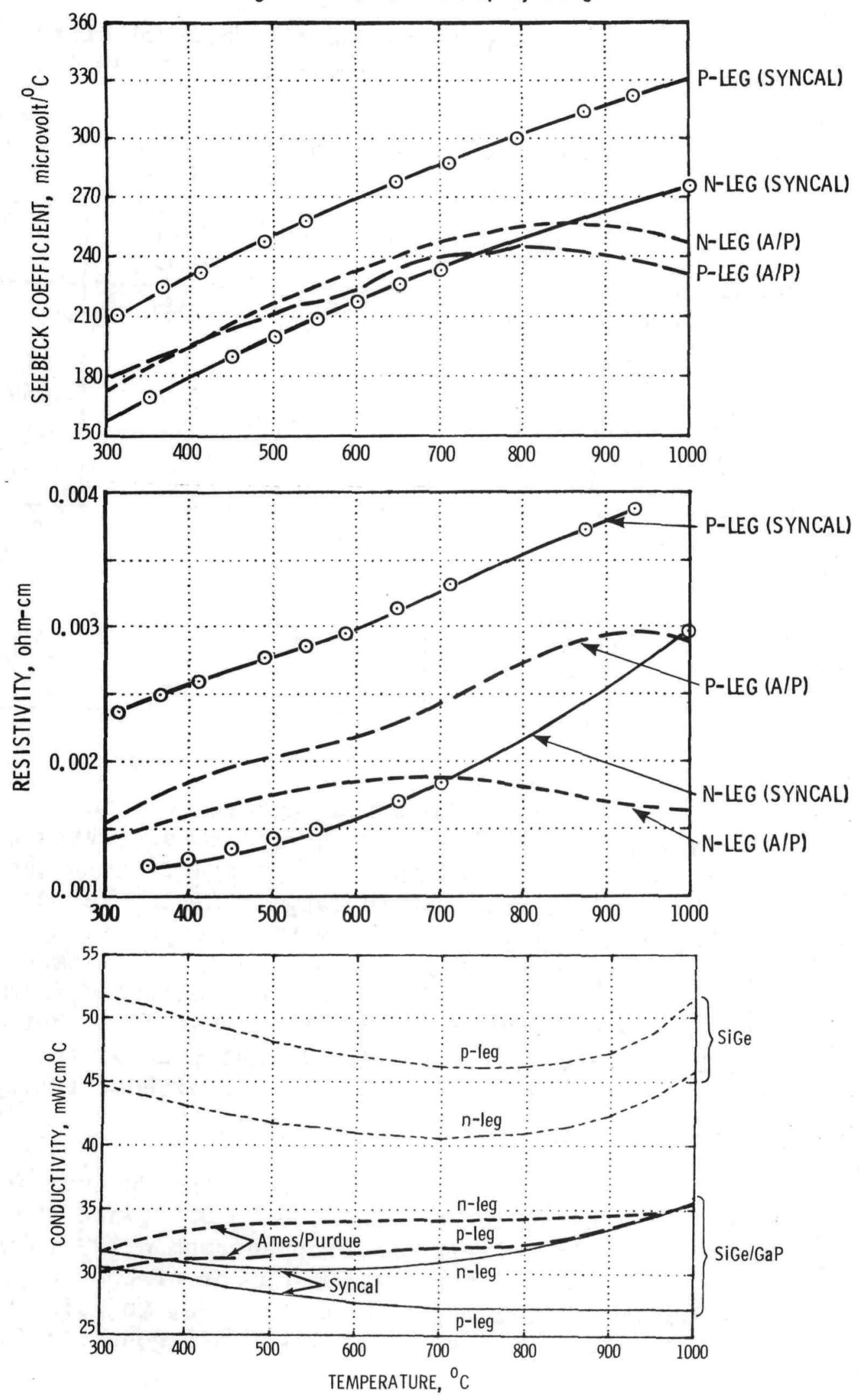
The effect of these changes on the thermoelectric material's performance is summarized in Table 1.

Table 1. Thermoelectric Property Changes and Their Effects

\begin{tabular}{|c|c|c|c|}
\hline & $\begin{array}{c}\text { Syncal } \\
1981\end{array}$ & $\begin{array}{c}\text { Ames/Purdue } \\
1983\end{array}$ & \\
\hline Seebeck Coefficient, $\bar{\alpha}$ & 0.497 & 0.452 & $\mathrm{mV} /{ }^{\circ} \mathrm{C}$ \\
\hline Electrical Resistivity, $\overline{\boldsymbol{p}}_{n}$ & 1.79 & 1.72 & $\mathrm{~m} \Omega-\mathrm{cm}$ \\
\hline$\overline{\boldsymbol{\rho}}_{p}$ & 3.18 & 2.35 & $\mathrm{~m} \Omega-\mathrm{cm}$ \\
\hline Thermal Conductivity, $\overline{\boldsymbol{\kappa}}_{n}$ & 27.8 & 33.9 & $\mathrm{mw} / \mathrm{cm}^{\circ} \mathrm{C}$ \\
\hline$\overline{\boldsymbol{x}}_{p}$ & 31.8 & 32.0 & $\mathrm{mw} / \mathrm{cm}^{\circ} \mathrm{C}$ \\
\hline Figure of Merit, $\bar{z}$ & 0.846 & 0.770 & $10^{-3} K^{-1}$ \\
\hline Opt. Area Ratio, $A_{n} / A_{p}$ & 0.80 & 0.83 & - \\
\hline Opt. Couple Voltage, $V_{O}$ & 0.199 & 0.178 & volt \\
\hline Max, Efficiency, $\quad \boldsymbol{\eta}$ & 10.31 & 9.63 & $\%$ \\
\hline
\end{tabular}

The table shows the Seebeck coefficient, electrical resistivity, and thermal conductivity of the $\mathrm{n}$ - and p-legs, appropriately averaged over the $300^{\circ} \mathrm{C}$-to $-1000^{\circ} \mathrm{C}$ temperature range. As can be seen, the material's figure of merit has dropped from $0.846 \times 10^{-3}$ to $0.770 \times 10^{-3} \mathrm{~K}^{-1}$, and the maximum (optimized) material efficiency has decreased from 10.31 to $9.63 \%$. This is an appreciable drop, but the $9.63 \%$ value is still significantly above the material efficiency of $8.3 \%$ for SiGe without the GaP additive.

The table also shows that the optimum leg area ratio has changed from 0.80 to 0.83 , and the optimum couple voltage has dropped from 0.199 to 0.178 volt. This raises the optimum number of couples for the 3.5-volt multicouple from 18 to 20 , to maintain the desired output voltage of 28 volts per generator slice. 


\subsection{Thermopile Redesign}

Since the optimum number of legs per multicouple has changed from 36 to 40 , the basic leg arrangement and hot-shoe layout described in [1] had to be redesigned. The previous 36 legs had been arranged in a $6 \times 6$ array. For the new, 40-1eg multicouple, a $4 \times 10$ arrangement using rectangular instead of square thermoelectric legs was recommended by Fairchild and adopted by Thermo Electron.

As will be shown, this new arrangement makes possible a number of important advantages, including a much lower hot-shoe resistance, and a significantly improved thermopile fabrication procedure, to provide better hot-shoe alignment with the thermoelectric legs and more reliable glass insulation between adjacent hot shoes.

The old and new leg and hot-shoe arrangements are compared in Figure 10. As can be seen, the hotshoe connectors (heavy rectangles) have a length-towidth ratio of 2.0 in the old $6 \times 6$ array, and only 0.8 in the new $4 \times 10$ array. Therefore, for an equal thickness, the resistance of the hot shoes is reduced by a factor of 2.5 in the new design. There is also a similar reduction in cold-shoe resistance.

Figure 10. Arrangement of Legs (Light Lines) and Hot Shoes (Heavy Lines)

$6 \times 6 \quad(1981)$

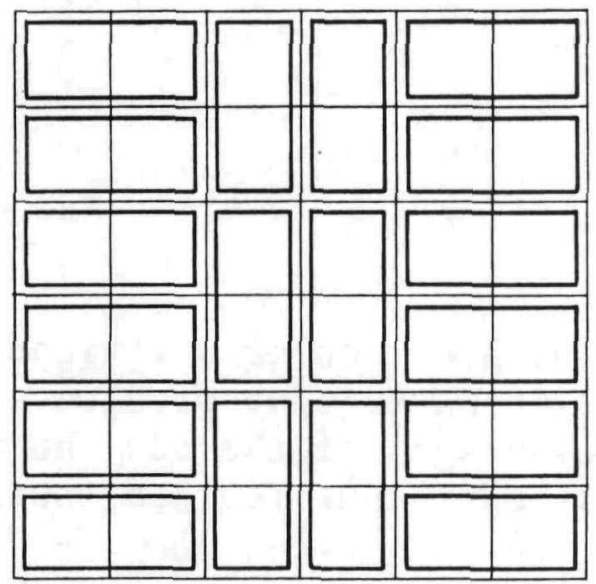

$4 \times 10 \quad(1983)$

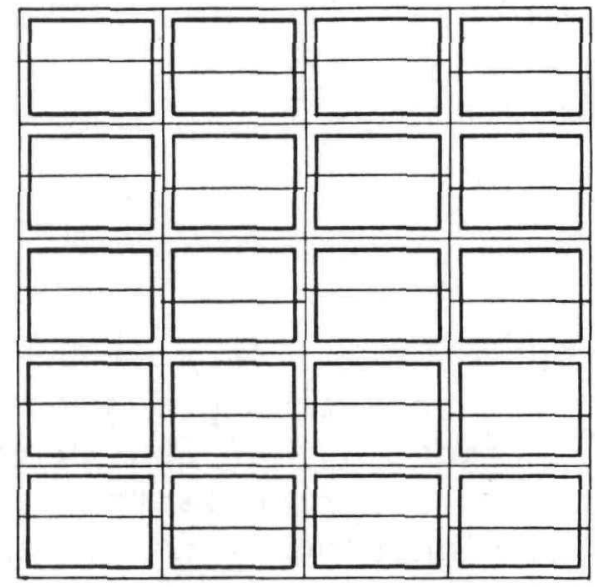


Another important difference illustrated by Figure 10 is the much greater regularity of the hot shoes in the $4 \times 10$ array. As shown in Figure 11, all hot shoes are identical in shape and orientation, which makes it easier to ensure alignment with the underlying SiGe legs during bonding. This is important, because during fabrication of the previous multicouples there had been a tendency for the SiMo hot shoes to shift position during the pressure-loaded bonding operation.

Figure 11. Revised Leg and Hot-Shoe Arrangement

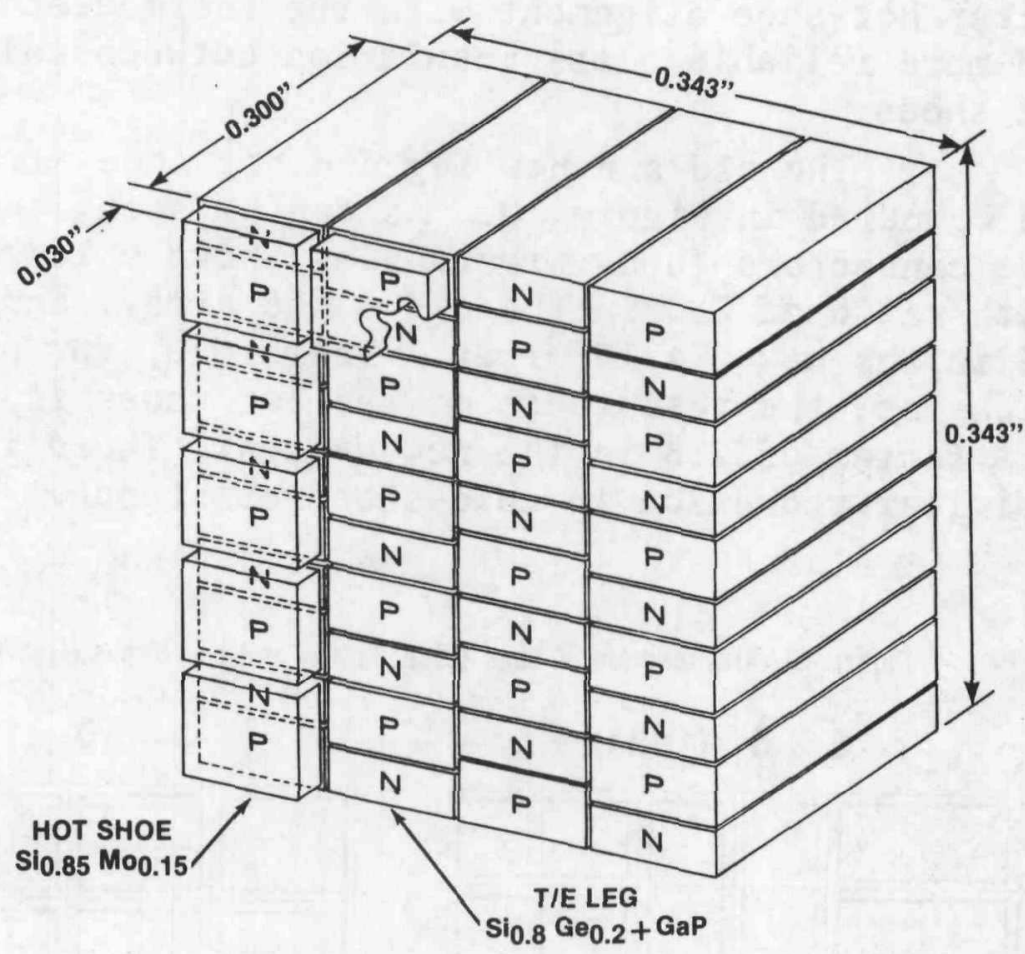

As will be shown, adoption of a revised fabrication process has eliminated that problem. In fact, one of the chief advantages of the new thermopile design is that it has made that revised fabrication process possible. 


\subsection{Revised Fabrication Process}

Post-test examination of the initial multicouples after several hundred hours at design temperature showed no significant degradation in the 2-mil glass insulation between the ȘiGe legs, but did reveal some large voids and resistance degradation in the glass between the SiMo hot shoes. This was attributed to deficiencies in the fabrication process for filling the 6-mil gaps between hot shoes with glass. The process used had apparently been adequate for the original 8-mil hot shoe thickness. But when that thickness had been increased to $30 \mathrm{mils}$ to reduce the hotshoe resistance, the deeper gaps depicted in Figure 11 were not completely filled, leaving unacceptable cavities in the glass. Germanium vapor transport in those cavities accounted for the observed resistance degradation between adjacent hot shoes.

To avoid this problem, a new fabrication process assuring a complete and void-free glass fill was needed. Fortunately, the new $4 \times 10 \mathrm{leg}$ and hotshoe arrangement proposed by Fairchild made possible a much better and simpler fabrication process. As shown in Figure 10, the new arrangement is designed to produce a multicouple thermopile consisting of 20 couples arranged in a $4 \times 5$ array. In each couple, the $\mathrm{n}$ - and $\mathrm{p}$-legs have the optimum area ratio of 0.83 (unlike the earlier $6 \times 6$ multicouples, which used uniform-area legs). Each individual couple (the two legs and the hot shoe) is bonded to its neighbors by a uniform 2-mil glass layer. The new thermopile fabrication process, devised and implemented by Thermo Electron, differs from the previously used fabrication process in that the fabrication, bonding, and glass-coating of the SiMo hot shoes is performed simultaneously with the formation of the SiGe leg assembly. This produces a uniform 2-mil glass layer both between the SiGe legs and between the SiMo hot shoes. The principal steps of the process are illustrated in Figure 12. 
As shown, the process starts with the fabrication of $\mathrm{n}$ - and p-doped SiGe/GaP blocks 0.300" deep, from which slabs with respective thicknesses of 0.0289 " and $0.0348^{\prime \prime}$ are sliced (1). Two such slices are bonded together with a 0.002" glass layer, as before (2); and one edge of the resultant sandwich is diffusion-bonded with germanium to a $0.030 "$ "-thick SiMo hot-shoe strip (3). Five such sandwiches are then stacked up and bonded together by 0.002 " glass layers, as before (4).

The resultant assembly is cut into 0.0826 " slices (5); and four such slices, in alternating orientation, are again bonded together by 0.002 " glass (6). Finally, all surfaces except the cold face of the thermopile block are coated with a 0.003 " glass layer. Note that with the above process, the glass insulation between the hot shoes is applied at the same time (and in the same thickness) as that between the SiGe legs, using a method that had proven successful in previous multicouples.

As mentioned in Reference [4], the thermopile is then glass-bonded to the graphite heat collector, whose mating surface has previously been CVD-coated with a $0.004^{\prime \prime}$ tungsten layer (7). The glass bond, which uses a high-melting $\left(1100^{\circ} \mathrm{C}\right)$ glass, must be carried out before application of the tungsten cold shoes, to avoid high-temperature reactions between tungsten and SiGe. The bonded assembly is shown at the top of Figure 12.

At this point, $0.060^{\prime \prime}$-deep holes are drilled into the two center SiGe legs (8). These will later be used for bonding the multicouple terminal leads.

The cold face of the leg assembly is then coated with 0.001 " tungsten by chemical vapor deposition, and the tungsten coating is separated into individual cold shoes by photo-etching, as shown in Figure 13.

The vapor deposition also produces a complete tungsten coating on the SiGe walls of the terminalwire holes. This is important, because it permits the use of some braze alloys which would otherwise react with the SiGe legs. A number of braze alloys and wire materials are under current investigation. 
Figure 12. Revised Fabrication Process
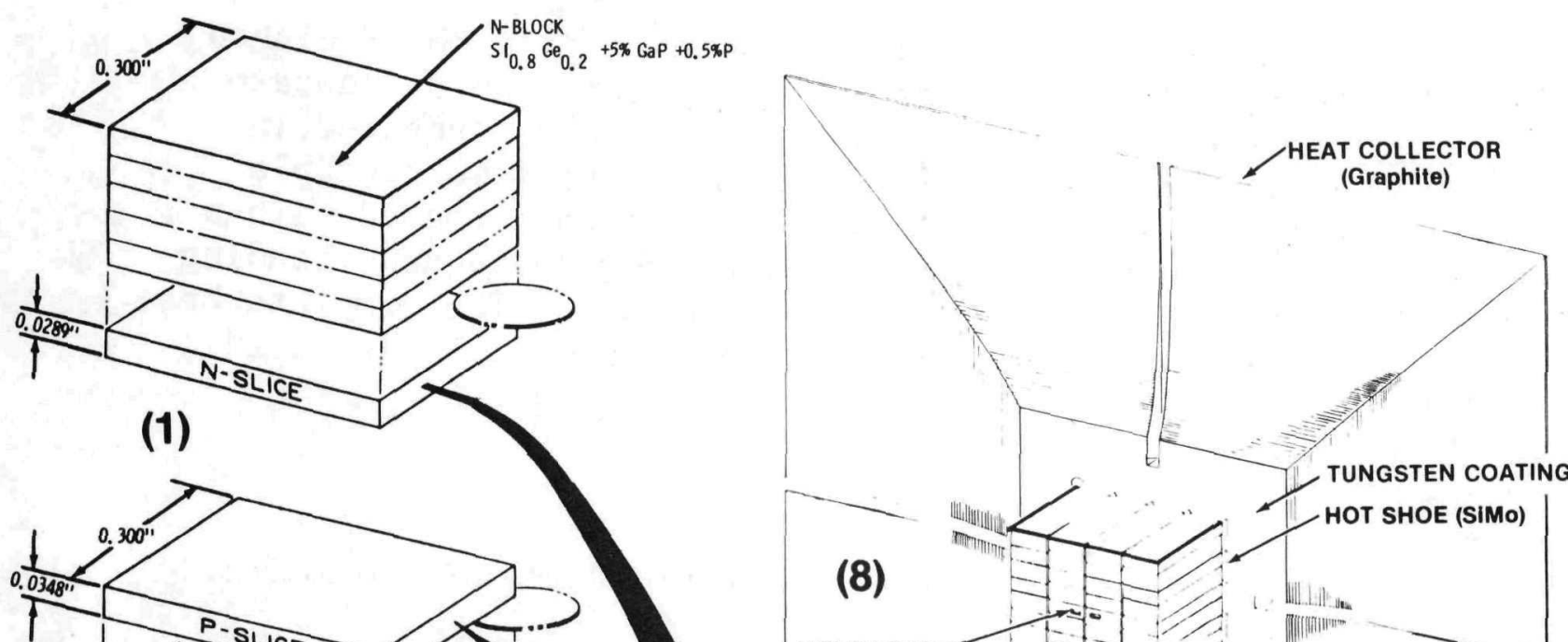

(8)

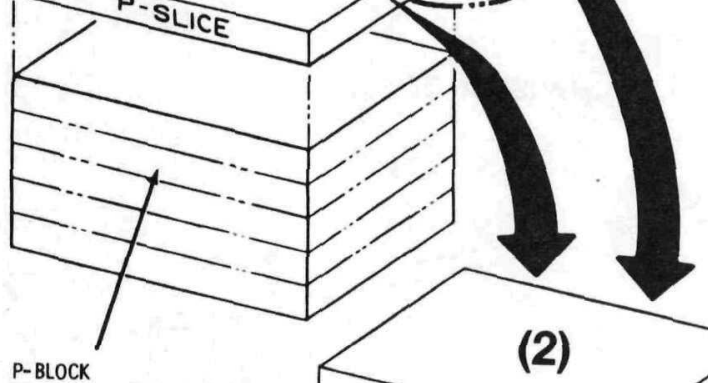

HOLES FOR TERMINAL WIRES

$\mathrm{SI}_{0.8} \mathrm{Ge}_{0.2}+3.5 \% \mathrm{GaP}+0.5 \% \mathrm{~B}$

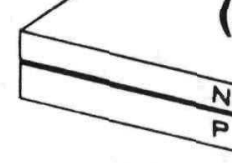

(2)

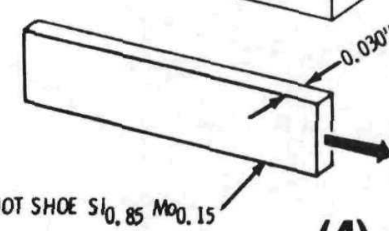

(3)

(4)

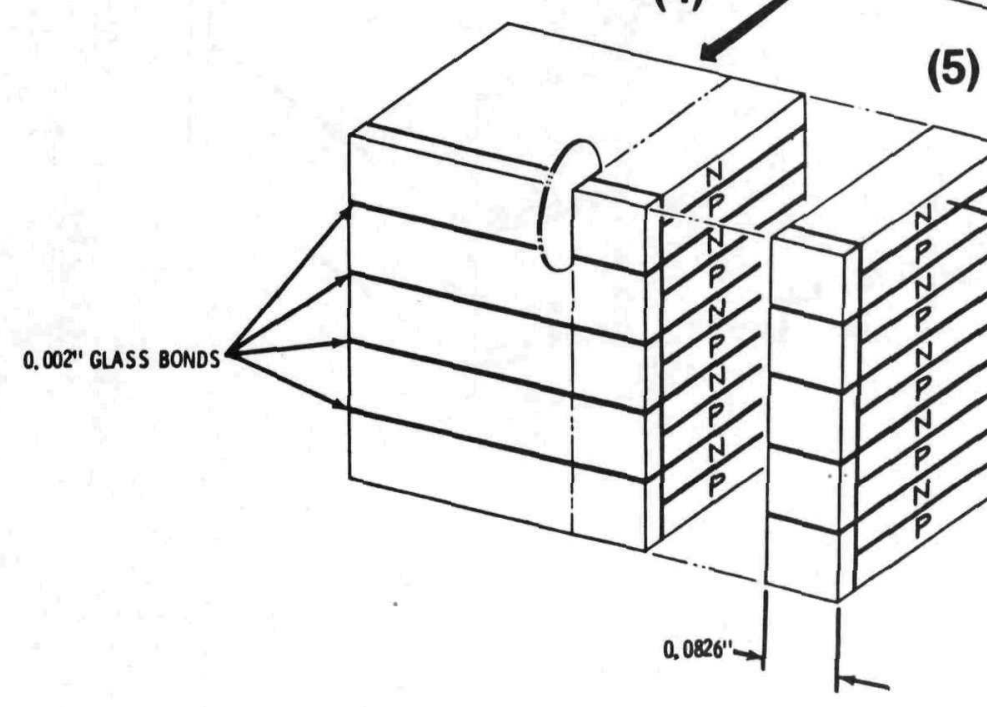

(5)

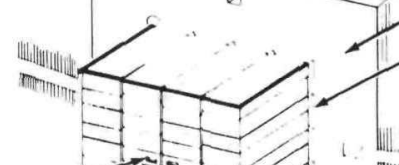
HOT SHOE (SIMO)
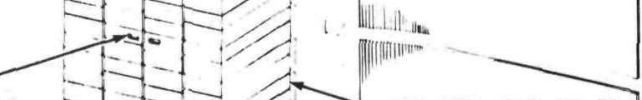
IVINAL WIRES GLASS LAYERS NOT SHOWN

(7)
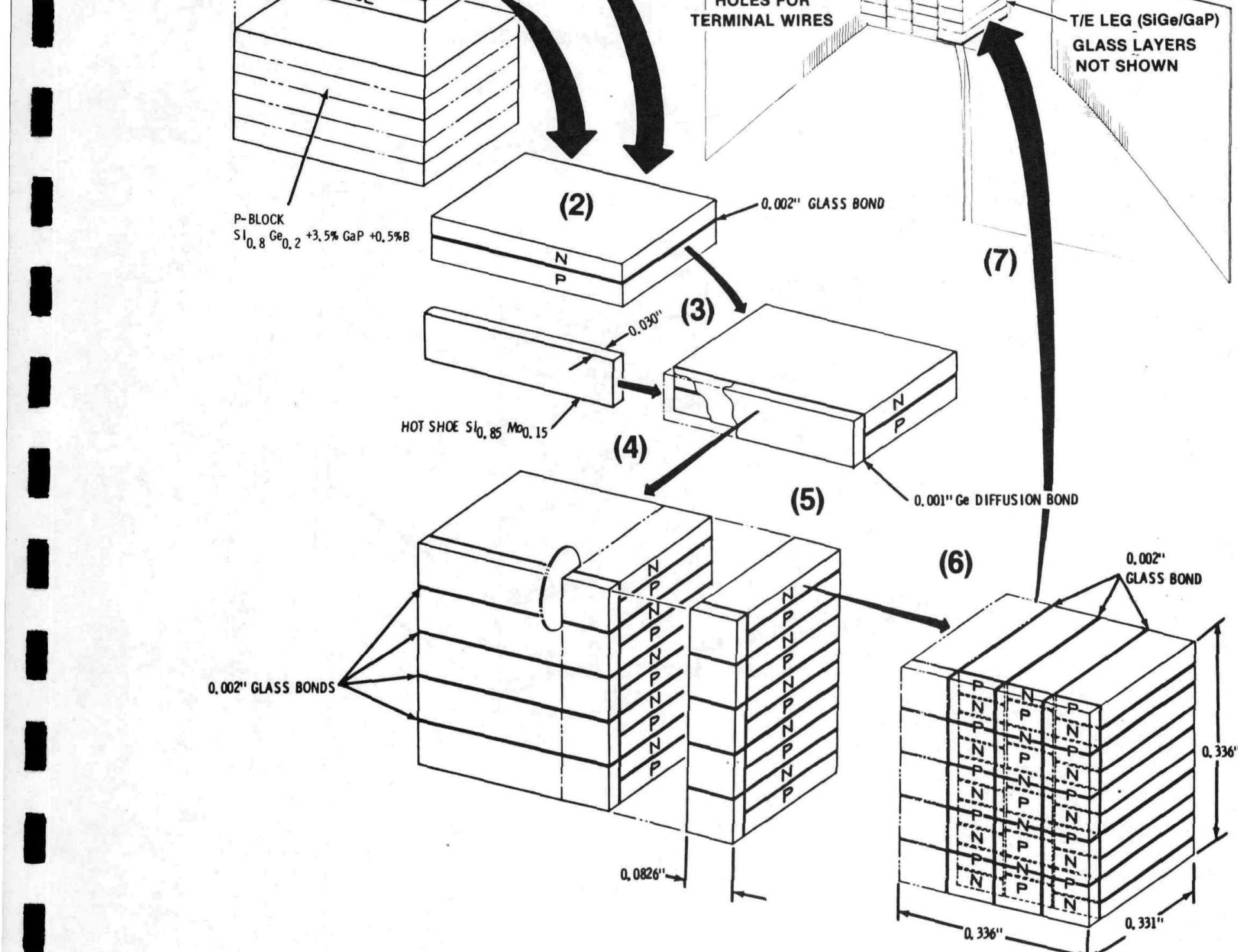

(6)

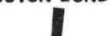


Figure 13 shows a close-up of the finished thermopile's cold face. The numbers shown indicate the sequence in which the 40 legs are connected in series to produce the desired 3.5 volt multicouple output. The exposed cold-face is then coated with a low-temperature $\left(680^{\circ} \mathrm{C}\right)$ glass, for subsequent bonding to the graphite-cushioned cold stud. The complete heat collector/thermopile assembly is shown in Figure 14.

Figure 13. Revised Thermopile Design (Glass Coatings Omitted for Clarity)

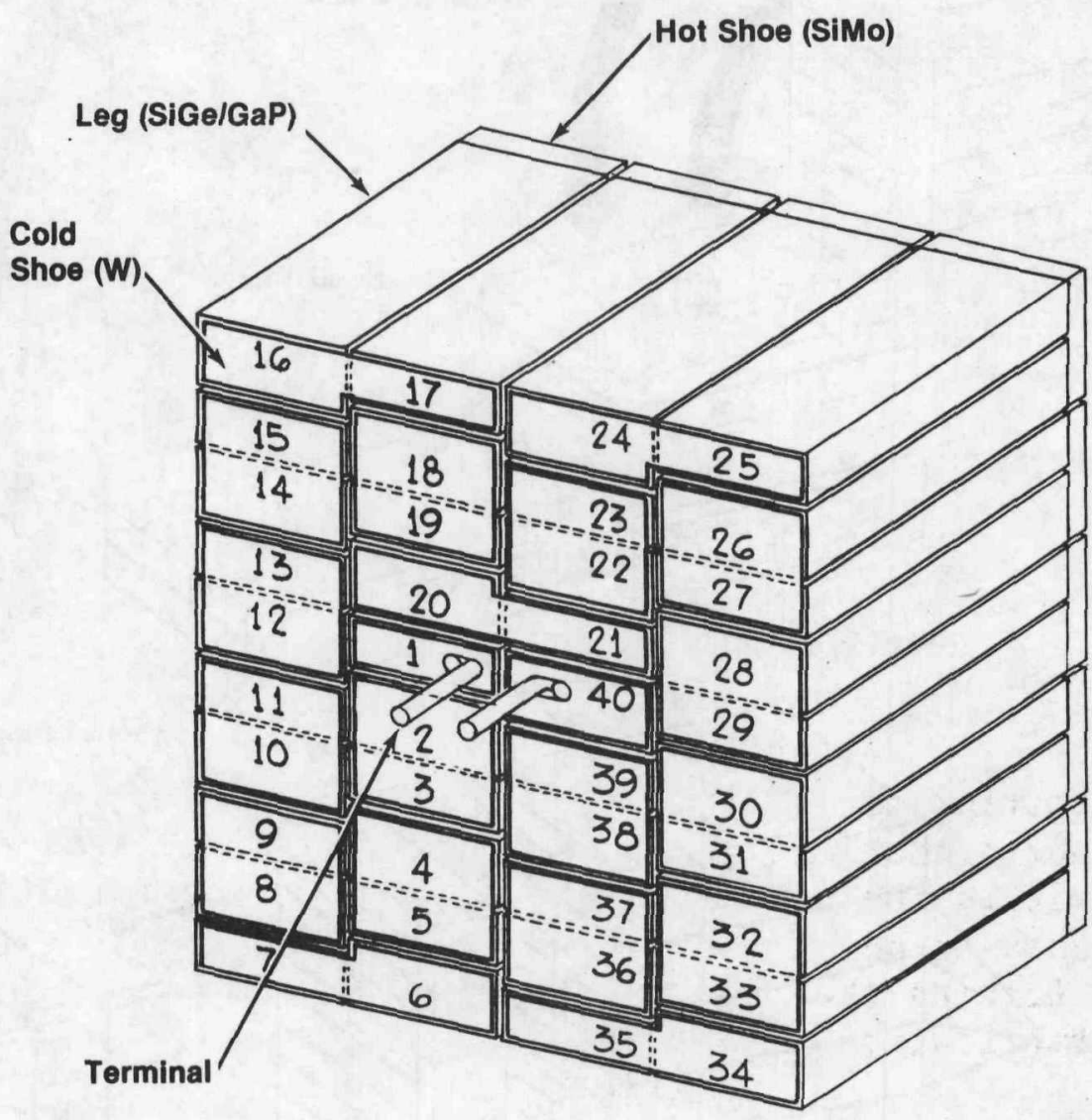


Figure 14. Heat Collector and Thermopile Assembly

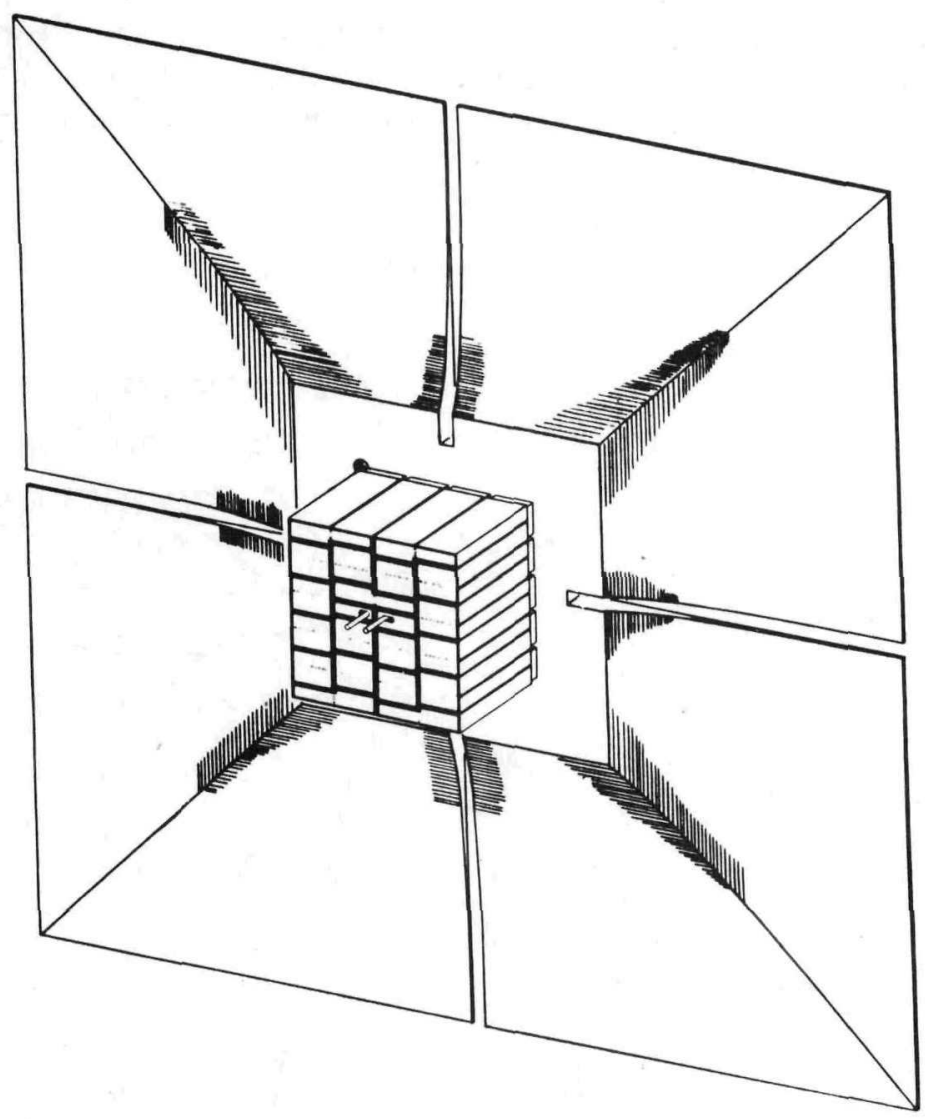

Although the opaque glass coatings have been omitted for clarity in Figures 13 and 14 , it should be noted that all elements of the thermoelectric circuit within the generator are completely encased in glass insulation. This avoids the problem of accidental shorting that had sometimes been encountered during fabrication of previous RTG designs. 
The assembly shown in Figure 14 is then glassbonded to the $0.040 "$-thick graphite cushion, which has previously been Nioro-brazed to the conical-head tungsten cold stud. Figure 15 shows a view of the complete multicouple, split at its midplane. As shown, the alumina-insulated terminal wires pass out through an axial hole in the cold stud, which is subsequently closed off by a glass seal.

Figure 15. Completed Multicouple

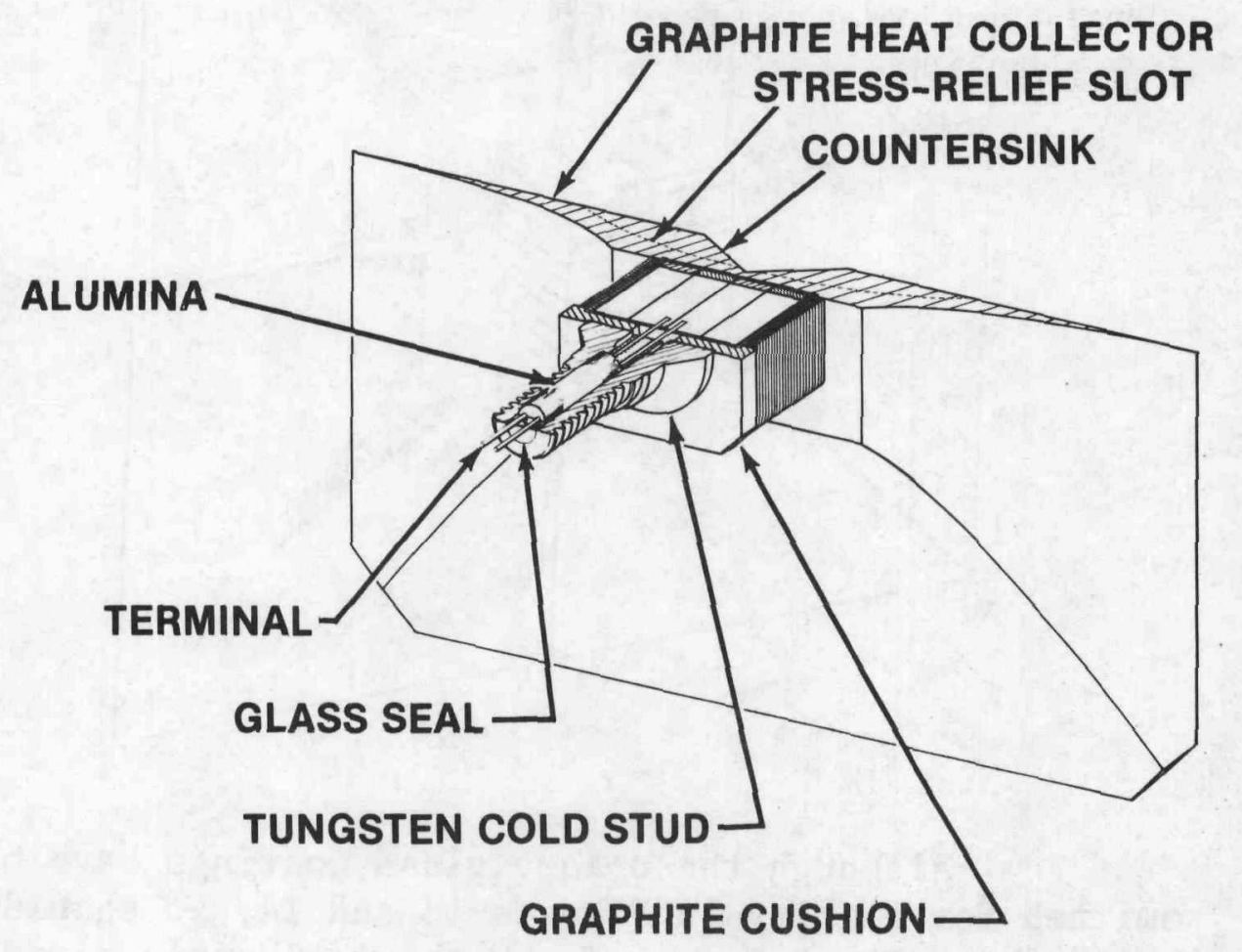

Finally, Figure 16 presents an exploded view of the multicouple and its mounting hardware, and Figure 17 shows the multicouple mounted on the housing wall. (Multifoil insulation has been omitted for clarity.) 
Figure 16. Exploded View of Multicouple and Mounting Hardware

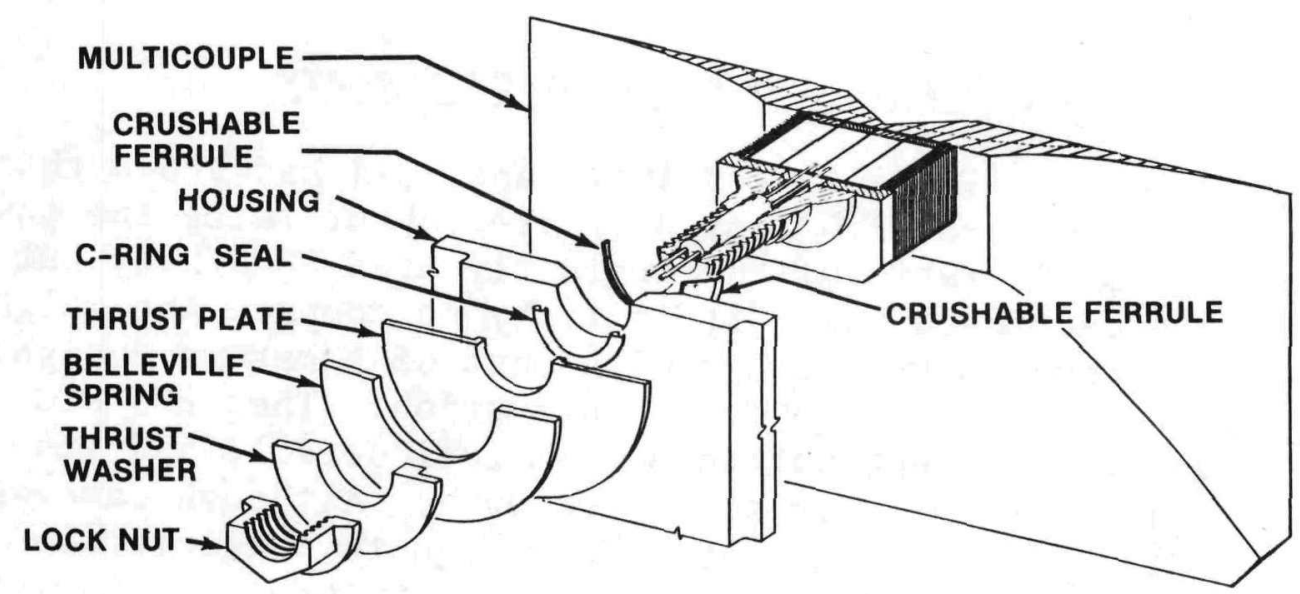

Figure 17. Multicouple Mounted on Housing Wall

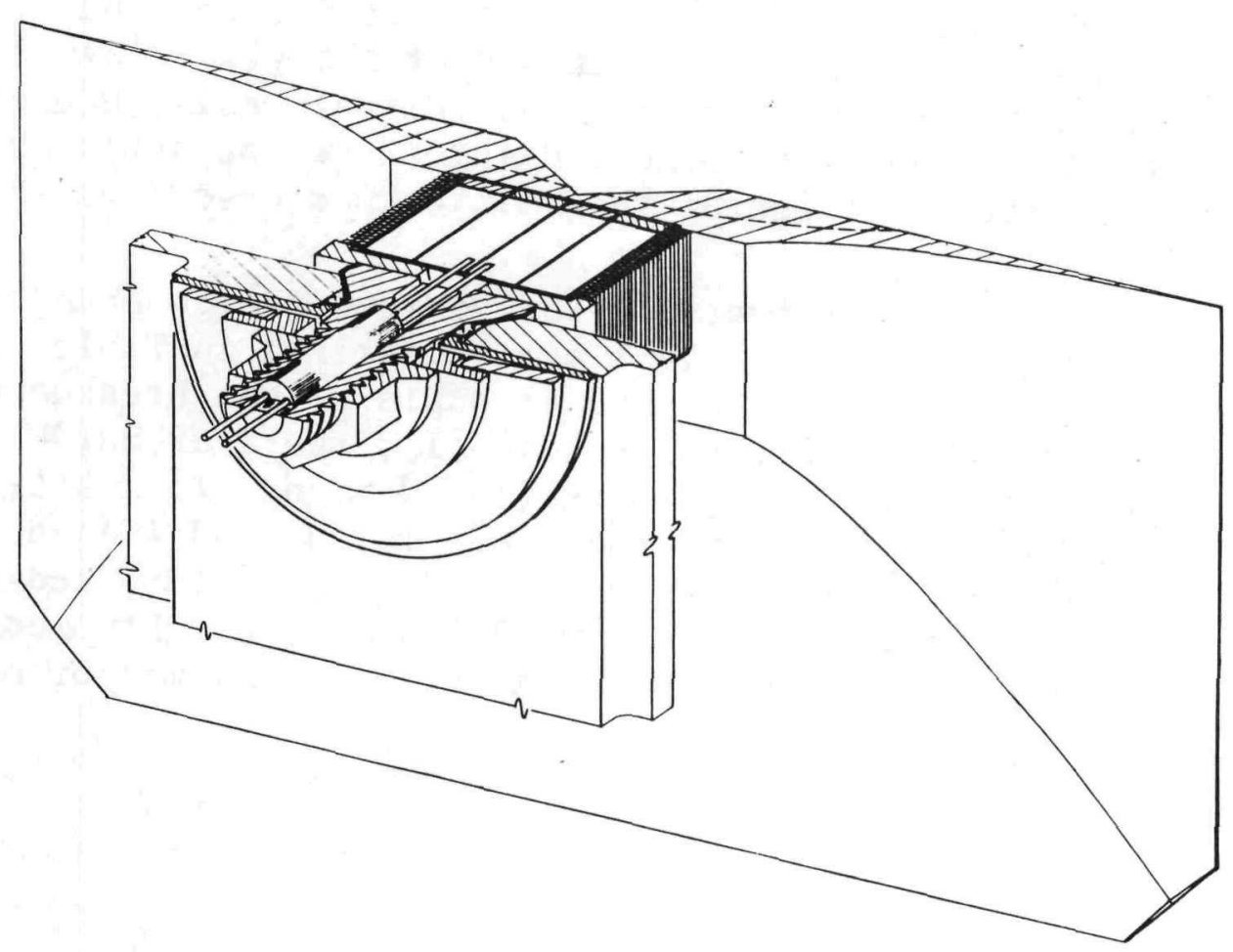




\subsection{Weight and Performance Summary}

The author's 1981 paper [1] had shown that the proposed MITG design offered about twice the powerto-weight ratio of the currently used GPHS/RTG, but had cautioned that "it is risky to compare the weight of a conceptual design with that of a mature design that is actually under construction. The GPHS/RTG design has been worked out in full detail, and its weight is unlikely to change much. Although care was taken in assessing the MITG design to make certain that no parts were overlooked and to prepare realistic weight estimates for all parts, it is of course possible that its weight will grow as the result of unforeseen problems or difficulties during further development."

Three factors have impacted the MITG design weight since 1981: new property measurements of the thermoelectric materials, which have changed significantly; analytical refinements to account more accurately for the electrical resistance of the hot and cold shoes, the thermal conductance of the glass layers and quartz yarn wrap, and the thermal resistance of the cold-end glass bond and interface contact; and the extensive redesign of the multicouple described in Section 2.

The effects of the various design changes on the multicouple's weight are summarized in Table 2 . The table compares the multicouple weight breakdowns of the 1981 conceptual design [1], the 1982 hardware build [2], and the 1983 revised design. The latter two columns include items that were calculated $(C)$ and items that were actually weighed $(W)$, as reported by Thermo Electron. As shown, $95 \%$ of the weight prediction for the revised design is based on actual measurements. 
Table 2. Multicouple Assembly Mass Breakdown (Grams)

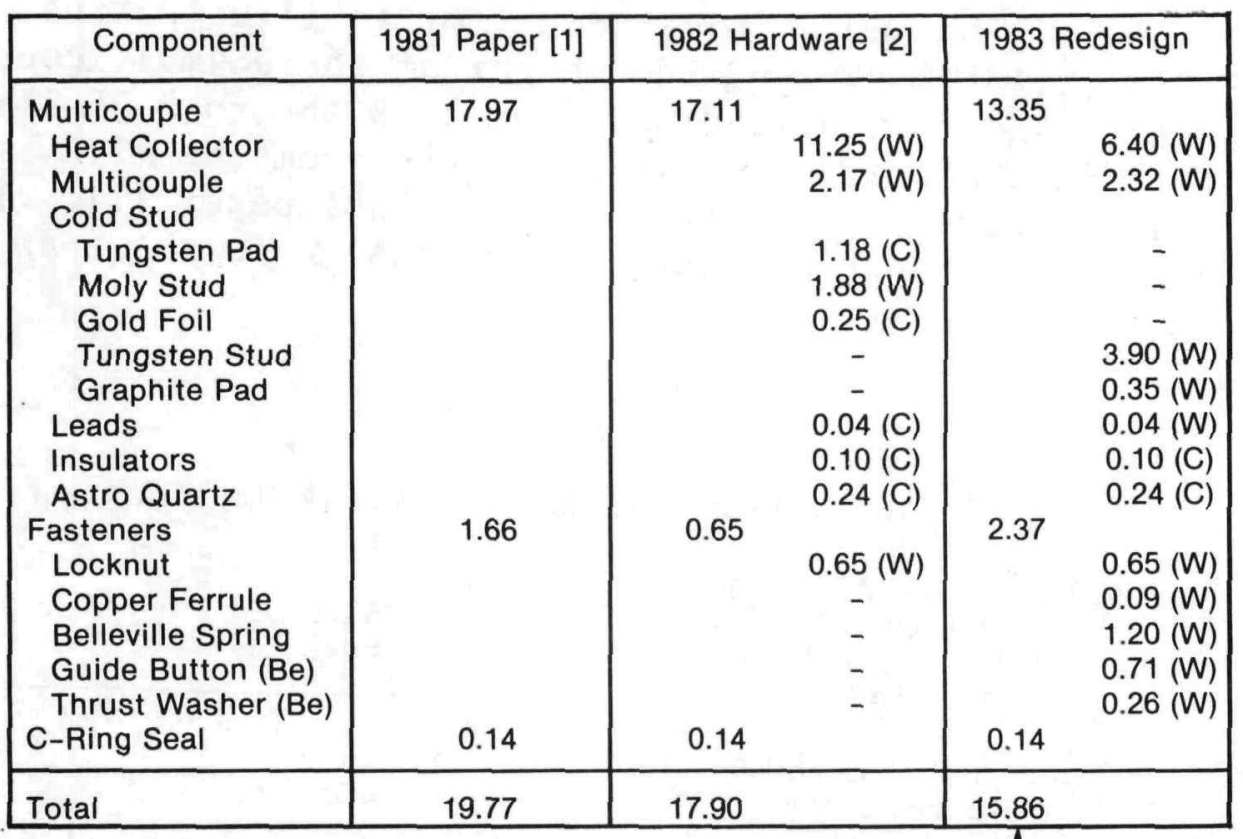

95\% Weighed (W), 5\% Calculated (C)

Table 2 shows that the multicouple built in 1982 actually weighed about $10 \%$ less than the value predicted in the 1981 paper, which is contrary to usual experience. Comparison of the 1983 redesign with the 1982 hardware shows that there were significant weight increases in the fasteners, springs, and cold studs; but that these were more than balanced by the offsetting weight reduction of the graphite heat collector. Thus, the total weight of the 1983 multicouple is even lower than that of the 1982 hardware, for a combined weight reduction of $20 \%$ below the 1981 prediction.

While the above results are reassuring, they have relatively little effect on the power-to-weight ratio of the MITG, since the multicouples represent only a small fraction of the total generator weight. 
As shown in Table 2, for an illustrative 12-slice MITG design, the weight of the 96 multicouples, seals, and fasteners drops from $4.18 \mathrm{lbs}$ to $3.35 \mathrm{lbs}$. This is the only significant change from the MITG weights presented in the author's 1981 paper [1]. The weight breakdown for the revised design is shown in Table 3 .

Table 3. Weight Breakdown for Illustrative 12-Slice MITG

\begin{tabular}{|c|c|c|c|}
\hline $\begin{array}{l}\text { Heat Source Modules (12): } \\
\text { Fuel Pellets (48) } \\
\text { Iridium Capsules (48) } \\
\text { Graphite Parts }\end{array}$ & $\begin{array}{r}15.77 \\
6.19 \\
16.46 \\
\end{array}$ & & \\
\hline & & 38.42 & $(65.1 \%)$ \\
\hline \multicolumn{4}{|l|}{ Heat Source Supports: } \\
\hline Load Spreaders (8) & 0.35 & & \\
\hline PG Buttons (8) & 0.04 & & \\
\hline Bushings ( 8 ) & 0.09 & & \\
\hline Belleville Springs (16) & 0.14 & & \\
\hline Pistons (8) & 0.32 & & \\
\hline Compression Plates (40) & 0.06 & & \\
\hline \multirow[t]{2}{*}{ Preload Screws (8) } & 0.31 & & \\
\hline & & 1.31 & $(2.2 \%)$ \\
\hline \multicolumn{4}{|l|}{ Converter: } \\
\hline Multicouples, Seals, \& Fasteners (96) & 3.35 & & \\
\hline Multifoil Insulation & 2.40 & & \\
\hline Power Connector & 0.35 & & \\
\hline \multirow[t]{2}{*}{ Electrical Circuit } & 0.04 & & \\
\hline & & 6.50 & $(11.0 \%)$ \\
\hline \multicolumn{4}{|l|}{ Generator: } \\
\hline Housing Shell & 6.00 & & \\
\hline Fins (4) & 2.30 & & \\
\hline Auxilliary Coolant Manifold & 0.30 & & \\
\hline Emissive Coating & 0.25 & & \\
\hline End Caps (2) & 1.47 & & \\
\hline C-Seals (2) & 0.06 & & \\
\hline Screws (32) & 0.17 & & \\
\hline Pads, Bushings, \& Nuts & 1.07 & & \\
\hline Gas Management Assembly & 0.29 & & \\
\hline \multirow[t]{2}{*}{ Pressure Release Device } & 0.90 & & \\
\hline & & 12.81 & $(21.7 \%)$ \\
\hline Total & & 59.04 & $(100.0 \%)$ \\
\hline
\end{tabular}


As can be seen, the generator weight consists of $38.4 \mathrm{lbs}$ for the heat source, $1.3 \mathrm{lbs}$ for the heat source support hardware, 6.5 lbs for the converter, and $12.8 \mathrm{lbs}$ for the generator housing, yielding a total of $59.0 \mathrm{lbs}$ for the 12-slice MITG.

The weight change from 59.9 lbs in 1981 to $59.0 \mathrm{lbs}$ in 1983 has relatively little effect on the power-to-weight ratio of the MITG. of much greater importance are the changes in thermoelectric properties and in the electrical and thermal losses discussed below.

The material efficiency of $9.63 \%$ shown earlier in Table 1 reflects the inherent electrical and thermal losses in the thermoelectric material, for an optimized output voltage and leg area ratio. To obtain the corresponding couple efficiency and multicouple efficiency, a number of other electrical and thermal losses must be taken into account.

The couple efficiency is obtained by including the effects of the hot-shoe, cold-shoe, and contact resistances. Since the current seeks a path of least resistance, this computation requires a three-dimensional electrical analysis, to account for current redistribution effects in the SiGe legs and in the hot- and cold-shoes. Using resistivities of $0.85 \mathrm{~m} \Omega-\mathrm{cm}$ for the 0.030 " SiMo hot shoes, $0.038 \mathrm{~m} \Omega-\mathrm{cm}$ for the 0.001 " $\mathrm{W}$ cold shoes, plus $2 \%$ extraneous resistance for the contacts, yielded a couple efficiency of $9.21 \%$.

The multicouple efficiency is obtained by adding the effects of the heat losses through the glass layers and the Astroquartz yarn wrap. Using thermal conductivities of $20.7 \mathrm{mw} / \mathrm{cm}^{\circ} \mathrm{C}$ for the glass and $0.22 \mathrm{mw} / \mathrm{cm}^{\circ} \mathrm{C}$ for the wrap yielded a multicouple efficiency of $8.73 \%$.

Finally, the generator efficiency is obtained by accounting for the heat losses through the multifoil insulation and heat source supports, and the electrical losses in the external circuit. This yielded a generator efficiency of $8.22 \%$. 
Table 4 summarizes the performance parameters for an illustrative 12-slice MITG design. It lists the generator dimensions, operating temperatures, heat balance, and electrical output. Note that the reduced material properties have lowered the generator's power output from 282 watts to 246 watts.

To estimate the weight of smaller or larger generators, it should be noted that the non-modular ends account for 7.0 lbs of the total generator weight, and the 12 modular slices constitute $52.0 \mathrm{lbs}$, or 4.33 lbs per slice. The power output would be essentially proportional to the number of MITG slices; and the total generator height is 2 inches per slice, plus about 6 inches for the two ends (including the heat source preload hardware). 
Table 4. Performance Summary for Illustrative 12-Slice MITG

\begin{tabular}{|c|c|c|}
\hline $\begin{array}{l}\text { Heat Source: } \\
\text { Number of GPHS Modules } \\
\text { Thermal Power per Module (w) } \\
\text { BOL Thermal Power }(w)\end{array}$ & $\begin{array}{r}12 \\
250 \\
3000\end{array}$ & \\
\hline $\begin{array}{l}\text { Thermoelectric Converter: } \\
\text { Number of Multicouples } \\
\text { Number of Couples per Multicouple } \\
\text { P-Leg Thickness (in) } \\
\text { N-Leg Thickness (in) } \\
\text { Leg Width (in) } \\
\text { Module Width and Thickness (in) } \\
\text { Leg Length (in) } \\
\text { Hot-Shoe Thickness (in) } \\
\text { Cold-Shoe Thickness (in) }\end{array}$ & $\begin{array}{r}96 \\
20 \\
0.035 \\
0.029 \\
0.083 \\
0.343 \\
0.300 \\
0.030 \\
0.001\end{array}$ & \\
\hline $\begin{array}{l}\text { Generator Dimensions (in): } \\
\text { Housing Side } \\
\text { Heat Source Height } \\
\text { Generator Height } \\
\text { Generator Span }\end{array}$ & $\begin{array}{r}5.75 \\
25 \\
29 \\
18\end{array}$ & \\
\hline $\begin{array}{l}\text { Temperatures }\left({ }^{\circ} \mathrm{C}\right) \text { : } \\
\text { Heat Source Surface } \\
\text { Hot Junction } \\
\text { Cold Junction } \\
\text { Housing } \\
\text { Fin Root }\end{array}$ & $\begin{array}{r}1100 \\
1000 \\
300 \\
265 \\
260\end{array}$ & \\
\hline $\begin{array}{l}\text { Heat Balance }(w) \text { : } \\
\text { Thermal Power } \\
\text { End Losses } \\
\text { Side Losses } \\
\text { Heat to TE Couples } \\
\text { Heat from TE Couples } \\
\text { Total Heat Rejection }\end{array}$ & $\begin{array}{r}3000 \\
75 \\
100 \\
2825 \\
2578 \\
2753\end{array}$ & \\
\hline $\begin{array}{l}\text { Electrical: } \\
\text { Couples in Series } \\
\text { Strings in Parallel } \\
\text { Module Voltage } \\
\text { Module Current (amp) } \\
\text { Generator Voltage } \\
\text { Generator Current (amp) } \\
\text { Power Output (w) }\end{array}$ & $\begin{array}{r}160 \\
12 \\
3.5 \\
0.734 \\
28 \\
8.81 \\
246.6\end{array}$ & 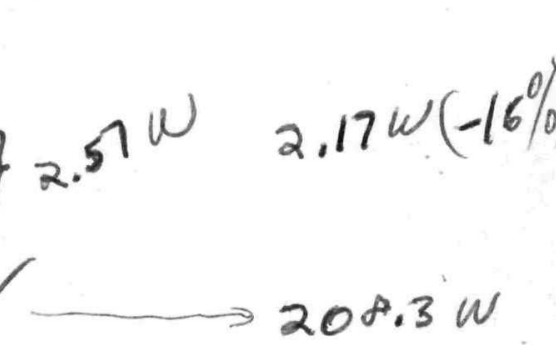 \\
\hline $\begin{array}{l}\text { Efficiency (\%): } \\
\text { Thermal (excl. module losses) } \\
\text { Thermoelectric Material } \\
\text { Couple } \\
\text { Multicouple } \\
\text { Generator }\end{array}$ & $\begin{array}{r}94 \\
9.62 \\
9.21 \\
8.73 \\
8.22\end{array}$ & $7.0 \%$ \\
\hline
\end{tabular}


Finally, Table 5 compares the power-to-weight ratios of the current-generation GPHS/RTG, the 1981 MITG design [1], and the revised MITG design described in this paper. Two options of the latter are presented: The first is for a 12-slice MITG, the same as in 1981 . Because of the reduced material efficiency, this yields only 246 watts. Option 2 is for a 14-slice generator, to yield roughly the same power (288 watts) as the 1981 MITG design and the GPHS/RTG.

Table 5. Specific-Power Comparison (BOL)

\begin{tabular}{|c|c|c|c|c|}
\hline & \multirow{3}{*}{$\begin{array}{c}\text { GPHS/RTG } \\
1983\end{array}$} & \multirow{2}{*}{\multicolumn{3}{|c|}{ MITG }} \\
\hline & & & & \\
\hline & & \multirow{2}{*}{$\frac{1981 \text { Design }}{12}$} & \multicolumn{2}{|c|}{ Revised 1983 Design } \\
\hline Number of Heat Source Modules & 18 & & 12 & 14 \\
\hline Electrical Power, watt & 293 & 282 & 246 & 288 \\
\hline Generator Weight, Ib & 123 & 60 & 59 & 68 \\
\hline $\begin{array}{r}\text { Specific Power, watt/lb } \\
\text { watt } / \mathrm{kg}\end{array}$ & $\begin{array}{l}2.39 \\
5.26\end{array}$ & $\begin{array}{r}4.71 \\
10.37\end{array}$ & $\begin{array}{l}4.18 \\
9.21\end{array}$ & $\begin{array}{l}4.25 \\
9.36\end{array}$ \\
\hline
\end{tabular}

The updated design parameters for the illustrative 14-slice MITG design are summarized in Figure 18. As can be seen, the specific power of the revised MITG is about $10 \%$ lower than that predicted in 1981 , but it is still about $78 \%$ higher than that of current-generation RTGs. This conclusion provides encouragement for the continuing MITG development program. 
Figure 18. Updated MITG Design Parameters

Illustrative Example: 14 Slices, 288 Watts, 68 Lbs, 4.25 W/Lb

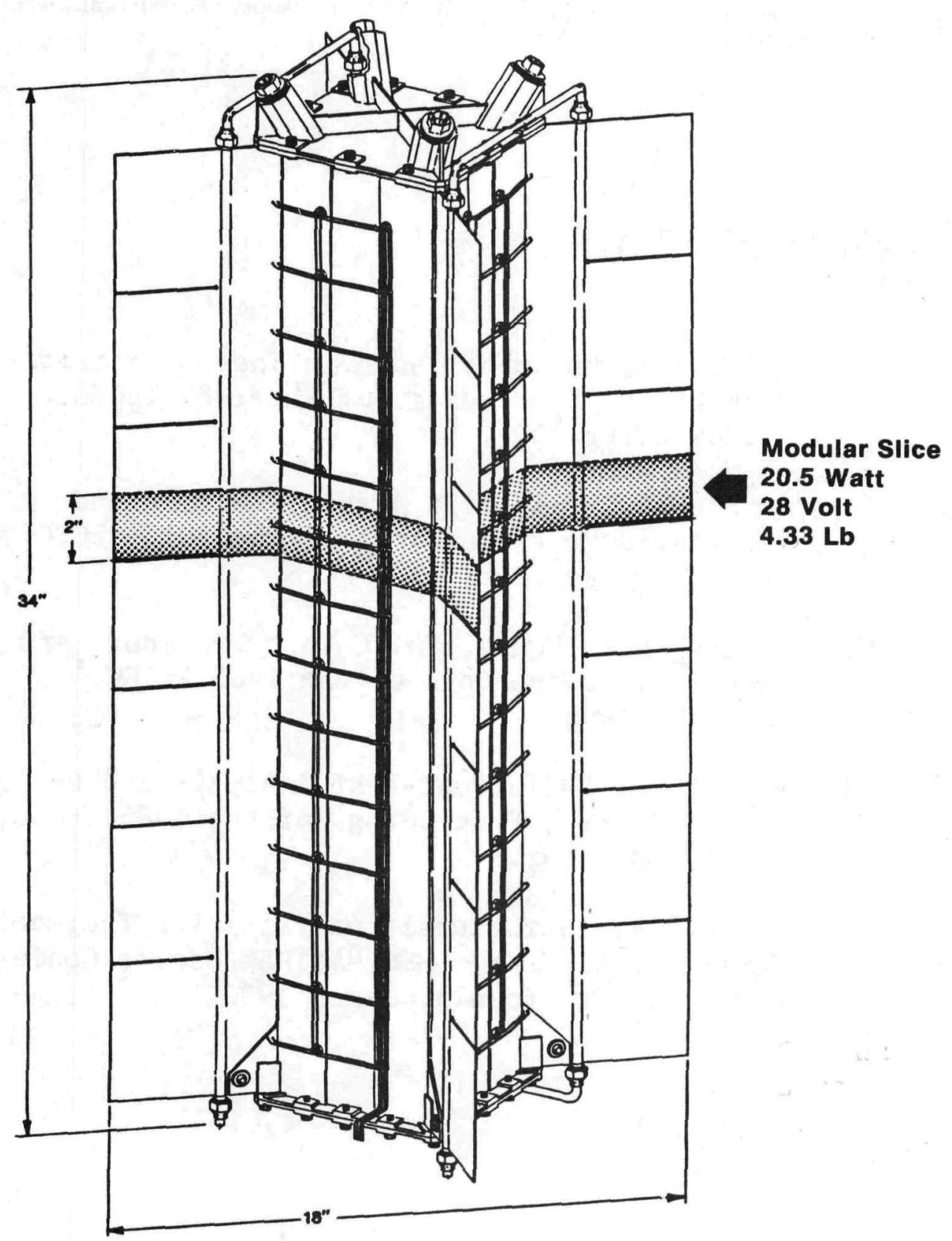




\section{Acknowledgment}

The author takes pleasure in acknowledging that the work described here reflects the contributions of many individuals, including (alphabetically): M. Eck, T. Kline, M. Mukunda, J. Newell, and E. Skrabek of Fairchild; and L. Carrol1, K. Hagen, F. Huffman, and v. Raag of Thermo Electron.

\section{References: (Part E):}

\section{Part:}

A. [1] A. Schock, "Modular Isotopic Thermoelectric Generator", Proceedings of the 1981 IECEC, Volume 1, Pg. 327.

B. [2] A. Schock, "MITG Test Assembly Design and Fabrication", Proceedings of the 1983 IECEC, previous paper.

C. [3] M. Eck, M. Mukunda, "MITG Test Procedure and Results", Proceedings of the 1983 IECEC, previous paper.

D. [4] A. Schock, "MITG Post-Test Analysis and Design Improvements", Proceedings of the 1983 IECEC, preceding paper.

[5] J. Newe11, "Structural Analysis of a Thermoelectric Generator Element", MSC/NASTRAN User's Conference Pasadena, California, March 1983. 